\title{
Stereoselective Palladium-Catalyzed Carbocyclization of Allenic Allylic Carboxylates
}

Johan Franzén, Joakim Löfstedt, Jennica Falk and Jan-E. Bäckvall*.

Department of Organic Chemistry, Arrhenius Laboratory, Stockholm University, SE - 10691

Stockholm, Sweden

\section{Supporting Information}




\section{Preparation of substrates for cyclization.}

\section{Scheme S1}

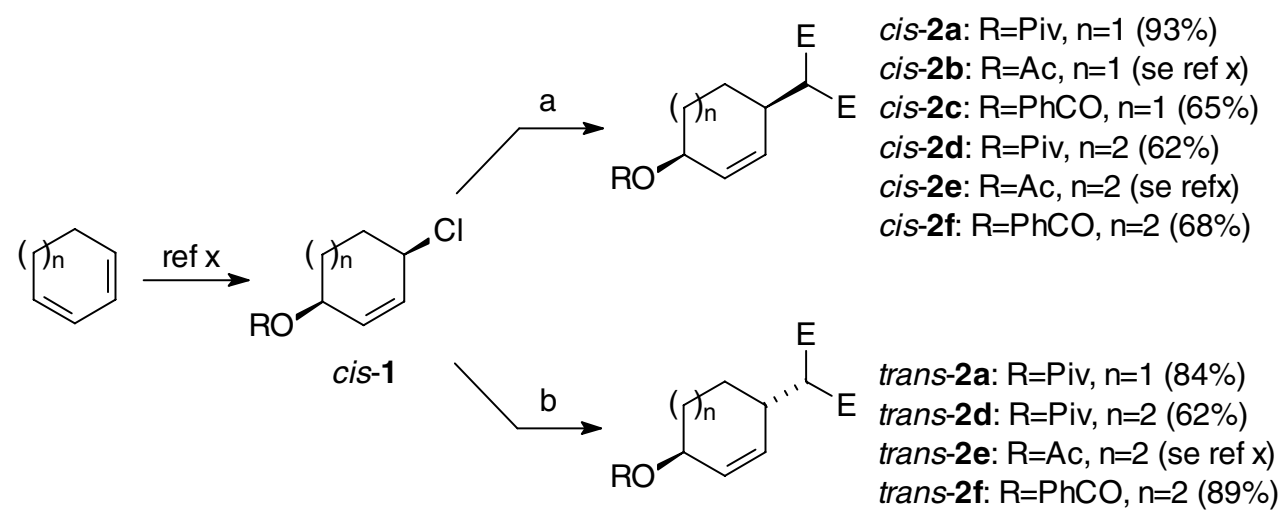

(a) $\mathrm{NaH},\left(\mathrm{CH}_{3} \mathrm{O}_{2} \mathrm{C}\right)_{2} \mathrm{CH}_{2}, \mathrm{Pd}(\mathrm{OAc})_{2}, \mathrm{PPh}_{3}, \mathrm{THF}, \mathrm{rt}$; (b) $\mathrm{NaH},\left(\mathrm{CH}_{3} \mathrm{O}_{2} \mathrm{C}\right)_{2} \mathrm{CH}_{2}, \mathrm{CH}_{3} \mathrm{CN}$, reflux.

\section{Scheme S2}

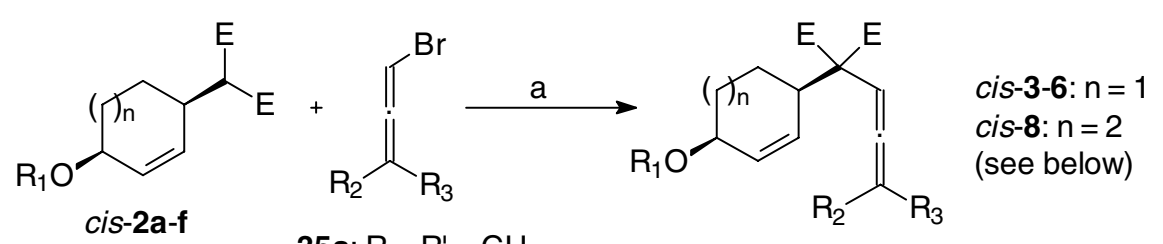

35a: $\mathrm{R}=\mathrm{R}^{\prime}=\mathrm{CH}_{3}$

35b: $R=R^{\prime}=-\left(\mathrm{CH}_{2}\right)_{4}-$

35c: $\mathrm{R}=\mathrm{CH}_{3}, \mathrm{R}^{\prime}=\mathrm{CH}_{2} \mathrm{CH}_{3}$

35d: $\mathrm{R}=\mathrm{CH}_{3}, \mathrm{R}^{\prime}=\left(\mathrm{CH}_{2}\right)_{2} \mathrm{CHCH}_{2}$<smiles>[R9]OC1C=CC(C(F)F)CC1</smiles><smiles>[R]C([R])=C=CBr</smiles>

35a: $\mathrm{R}=\mathrm{R}^{\prime}=\mathrm{CH}_{3}$<smiles>[R]C([R])=C=CC(F)(F)[C]1C=CC(O)CC1</smiles>

trans-3-6: $\mathrm{n}=1$ trans-8: $\mathrm{n}=2$

(see below)

35b: $\mathrm{R}=\mathrm{R}^{\prime}=-\left(\mathrm{CH}_{2}\right)_{4}-$

35c: $\mathrm{R}=\mathrm{CH}_{3}, \mathrm{R}^{\prime}=\mathrm{CH}_{2} \mathrm{CH}_{3}$

35d: $\mathrm{R}=\mathrm{CH}_{3}, \mathrm{R}^{\prime}=\left(\mathrm{CH}_{2}\right)_{2} \mathrm{CHCH}_{2}$

(a) $\mathrm{NaH}, \mathrm{THF}$, reflux.

\section{Scheme S3}




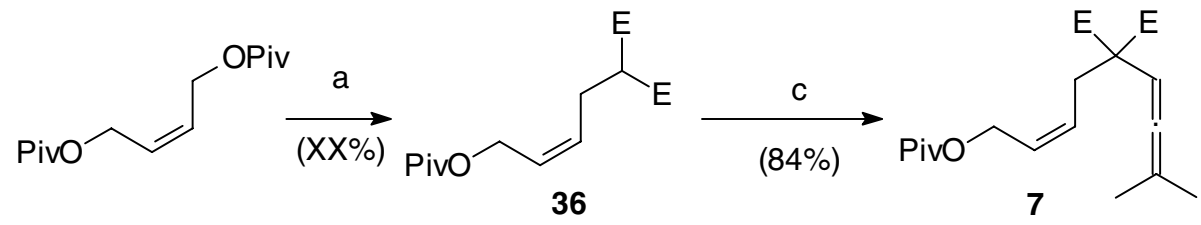

(a) $\mathrm{NaH},\left(\mathrm{CH}_{3} \mathrm{O}_{2} \mathrm{C}\right)_{2} \mathrm{CH}_{2}, \mathrm{Pd}(\mathrm{OAc})_{2}, \mathrm{PPh}_{3}$, THF,reflux; (b) $\mathrm{NaH}, 35 \mathbf{a}$, THF, reflux.

\section{Scheme S4}

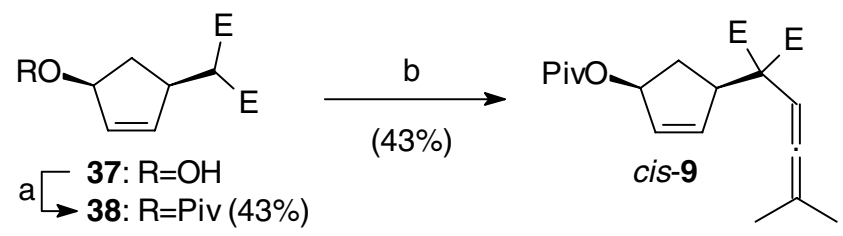

(a) Pivalyl chloride, DMAP, pyridine; (b) NaH, 35a, THF, reflux.

Scheme S5

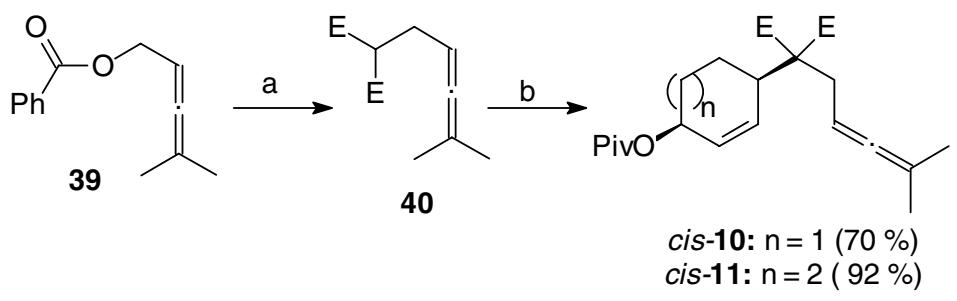

(a) $\mathrm{NaH},\left(\mathrm{CH}_{3} \mathrm{O}_{2} \mathrm{C}\right)_{2} \mathrm{CH}_{2}, \mathrm{Pd}(\mathrm{OAc})_{2}, \mathrm{PPh}_{3}$, THF, reflux; (b) $\mathrm{NaH}$, cis-2a or cis-2d, $\mathrm{Pd}(\mathrm{OAc})_{2}, \mathrm{PPh}_{3}$, THF,rt.

Experimental section ${ }^{1} \mathrm{H}$ NMR (400 or $300 \mathrm{MHz}$ ) and ${ }^{13} \mathrm{C}$ NMR (100 or $\left.75 \mathrm{MHz}\right)$ spectra were recorded using chloroform- $\mathrm{d}_{1}\left(7.26 \mathrm{ppm}{ }^{1} \mathrm{H}, 77 \mathrm{ppm}{ }^{13} \mathrm{C}\right)$ as internal standard. THF was dried over sodium and refluxed under argon atmosphere prior to use.

Chlorocarboxylates cis-1 were prepared from the corresponding dienes according to a previously described procedure. (Bäckvall, J. E.; Granberg, K. L.; Hopkins, R. B. Acta Chem. Scand. 1990, 44, 492.).

Malonates cis-2a, cis-2b, cis-2e, trans-2a and trans-2e were prepared from the corresponding chloroacetates (Franzén, J.; Löfstedt, J.; Dorange, I.; Bäckvall, J. E. J. Am. Chem. Soc. 2002, 124, 11246-11247 and Bäckvall, J. E.; Nyström, J. E.; Nordberg, R. E. J. Am. Chem. Soc. 1985, 107, 3676.).

Bromoallenes 35a-d were prepared from the corresponding disubstituted propargylic alcohols (Sigman, S. S.; Eaton, B. E. J. Am. Chem. Soc. 1996, 118, 11783 and Landor, R. S.; Landor, P. D.; Whiter, P. F. J. Chem. Soc., Perkin Trans. 1 1977, 1710.). 
Hydroxy malonate cis-37 was prepared from 1,3-cyclopentadiene (Gerdil, R.; Liu, H.; Bernardineli, G. Helv. Chim. Acta 1999, 82, 418 and Trost, B. M.; Molander, G. A. J. Am. Chem. Soc. 1981, 103, 5969.)

Allene 39 was prepared from it corresponding alcohol (Löfstedt, J.; Franzén J.; Bäckvall J. E. J. Org. Chem. Soc. 2001, 66, 8015).

General Procedure for the Preparation of Compounds cis-2. Malonate cis-2c. To a solution of dimethyl malonate $(0.58 \mathrm{~mL}, 5.1 \mathrm{mmol})$ in THF $(20 \mathrm{~mL})$ was added $\mathrm{NaH}(60 \%$ in mineral oil, $0.2 \mathrm{~g}, 5.1 \mathrm{mmol})$ and the mixture was stirred for $10 \mathrm{~min}$. To this mixture was added $\mathrm{Pd}(\mathrm{OAc})_{2}$ (0.031 g, $0.14 \mathrm{mmol})$ and $\mathrm{PPh}_{3}(0.12 \mathrm{~g}, 0.46 \mathrm{mmol})$ followed by cis $\mathbf{1}(\mathrm{n}=1, \mathrm{R}=\mathrm{PhCO})$. The resulting mixture was stirred for $2 \mathrm{~h}$ at $\mathrm{rt}$ and partitioned between $\mathrm{Et}_{2} \mathrm{O}(20 \mathrm{ml})$ and $\mathrm{H}_{2} \mathrm{O}(20 \mathrm{ml})$. The aqueous phase was extracted with $\mathrm{Et}_{2} \mathrm{O}(3 \times 10 \mathrm{ml})$ and the combined organic phases were dried $\left(\mathrm{Na}_{2} \mathrm{SO}_{4}\right)$. The solvent was removed in vacuo followed by flash chromatography (pentane:Et $\left.{ }_{2} \mathrm{O}, 90: 10\right)$ to give $1.33 \mathrm{~g}\left(86 \%\right.$ yield) of cis-2c. ${ }^{1} \mathrm{H} \mathrm{NMR}\left(\mathrm{CDCl}_{3}, 400 \mathrm{MHz}\right): \delta 8.02$ (br d, $J=7.5 \mathrm{~Hz}, 2 \mathrm{H}$ ), 7.53 (br t, $J=7.5 \mathrm{~Hz}, 1 \mathrm{H}$ ), 7.42 (br t, $J=7.5 \mathrm{~Hz}, 2 \mathrm{H}$ ), 5.97 (br d, $J=10.2 \mathrm{~Hz}$, $1 \mathrm{H}), 5.89$ (ddd, $J=10.2,2.2,0.9 \mathrm{~Hz}, 1 \mathrm{H}), 5.43(\mathrm{~m}, 1 \mathrm{H}), 3.76(\mathrm{~s}, 6 \mathrm{H}), 3.38(\mathrm{~d}, J=9.1 \mathrm{~Hz}, 1 \mathrm{H})$, 2.00 (ddt, $J=14.2,6.1,3.3 \mathrm{~Hz}, 1 \mathrm{H}$ ), 1.89 (tt, $J=12.1,3.9 \mathrm{~Hz}, 1 \mathrm{H}), 1.79$ (m, $1 \mathrm{H}), 1.66$ (tdd, $J=$ 12.4, 6.9, $3.3 \mathrm{~Hz}, 1 \mathrm{H}) .{ }^{13} \mathrm{C} \mathrm{NMR}\left(\mathrm{CDCl}_{3}, 100 \mathrm{MHz}\right): \delta$ 168.8, 168.7, 166.2, 133.8, 133.1, 130.7, 129.8 (2C), 128.5 (2C), 127.2, 67.0, 56.3 52.8, 52.7, 35.7, 27.4, 22.6.

Malonate cis-2d was prepared from cis-1 ( $\mathrm{n}=2, \mathrm{R}=\mathrm{Piv})$ and dimethyl malonate as described for cis-2c in $62 \%$ yield. ${ }^{1} \mathrm{H}$ NMR $\left(\mathrm{CDCl}_{3}, 400 \mathrm{MHz}\right): \delta{ }^{1} \mathrm{H} \mathrm{NMR}\left(\mathrm{CDCl}_{3}, 400 \mathrm{MHz}\right): \delta 5.61$ (broad s, 2H), 5.77 (broad d, 1H, $J=10.5 \mathrm{~Hz}), 3.56(\mathrm{~s}, 6 \mathrm{H}), 3.29(\mathrm{~d}, J=7.9 \mathrm{~Hz}, 1 \mathrm{H}), 2.80($ broad t, $J=7.9$ $\mathrm{Hz}, 1 \mathrm{H}), 1.83(\mathrm{~m}, 1 \mathrm{H}), 1.7-1.3(\mathrm{~m}, 4 \mathrm{H}), 1.18(\mathrm{~m}, 1 \mathrm{H}), 1.00(\mathrm{~s}, 9 \mathrm{H}) .{ }^{13} \mathrm{C} \mathrm{NMR}\left(\mathrm{CDCl}_{3}, 100 \mathrm{MHz}\right): \delta$ $177.4,168.6,168.5,134.3,131.2,73.2,56.5,52.3$ (2C), 39.4, 38.4, 31.8, 30.5, 26.9 (3C), 26.0.

Malonate cis-2f was prepared from benzoate cis-1 $(\mathrm{n}=2, \mathrm{R}=\mathrm{PhCO})$ and dimethyl malonate as described for cis-2c in 68\% yield. ${ }^{1} \mathrm{H} \mathrm{NMR}\left(\mathrm{CDCl}_{3}, 400 \mathrm{MHz}\right): \delta 8.03$ (br d, $\left.J=8.3 \mathrm{~Hz}, 2 \mathrm{H}\right), 7.54$ (br t, $J=7.4 \mathrm{~Hz}, 1 \mathrm{H}), 7.41$ (br t, $J=7.4 \mathrm{~Hz}, 2 \mathrm{H}$ ), 5.84 (br d, $J=11.5 \mathrm{~Hz}, 1 \mathrm{H}$ ), 5.70 (ddd, $J=11.5$, 4.4, $1.9 \mathrm{~Hz}, 2 \mathrm{H}), 3.73(\mathrm{~s}, 3 \mathrm{H}), 3.72(\mathrm{~s}, 3 \mathrm{H}), 3.48(\mathrm{~d}, J=7.7 \mathrm{~Hz}, 1 \mathrm{H}), 3.04(\mathrm{~m}, 1 \mathrm{H}), 2.03$ (m, 2 $\mathrm{H}), 1.74(\mathrm{~m}, 3 \mathrm{H}), 1.41(\mathrm{tdd}, J=13.5,11.0,2.7 \mathrm{~Hz}, 1 \mathrm{H}) .{ }^{13} \mathrm{C} \mathrm{NMR}\left(\mathrm{CDCl}_{3}, 100 \mathrm{MHz}\right): \delta 169.0$, 168.9, 165.9, 134.6, 133.1, 131.8, 130.7, 129.8 (2C), 128.5 (2C), 74.7, 56.9, 52.7 (2C), 39.8, 32.4, 30.9, 26.6 .

General Procedure for the Preparation of trans-1,4-carboxy malonates trans-2. Malonate trans-2d. Compound cis-1 ( $\mathrm{n}=2, \mathrm{R}=\mathrm{Piv})(1.50 \mathrm{~g}, 6.9 \mathrm{mmol})$ was added under argon to a solution of sodium dimethyl malonate $(8.3 \mathrm{mmol})$ in dry $\mathrm{CH}_{3} \mathrm{CN}(30 \mathrm{~mL})$ (prepared from dimethyl malonate and $\mathrm{NaH}(60 \%$ in mineral oil) as described in the preparation of malonate cis-2c above, followed by removal of the THF in vacuo and replacing it with $\mathrm{CH}_{3} \mathrm{CN}$ ). The resulting mixture was refluxed for $16 \mathrm{~h}$ and partitioned between $\mathrm{Et}_{2} \mathrm{O}(20 \mathrm{ml})$ and $\mathrm{H}_{2} \mathrm{O}(20 \mathrm{ml})$. The aqueous phase was extracted with $\mathrm{Et}_{2} \mathrm{O}(3 \times 10 \mathrm{ml})$ and the combined organic phases were dried $\left(\mathrm{Na}_{2} \mathrm{SO}_{4}\right)$. The solvent was removed by evaporation followed by flash chromatography (pentane: $\mathrm{Et}_{2} \mathrm{O}, 90: 10$ ) to give $1.81 \mathrm{~g}$ (62\% yield) of trans-2d. ${ }^{1} \mathrm{H} \mathrm{NMR}\left(\mathrm{CDCl}_{3}, 400 \mathrm{MHz}\right): \delta 5.70(\mathrm{~d}, J=2.5 \mathrm{~Hz}, 2 \mathrm{H}), 5.31$ (broad dq, $J=8.6,2.5$ $\mathrm{Hz}, 1 \mathrm{H}), 3.73$ (s, 3H), 3.72 (s, 3H), $3.48(\mathrm{~d}, J=8.6 \mathrm{~Hz}, 1 \mathrm{H}), 3.17$ (broad t, $J=9.0 \mathrm{~Hz}, 1 \mathrm{H}), 1.82(\mathrm{~m}$, $4 \mathrm{H}), 1.68(\mathrm{~m}, 1 \mathrm{H}), 1.57(\mathrm{~m}, 1 \mathrm{H}), 1.20(\mathrm{~s}, 9 \mathrm{H}) .{ }^{13} \mathrm{C} \mathrm{NMR}\left(\mathrm{CDCl}_{3}, 100 \mathrm{MHz}\right): \delta 177.9,169.0,168.9$, $133.7,132.7,71.6,56.3,52.8,52.7$ (2C), 38.5, 31.4, 30.4, 27.3 (3C), 22.8.

Malonate trans-2f was prepared from cis-1 $(\mathrm{n}=2, \mathrm{R}=\mathrm{PhCO})$ and dimethyl malonate as described for trans-2d in $61 \%$ yield. ${ }^{1} \mathrm{H} \mathrm{NMR}\left(\mathrm{CDCl}_{3}, 400 \mathrm{MHz}\right): \delta 8.05(\mathrm{br} \mathrm{d}, J=8.4 \mathrm{~Hz}, 2 \mathrm{H}), 7.55(\mathrm{br} \mathrm{t}, J=$ $7.5 \mathrm{~Hz}, 1 \mathrm{H}$ ), 7.43 (br t, $J=7.5 \mathrm{~Hz}, 2 \mathrm{H}$ ), 5.83 (br ddd, $J=11.9,3.8,1.6 \mathrm{~Hz}, 1 \mathrm{H}$ ), 5.76 (br dd, $J=$ 11.9, 4.3, 1.9 Hz, $1 \mathrm{H}), 5.63(\mathrm{~m}, 1 \mathrm{H}), 3.75(\mathrm{~s}, 3 \mathrm{H}), 3.74(\mathrm{~s}, 3 \mathrm{H}), 3.53(\mathrm{~d}, J=7.7 \mathrm{~Hz}, 1 \mathrm{H}), 3.21(\mathrm{~m}$, 
$1 \mathrm{H}), 1.99(\mathrm{~m}, 2 \mathrm{H}), 1.89(\mathrm{~m}, 2 \mathrm{H}), 1.75(\mathrm{~m}, 1 \mathrm{H}), 1.66$ (td, $J=9.1,4.9 \mathrm{~Hz}, 1 \mathrm{H}) .{ }^{13} \mathrm{C} \mathrm{NMR}\left(\mathrm{CDCl}_{3}\right.$, $100 \mathrm{MHz}): \delta$

Allene cis-3b was prepared from cis-2b and bromoallene 35a as described for cis-3a in $63 \%$ yield with the exception that the reaction mixture was refluxed for $1.5 \mathrm{~h} .{ }^{1} \mathrm{H} \mathrm{NMR}\left(\mathrm{CDCl}_{3}, 400 \mathrm{MHz}\right): \delta$ 6.00 (ddt, $J=10.3,2.0,1.0 \mathrm{~Hz}, 1 \mathrm{H}$ ), 5.80 (dddd, $J=10.3,4.6,2.6,1.5 \mathrm{~Hz}, 1 \mathrm{H}$ ), 5.46 (septet, $J=$ $2.9 \mathrm{~Hz}, 1 \mathrm{H}), 5.13(\mathrm{~m}, 1 \mathrm{H}), 3.73(\mathrm{~s}, 3 \mathrm{H}), 3.71(\mathrm{~s}, 3 \mathrm{H}), 2.95(\mathrm{tdt}, J=8.3,2.8,1.7 \mathrm{~Hz}, 1 \mathrm{H}), 2.01(\mathrm{~s}$, $3 \mathrm{H}), 1.90$ (br. d, $J=14.0 \mathrm{~Hz}, 1 \mathrm{H}), 1.82$ (br d, $J=14.3 \mathrm{~Hz}, 1 \mathrm{H}), 1.66(\mathrm{~d}, J=2.9 \mathrm{~Hz}, 6 \mathrm{H}), 1.58$ (m, $2 \mathrm{H}) .{ }^{13} \mathrm{C} \mathrm{NMR}\left(\mathrm{CDCl}_{3}, 100 \mathrm{MHz}\right): \delta 202.4,170.9,170.4,170.3,134.7,125.5,99.6,87.0,66.1$, $61.8,52.8,52.6,41.3,28.3,21.6,20.2,20.1,20.0$.

Allene cis-3c was prepared from cis-2c and bromoallene 35a as described for cis-3a in $65 \%$ yield with the exception that the reaction mixture was refluxed for $1.5 \mathrm{~h} .{ }^{1} \mathrm{H} \mathrm{NMR}\left(\mathrm{CDCl}_{3}, 400 \mathrm{MHz}\right): \delta$ $8.01(\mathrm{~d} J=7.3 \mathrm{~Hz}, 2 \mathrm{H}), 7.54(\mathrm{t}, J=7.3 \mathrm{~Hz}, 1 \mathrm{H}), 7.42$ (t, $J=7.3 \mathrm{~Hz}, 2 \mathrm{H}), 6.08$ (br. d, $J=10.3 \mathrm{~Hz}$, $1 \mathrm{H}), 5.96$ (dddd, $J=10.3,4.6,2.5,1.3 \mathrm{~Hz}, 1 \mathrm{H}), 5.52$ (septet, $J=2.9 \mathrm{~Hz}, 1 \mathrm{H}), 5.40(\mathrm{~m}, 1 \mathrm{H}), 3.74$ (s, $3 \mathrm{H}$ ), 3.71 (s, $3 \mathrm{H}), 3.02$ (br. t, $J=8.1 \mathrm{~Hz}, 1 \mathrm{H}$ ), 2.06 (br. d, $J=13.7 \mathrm{~Hz}, 1 \mathrm{H}), 1.85$ (m, $1 \mathrm{H}$ ), $1.70(\mathrm{~m}, 2 \mathrm{H}), 1.67(\mathrm{~s}, 3 \mathrm{H}), 1.65(\mathrm{~s}, 3 \mathrm{H}) .{ }^{13} \mathrm{C} \mathrm{NMR}\left(\mathrm{CDCl}_{3}, 100 \mathrm{MHz}\right): \delta 202.3,170.4,170.3$, 166.3, 135.0, 133.0, 130.9, 129.8 (2C), 128.5 (2C), 125.7, 99.7, 87.2, 66.6, 61.9, 52.8, 52.6, 41.5, $28.3,20.3,20.2,20.0$.

Allene trans-3a was prepared from trans-2a and bromoallene 35a as described for cis-3a in $62 \%$ yield. ${ }^{1} \mathrm{H}$ NMR $\left(\mathrm{CDCl}_{3}, 400 \mathrm{MHz}\right): \delta 5.83$ (ddd, $\left.J=10.5,3.8,2.0 \mathrm{~Hz}, 1 \mathrm{H}\right), 5.63$ (dddd, $J=10.5$, 3.9, 2.7, $1.6 \mathrm{~Hz}, 1 \mathrm{H}), 5.42$ (septet, $J=2.9 \mathrm{~Hz}, 1 \mathrm{H}), 5.23(\mathrm{~m}, 1 \mathrm{H}), 3.72(\mathrm{~s}, 3 \mathrm{H}), 3.70(\mathrm{~s}, 3 \mathrm{H}), 3.09$ $(\mathrm{m}, 1 \mathrm{H}), 2.10(\mathrm{~m}, 1 \mathrm{H}), 1.80(\mathrm{~m}, 1 \mathrm{H}), 1.66$ (two d, $J=2,9 \mathrm{~Hz}, 6 \mathrm{H}), 1.58(\mathrm{~m}, 2 \mathrm{H}) 1.17(\mathrm{~s}, 9 \mathrm{H})$. ${ }^{13} \mathrm{C}$ NMR $\left(\mathrm{CDCl}_{3}, 100 \mathrm{MHz}\right): \delta 202.4,178.5,170.4,170.2,131.8,128.8,99.6,86.9,69.6,62.1$, 52.8, 52.7, 41.1, 38.9, 28.4, 27.3 (3C), 23.4, 20.2, 20.0.

Allene cis-4a was prepared from cis-2a and bromoallene $\mathbf{3 5} \mathbf{b}$ as described for cis-3a in $65 \%$ yield. ${ }^{1} \mathrm{H}$ NMR $\left(\mathrm{CDCl}_{3}, 400 \mathrm{MHz}\right): \delta 6.01($ broad d, $J=10.4 \mathrm{~Hz}, 1 \mathrm{H}), 5.81$ (dqt, $J=10.4,3.0,1.5$ $\mathrm{Hz}, 1 \mathrm{H}), 5.50$ (septet, $J=1.8 \mathrm{~Hz}, 1 \mathrm{H}), 5.08(\mathrm{~m}, 1 \mathrm{H}), 3.72(\mathrm{~s}, 3 \mathrm{H}), 3.69$ (s, $3 \mathrm{H}), 2.96$ (m, $1 \mathrm{H})$, $2.07(\mathrm{~m}, 4 \mathrm{H}), 1.85$ (broad d, $J=14.1 \mathrm{~Hz}, 1 \mathrm{H}), 1.70($ broad t, $J=14.1 \mathrm{~Hz}, 1 \mathrm{H}), 1.59(\mathrm{~m}, 4 \mathrm{H}), 1.49$ $(\mathrm{m}, 4 \mathrm{H}), 1.14(\mathrm{~s}, 9 \mathrm{H}) .{ }^{13} \mathrm{C} \mathrm{NMR}\left(\mathrm{CDCl}_{3}, 100 \mathrm{MHz}\right): \delta 198.9,178.1,170.4,170.3,134.6,125.8$, 106.7, 87.0, 65.7, 61.9, 52.8, 52.5, 41.5, 38.9, 31.1, 30.9, 28.1, 27.5 (2C), 27.3 (3C), 26.1, 20.0.

Allene cis-4b was prepared from cis-2b and bromoallene $\mathbf{3 5 b}$ as described for cis-3a in $44 \%$ yield with the exception that the reaction mixture was refluxed for $1.5 \mathrm{~h} .{ }^{1} \mathrm{H} \mathrm{NMR}\left(\mathrm{CDCl}_{3}, 400\right.$ MHz): $\delta 5.99$ (ddt, $J=10.3,2.0,1.0 \mathrm{~Hz}, 1 \mathrm{H}$ ), 5.77 (dddd, $J=10.3,4.6,2.7,1.5 \mathrm{~Hz}, 1 \mathrm{H}$ ), 5.45 (quintet, $J=2.2 \mathrm{~Hz}, 1 \mathrm{H}), 5.10(\mathrm{~m}, 1 \mathrm{H}), 3.71(\mathrm{~s}, 3 \mathrm{H}), 3.69(\mathrm{~s}, 3 \mathrm{H}), 2.92(\mathrm{~m}, 1 \mathrm{H}), 2.02(\mathrm{~m}, 4 \mathrm{H})$, $1.98(\mathrm{~s}, 3 \mathrm{H}), 1.82$ (br d, $J=14.3 \mathrm{~Hz}, 1 \mathrm{H}), 1.71$ (ddt, $J=14.3,12.5,4.2 \mathrm{~Hz}, 1 \mathrm{H}), 1.52(\mathrm{~m}, 8 \mathrm{H}) .{ }^{13} \mathrm{C}$ $\operatorname{NMR}\left(\mathrm{CDCl}_{3}, 100 \mathrm{MHz}\right): \delta 198.9,170.8,170.4,170.3,134.7,125.6,106.6,86.9,66.1,61.9,52.8$, 52.6, 41.4, 31.1, 30.9, 28.2, 27.4, 27.3, 26.1, 21.5, 20.1 .

Allene cis-4c was prepared from cis-2c and bromoallene 35b as described for cis-3a in $70 \%$ yield with the exception that the reaction mixture was refluxed for $1.5 \mathrm{~h} .{ }^{1} \mathrm{H} \mathrm{NMR}\left(\mathrm{CDCl}_{3}, 400 \mathrm{MHz}\right): \delta$ $8.01(\mathrm{~d} J=7.6 \mathrm{~Hz}, 2 \mathrm{H}), 7.53(\mathrm{t}, J=7.6 \mathrm{~Hz}, 1 \mathrm{H}), 7.41(\mathrm{t}, J=7.6 \mathrm{~Hz}, 2 \mathrm{H}), 6.09(\mathrm{dd}, J=10.2,1.8$ $\mathrm{Hz}, 1 \mathrm{H}$ ), 5.94 (dddd, $J=10.2,4.8,2.5,1.3 \mathrm{~Hz}, 1 \mathrm{H}$ ), 5.53 (quintet, $J=2.2 \mathrm{~Hz}, 1 \mathrm{H}$ ), 5.39 (m, $1 \mathrm{H}$ ), $3.73(\mathrm{~s}, 3 \mathrm{H}), 3.70(\mathrm{~s}, 3 \mathrm{H}), 3.03(\mathrm{~m}, 1 \mathrm{H}), 2.07$ (m, $4 \mathrm{H}), 1.84(\mathrm{~m}, 1 \mathrm{H}), 1.69$ (m, $2 \mathrm{H}), 1.62-1.43$ $(\mathrm{m}, 7 \mathrm{H}) .{ }^{13} \mathrm{C} \mathrm{NMR}\left(\mathrm{CDCl}_{3}, 100 \mathrm{MHz}\right): \delta 200.0,170.4,170.3,166.2,135.1,133.0,130.9,129.8$ (2C), 128.5 (2C), 125.7, 106.8, 87.0, 66.7, 61.9, 52.8, 52.6, 41.5, 31.2, 31.0, 28.3, 27.5, 27.3, 26.1, 20.2 . 
Allene cis-5a was prepared as a 1:1 mixture of diastereoisomers from cis-2a and bromoallene 35c as described for cis-3a in 67\% yield. ${ }^{1} \mathrm{H}$ NMR $\left(\mathrm{CDCl}_{3}, 400 \mathrm{MHz}\right): \delta 6.03(\mathrm{~m}, 2 \mathrm{H}), 5.81(\mathrm{~m}, 2 \mathrm{H})$, 5.60 (two overlapping sextets, $J=3.1 \mathrm{~Hz}, 2 \mathrm{H}$ ), $5.09(\mathrm{~m}, 2 \mathrm{H}$ ), 3.73 (two s, tot. $6 \mathrm{H}$ ), 3.70 (two s, tot. $6 \mathrm{H}), 2.94(\mathrm{~m}, 2 \mathrm{H}), 1.98-1.84(\mathrm{~m}, 6 \mathrm{H}), 1.76-1.50(\mathrm{~m}, 12 \mathrm{H}), 1.16(\mathrm{~s}, 18 \mathrm{H}), 0.96$ (two overlapping t, $J=7.3 \mathrm{~Hz}$, tot $6 \mathrm{H}) .{ }^{13} \mathrm{C} \mathrm{NMR}\left(\mathrm{CDCl}_{3}, 100 \mathrm{MHz}\right): \delta 201.3(2 \mathrm{C}), 178.2(2 \mathrm{C}), 170.3$ (4C), 134.7, 134.6, 125.8 (2C), 106.1, 106.0, 89.2, 89.1, 65.7, 65.6, 61.9, 61.8, 52.7 (2C), 52.5 (2C), 41.8, 41.4, 38.9 (2C), 28.1 (2C), 27.3 (6C), 27.1, 27.0, 20.0 (2C), $18.818 .4,12.2(2 \mathrm{C})$.

Allene cis-5b was prepared as a 1:1 mixture of diastereoisomers from cis-2b and bromoallene 35c as described for cis-3a in $42 \%$ yield with the exception that the reaction mixture was refluxed for $1.5 \mathrm{~h}$. ${ }^{1} \mathrm{H} \mathrm{NMR}\left(\mathrm{CDCl}_{3}, 400 \mathrm{MHz}\right): \delta 6.01$ (br d, $\left.J=10.2 \mathrm{~Hz}, 2 \mathrm{H}\right), 5.77$ (br d, $J=10.2, \mathrm{~Hz}, 2$ H), 5.56 (hextet, $J=2.9 \mathrm{~Hz}, 1 \mathrm{H}), 5.53$ (hextet, $J=2.9 \mathrm{~Hz}, 1 \mathrm{H}), 5.10(\mathrm{~m}, 2 \mathrm{H}), 3.70(\mathrm{~s}, 3 \mathrm{H}), 3.69$ $(\mathrm{s}, 3 \mathrm{H}), 3.68(\mathrm{~s}, 3 \mathrm{H}), 3.67(\mathrm{~s}, 3 \mathrm{H}), 2.90(\mathrm{~m}, 2 \mathrm{H}), 1.98(\mathrm{~s}, 6 \mathrm{H}), 1.89(\mathrm{~m}, 6 \mathrm{H}), 1.75-1.47$ (overlapping peaks, including $\mathrm{d}(J=2.9 \mathrm{~Hz})$ at $1.64 \mathrm{ppm}$ and $\mathrm{d}(J=2.9 \mathrm{~Hz})$ at 1.63 , total $12 \mathrm{H}), 0.94$ (t, $J=7.4 \mathrm{~Hz}, 3 \mathrm{H}), 0.93(\mathrm{t}, J=7.4 \mathrm{~Hz}, 3 \mathrm{H}) .{ }^{13} \mathrm{C} \mathrm{NMR}\left(\mathrm{CDCl}_{3}, 100 \mathrm{MHz}\right): \delta 201.3(2 \mathrm{C}), 170.8$ (2C), 170.3 (2C), 170.2 (2C), 134.8, 134.7, 125.6 (2C), 106.0, 105.9, 89.1, 89.0, 66.1 (2C), 61.8, 61.7, 52.7 (2C), 52.5 (2C), 41.5, 41.3, 28.2 (2C), 27.1, 27.0, 21.5 (2C), $20.1(2 \mathrm{C}), 18.8,18.4,12.1$ (2C).

Allene cis-5c was prepared as a 1:1 mixture of diastereoisomers from cis-2c and bromoallene 35c as described for cis-3a in $72 \%$ yield with the exception that the reaction mixture was refluxed for 1.5 h. ${ }^{1} \mathrm{H} \mathrm{NMR}\left(\mathrm{CDCl}_{3}, 400 \mathrm{MHz}\right): \delta 8.00$ (br d, $\left.J=7.3 \mathrm{~Hz}, 4 \mathrm{H}\right), 7.51$ (br t, $\left.J=7.3 \mathrm{~Hz}, 2 \mathrm{H}\right), 7.40$ (br t, $J=7.3 \mathrm{~Hz}, 4 \mathrm{H}), 6.10$ (br d, $J=10.1 \mathrm{~Hz}, 2 \mathrm{H}$ ), 5.98 (br d, $J=10.3$, Hz, $2 \mathrm{H}$ ), 5.63 (hextet, $J=$ $2.9 \mathrm{~Hz}, 1 \mathrm{H}), 5.60$ (hextet, $J=2.9 \mathrm{~Hz}, 1 \mathrm{H}), 5.38(\mathrm{~m}, 2 \mathrm{H}), 3.72(\mathrm{~s}, 3 \mathrm{H}), 3.71(\mathrm{~s}, 3 \mathrm{H}), 3.69(\mathrm{~s}, 3 \mathrm{H})$, $3.68(\mathrm{~s}, 3 \mathrm{H}), 2.99(\mathrm{~m}, 2 \mathrm{H}), 2.05(\mathrm{br} \mathrm{d}, J=13.4 \mathrm{~Hz}, 2 \mathrm{H}), 1.93(\mathrm{~m}, 4 \mathrm{H}), 1.83(\mathrm{~m}, 2 \mathrm{H}), 1.72(\mathrm{~m}, 4$ H), $1.67(\mathrm{~d}, J=2.9 \mathrm{~Hz}, 3 \mathrm{H}), 1.63(\mathrm{~d}, J=2.9 \mathrm{~Hz}, 3 \mathrm{H}), 0.96(\mathrm{t}, J=7.4 \mathrm{~Hz}, 3 \mathrm{H}), 0.94$ (t, $J=7.4 \mathrm{~Hz}$, $3 \mathrm{H}) .{ }^{13} \mathrm{C} \mathrm{NMR}\left(\mathrm{CDCl}_{3}, 100 \mathrm{MHz}\right): \delta 201.3(2 \mathrm{C}), 170.3$ (4C), $166.2(2 \mathrm{C}), 135.1(2 \mathrm{C}), 133.1,133.0$, 130.9 (2C), 129.9 (2C), 129.7 (2C), 128.5 (4C), 125.7, 125.6, 106.1 (2C), 89.2 (2C), 66.1 (2C), 61.9, 61.8, 52.7 (2C), 52.5 (2C), 41.8, 41.5, 28.3 (2C), 27.1, 27.0, 20.2 (2C), 18.8, 18.4, 12.2 (2C).

Allene cis-6 was prepared as a 1:1 mixture of diastereoisomers from cis-2a and bromoallene 35d as described for cis-3a in 20\% yield. ${ }^{1} \mathrm{H}$ NMR $\left(\mathrm{CDCl}_{3}, 400 \mathrm{MHz}\right): \delta 6.02(\mathrm{~m}, 2 \mathrm{H}), 5.79(\mathrm{~m}, 4 \mathrm{H})$, 5.60 (sextet, $J=2.9 \mathrm{~Hz}, 1 \mathrm{H}$ ), 5.56 (sextet, $J=2.9 \mathrm{~Hz}, 1 \mathrm{H}), 5.10(\mathrm{~m}, 2 \mathrm{H}), 5.03-4.92(\mathrm{~m}, 4 \mathrm{H}), 3.73$ $(\mathrm{s}, 3 \mathrm{H}), 3.72(\mathrm{~s}, 3 \mathrm{H}), 3.70(\mathrm{~s}, 3 \mathrm{H}), 3.70(\mathrm{~s}, 3 \mathrm{H}), 2.95(\mathrm{~m}, 2 \mathrm{H}), 2.11(\mathrm{tq}, J=7.9,1.5 \mathrm{~Hz}, 4 \mathrm{H}), 2.02$ $(\mathrm{m}, 4 \mathrm{H}), 1.86(\mathrm{~m}, 2 \mathrm{H}), 1.73-1.52$ (overlapping peaks including two $\mathrm{d}$ at 1.68 and $1.66(\mathrm{~J}=2.9 \mathrm{~Hz})$, total $12 \mathrm{H}), 1.16(\mathrm{~s}, 9 \mathrm{H}), 1.15(\mathrm{~s}, 9 \mathrm{H}) .{ }^{13} \mathrm{C} \mathrm{NMR}\left(\mathrm{CDCl}_{3}, 100 \mathrm{MHz}\right): \delta 201.7(2 \mathrm{C}), 178.1(2 \mathrm{C})$, 170.2 (2C), 138.3, 138.2, 134.6 134.5, 126.2 (2C), 125.9 (2C), 115.0 (2C), 103.8 (2C), 89.0, 88.9, $65.6(2 \mathrm{C}), 61.8,61.7,52.8,52.7,52.5(2 \mathrm{C}), 41.7,41.4,38.9(2 \mathrm{C}), 33.3,33.2,32.6,31.8,31.7,28.1$, $27.3(3 \mathrm{C}), 27.2(3 \mathrm{C}), 24.6,20.1,18.9,18.5$.

Allene 7 was prepared from 36 and bromoallene 35a as described for cis-3a in $45 \%$ yield. ${ }^{1} \mathrm{H}$ $\operatorname{NMR}\left(\mathrm{CDCl}_{3}, 400 \mathrm{MHz}\right): \delta 5.65(\mathrm{td}, J=5.1,2.5 \mathrm{~Hz}, 2 \mathrm{H}), 5.47$ (septet, $\left.J=2.9 \mathrm{~Hz}, 1 \mathrm{H}\right), 4.46(\mathrm{~m}, 2$ $\mathrm{H}), 3.71(\mathrm{~s}, 6 \mathrm{H}), 2.72(\mathrm{~m}, 2 \mathrm{H}), 1.68(\mathrm{~d}, J=2.9 \mathrm{~Hz}, 6 \mathrm{H}), 1.18(\mathrm{~s}, 9 \mathrm{H}) .{ }^{13} \mathrm{C} \mathrm{NMR}\left(\mathrm{CDCl}_{3}, 100\right.$ $\mathrm{MHz}): \delta 201.9,178.4,170.7$ (2C), 129.4, 128.5, 100.4, 88.4, 64.7, 58.3, 52.8 (2C), 38.9, 37.4, 27.4 (3C), $20.2(2 \mathrm{C})$.

Allene cis-8a was prepared from cis-2d and bromoallene 35a as described for cis-3a in 57\% yield (86\% based on recovered sm). ${ }^{1} \mathrm{H}$ NMR $\left(\mathrm{CDCl}_{3}, 400 \mathrm{MHz}\right): \delta 5.65$ (ddd, $\left.J=11.7,4.9,2.3 \mathrm{~Hz}, 1 \mathrm{H}\right)$, $5.40(\mathrm{dt}, J=11.7,2.4 \mathrm{~Hz}, 1 \mathrm{H}), 5.40-5.34(\mathrm{~m}, 2 \mathrm{H}), 3.62(\mathrm{~s}, 6 \mathrm{H}), 2.92(\mathrm{~m}, 1 \mathrm{H}), 2.0-1.6$ (m including br s at $1.60,12 \mathrm{H}), 1.09(\mathrm{~s}, 9 \mathrm{H}) .{ }^{13} \mathrm{C} \mathrm{NMR}\left(\mathrm{CDCl}_{3}, 100 \mathrm{MHz}\right): \delta 202.1,177.3,170.1,170.0,132.8$, $131.8,99.5,87.6,73.4,62.1,52.3$ (2C), 44.2, 38.3, 32.1, 29.3, 27.5, 27.1 (3C), 19.9, 19.8. 
Allene cis-8b was prepared from cis-2e and bromoallene 35a as described for cis-3a in $61 \%$ yield. ${ }^{1} \mathrm{H}$ NMR $\left(\mathrm{CDCl}_{3}, 400 \mathrm{MHz}\right): \delta 5.71$ (ddd, $\left.J=11.5,4.9,2.4 \mathrm{~Hz}, 1 \mathrm{H}\right), 5.61$ (dddd, $J=11.5$, $3.5,2.4 ; 1.1 \mathrm{~Hz}, 1 \mathrm{H}), 5.47$ (br d, $J=11.0 \mathrm{~Hz}, 1 \mathrm{H}$ ), 5.43 (septet, $J=2.9 \mathrm{~Hz}, 1 \mathrm{H}$ ), $3.71(\mathrm{~s}, 6 \mathrm{H}$ ), 2.99 (dddt, $J=10.8,4.9,2.2 ; 1.1 \mathrm{~Hz}, 1 \mathrm{H}), 2.03(\mathrm{~s}, 3 \mathrm{H}), 1.99(\mathrm{~m}, 1 \mathrm{H}), 1.81(\mathrm{~m}, 1 \mathrm{H}), 1.72(\mathrm{~m}, 4$ $\mathrm{H}), 1.68(\mathrm{~d}, J=2.9 \mathrm{~Hz}, 3 \mathrm{H}), 1.67(\mathrm{~d}, J=2.9 \mathrm{~Hz}, 3 \mathrm{H}), 1.50(\mathrm{~m}, 1 \mathrm{H}), 1.19(\mathrm{~m}, 1 \mathrm{H}) .{ }^{13} \mathrm{C}$ NMR $\left(\mathrm{CDCl}_{3}, 100 \mathrm{MHz}\right): \delta 202.6,170.6,170.5,170.4,133.2,132.2,100.0,88.0,74.4,62.5,52.8,52.7$, 44.6, 32.5, 29.5, 27.8, 21.,5 20.2, 20.0.

Allene cis-8c was prepared from cis-2f and bromoallene 35a as described for cis-3a in $51 \%$ yield. ${ }^{1} \mathrm{H} \mathrm{NMR}\left(\mathrm{CDCl}_{3}, 400 \mathrm{MHz}\right.$ ): $\delta 8.04$ (br d, $\left.J=8.6 \mathrm{~Hz}, 2 \mathrm{H}\right), 7.55$ (br t, $J=7.5 \mathrm{~Hz}, 1 \mathrm{H}$ ), 7.43 (br t, $J$ $=7.5 \mathrm{~Hz}, 2 \mathrm{H}), 5.79(\mathrm{~m}, 2 \mathrm{H}), 5.75(\mathrm{br} \mathrm{d}, J=11.0, \mathrm{~Hz}, 1 \mathrm{H}), 5.47$ (septet, $J=2.9 \mathrm{~Hz}, 1 \mathrm{H}), 3.77$ (s, $6 \mathrm{H}), 3.08$ (br d, $J=10.6 \mathrm{~Hz}, 1 \mathrm{H}), 2.08(\mathrm{~m}, 1 \mathrm{H}), 1.99(\mathrm{~m}, 1 \mathrm{H}), 1.81(\mathrm{~m}, 2 \mathrm{H}), 1.71(\mathrm{~d}, J=2.9 \mathrm{~Hz}$, $3 \mathrm{H}), 1.70(\mathrm{~d}, J=2.9 \mathrm{~Hz}, 3 \mathrm{H}), 1.66(\mathrm{~m}, 1 \mathrm{H}), 1.28(\mathrm{~m}, 1 \mathrm{H}) .{ }^{13} \mathrm{C} \mathrm{NMR}\left(\mathrm{CDCl}_{3}, 100 \mathrm{MHz}\right): \delta 202.7$, 170.7, 170.6, 166.0, 133.1, 133.0, 132.5, 130.8, 129.8 (2C), 128.5 (2C), 100.1, 88.0, 74.9, 62.6, 52.8 (2C), 44.6, 32.5, 29.6, 27.8, 20.2, 20.1.

Allene trans-8a was prepared from trans-2d and bromoallene 35a as described for cis-3a in $77 \%$ yield. ${ }^{1} \mathrm{H} \mathrm{NMR}\left(\mathrm{CDCl}_{3}, 400 \mathrm{MHz}\right): \delta 5.83(\mathrm{dd}, J=11.5,3.5 \mathrm{~Hz}, 1 \mathrm{H}), 5.68(\mathrm{ddd}, J=11.5,4.8,2.5$, $\mathrm{Hz}, 1 \mathrm{H}), 5.45$ (septet, $J=2.9 \mathrm{~Hz}, 1 \mathrm{H}), 5.22(\mathrm{~m}, 1 \mathrm{H}), 3.72(\mathrm{~s}, 3 \mathrm{H}), 3.71(\mathrm{~s}, 3 \mathrm{H}), 3.23$ (m, 1H), 2.01.3 (m including d at $1.69 \mathrm{ppm}(2.9 \mathrm{~Hz})$ and d at $1.67 \mathrm{ppm}(2.9 \mathrm{~Hz}), 12 \mathrm{H}), 1.21(\mathrm{~s}, 9 \mathrm{H}) .{ }^{13} \mathrm{C} \mathrm{NMR}$ $\left(\mathrm{CDCl}_{3}, 100 \mathrm{MHz}\right): \delta 202.0,177.2,170.1,170.0,136.0,129.3,99.2,87.7,70.5,62.3,52.3,52.2$, 42.7, 38.6, 30.3, 29.3, 27.0 (3C), 23.8, 19.8, 19.7.

Allene trans-8b was prepared from trans-2e and bromoallene 35a as described for cis-3a in $63 \%$ yield. ${ }^{1} \mathrm{H} \mathrm{NMR}\left(\mathrm{CDCl}_{3}, 400 \mathrm{MHz}\right): \delta 5.77$ (ddt, $\left.J=11.8,3.3,1.1 \mathrm{~Hz}, 1 \mathrm{H}\right), 5.62$ (ddd, $J=11.8,3.6$, $2.5 \mathrm{~Hz}, 1 \mathrm{H}$ ), 5.46 (septet, $J=3.0 \mathrm{~Hz}, 1 \mathrm{H}$ ), 5.32 (br d, $J=9.3 \mathrm{~Hz}, 1 \mathrm{H}$ ), 3.72 (s, $6 \mathrm{H}$ ), 3.19 (br. d, $J$ $=11.0 \mathrm{~Hz}, 1 \mathrm{H}), 2.09(\mathrm{~s}, 3 \mathrm{H}), 1.98(\mathrm{~m}, 1 \mathrm{H}), 1.78(\mathrm{~m}, 4 \mathrm{H}), 1.68(\mathrm{~d}, J=3.0 \mathrm{~Hz}, 3 \mathrm{H}), 1.67(\mathrm{~d}, J=$ $3.0 \mathrm{~Hz}, 3 \mathrm{H}), 1.43(\mathrm{~m}, 1 \mathrm{H}) .{ }^{13} \mathrm{C} \mathrm{NMR}\left(\mathrm{CDCl}_{3}, 100 \mathrm{MHz}\right): \delta 202.6,170.6(2 \mathrm{C}), 170.5,135.5,130.1$, 99.8, 88.0, 71.8, 62.9, 52.8 (2C), 42.7, 30.9, 29.5, 23.4, 21.5, 20.2, 20.1.

Allene trans-8c was prepared from trans-2c and bromoallene 35a as described for cis-3a in $89 \%$ yield. ${ }^{1} \mathrm{H} \mathrm{NMR}\left(\mathrm{CDCl}_{3}, 400 \mathrm{MHz}\right): \delta 8.06($ br d, $J=7.3 \mathrm{~Hz}, 2 \mathrm{H}), 7.54($ br t, $J=7.3 \mathrm{~Hz}, 1 \mathrm{H}), 7.43$ (br t, $J=7.3 \mathrm{~Hz}, 2 \mathrm{H}$ ), 5.83 (dd, $J=11.7,3.1 \mathrm{~Hz}, 1 \mathrm{H}$ ), 5.79 (ddd, $J=11.7,4.2,2.3 \mathrm{~Hz}, 1 \mathrm{H}$ ), 5.56 $(\mathrm{m}, 1 \mathrm{H}), 5.47$ (septet, $J=2.9 \mathrm{~Hz}, 1 \mathrm{H}), 3.72(\mathrm{~s}, 6 \mathrm{H}), 3.32(\mathrm{br} \mathrm{d}, J=11.2 \mathrm{~Hz}, 1 \mathrm{H}), 2.12(\mathrm{~m}, 1 \mathrm{H})$, $1.87(\mathrm{~m}, 4 \mathrm{H}), 1.61(\mathrm{~d}, J=2.9 \mathrm{~Hz}, 3 \mathrm{H}), 1.59(\mathrm{~d}, J=2.9 \mathrm{~Hz}, 3 \mathrm{H}), 1.47(\mathrm{~m}, 1 \mathrm{H}) .{ }^{13} \mathrm{C} \mathrm{NMR}\left(\mathrm{CDCl}_{3}\right.$, $100 \mathrm{MHz}): \delta 202.6,170.7,170.6,166.0,136.3,133.0,130.9,129.8$ (2C), 129.7, 128.6 (2C), 99.6, $88.1,72.1,62.8,52.8(2 \mathrm{C}), 43.0,31.0,29.8,24.0,20.1,20.0$.

Allene cis $\mathbf{- 9}$ was prepared from cis-38 and bromoallene 35a as described for cis-3a in $84 \%$ yield. ${ }^{1} \mathrm{H} \mathrm{NMR}\left(\mathrm{CDCl}_{3}, 400 \mathrm{MHz}\right): \delta 5.99(\mathrm{ddd}, J=5.8,1.8,1.1 \mathrm{~Hz}, 1 \mathrm{H}), 5.59(\mathrm{dt}, J=5.8,2.2 \mathrm{~Hz}, 1 \mathrm{H})$, $5.59(\mathrm{~m}, 1 \mathrm{H}), 5.38$ (septet, $J=2.9 \mathrm{~Hz}, 1 \mathrm{H}), 3.71(\mathrm{~s}, 6 \mathrm{H}), 3.46(\mathrm{~m}, 1 \mathrm{H}), 2.55(\mathrm{dt}, J=14.6,8.4 \mathrm{~Hz}$, $1 \mathrm{H}), 1.67(\mathrm{~d}, J=2.9 \mathrm{~Hz}, 3 \mathrm{H}), 1.65(\mathrm{~d}, J=2.9 \mathrm{~Hz}, 3 \mathrm{H}), 1.60(\mathrm{ddd}, J=14.6,6.2,4.8 \mathrm{~Hz}, 1 \mathrm{H}), 1.16$ (s, 9H). ${ }^{13} \mathrm{C} \mathrm{NMR}\left(\mathrm{CDCl}_{3}, 100 \mathrm{MHz}\right): \delta 202.1,178.2,170.1$ (2C), 136.6, 130.8, 99.3, 86.9, 78.6, 61.4, 52.4, 52.3, 47.5, 38.4, 32.4, 27.0 (3C), 19.9, 19.8.

General Procedure for the Preparation of Allenes cis-10 and cis-11. Allenes cis-10. To a solution of $40(0.085 \mathrm{~g}, 0.4 \mathrm{mmol})$ in THF $(1 \mathrm{~mL})$ was added $\mathrm{NaH}(60 \%$ in mineral oil, $0.016 \mathrm{~g}, 0.4$ $\mathrm{mmol}$ ) and the mixture was stirred for $10 \mathrm{~min}$. The resulting sodium salt was transferred to a solution of $\mathrm{PPh}_{3}(0.008 \mathrm{~g}, 0.03 \mathrm{mmol})$ and $\mathrm{Pd}(\mathrm{OAc})_{2}(0.002 \mathrm{~g}, 0.008 \mathrm{mmol})$ in THF $(2 \mathrm{~mL})$. Chloropivalate cis $\mathbf{- 1}(\mathrm{n}=1, \mathrm{R}=\mathrm{Piv})(0.11 \mathrm{~g}, 0.52 \mathrm{mmol})$ was added and the mixture was stirred for 4 $\mathrm{h}$ at room temperature. The reaction mixture was partitioned between $\mathrm{Et}_{2} \mathrm{O}(20 \mathrm{~mL})$ and $\mathrm{H}_{2} \mathrm{O}(20$ 
$\mathrm{mL})$. The aqueous phase was extracted with $\mathrm{Et}_{2} \mathrm{O}(3 \times 10 \mathrm{~mL})$ and the combined organic phases were dried $\left(\mathrm{Na}_{2} \mathrm{SO}_{4}\right)$. The solvent was removed by evaporation followed by flash chromatography (pentane:Et $\left.{ }_{2} \mathrm{O}, 75: 25\right)$ to give $0.11 \mathrm{~g}(70 \%)$ of cis-10. ${ }^{1} \mathrm{H}$ NMR $\left(\mathrm{CDCl}_{3}, 400 \mathrm{MHz}\right): \delta 6.03(\mathrm{dt}, J=$ 10.4, $1.6 \mathrm{~Hz}, 1 \mathrm{H}), 5.79$ (dddd, $J=10.4,4.9,2.7,1.4 \mathrm{~Hz}, 1 \mathrm{H}), 5.07(\mathrm{~m}, 1 \mathrm{H}), 4.78(\mathrm{~m}, 1 \mathrm{H}), 3.73(\mathrm{~s}$, $3 \mathrm{H}), 3.66(\mathrm{~s}, 3 \mathrm{H}), 2.91(\mathrm{~m}, 1 \mathrm{H}), 2.67(\mathrm{dd}, J=14.3,8.2 \mathrm{~Hz}, 1 \mathrm{H}), 2.59(\mathrm{dd}, J=14.3,7.2 \mathrm{~Hz}, 1 \mathrm{H})$, $1.88(\mathrm{~m}, 1 \mathrm{H}), 1.68(\mathrm{~m}, 2 \mathrm{H}), 1.64(\mathrm{~s}, 3 \mathrm{H}), 16.3(\mathrm{~s}, 3 \mathrm{H}), 1.49(\mathrm{~m}, 1 \mathrm{H}), 1.15(\mathrm{~s}, 9 \mathrm{H}) .{ }^{13} \mathrm{C}$ NMR $\left(\mathrm{CDCl}_{3}, 100 \mathrm{MHz}\right): \delta 203.9,178.1,171.1,170.6,135.1,125.2,95.5,83.0,65.5,61.2,52.5,52.1$, 38.9, 38.8, 33.2, 28.1, 27.3 (3C), $20.6(2 \mathrm{C}), 19.1$.

Allenes cis-11 was prepared from cis-5 and $\mathbf{4 0}$ as described for cis-10 in 92\% yield. ${ }^{1} \mathrm{H}$ NMR $\left(\mathrm{CDCl}_{3}, 300 \mathrm{MHz}\right): \delta 5.70(\mathrm{ddd}, J=11.3,5.1,2.2 \mathrm{~Hz}, 1 \mathrm{H}), 5.59(\mathrm{dt}, J=11.7,2.6 \mathrm{~Hz}, 1 \mathrm{H}), 5.43$ (br d, $J=11.0,1 \mathrm{H}), 4.78(\mathrm{~m}, 1 \mathrm{H}), 3.66(\mathrm{~s}, 6 \mathrm{H}), 2.92(\mathrm{dd}, J=10.6,5.1 \mathrm{~Hz}, 1 \mathrm{H}), 2.53(\mathrm{~d}, J=7.7$ $\mathrm{Hz}, 2 \mathrm{H}), 1.98(\mathrm{~m}, 1 \mathrm{H}), 1.74(\mathrm{~m}, 3 \mathrm{H}), 1.59(\mathrm{~d}, J=2.9 \mathrm{~Hz}, 3 \mathrm{H}), 1.58(\mathrm{~d}, J=2.9 \mathrm{~Hz}, 3 \mathrm{H}), 1.45(\mathrm{~m}$, $1 \mathrm{H}), 1.13(\mathrm{~s}, 9 \mathrm{H}) 1.08(\mathrm{~m}, 1 \mathrm{H}) .{ }^{13} \mathrm{C} \mathrm{NMR}\left(\mathrm{CDCl}_{3}, 100 \mathrm{MHz}\right): \delta 203.5,177.4,170.7$ (2C), 133.6, $130.9,94.8,82.9,73.4,61.2,52.0,51.9,42.9,38.3,34.2,31.8,28.9,27.6,26.9$ (3C), 20.3, 20.2.

\section{Preparation of compound cis-12:}

Cyclization of allene cis-3b was preformed as described for the cyclization of cis-3a to give cis12 in $88 \%$ yield.

Cyclization of allene cis-3c was preformed as described for the cyclization of cis-3a to give cis12 in $89 \%$ yield.

Preparation of compound cis-13: Spectral data was in accordance with those previously reported (Franzén, J. Bäckvall J. E. J. Am. Chem. Soc. 2003, 125, 6056-6057).

Cyclization of allene cis-4a was preformed as described for the cyclization of cis-3a to give cis13 in $68 \%$ yield.

Cyclization of allene cis-4b was preformed as described for the cyclization of cis-3a to give cis13 in $76 \%$ yield.

Cyclization of allene cis-4c was preformed as described for the cyclization of cis-3a to give cis13 in $92 \%$ yield.

Preparation of compound cis-14: Spectral data was in accordance with those previously reported (Franzén, J. Bäckvall J. E. J. Am. Chem. Soc. 2003, 125, 6056-6057).

Cyclization of allene cis-5a was preformed as described for the cyclization of cis-3a to give cis14a and cis-14b as a 1:1 mixture of regioisomers in $66 \%$ total yield.

Cyclization of allene cis-5b was preformed as described for the cyclization of cis-3a to give cis$\mathbf{1 4 a}$ and cis-14b as a 1:1 mixture of regioisomers in $92 \%$ total yield.

Cyclization of allene cis-5c was preformed as described for the cyclization of cis-3a to give cis14a and cis-14b as a 1:1 mixture of regioisomers in $95 \%$ total yield.

Compound cis-15 was prepared from cis-6 as described for the cyclization cis-3a in $68 \%$ yield. ${ }^{1} \mathrm{H}$ NMR $\left(\mathrm{CDCl}_{3}, 400 \mathrm{MHz}\right): \delta 5.89$ (dddd, $\left.J=10.4,3.9,2.2,1.4 \mathrm{~Hz}, 1 \mathrm{H}\right), 5.79(\mathrm{~m}, 2 \mathrm{H}), 5.68(\mathrm{~d}, J=$ $2.5 \mathrm{~Hz}, 1 \mathrm{H}), 5.05(\mathrm{~s}, 2 \mathrm{H}), 4.99$ (ddd, $J=16.3,3.6,1.6 \mathrm{~Hz}, 1 \mathrm{H}), 4.94(\mathrm{ddt}, J=10.1,2.2,1.1 \mathrm{~Hz}, 1 \mathrm{H})$, $3.74(\mathrm{~s}, 3 \mathrm{H}), 3.70(\mathrm{~s}, 3 \mathrm{H}), 3.65(\mathrm{~m}, 1 \mathrm{H}), 3.24$ (ddd, $J=11.3,6.6,4.9 \mathrm{~Hz}, 1 \mathrm{H}), 2.43-2.11(\mathrm{~m}, 4 \mathrm{H})$, 
$1.96(\mathrm{~m}, 2 \mathrm{H}), 1.44(\mathrm{~m}, 2 \mathrm{H}) .{ }^{13} \mathrm{C} \mathrm{NMR}\left(\mathrm{CDCl}_{3}, 100 \mathrm{MHz}\right): \delta 171.0,170.5,150.2,142.9,138.3$, 128.3, 126.1, 122.9, 115.0, 114.1, 68.7, 52.9, 52.5, 44.6, 42.7, 34.6, 32.6, 23.5, 21.9. - Anal. Calcd. for $\mathrm{C}_{19} \mathrm{H}_{24} \mathrm{O}_{4}: \mathrm{C}, 72.13 ; \mathrm{H}, 7.65$. Found: $\mathrm{C}, 72.25 ; \mathrm{H}, 7.59$.

Compound 16 was prepared from $\mathbf{7}$ as described for the cyclization cis-3a in 59\% yield with the exceptions that acetonitrile was used as solvent and the reaction mixture was refluxed for $5 \mathrm{~h} .{ }^{1} \mathrm{H}$ NMR $\left(\mathrm{CDCl}_{3}, 400 \mathrm{MHz}\right): \delta 5.83(\mathrm{~d}, J=1.4 \mathrm{~Hz}, 1 \mathrm{H}), 5.74(\mathrm{ddd}, J=17.1,10.1,8.6 \mathrm{~Hz}, 1 \mathrm{H}), 5.14$ (br s, $1 \mathrm{H}$ ), 5.04 (ddd, $J=17.1,1.7,0.9 \mathrm{~Hz}, 1 \mathrm{H}$ ), 5.04 (quintet, $J=1.4 \mathrm{~Hz}, 1 \mathrm{H}$ ), 4.98 (ddd, $J=$ 10.1, 1.6, $0.7 \mathrm{~Hz}, 1 \mathrm{H}$ ), 3.73 (s, $6 \mathrm{H}), 3.65$ (td, $J=8.6,4.2 \mathrm{~Hz}, 1 \mathrm{H}), 2.89$ (dd, $J=13.8,8.6 \mathrm{~Hz}, 1 \mathrm{H}$ ), $2.34(\mathrm{dd}, J=13.8,4.2 \mathrm{~Hz}, 1 \mathrm{H}), 1.93(\mathrm{dd}, J=1.4,0.7 \mathrm{~Hz}, 3 \mathrm{H}) \cdot{ }^{13} \mathrm{C} \mathrm{NMR}\left(\mathrm{CDCl}_{3}, 100 \mathrm{MHz}\right): \delta$ 171.8, 171.7, 149.8, 141.5, 138.1, 125.4, 116.9, 115.1, 65.4, 53.1, 53.0, 49.1, 39.4, 21.4. - Anal. Calcd. for $\mathrm{C}_{14} \mathrm{H}_{18} \mathrm{O}_{4}: \mathrm{C}, 67.18 ; \mathrm{H}, 7.25$. Found: $\mathrm{C}, 67.41 ; \mathrm{H}, 7.40$.

\section{Preparation of compound cis-17:}

Cyclization of allene cis-8b was preformed as described for the cyclization of cis-3a to give cis17 in $83 \%$ yield with the exceptions that $5 \mathrm{~mol} \% \mathrm{Pd}(\mathrm{dba})_{2}$ was employed, decane was used as solvent and the reaction mixture was refluxed for $2 \mathrm{~h} .{ }^{1} \mathrm{H}$ NMR $\left(\mathrm{CDCl}_{3}, 400 \mathrm{MHz}\right): \delta 5.75$ (br s, 1 H), 5.53 (dddd, $J=10.4,7.0,5.1,2.7 \mathrm{~Hz}, 1 \mathrm{H}$ ), 5.40 (ddd, $J=10.4,4.5,2.3 \mathrm{~Hz}, 1 \mathrm{H}$ ), 5.07 (br s, 1H), 5.00 (br s, 1H), $3.92(\mathrm{~m}, 1 \mathrm{H}), 3.76(\mathrm{~s}, 3 \mathrm{H}), 3.67$ (s, 3H), 3.07 (ddd, $J=12.6,9.4,5.0, \mathrm{~Hz}, 1 \mathrm{H})$, $2.34(\mathrm{~m}, 1 \mathrm{H}), 2.12(\mathrm{~m}, 1 \mathrm{H}), 1.95(\mathrm{~s}, 3 \mathrm{H}), 1.87(\mathrm{~m}, 1 \mathrm{H}), 1.62(\mathrm{~m}, 1 \mathrm{H}), 1.32(\mathrm{~m}, 2 \mathrm{H}) .{ }^{13} \mathrm{C}$ NMR $\left(\mathrm{CDCl}_{3}, 100 \mathrm{MHz}\right): \delta 171.4,171.1,151.3,138.0,130.6,126.4,124.8,116.3,69.9,52.6,52.1,47.0$, 45.6, 26.4, 24.9 21.0, 20.6. - Anal. Calcd. for $\mathrm{C}_{17} \mathrm{H}_{22} \mathrm{O}_{4}:$ C, 70.32; H, 7.64. Found: C, 70.41; H, 7.60.

Cyclization of allene trans-8a was preformed as described for the cyclization of cis-3a to give cis-17 in $88 \%$ yield with the exceptions that acetonitrile was used as solvent and the reaction mixture was refluxed for $10 \mathrm{~h}$.

Cyclization of allene trans-8b was preformed as described for the cyclization of cis-3a to give cis-17 in 91\% yield with the exceptions that acetonitrile was used as solvent and the reaction mixture was refluxed for $10 \mathrm{~h}$.

Compound trans-17 was prepared from trans-8b as described for the cyclization of cis-3b to give a mixture of trans-17 and trans-19 (ratio 93:7) in 90\% total yield with the exceptions that 5 $\operatorname{mol} \% \operatorname{Pd}(\mathrm{dba})_{2}$ was employed, decane was used as solvent and the reaction mixture was refluxed for $2 \mathrm{~h}$. . Compound trans $\mathbf{- 1 7}$ and trans $\mathbf{- 1 9}$ was separated by HPLC. Spectral data for trans $\mathbf{- 1 7}$ was in accordance with those previously reported (Franzén, J. Bäckvall J. E. J. Am. Chem. Soc. 2003, 125, 6056-6057). trans-19: ${ }^{1} \mathrm{H}$ NMR $\left(\mathrm{CDCl}_{3}, 300 \mathrm{MHz}\right): \delta 5.88(\mathrm{~m}, 2 \mathrm{H}), 5.45$ (br s, $\left.1 \mathrm{H}\right), 3.74(\mathrm{~s}, 3 \mathrm{H}) 3.6$ $(\mathrm{s}, 3 \mathrm{H}), 2.51(\mathrm{br} \mathrm{d}, J=9.3 \mathrm{~Hz}, 1 \mathrm{H}), 2.6-1.2(\mathrm{~m}, 8 \mathrm{H}), 1.14(\mathrm{~d}, J=6.6,3 \mathrm{H}), 1.01(\mathrm{~d}, J=6.9,3 \mathrm{H}) .{ }^{13} \mathrm{C}$ $\mathrm{NMR}\left(\mathrm{CDCl}_{3}, 75 \mathrm{MHz}\right): \delta 171.1$ (2C), 158.0, 132.7, 132.4, 120.8, 68.2, 52.6, 52.4, 51.6, 50.0, 32.3, $28.1,27.7,26.2,21.5,20.6$.

Compound cis-21b was prepared from cis-3b as described for cis-21a in $71 \%$ yield with the exception that the reaction mixture was refluxed for $0.3 \mathrm{~h} .{ }^{1} \mathrm{H} \mathrm{NMR}\left(\mathrm{CDCl}_{3}, 400 \mathrm{MHz}\right): \delta 5.85$ (ddt, $J=10.1,4.6,2.2 \mathrm{~Hz}, 1 \mathrm{H}), 5.77(\mathrm{dtd}, J=10.1,3.7,2.2 \mathrm{~Hz}, 1 \mathrm{H}), 5.59(\mathrm{dd}, J=3.1,0.8 \mathrm{~Hz}, 1 \mathrm{H})$, $3.73(\mathrm{~s}, 3 \mathrm{H}), 3.69$ (s, $3 \mathrm{H}), 3.48(\mathrm{~m}, 1 \mathrm{H}), 3.06$ (dd, $J=16.8,5.9 \mathrm{~Hz}, 1 \mathrm{H}), 1.98$ (m, $2 \mathrm{H}), 1.92$ (s, 3 $\mathrm{H}), 1.56(\mathrm{~s}, 3 \mathrm{H}), 1.55(\mathrm{~s}, 3 \mathrm{H}), 1.31(\mathrm{~m}, 2 \mathrm{H}) .{ }^{13} \mathrm{C} \mathrm{NMR}\left(\mathrm{CDCl}_{3}, 100 \mathrm{MHz}\right): \delta 170.6,170.1,169.8$, $153.4,128.6,126.4,120.9,79.9,68.1,52.9,52.4,45.1,43.7,27.3,27.2,24.3,22.2,21.4$.

Compound cis-21c was prepared from cis-3c as described for cis-21a in 64\% yield with the exception that the reaction mixture was refluxed for $0.3 \mathrm{~h} .{ }^{1} \mathrm{H} \mathrm{NMR}\left(\mathrm{CDCl}_{3}, 400 \mathrm{MHz}\right): \delta 7.96$ (br d, 
$J=8.5 \mathrm{~Hz}, 2 \mathrm{H}), 7.52$ (br t, $J=7.5 \mathrm{~Hz}, 1 \mathrm{H}), 7.40($ br t $, J=7.5 \mathrm{~Hz}, 2 \mathrm{H}), 5.84$ (ddt, $J=10.3,4.6$, $2.2 \mathrm{~Hz}, 1 \mathrm{H}), 5.70(\mathrm{dd}, J=2.9,0.7 \mathrm{~Hz}, 1 \mathrm{H}), 5.62$ (br. d, $J=10.1 \mathrm{~Hz}, 1 \mathrm{H}), 3.75$ (s, $3 \mathrm{H}), 3.73(\mathrm{~s}, 3$ H), $3.58(\mathrm{~m}, 1 \mathrm{H}), 3.06(\mathrm{dt}, J=16.8,5.7 \mathrm{~Hz}, 1 \mathrm{H}), 1.95(\mathrm{~m}, 2 \mathrm{H}), 1.74(\mathrm{~s}, 3 \mathrm{H}), 1.72(\mathrm{~s}, 3 \mathrm{H}), 1.36$ $(\mathrm{m}, 2 \mathrm{H}) .{ }^{13} \mathrm{C}$ NMR $\left(\mathrm{CDCl}_{3}, 100 \mathrm{MHz}\right): \delta 170.7,170.1,165.2,154.2,132.8,131.6,129.7,128.4$, $128.2,126.4,121.0,80.8,68.2,53.0,52.4,45.3,43.7,27.5,27.1,24.3,21.5$.

Compound cis-22a was prepared from cis-4a as described for cis-21a in $77 \%$ yield. ${ }^{1} \mathrm{H}$ NMR $\left(\mathrm{CDCl}_{3}, 400 \mathrm{MHz}\right): \delta 5.87(\mathrm{ddt}, J=10.1,4.6,2.2 \mathrm{~Hz}, 1 \mathrm{H}), 5.73(, J=\mathrm{Hz}, 1 \mathrm{H}), 5.57$ (dd, $J=2.9$, $0.7 \mathrm{~Hz}, 1 \mathrm{H}), 3.72$ (s, $3 \mathrm{H}), 3.69(\mathrm{~s}, 3 \mathrm{H}), 3.40(\mathrm{~m}, 1 \mathrm{H}), 3.04(\mathrm{td}, J=8.4,6.0 \mathrm{~Hz}, 1 \mathrm{H}), 2.38$ (broad $\mathrm{d}, J=2.38 \mathrm{~Hz}, 2 \mathrm{H}), 1.96(\mathrm{~m}, 2 \mathrm{H}), 1.64($ broad d, $J=12.6 \mathrm{~Hz}, 1 \mathrm{H}), 1.55(\mathrm{~m}, 4 \mathrm{H}), 1.45$ (dt, $J=$ 12.6, $3.4 \mathrm{~Hz}, 1 \mathrm{H}), 1.41-1.28(\mathrm{~m}, 4 \mathrm{H}), 1.18(\mathrm{~s}, 9 \mathrm{H}) .{ }^{13} \mathrm{C} \mathrm{NMR}\left(\mathrm{CDCl}_{3}, 100 \mathrm{MHz}\right): \delta 176.6,170.8$, 170.1, 153.9, 128.0, 127.0, 121.0, 80.5, 68.2, 52.9, 52.4, 45.1, 43.4, 39.7, 35.8, 33.8, 27.7 (3C), 25.5, 24.3, 21.8 (2C) 21.6. - Anal. Calcd. for $\mathrm{C}_{21} \mathrm{H}_{30} \mathrm{O}_{6}$ : C, 68.87; H, 8.19. Found: $\mathrm{C}$, 68.940; H, 8.39 .

Compound cis-22b was prepared from cis-4b as described for cis-21a in $67 \%$ yield with the exception that the reaction mixture was refluxed for $0.3 \mathrm{~h} .{ }^{1} \mathrm{H} \mathrm{NMR}\left(\mathrm{CDCl}_{3}, 400 \mathrm{MHz}\right): \delta 5.87$ (ddt, $J=10.1,4.2,2.2 \mathrm{~Hz}, 1 \mathrm{H}), 5.76$ (br. d, $J=10.1 \mathrm{~Hz}, 1 \mathrm{H}), 5.62(\mathrm{dd}, J=2.9,0.7 \mathrm{~Hz}, 1 \mathrm{H}), 3.74(\mathrm{~s}, 3$ H), $3.70(\mathrm{~s}, 3 \mathrm{H}), 3.47(\mathrm{~m}, 1 \mathrm{H}), 3.05(\mathrm{dt}, J=11.2,5.9 \mathrm{~Hz}, 1 \mathrm{H}), 2.41(\mathrm{~m}, 1 \mathrm{H}), 2.29(\mathrm{~m}, 1 \mathrm{H}), 1.98$ $(\mathrm{m}, 2 \mathrm{H}), 1.96(\mathrm{~s}, 3 \mathrm{H}), 1.63(\mathrm{~m}, 1 \mathrm{H}), 1.52(\mathrm{~m}, 6 \mathrm{H}), 1.28(\mathrm{~m}, 3 \mathrm{H}) \cdot{ }^{13} \mathrm{C} \mathrm{NMR}\left(\mathrm{CDCl}_{3}, 100 \mathrm{MHz}\right): \delta$ 170.6, 170.2, 169.7, 153.5, 127.8, 126.6, 121.5, 81.0, 68.3, 52.9, 52.4, 45.0, 43.5, 35.1, 34.5, 25.5, 24.3, 22.0, 21.9, 21.7, 21.5.

Compound cis-22c was prepared from cis-4c as described for cis-21a in 54\% yield with the exception that the reaction mixture was refluxed for $0.3 \mathrm{~h} .{ }^{1} \mathrm{H} \mathrm{NMR}\left(\mathrm{CDCl}_{3}, 400 \mathrm{MHz}\right): \delta 8.00$ (br d, $J=8.4 \mathrm{~Hz}, 2 \mathrm{H}$ ), 7.53 (br t, $J=7.5 \mathrm{~Hz}, 1 \mathrm{H}), 7.42$ (br t, $J=7.5 \mathrm{~Hz}, 2 \mathrm{H}), 5.82$ (ddt, $J=10.3,4.8$, $2.2 \mathrm{~Hz}, 1 \mathrm{H}), 5.69$ (dd, $J=2.9,0.8 \mathrm{~Hz}, 1 \mathrm{H}$ ), 5.54 (br. d, $J=10.3 \mathrm{~Hz}, 1 \mathrm{H}), 3.75$ (s, $3 \mathrm{H}), 3.73$ (s, 3 H), $3.51(\mathrm{~m}, 1 \mathrm{H}), 3.05$ (ddd, $J=12.6,5.9,4.2 \mathrm{~Hz}, 1 \mathrm{H}), 2.56$ (br t, $J=13.5 \mathrm{~Hz}, 2 \mathrm{H}), 1.91(\mathrm{~m}, 2 \mathrm{H})$, $1.65(\mathrm{~m}, 7 \mathrm{H}), 1.32(\mathrm{~m}, 3 \mathrm{H}) .{ }^{13} \mathrm{C} \mathrm{NMR}\left(\mathrm{CDCl}_{3}, 100 \mathrm{MHz}\right): \delta 170.7,170.2,164.8,153.9,132.7$, 131.6, 129.6 (2C), 128.5 (2C), 127.8, 126.6, 121.3, 81.6, 68.3, 52.9, 52.4, 45.2, 43.5, 35.9, 34.3, $25.8,24.3,22.0,21.9,21.5$.

Compound cis-23a was prepared as a 1:1 mixture of diastereoisomers from cis-5a as described for cis-21a in 45\% yield. ${ }^{1} \mathrm{H}$ NMR $\left(\mathrm{CDCl}_{3}, 300 \mathrm{MHz}\right)$ : $\delta 5.85(\mathrm{~m}, 2 \mathrm{H}), 5.77(\mathrm{~m}, 2 \mathrm{H}), 5.60(\mathrm{dd}, J=$ 2.9, $0.8 \mathrm{~Hz}, 1 \mathrm{H}), 5.57(\mathrm{dd}, J=2.9,0.8 \mathrm{~Hz}, 1 \mathrm{H}), 3.74(\mathrm{~s}, 3 \mathrm{H}), 3.74(\mathrm{~s}, 3 \mathrm{H}), 3.71(\mathrm{~s}, 3 \mathrm{H}), 3.69$ (s, 3 H), $3.48(\mathrm{~m}, 1 \mathrm{H}), 3.42(\mathrm{~m}, 1 \mathrm{H}), 3.08(\mathrm{~m}, 2 \mathrm{H}), 1.99(\mathrm{~m}, 5 \mathrm{H}), 1.96(\mathrm{dd}, J=14.3,6.4 \mathrm{~Hz}, 1 \mathrm{H}), 1.85$ $(\mathrm{dd}, J=13.9,7.5 \mathrm{~Hz}, 1 \mathrm{H}), 1.77(\mathrm{dd}, J=13.9,7.5 \mathrm{~Hz}, 1 \mathrm{H}), 1.56(\mathrm{~s}, 3 \mathrm{H}), 1.54(\mathrm{~s}, 3 \mathrm{H}), 1.34(\mathrm{~m}, 4$ $\mathrm{H}), 1.17(\mathrm{~s}, 9 \mathrm{H}), 1.15(\mathrm{~s}, 9 \mathrm{H}), 0.83(\mathrm{t}, J=7.5 \mathrm{~Hz}, 3 \mathrm{H}), 0.78(\mathrm{t}, J=7.5 \mathrm{~Hz}, 3 \mathrm{H}) .{ }^{13} \mathrm{C} \mathrm{NMR}\left(\mathrm{CDCl}_{3}\right.$, $75 \mathrm{MHz}): \delta 177.0,176.7,170.8,170.6,170.2$, 170.1, 153.0, 152.5, 128.3 (2C), 126.6, 126.4, 122.1, $121.7,82.7,82.2,68.2,68.1,52.9,52.8,52.4$ (2C), 45.3, 45.0, 43.5 (2C), 39.5, 39.4, 31.8, 31.7, $29.9,27.5$ (6C), 24.3, $23.2(2 \mathrm{C}), 21.8,21.5,8.17 .8$.

Compound cis-23b was prepared as a 1:1 mixture of diastereoisomers from cis-5b as described for cis-21a in $60 \%$ yield with the exception that the reaction mixture was refluxed for $0.3 \mathrm{~h}$. (Isomer 1); ${ }^{1} \mathrm{H} \mathrm{NMR}\left(\mathrm{CDCl}_{3}, 400 \mathrm{MHz}\right): \delta 5.84$ (ddt, $\left.J=10.1,4.6,1.9 \mathrm{~Hz}, 1 \mathrm{H}\right), 5.76$ (br. d, $J=10.1 \mathrm{~Hz}, 1$ H), $5.63(\mathrm{dd}, J=2.9,0.7 \mathrm{~Hz}, 1 \mathrm{H}), 3.74(\mathrm{~s}, 3 \mathrm{H}), 3.68(\mathrm{~s}, 3 \mathrm{H}), 3.46(\mathrm{~m}, 1 \mathrm{H}), 3.06$ (ddd, $J=12.8$, 5.8, 4.1 Hz, $1 \mathrm{H}), 1.98$ (m, $2 \mathrm{H}), 1.91(\mathrm{~s}, 3 \mathrm{H}), 1.87$ (dd, $J=13.8,7.4 \mathrm{~Hz}, 1 \mathrm{H}), 1.80$ (dd, $J=13.8$, $7.4 \mathrm{~Hz}, 1 \mathrm{H}), 1.59$ (s, $3 \mathrm{H}), 1.37(\mathrm{~m}, 2 \mathrm{H}), 0.75(\mathrm{t}, J=7.4 \mathrm{~Hz}, 3 \mathrm{H}) .{ }^{13} \mathrm{C} \mathrm{NMR}\left(\mathrm{CDCl}_{3}, 100 \mathrm{MHz}\right): \delta$ 170.5 (2C), 169.7, 152.2, 128.0, 126.1, 122.8, 82.7, 68.1, 52.8, 52.4, 44.8, 43.7, 31.9, 24.3, 22.9, 22.3, 21.3, 7.9. (Isomer 2); ${ }^{1} \mathrm{H} \mathrm{NMR}\left(\mathrm{CDCl}_{3}, 400 \mathrm{MHz}\right): \delta 5.84(\mathrm{ddt}, J=10.1,4.4,2.0 \mathrm{~Hz}, 1 \mathrm{H})$, 5.78 (br. d, $J=10.1 \mathrm{~Hz}, 1 \mathrm{H}), 5.62(\mathrm{dd}, J=2.9,0.7 \mathrm{~Hz}, 1 \mathrm{H}), 3.74(\mathrm{~s}, 3 \mathrm{H}), 3.71(\mathrm{~s}, 3 \mathrm{H}), 3.45(\mathrm{~m}, 1$ H), $3.08(\mathrm{dt}, J=11.7,5.7 \mathrm{~Hz}, 1 \mathrm{H}), 2.00(\mathrm{~m}, 3 \mathrm{H}), 1.98(\mathrm{~s}, 3 \mathrm{H}), 1.78(\mathrm{dd}, J=13.7,7.5 \mathrm{~Hz}, 1 \mathrm{H})$, 
$1.56(\mathrm{~s}, 3 \mathrm{H}), 1.33(\mathrm{~m}, 2 \mathrm{H}), 0.82(\mathrm{t}, J=7.4 \mathrm{~Hz}, 3 \mathrm{H}) .{ }^{13} \mathrm{C} \mathrm{NMR}\left(\mathrm{CDCl}_{3}, 100 \mathrm{MHz}\right): \delta 170.7,170.1$, $169.8,152.8,128.2$, 126.4, 121.9, 83.3, 68.2, 52.9, 52.4, 45.4, 43.6, 31.5, 24.3, 23.5, 22.1, 21.7, 8.1.

Compound cis-23c was prepared as a 1:1 mixture of diastereoisomers from cis-5c as described for cis-21a in 58\% yield with the exception that the reaction mixture was refluxed for $0.3 \mathrm{~h}$. (Isomer 1); ${ }^{1} \mathrm{H} \mathrm{NMR}\left(\mathrm{CDCl}_{3}, 400 \mathrm{MHz}\right.$ ): $\delta 7.95$ (br d, $\left.J=8.4 \mathrm{~Hz}, 2 \mathrm{H}\right), 7.52$ (br t, $\left.J=7.3 \mathrm{~Hz}, 1 \mathrm{H}\right), 7.40$ (br t, $J=7.3 \mathrm{~Hz}, 2 \mathrm{H}$ ), 5.81 (ddt, $J=10.3,4.8,2.2 \mathrm{~Hz}, 1 \mathrm{H}$ ), 5.72 (dd, $J=2.9,0.7 \mathrm{~Hz}, 1 \mathrm{H}$ ), 5.51 (br. d, $J=10.1 \mathrm{~Hz}, 1 \mathrm{H}), 3.76(\mathrm{~s}, 3 \mathrm{H}), 3.71(\mathrm{~s}, 3 \mathrm{H}), 3.54(\mathrm{~m}, 1 \mathrm{H}), 3.09$ (ddd, $J=13.0,5.9,4.0 \mathrm{~Hz}, 1 \mathrm{H})$, 2.11 (dd, $J=13.8,7.5 \mathrm{~Hz}, 1 \mathrm{H}), 2.01$ (dd, $J=13.8,7.5 \mathrm{~Hz}, 1 \mathrm{H}), 1.93(\mathrm{~m}, 2 \mathrm{H}), 1.73(\mathrm{~s}, 3 \mathrm{H}), 1.44$ $(\mathrm{m}, 1 \mathrm{H}), 1.32(\mathrm{~m}, 1 \mathrm{H}), 0.85(\mathrm{t}, J=7.5 \mathrm{~Hz}, 3 \mathrm{H}) \cdot{ }^{13} \mathrm{C} \mathrm{NMR}\left(\mathrm{CDCl}_{3}, 100 \mathrm{MHz}\right): \delta$ 170.6, 170.2, 165.1, 152.4, 132.6, 131.8, 129.6 (2C), 128.4 (2C), 128.2, 126.0, 122.7, 83.5, 68.2, 52.9, 52.5, 45.1, 43.6, 31.9, 24.2, 23.5, 21.4, 8.0. (Isomer 2); ${ }^{1} \mathrm{H} \mathrm{NMR}\left(\mathrm{CDCl}_{3}, 400 \mathrm{MHz}\right): \delta 8.00$ (br d, $J=8.2 \mathrm{~Hz}, 2$ H), 7.54 (br t, $J=7.3 \mathrm{~Hz}, 1 \mathrm{H}$ ), 7.42 (br t, $J=7.3 \mathrm{~Hz}, 2 \mathrm{H}$ ), 5.83 (ddt, $J=10.1,4.5,2.2 \mathrm{~Hz}, 1 \mathrm{H}$ ), 5.68 (overlapping peaks including a dd, $J=2.9,0.7 \mathrm{~Hz}$, total $2 \mathrm{H}), 3.76(\mathrm{~s}, 3 \mathrm{H}), 3.75(\mathrm{~s}, 3 \mathrm{H}), 3.52$ $(\mathrm{m}, 1 \mathrm{H}), 3.09(\mathrm{dt}, J=10.8,6.0 \mathrm{~Hz}, 1 \mathrm{H}), 2.18(\mathrm{dd}, J=13.9,7.3 \mathrm{~Hz}, 1 \mathrm{H}), 1.97(\mathrm{~m}, 2 \mathrm{H}), 1.91(\mathrm{dd}, J$ $=13.9,7.3 \mathrm{~Hz}, 1 \mathrm{H}), 1.73(\mathrm{~s}, 3 \mathrm{H}), 1.36(\mathrm{~m}, 2 \mathrm{H}), 0.92(\mathrm{t}, J=7.3 \mathrm{~Hz}, 3 \mathrm{H}) .{ }^{13} \mathrm{C} \mathrm{NMR}\left(\mathrm{CDCl}_{3}, 100\right.$ $\mathrm{MHz}): \delta 170.7,170.1,165.1,153.0,132.8,131.5,129.7,128.5,128.2,126.5,122.0,84.0,68.3$, 53.0, 52.4, 45.5, 43.6, 32.0, 24.3, 23.5, 21.8, 8.3.

Compound $\mathbf{3 4}$ was prepared from cis-13 as described for $\mathbf{3 3}$ in $94 \%$ yield. ${ }^{1} \mathrm{H}$ NMR $\left(\mathrm{CDCl}_{3}, 400\right.$ MHz): $\delta 5.84$ (ddt, $J=10.1,4.5,2.2 \mathrm{~Hz}, 1 \mathrm{H}), 5.67(\mathrm{~m}, 1 \mathrm{H}), 3.93(\mathrm{dd}, J=8.8,4.4 \mathrm{~Hz}, 1 \mathrm{H}), 3.74$ (s, $3 \mathrm{H}), 3.72(\mathrm{~s}, 3 \mathrm{H}), 3.45$ (heptet, $J=2.2 \mathrm{~Hz}, 1 \mathrm{H}), 3.39(\mathrm{~m}, 1 \mathrm{H}), 3.34(\mathrm{t}, J=8.8 \mathrm{~Hz}, 1 \mathrm{H}), 3.05$ $(\mathrm{ddd}, J=10.6,6.6,4.2 \mathrm{~Hz}, 1 \mathrm{H}), 2.47(\mathrm{~m}, 1 \mathrm{H}), 2.34(\mathrm{dt}, J=15.2,6.6 \mathrm{~Hz}, 1 \mathrm{H}), 2.21(\mathrm{~m}, 1 \mathrm{H}), 2.00$ $(\mathrm{m}, 2 \mathrm{H}), 1.87(\mathrm{~m}, 1 \mathrm{H}), 1.75(\mathrm{~m}, 1 \mathrm{H}), 1.55(\mathrm{~m}, 4 \mathrm{H}), 1.47(\mathrm{~m}, 2 \mathrm{H}) \cdot{ }^{13} \mathrm{C} \mathrm{NMR}\left(\mathrm{CDCl}_{3}, 400 \mathrm{MHz}\right): \delta$ $172.5,171.6,171.3,170.5,139.3,136.7,129.9,127.0,125.9,64.1,53.1,46.8,44.7,44.4$, 44.0, $42.4,36.9,26.0,25.5,23.7,22.2,22.1,21.9$.

Preparation of compound 36. To a solution of dimethyl malonate $(0.58 \mathrm{~mL}, 5.1 \mathrm{mmol})$ in THF $(20 \mathrm{~mL})$ was added $\mathrm{NaH}(60 \%$ in mineral oil, $0.2 \mathrm{~g}, 5.1 \mathrm{mmol})$ and the mixture was stirred for 10 min. To this mixture was added $\mathrm{Pd}(\mathrm{OAc})_{2}(0.031 \mathrm{~g}, 0.14 \mathrm{mmol})$ and $\mathrm{PPh}_{3}(0.12 \mathrm{~g}, 0.46 \mathrm{mmol})$ followed by 1,4-bispivalyloxy-2-butene. The resulting mixture was refluxed for $4 \mathrm{~h}$ and partitioned between $\mathrm{Et}_{2} \mathrm{O}(20 \mathrm{ml})$ and $\mathrm{H}_{2} \mathrm{O}(20 \mathrm{ml})$. The aqueous phase was extracted with $\mathrm{Et}_{2} \mathrm{O}(3 \times 10 \mathrm{ml})$ and the combined organic phases were dried $\left(\mathrm{Na}_{2} \mathrm{SO}_{4}\right)$. The solvent was removed in vacuo followed by flash chromatography (pentane: $\left.\mathrm{Et}_{2} \mathrm{O}, 90: 10\right)$ to give $1.33 \mathrm{~g}$ (57\% yield) of $\mathbf{3 6} .{ }^{1} \mathrm{H}$ NMR $\left(\mathrm{CDCl}_{3}, 400\right.$ $\mathrm{MHz}): \delta 5.68(\mathrm{dt}, J=15.5,5.9 \mathrm{~Hz}, 1 \mathrm{H}), 5.66(\mathrm{dt}, J=15.5,5.1 \mathrm{~Hz}, 1 \mathrm{H}), 4.47(\mathrm{br} \mathrm{d}, J=5.1 \mathrm{~Hz}, 2$ $\mathrm{H}), 3.73(\mathrm{~s}, 6 \mathrm{H}), 3.44(\mathrm{t}, J=7.6 \mathrm{~Hz}, 1 \mathrm{H}), 2.64(\mathrm{~m}, 2 \mathrm{H}), 1.18(\mathrm{~s}, 9 \mathrm{H}) .{ }^{13} \mathrm{C} \mathrm{NMR}\left(\mathrm{CDCl}_{3}, 100\right.$ $\mathrm{MHz}): \delta 178.4,169.3(2 \mathrm{C}), 130.1,127.8,64.5,52.8(2 \mathrm{C}), 51.5,38.9,31.7,27.4(3 \mathrm{C})$.

Preparation of compound cis-38. To a solution of cis-1,4-hydroxy malonate cis-37 (100 mg, 0.5 $\mathrm{mmol})$ in $\mathrm{CH}_{2} \mathrm{Cl}_{2}(2 \mathrm{~mL})$ at $0^{\circ} \mathrm{C}$ was added pyridine $(55 \mu \mathrm{l}, 0.7 \mathrm{mmol})$ and DMAP $(6 \mathrm{mg}, 0.05$ $\mathrm{mmol}$ ) and finally pivalyl chloride $(70 \mu \mathrm{l}, 0.6 \mathrm{mmol})$. The mixture was stirred for $4 \mathrm{~h}$ at $0^{\circ} \mathrm{C}$. The reaction mixture was diluted with $20 \mathrm{~mL} \mathrm{CH}_{2} \mathrm{Cl}_{2}$ washed with $2 \mathrm{M} \mathrm{HCl}(10 \mathrm{~mL})$ and sat. $\mathrm{NaHCO}_{3}$ $(10 \mathrm{~mL})$. The aqueous phase was extracted with $\mathrm{CH}_{2} \mathrm{Cl}_{2}(3 \times 10 \mathrm{~mL})$ and the combined organic phases were dried $\left(\mathrm{Na}_{2} \mathrm{SO}_{4}\right)$. The solvent was removed by evaporation followed by flash chromatography (pentane: $\left.\mathrm{Et}_{2} \mathrm{O}, 90: 10\right)$ to give $61 \mathrm{mg}$ (43\% yield) of cis-38. ${ }^{1} \mathrm{H}$ NMR $\left(\mathrm{CDCl}_{3}, 300\right.$ $\mathrm{MHz}$ ): $\delta 6.03$ (broad d, $J=10.2 \mathrm{~Hz}, 1 \mathrm{H}), 5.83$ (broad d, $J=10.2 \mathrm{~Hz}, 1 \mathrm{H}), 5.57(\mathrm{~m}, 1 \mathrm{H}), 3.73(\mathrm{~s}$, $6 \mathrm{H}), 3.28$ (brosd s, 2H), $2.55(\mathrm{~m}, 1 \mathrm{H}), 1.51(\mathrm{~m}, 1 \mathrm{H}), 1.12(\mathrm{~s}, 9 \mathrm{H}) .{ }^{13} \mathrm{C} \mathrm{NMR}\left(\mathrm{CDCl}_{3}, 75 \mathrm{MHz}\right): \delta$ $178.4,168.9,168.8,137.6,131.8,78.7,57.3,52.7$ (2C), 43.8, 38.7, 35.0, 27.3 (3C).

Preparation of Allene 40. To a solution of dimethyl malonate $(0.23 \mathrm{~mL}, 1.96 \mathrm{mmol})$ in THF $(2.5$ $\mathrm{mL}$ ) was added $\mathrm{NaH}(60 \%$ in mineral oil, $0.04 \mathrm{~g}, 1.1 \mathrm{mmol})$ and the mixture was stirred for $10 \mathrm{~min}$. 
To this was added $\mathrm{Pd}\left(\mathrm{PPh}_{3}\right)_{4}(23 \mathrm{mg}, 0.034 \mathrm{mmol})$ followed by allene $39(0.20 \mathrm{~g}, 1.0 \mathrm{mmol})$. The resulting mixture was stirred over night at $45{ }^{\circ} \mathrm{C}$. The reaction mixture were partitioned between $\mathrm{Et}_{2} \mathrm{O}(20 \mathrm{~mL})$ and $\mathrm{H}_{2} \mathrm{O}(20 \mathrm{~mL})$. The aqueous phase was extracted with $\mathrm{Et}_{2} \mathrm{O}(3 \times 10 \mathrm{~mL})$ and the combined organic phases were dried $\left(\mathrm{Na}_{2} \mathrm{SO}_{4}\right)$. The solvent was removed by evaporation followed by flash chromatography (pentane: $\left.\mathrm{Et}_{2} \mathrm{O}, 90: 10\right)$ to give $0.15 \mathrm{~g}$ (73\% yield) of $40 .{ }^{1} \mathrm{H} \mathrm{NMR}\left(\mathrm{CDCl}_{3}\right.$, $400 \mathrm{MHz}$ ): $\delta 4.98$ (heptet, $J=2.9 \mathrm{~Hz}, 1 \mathrm{H}), 3.72$ (s, $6 \mathrm{H}), 3.48$ (t, $J=7.5 \mathrm{~Hz}, 1 \mathrm{H}), 2.53$ (dd, $J=7.5$, $5.8 \mathrm{~Hz}, 2 \mathrm{H}), 1.63(\mathrm{~d}, J=2.9 \mathrm{~Hz}, 6 \mathrm{H}) .{ }^{13} \mathrm{C} \mathrm{NMR}\left(\mathrm{CDCl}_{3}, 100 \mathrm{MHz}\right): \delta 202.0,169.7(2 \mathrm{C}), 97.8,85.7$, 52.7 (2C), 51.4, 28.5, 20.7 (2C). 

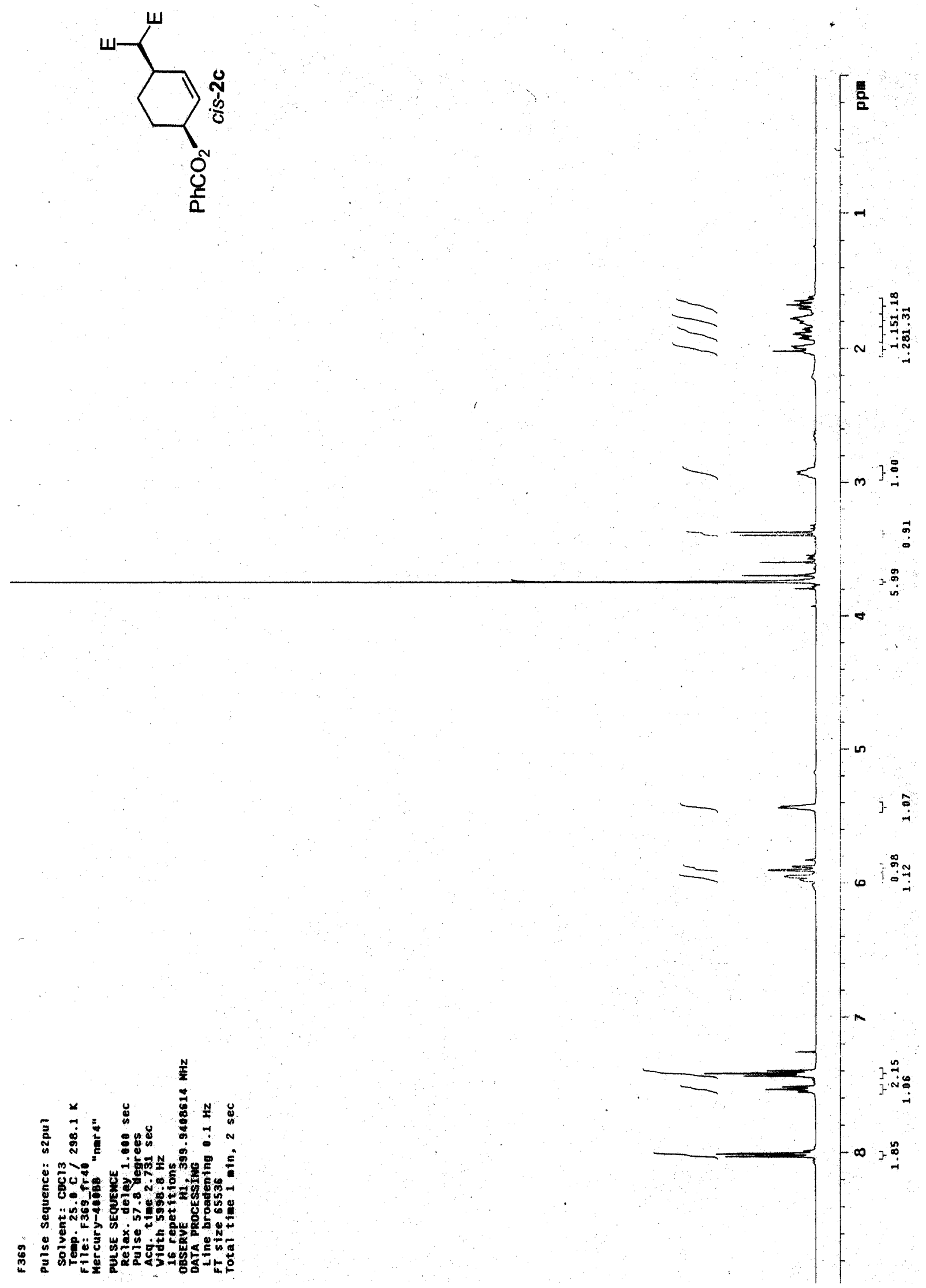

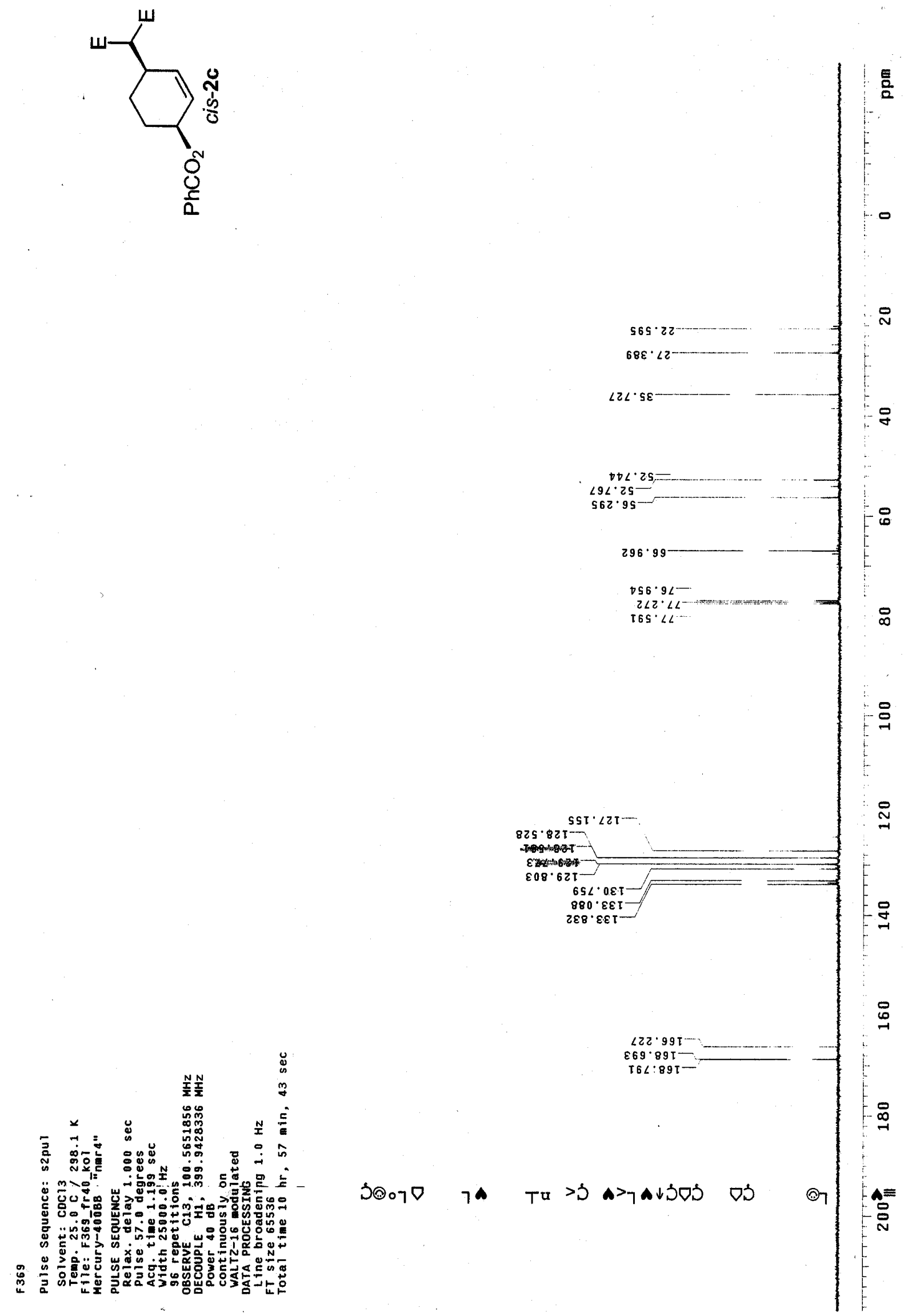


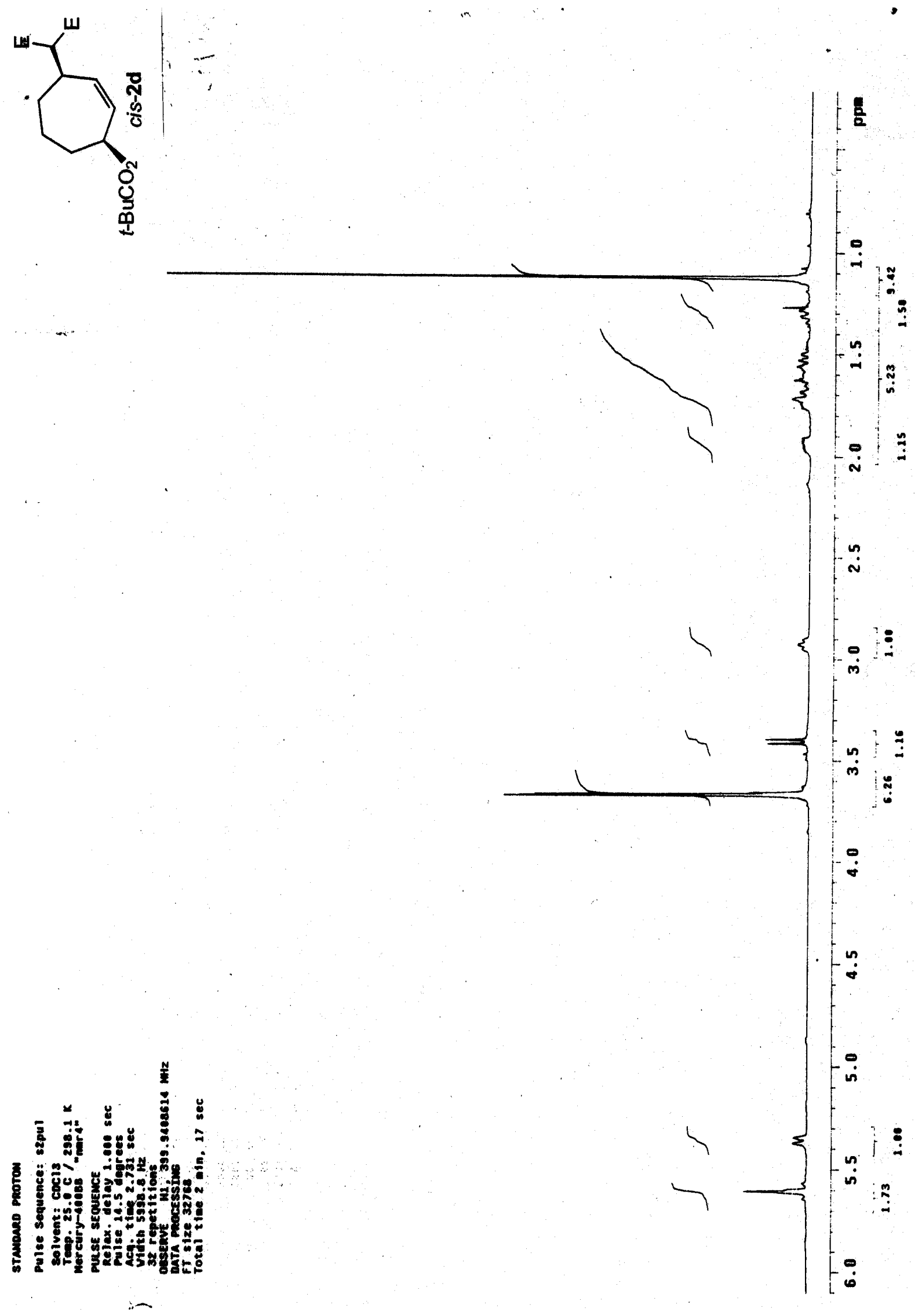



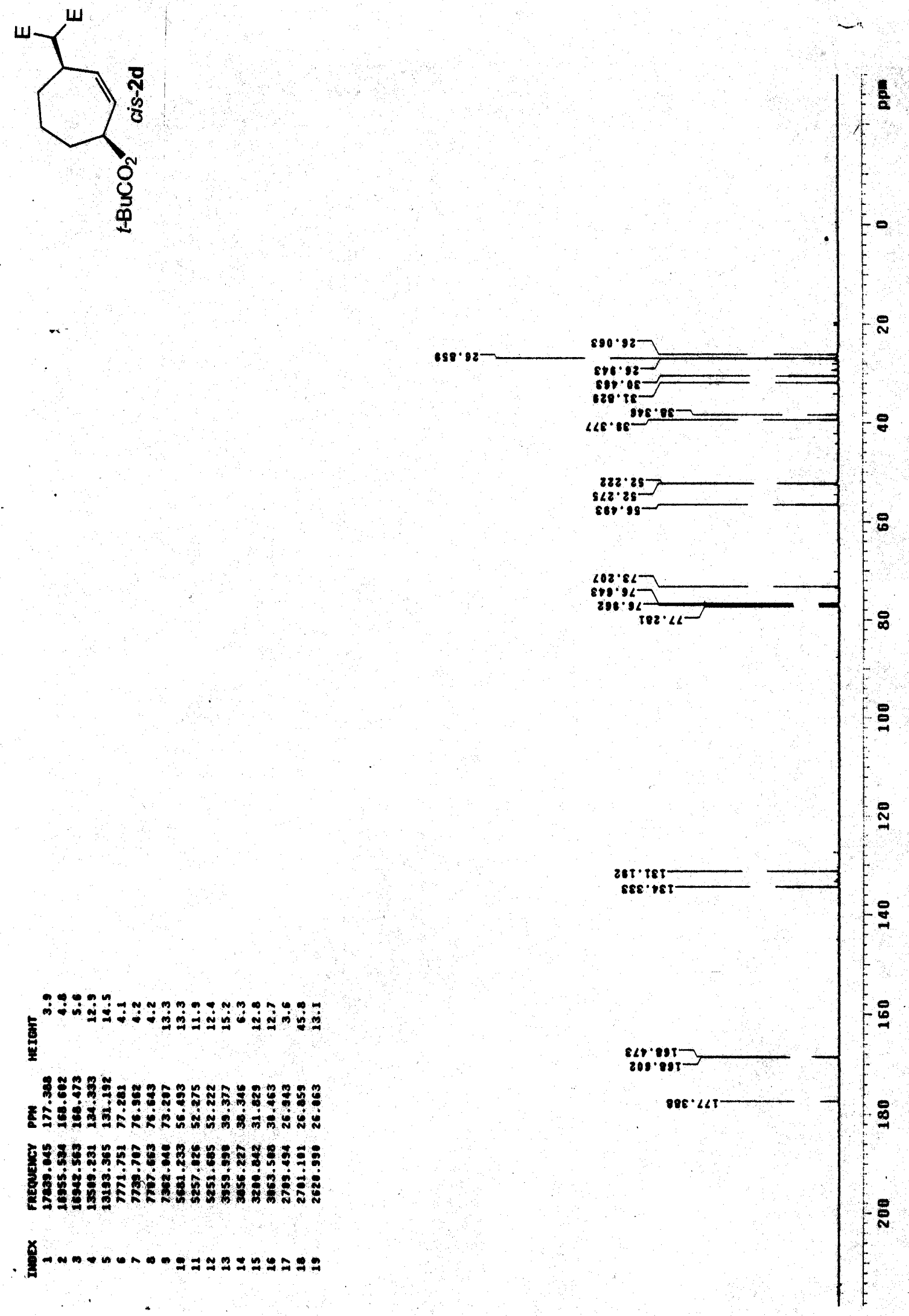


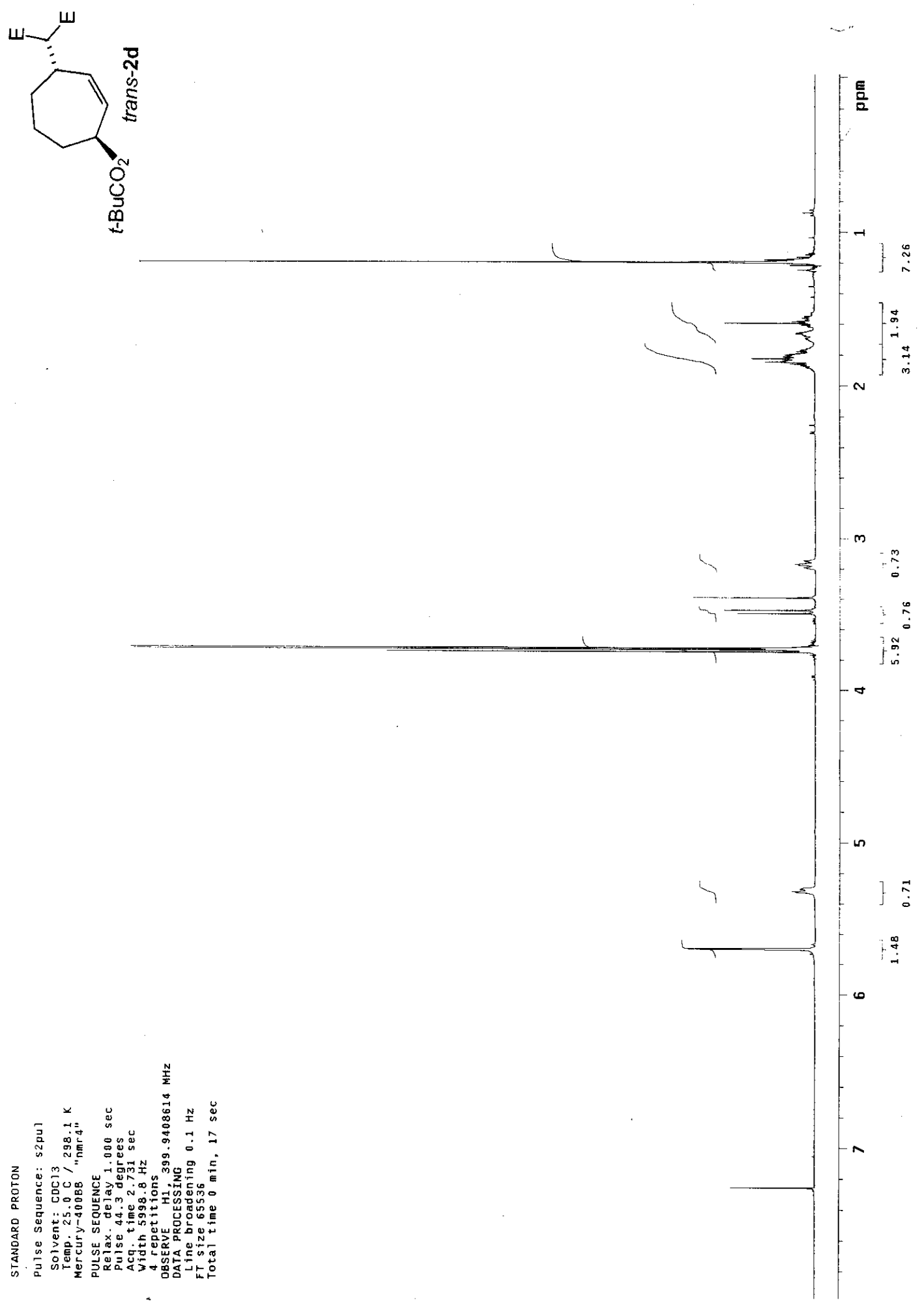



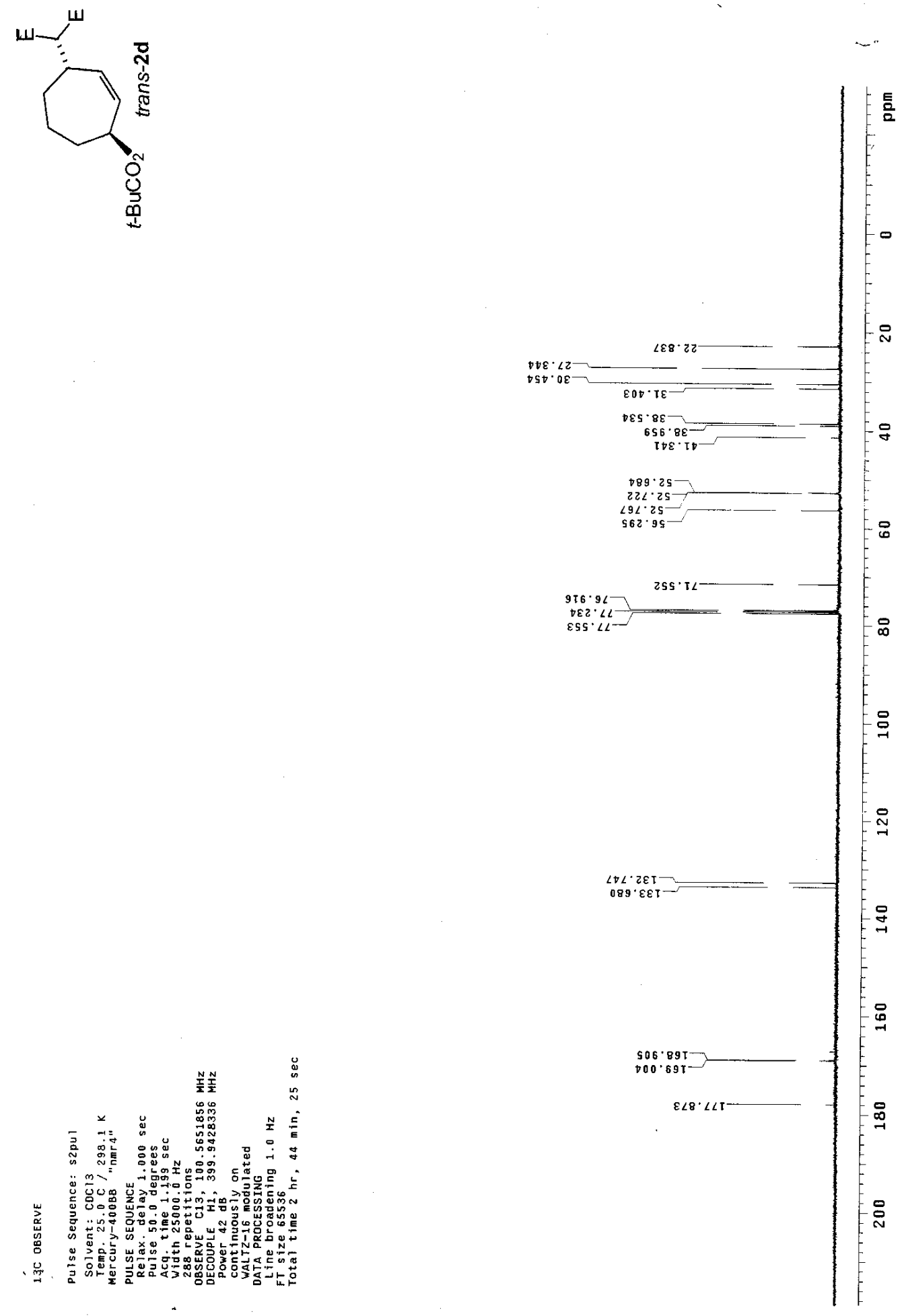

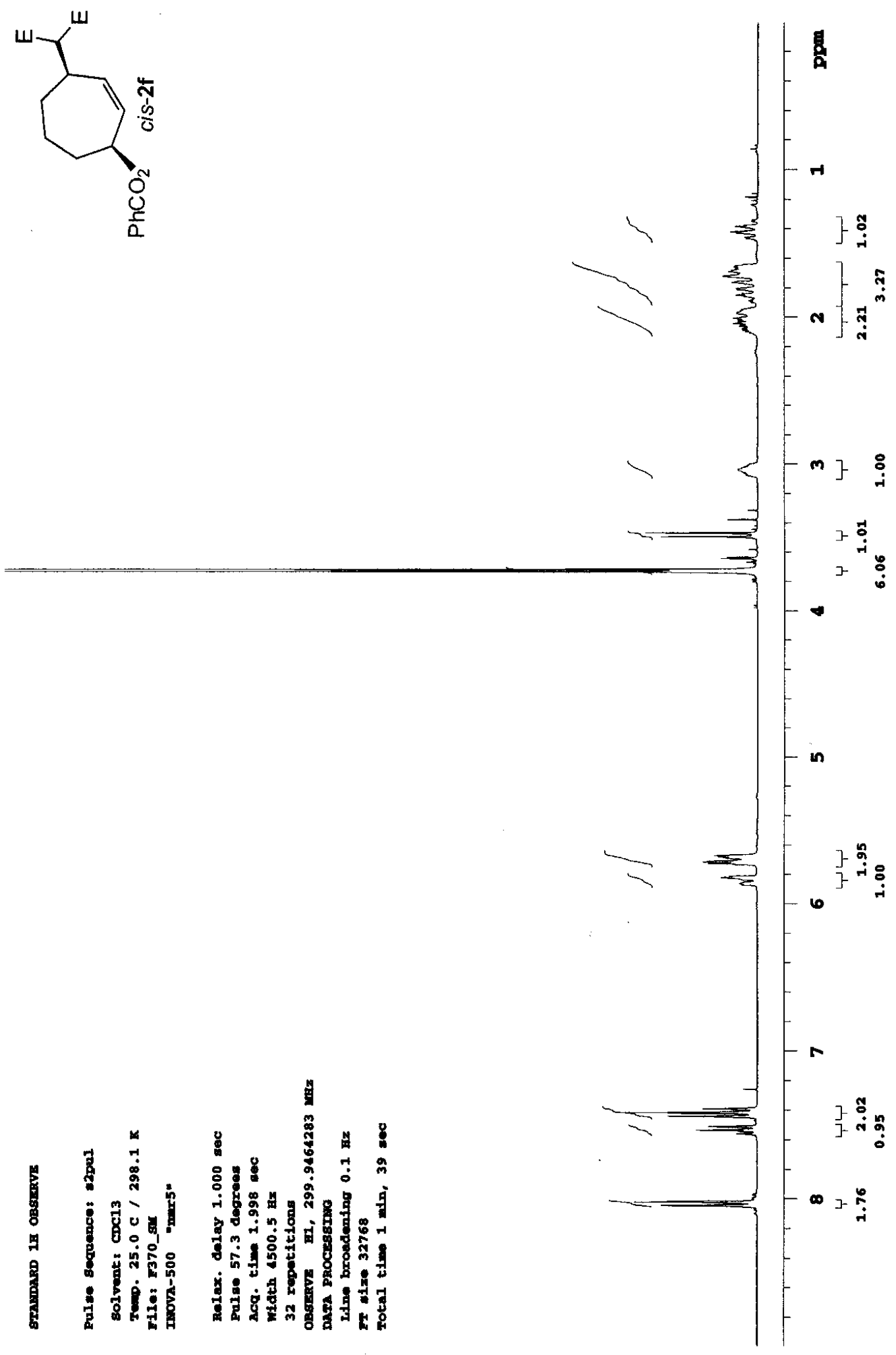

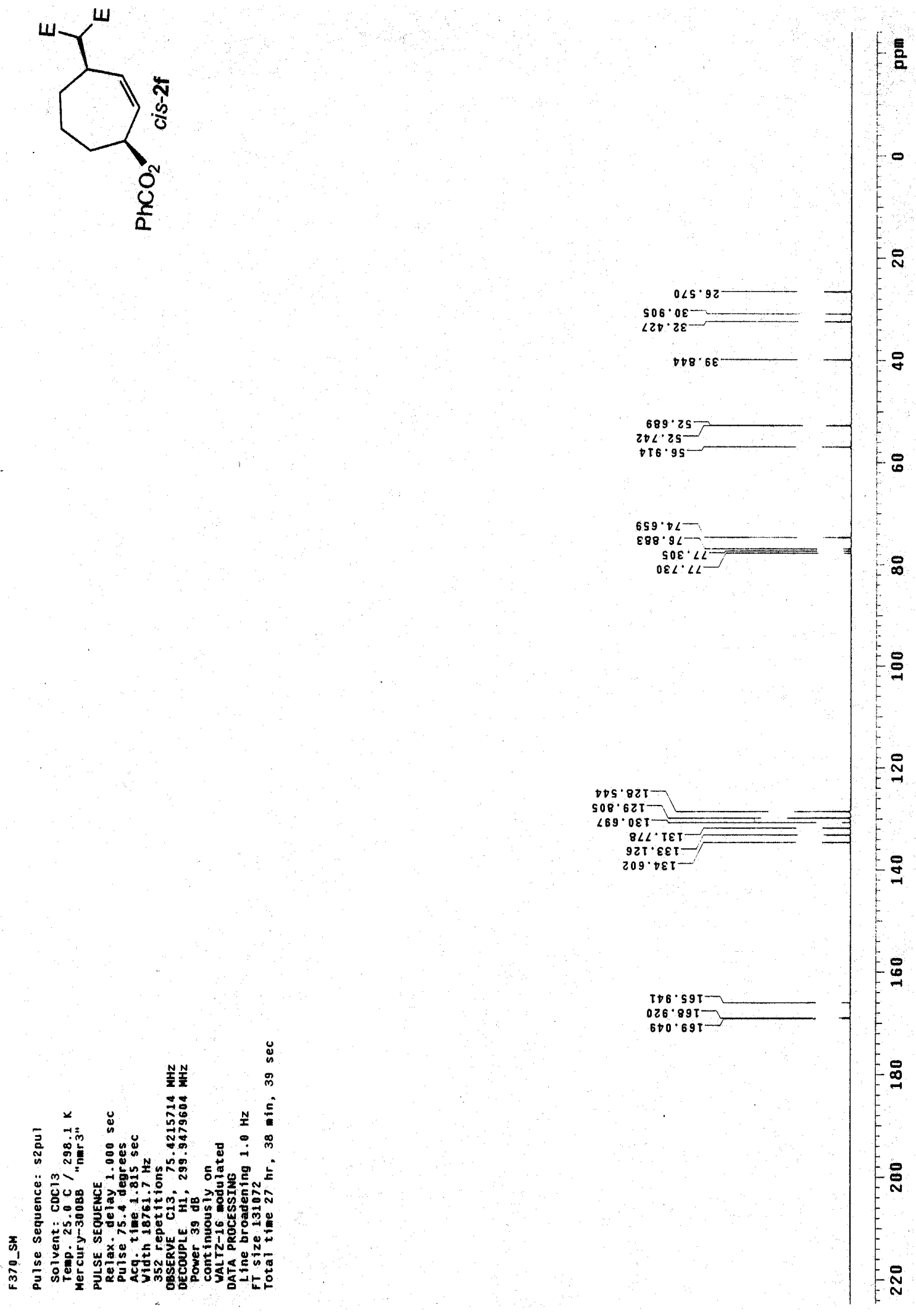

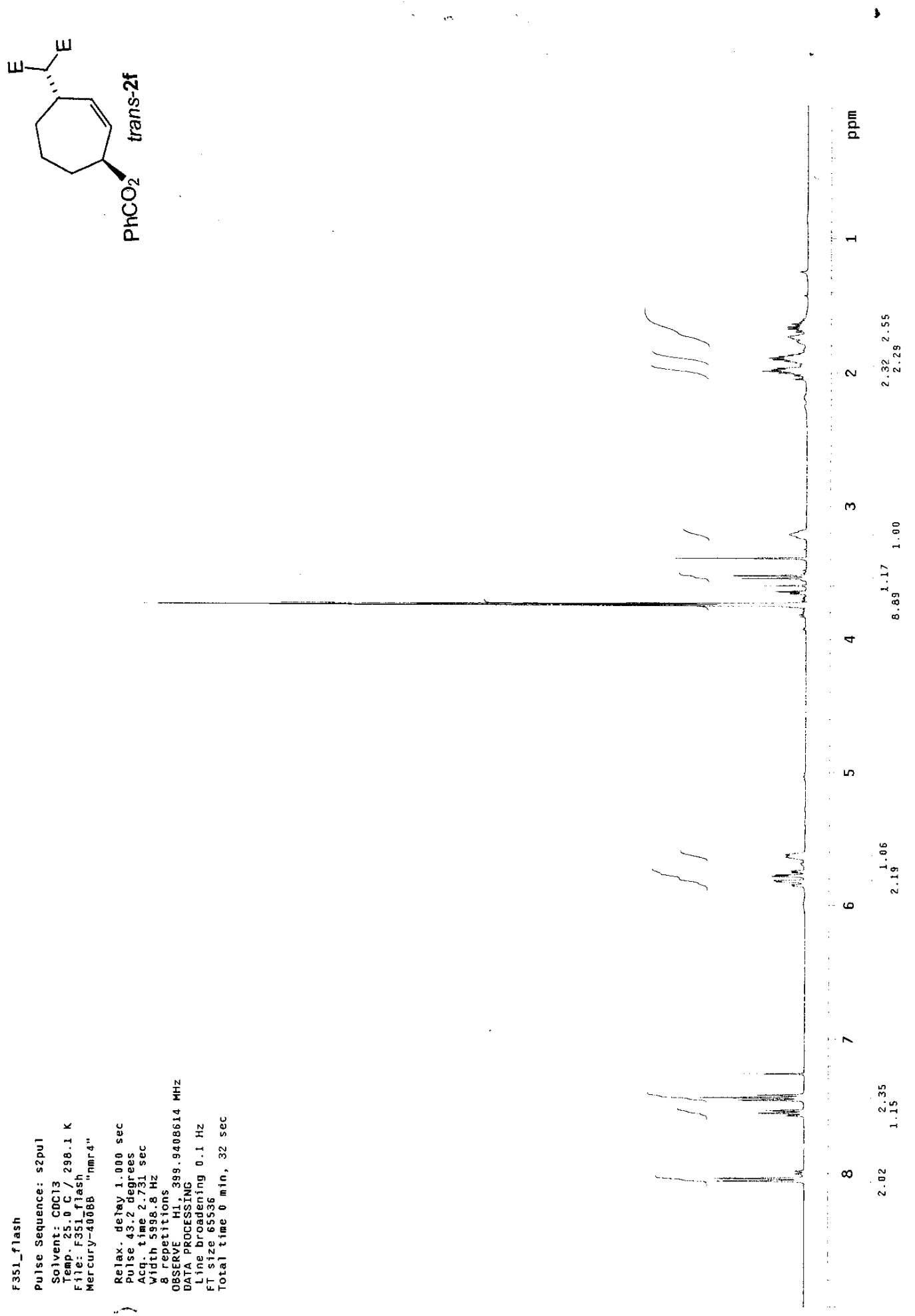


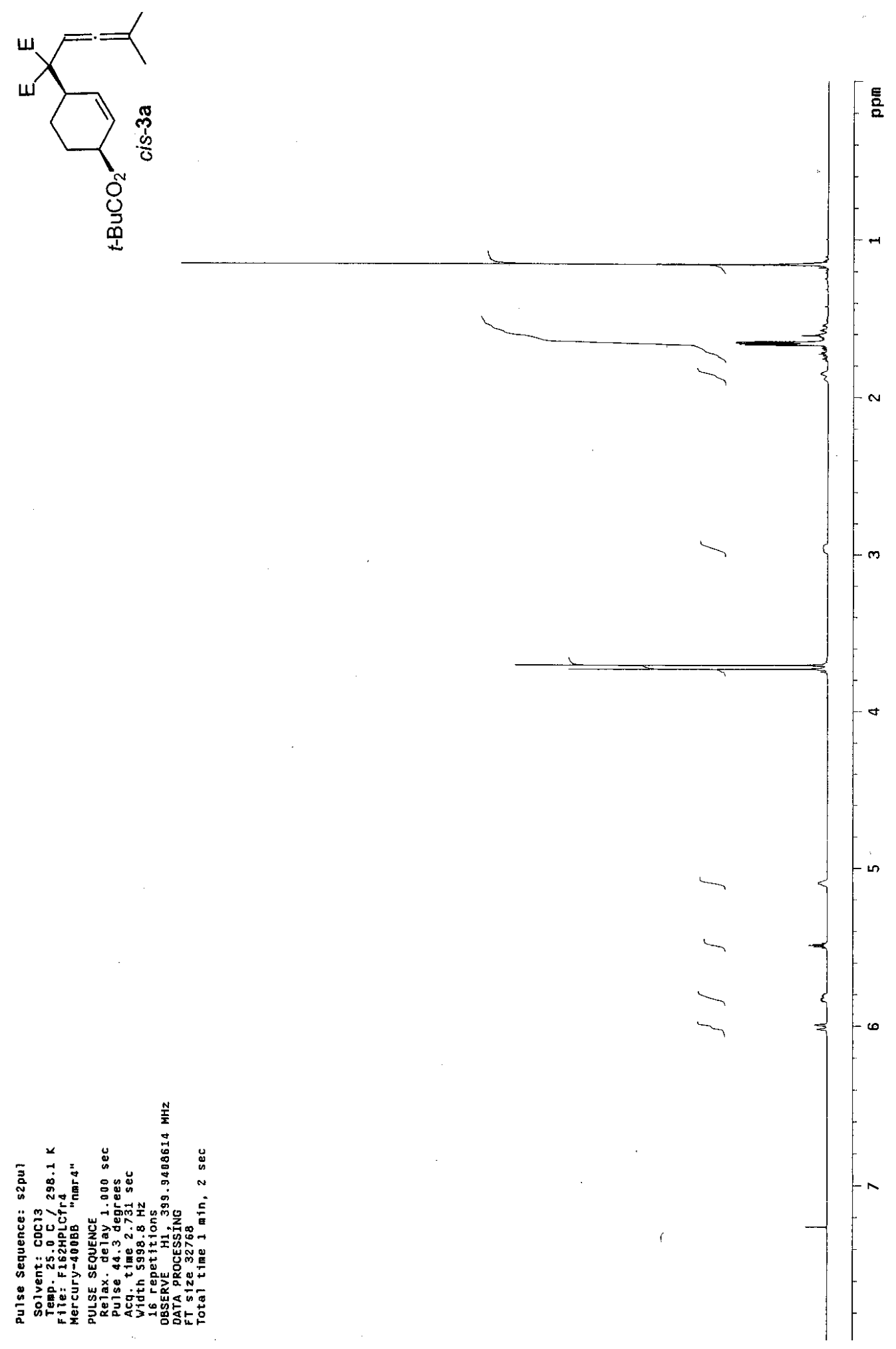



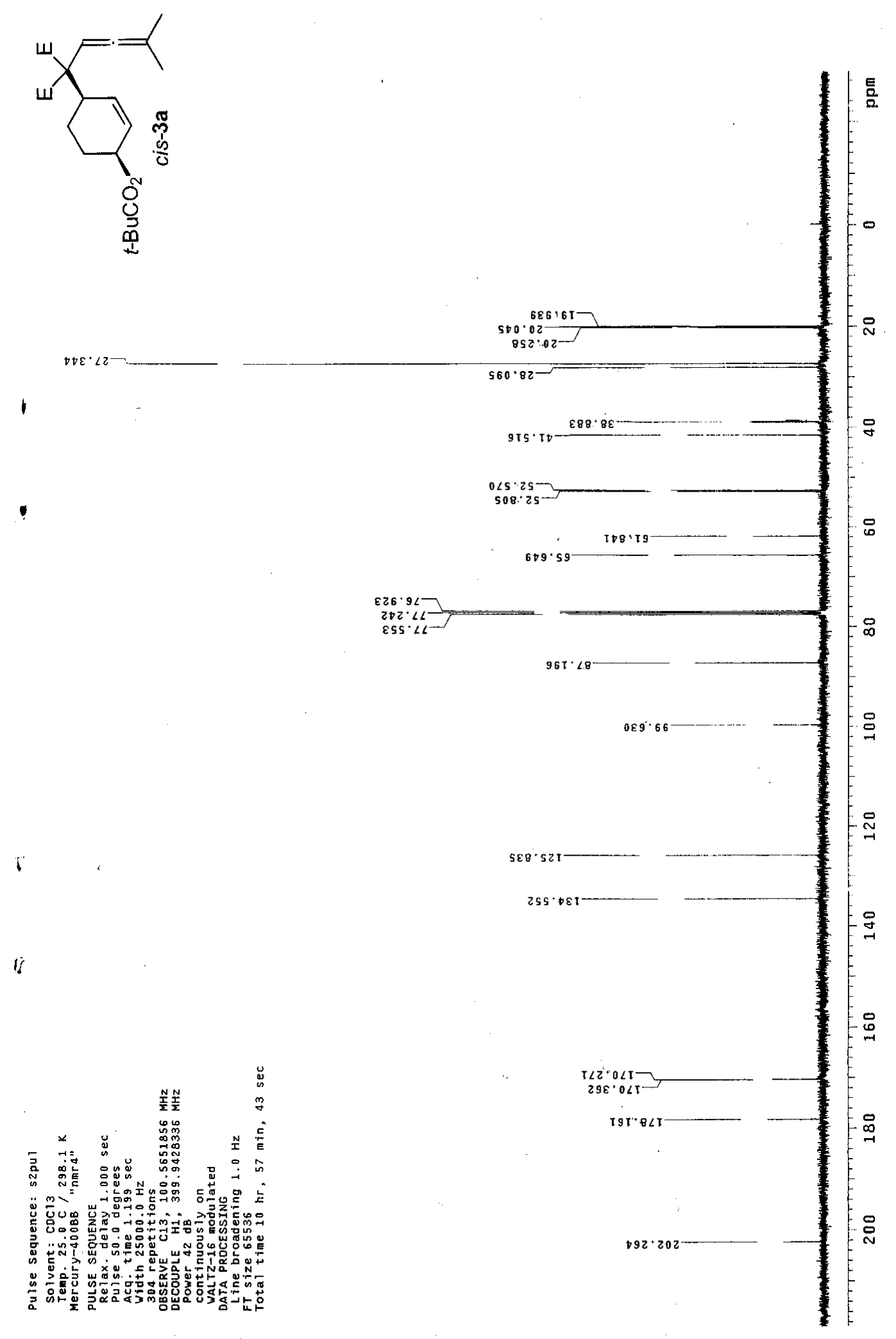

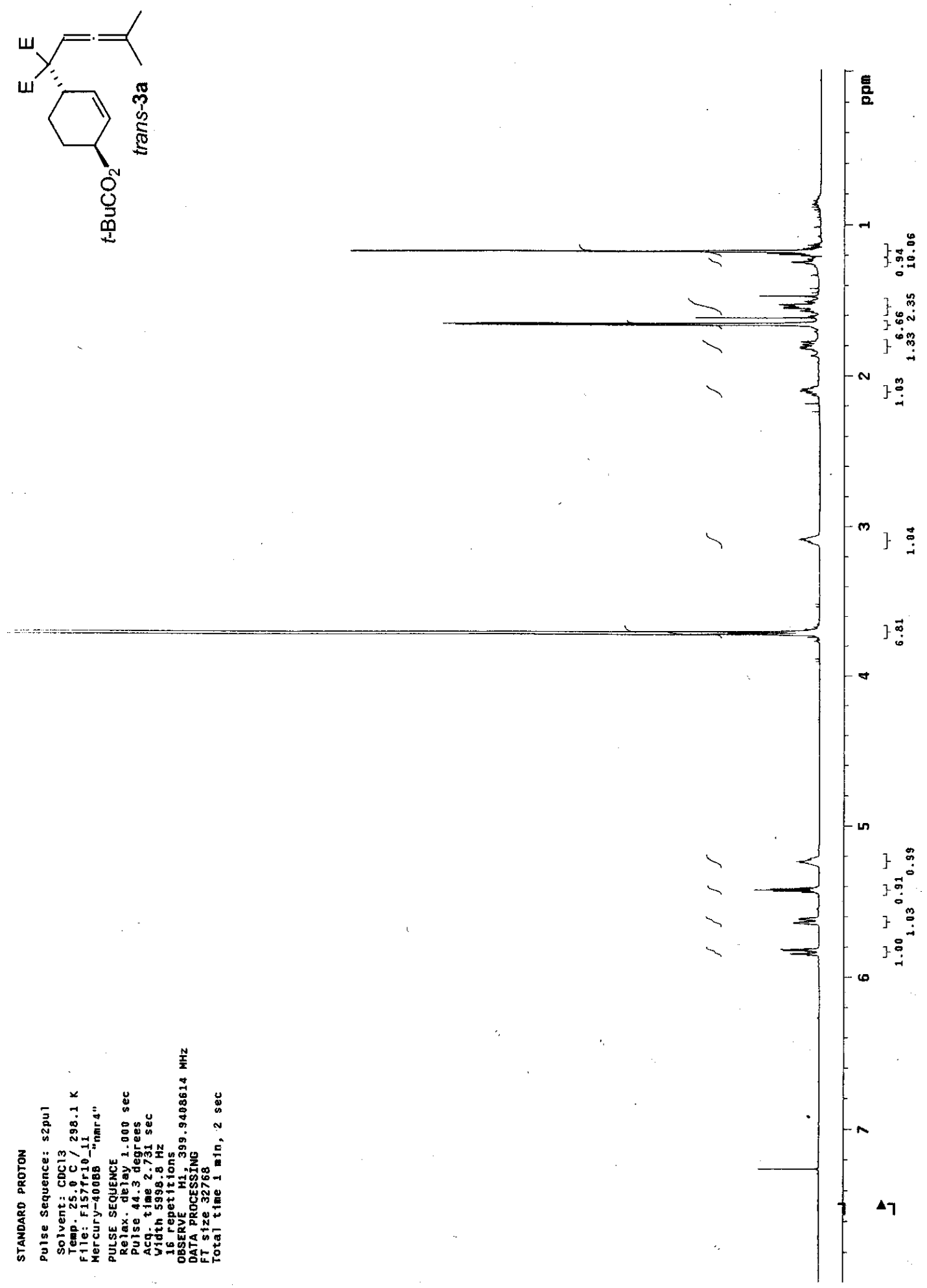

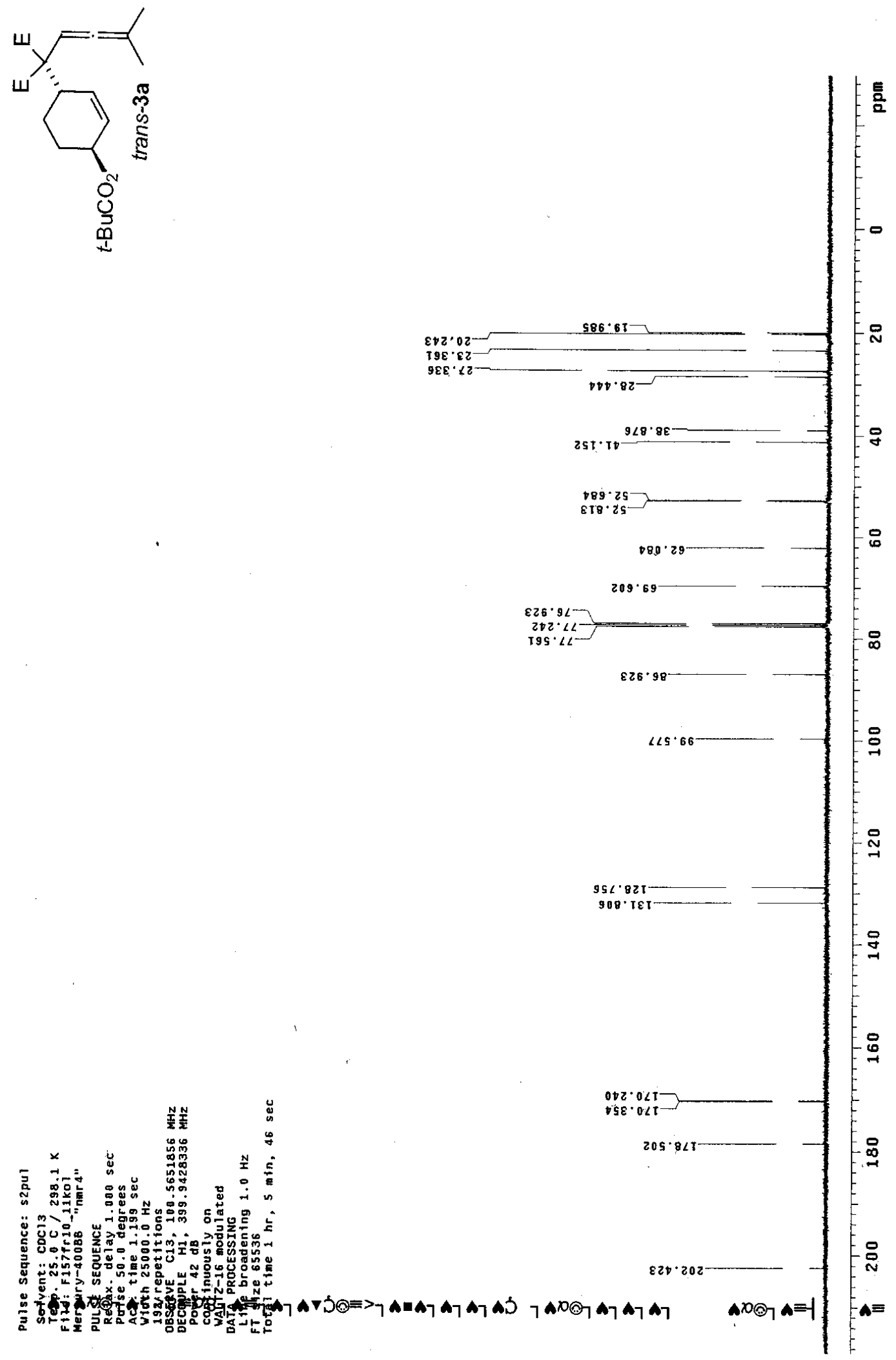


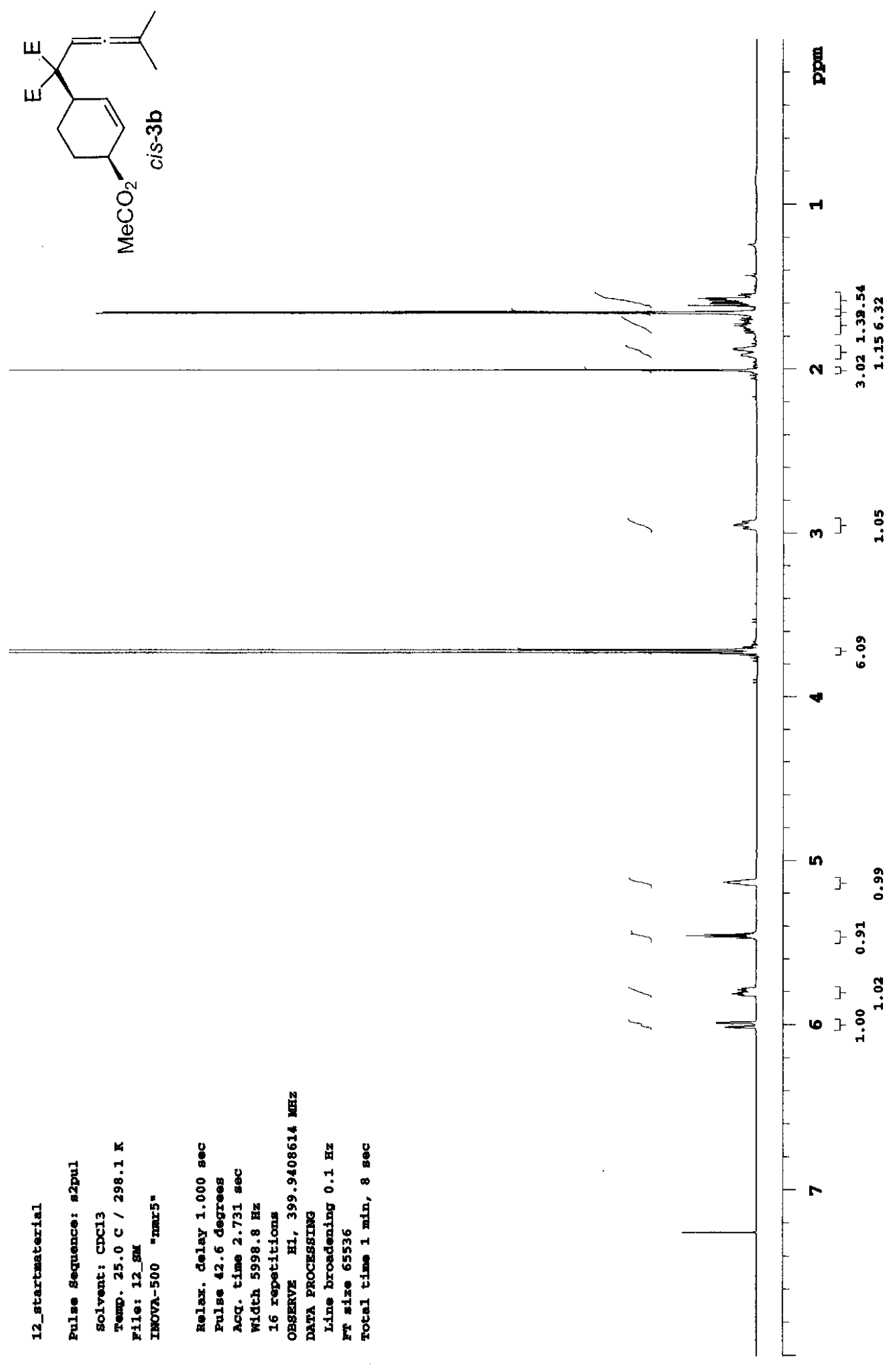



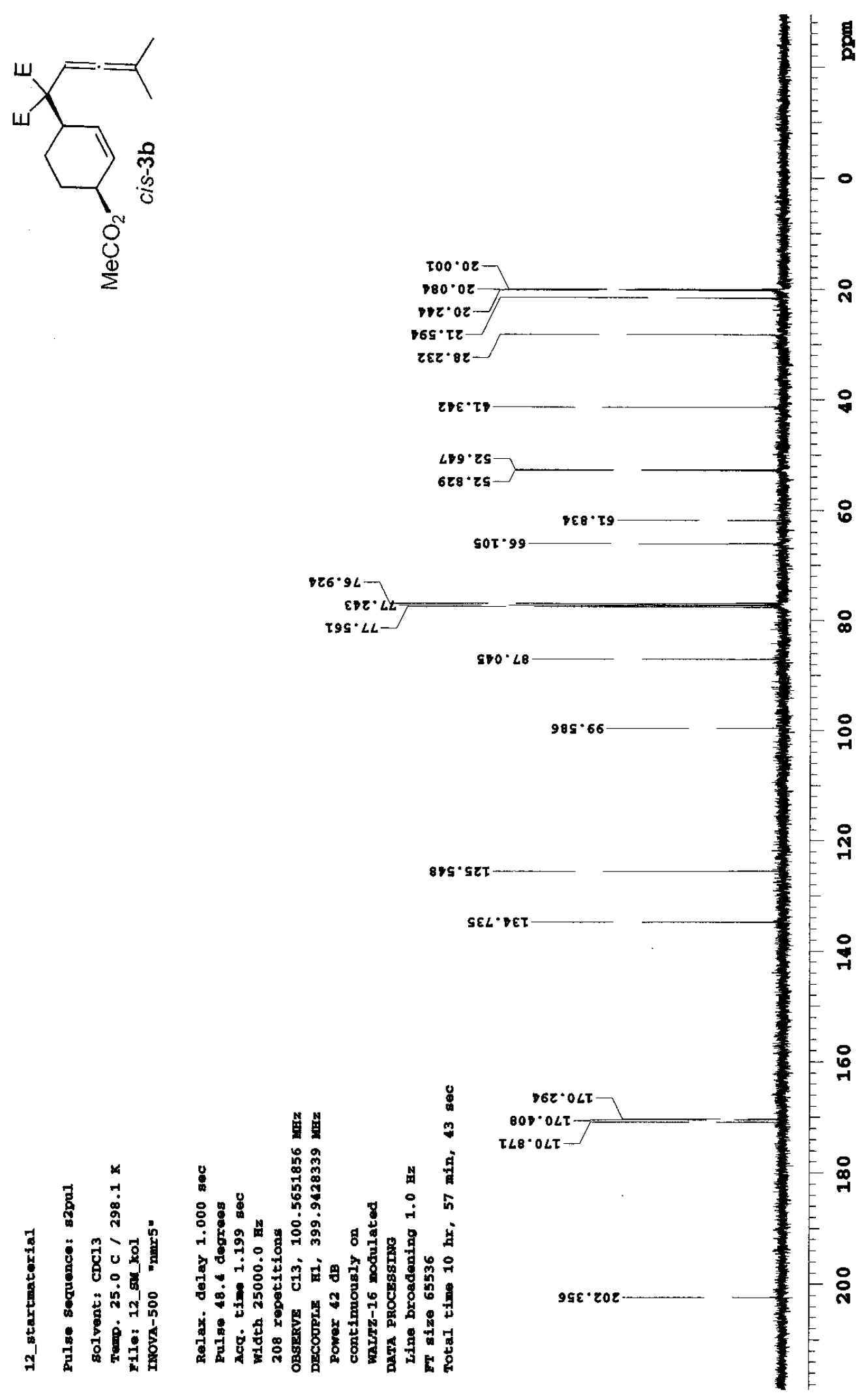

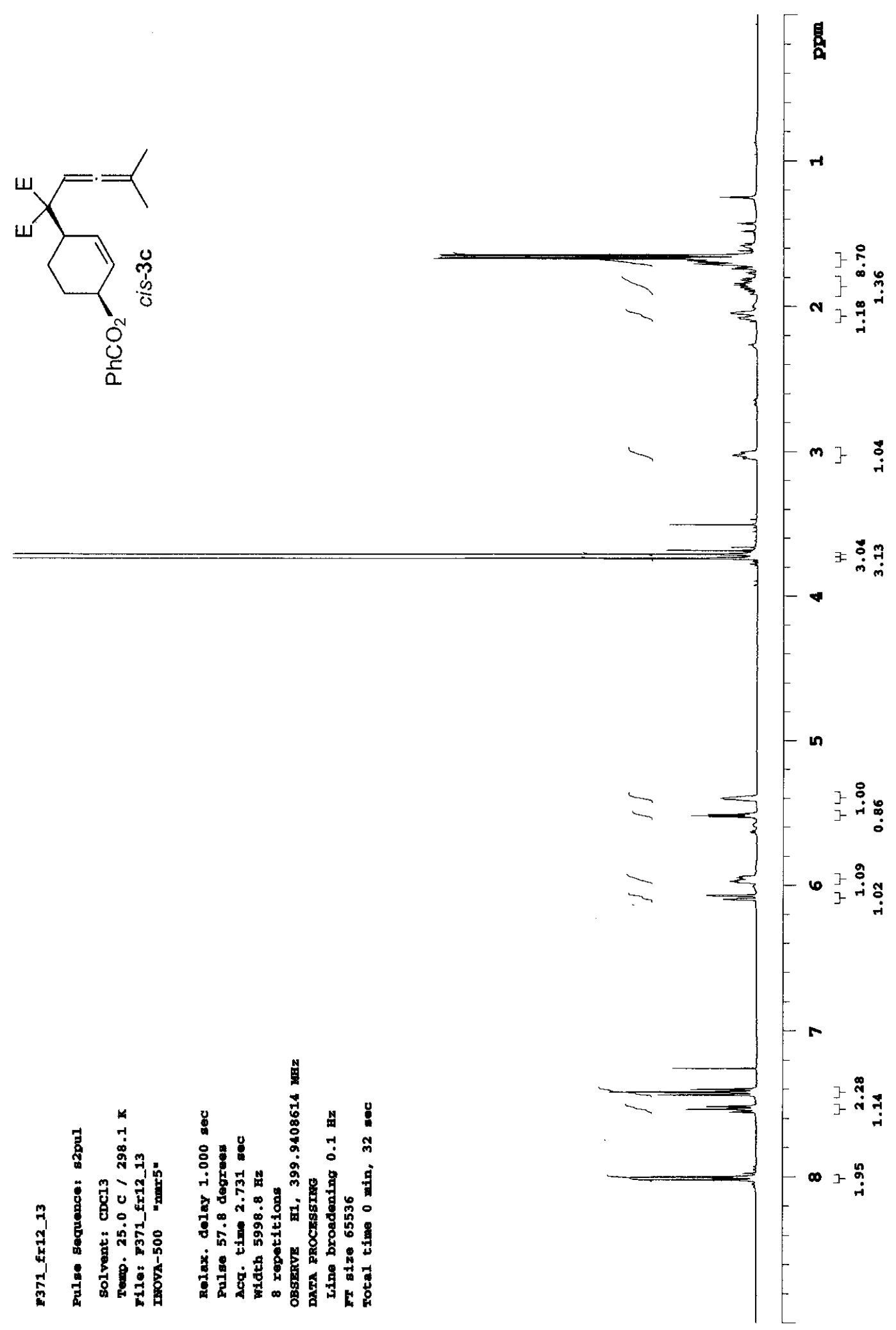

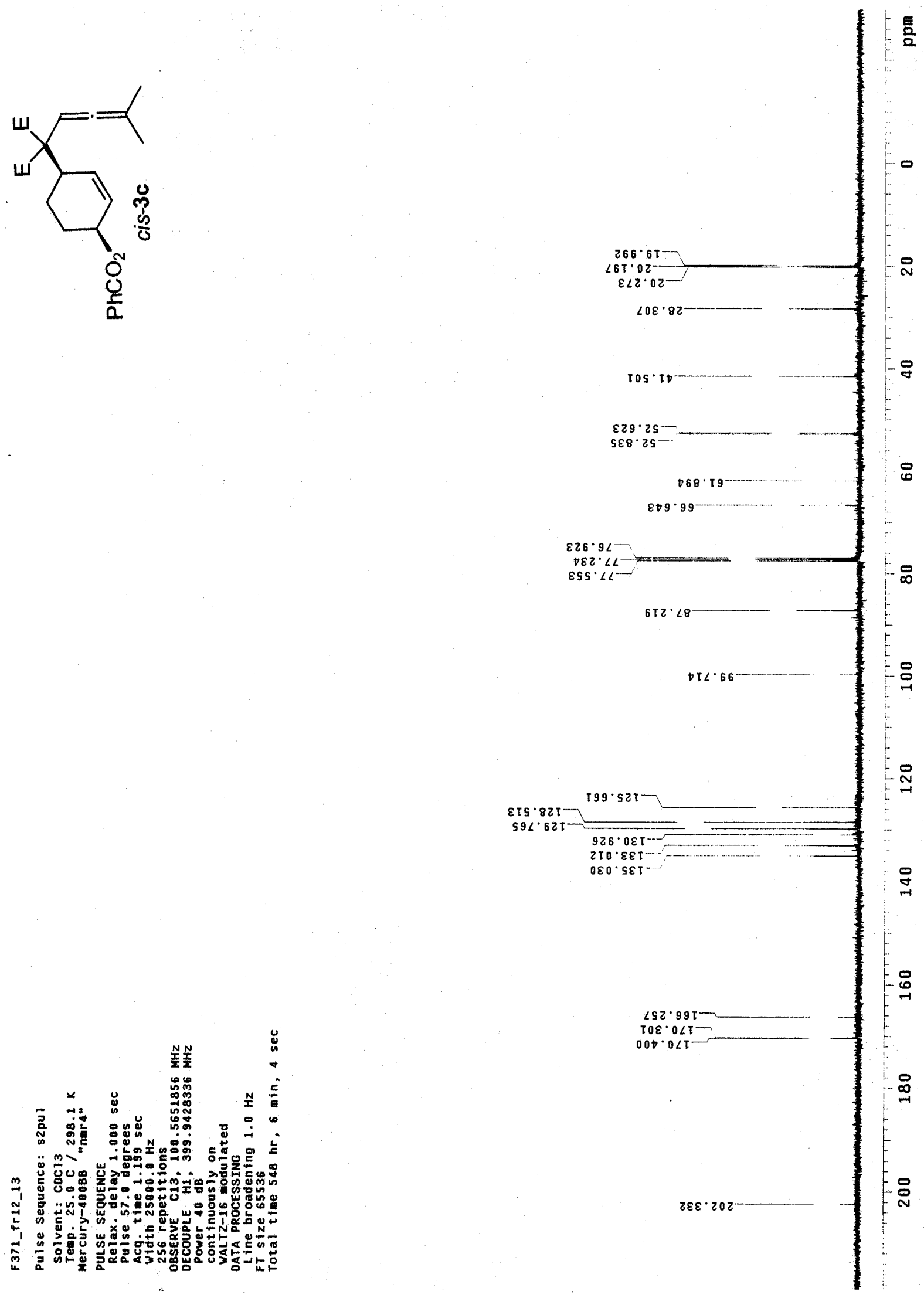

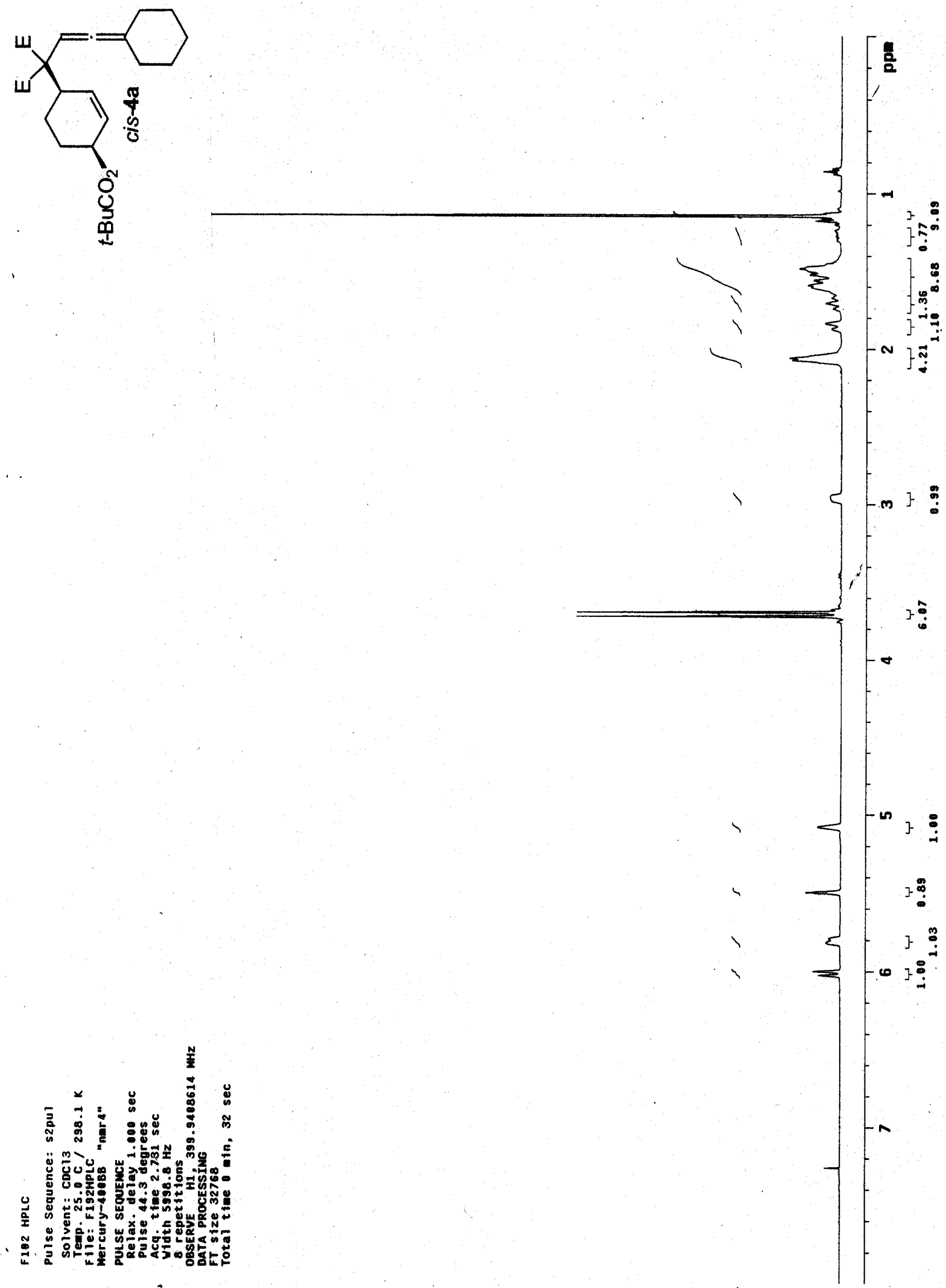

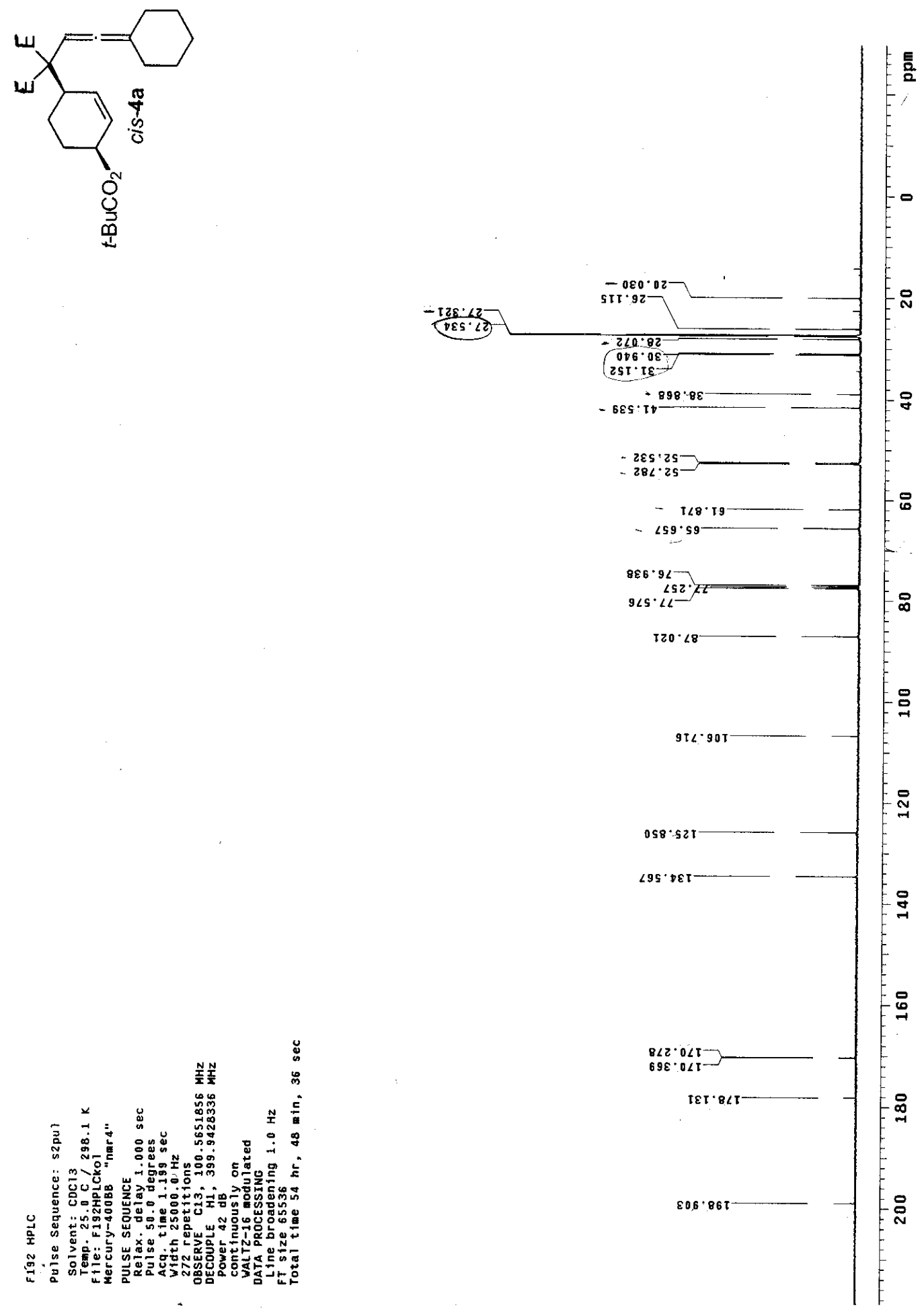


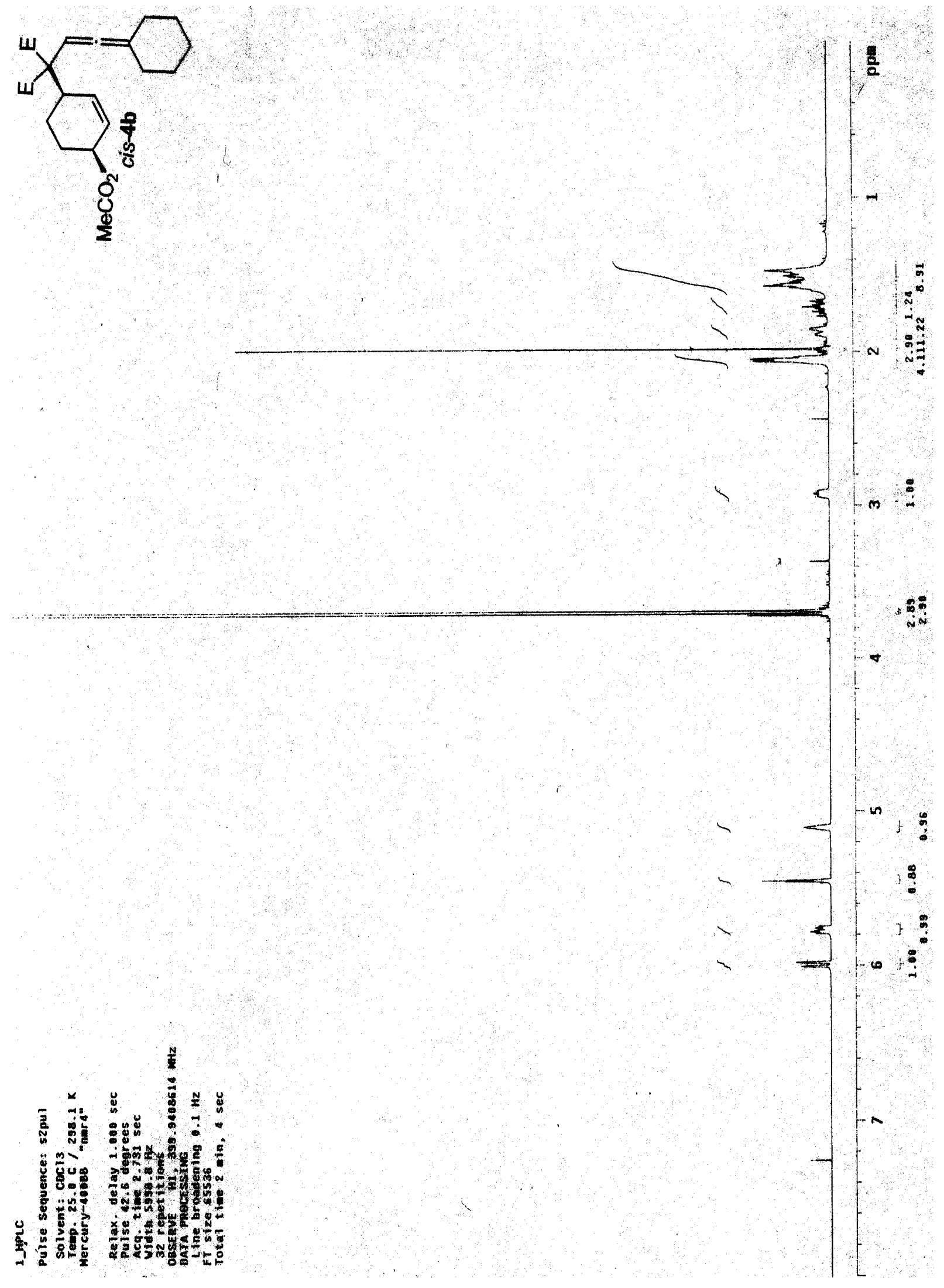



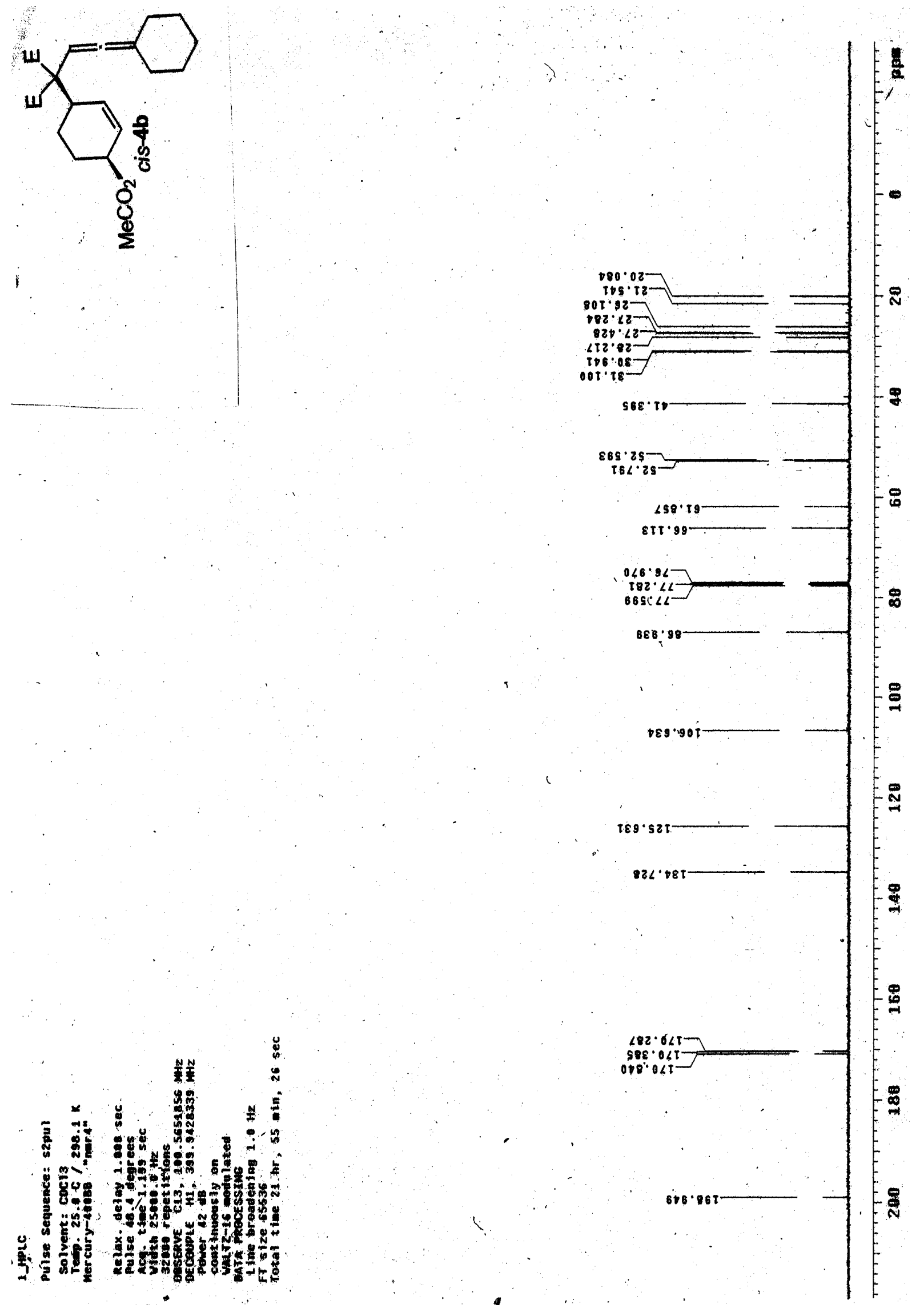


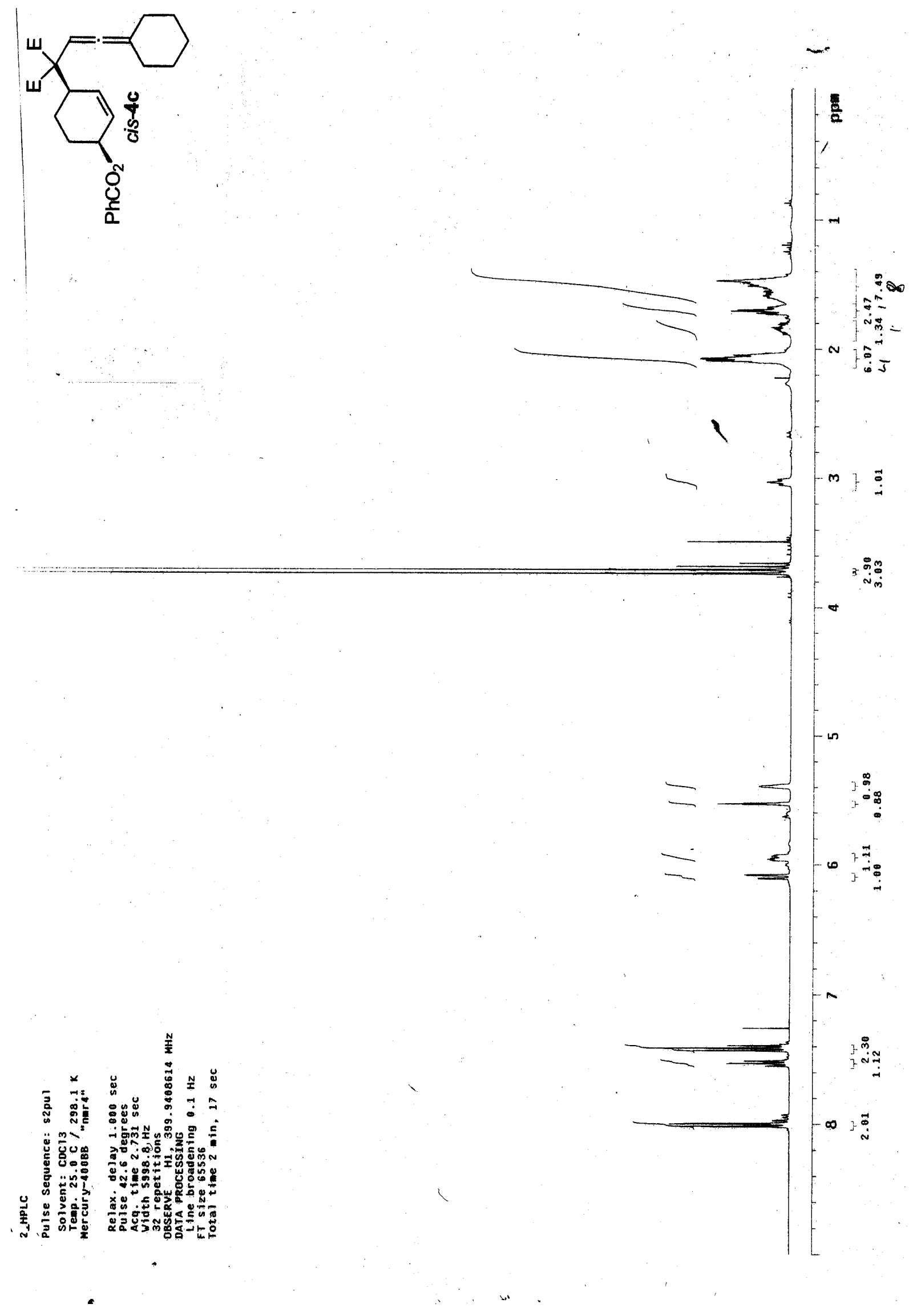



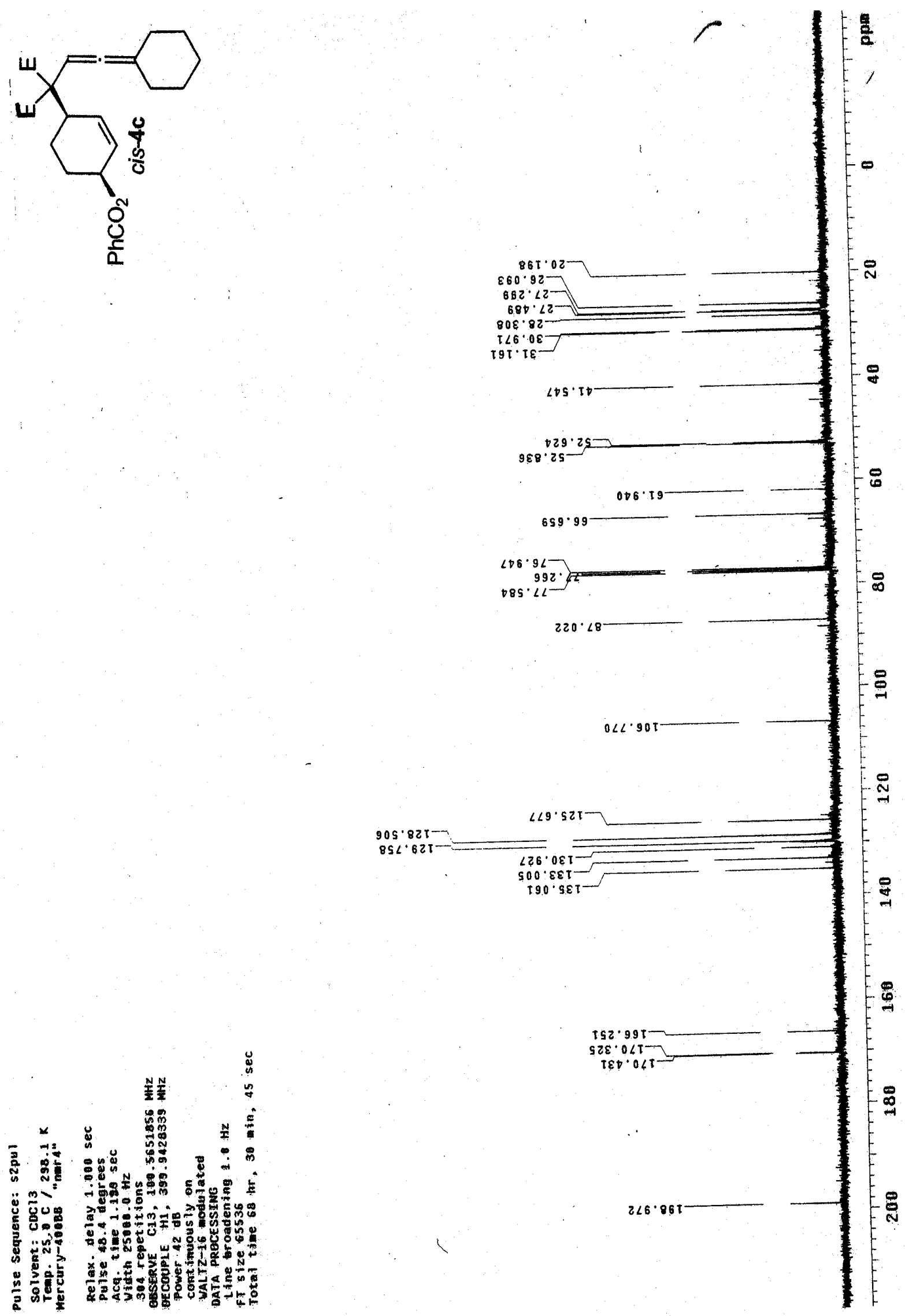


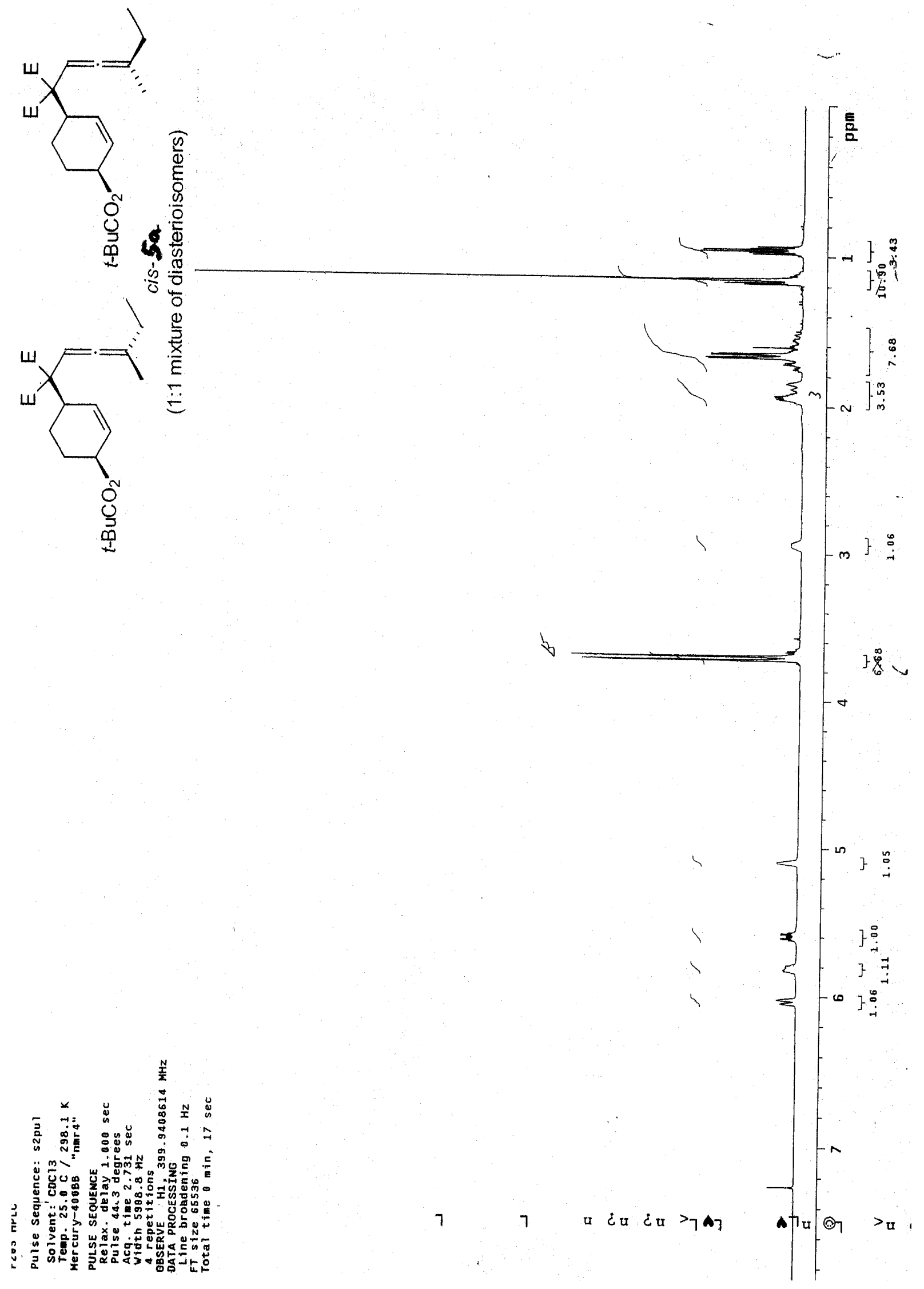



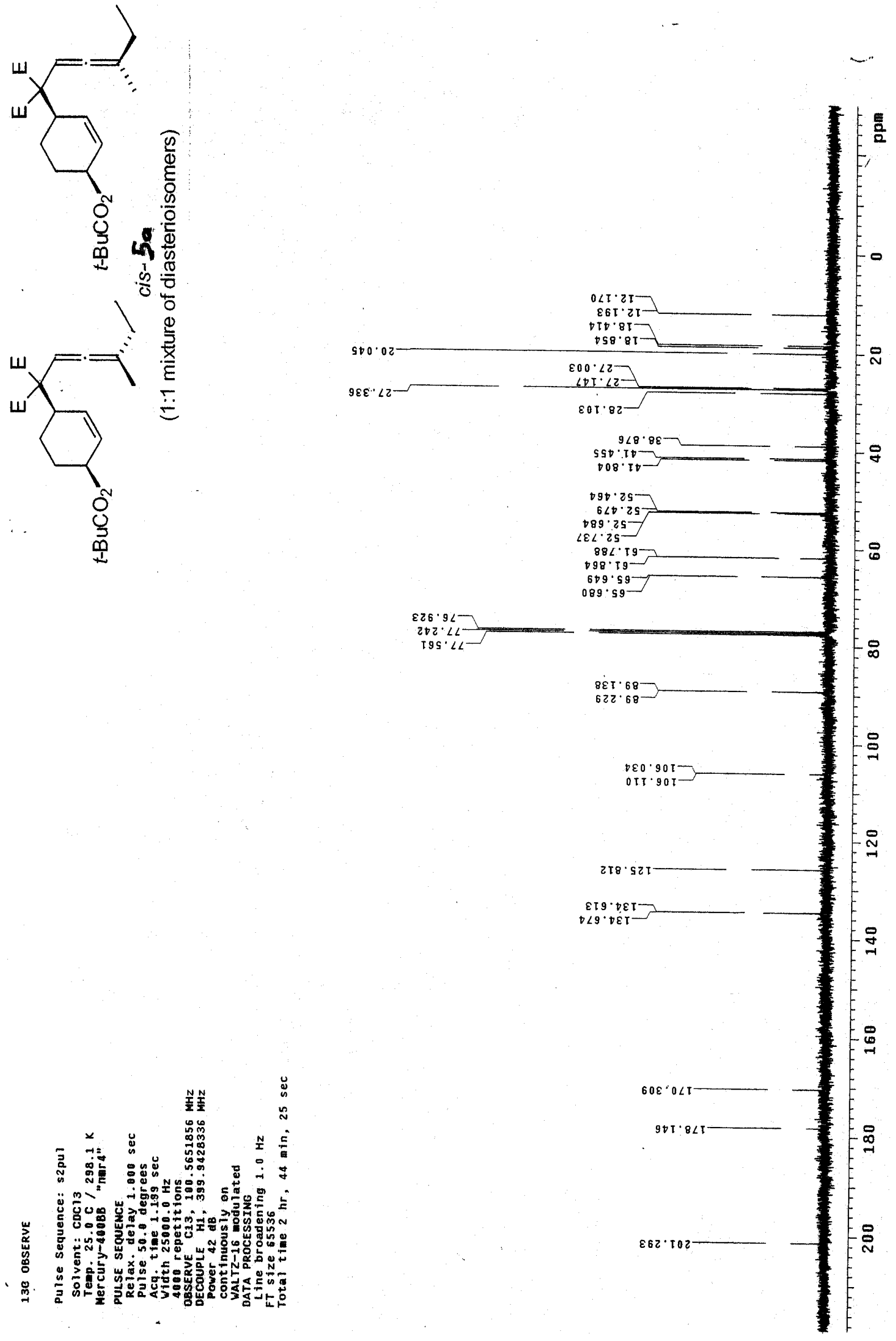

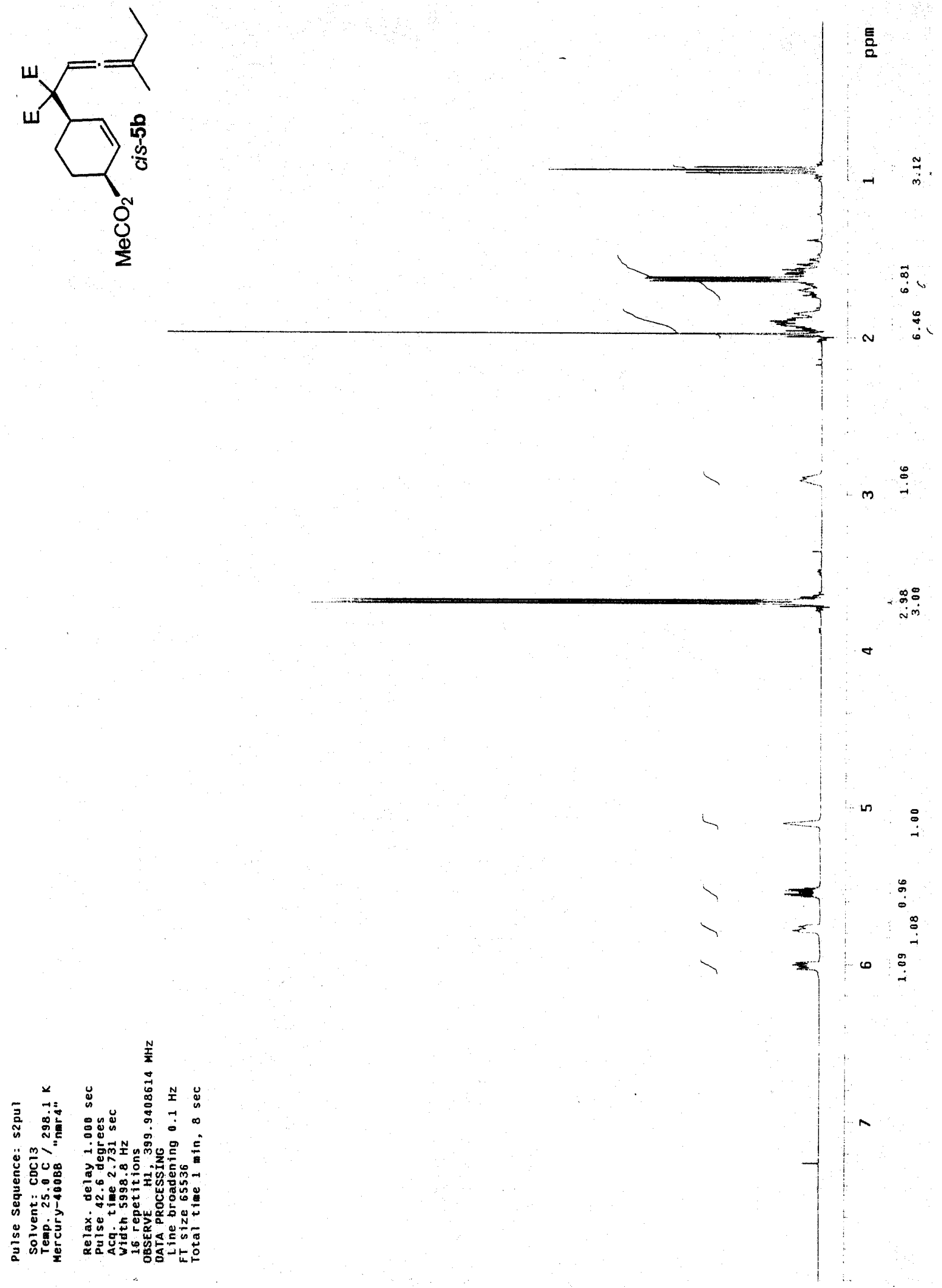

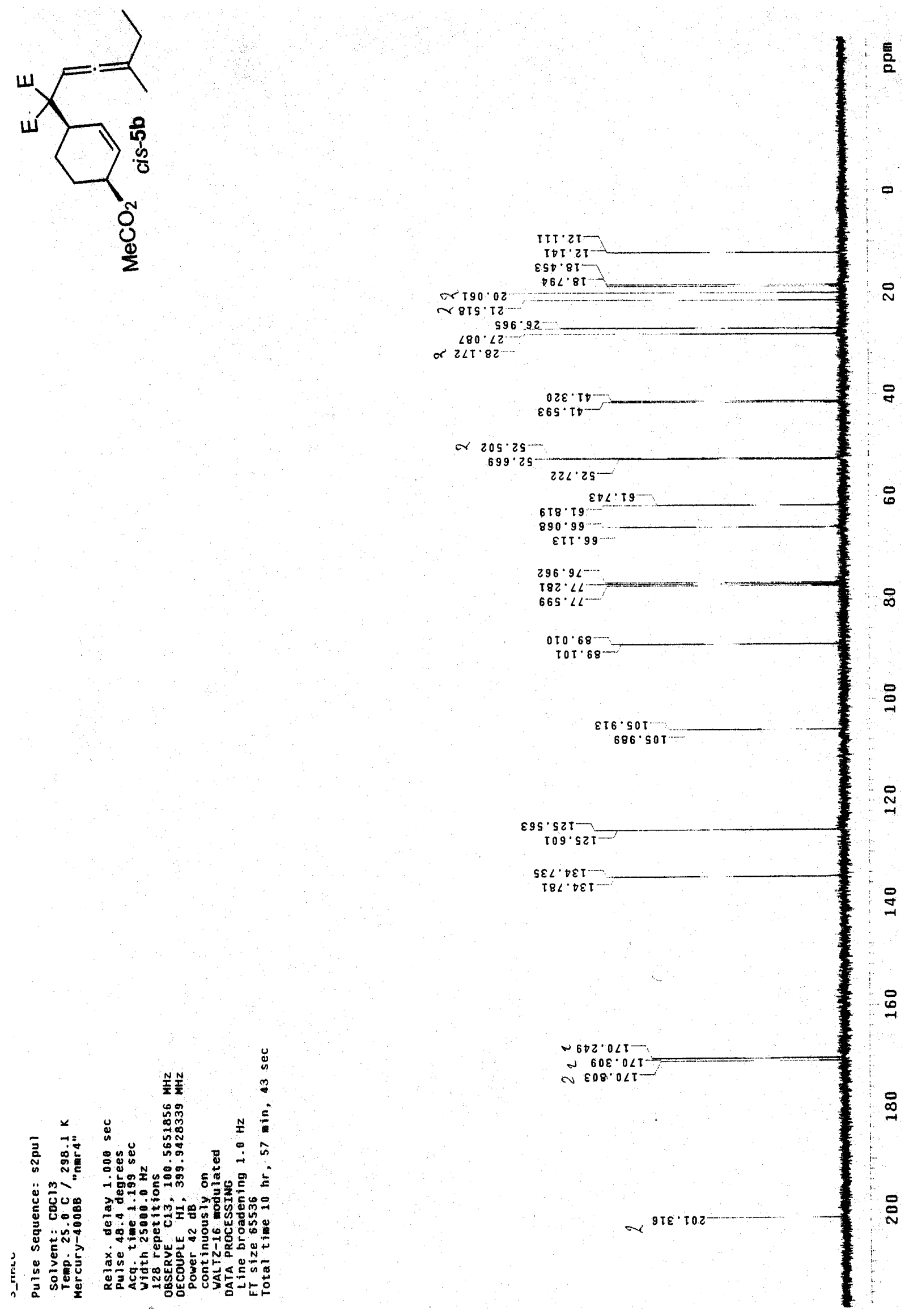

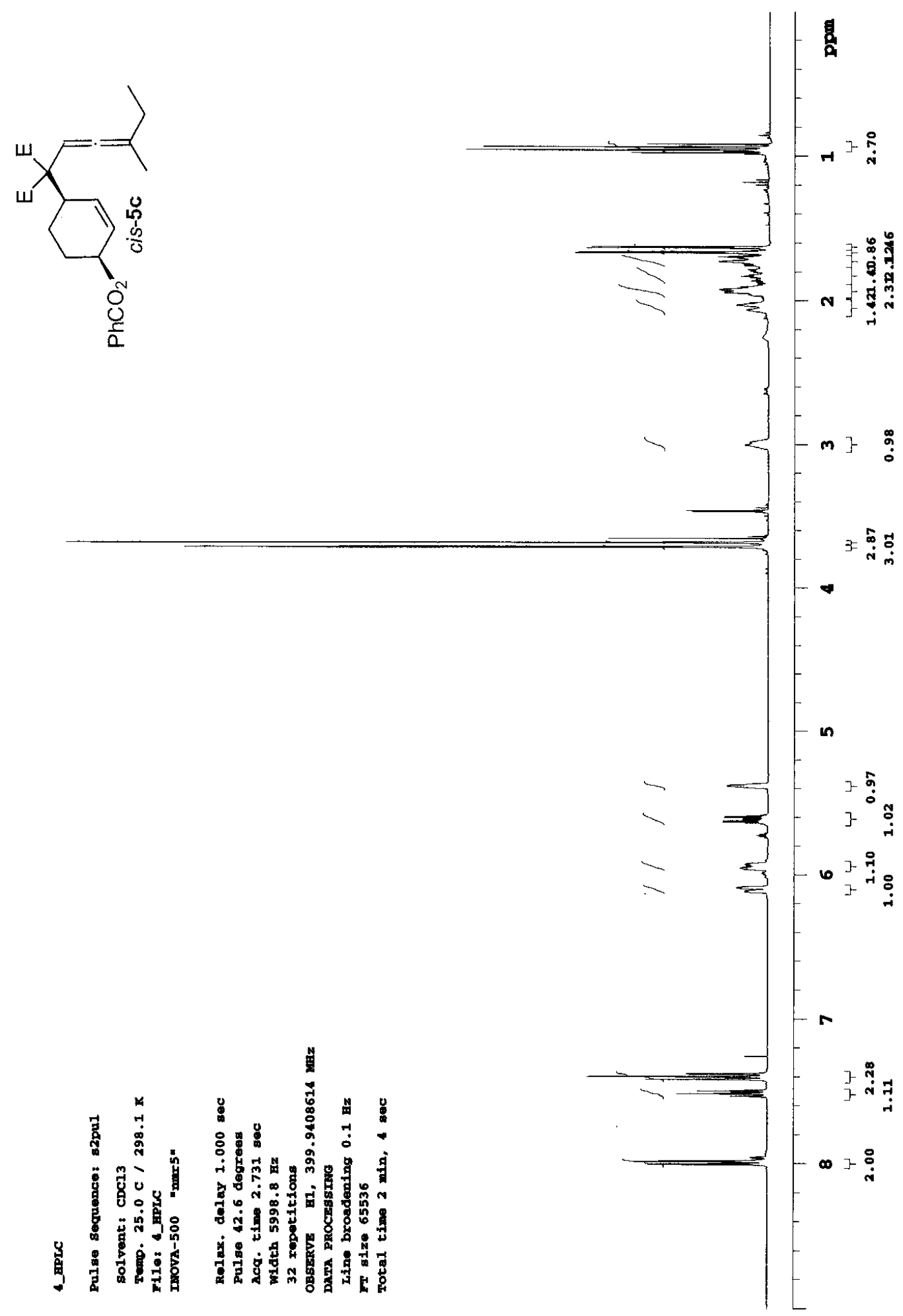

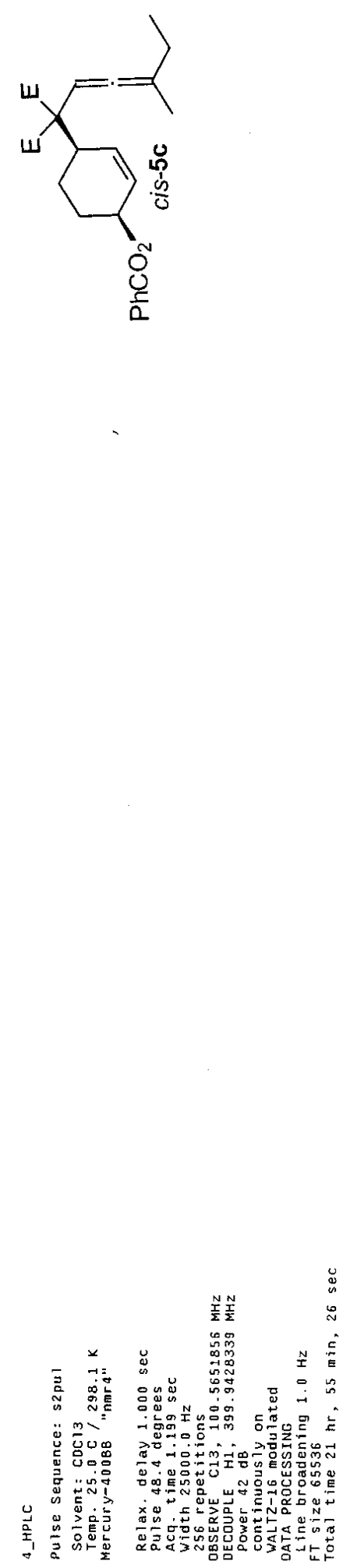

틀

0



요

웅

ळ

$\stackrel{\circ}{\circ}$

$\stackrel{ }{=}$

S $660.82 t$

ov $5 \varepsilon L .62 t$

$g+9 \cdot s<t$

$92 \mathrm{~T} \cdot 90 \mathrm{~T}$

โ $99^{\circ} 5 \mathrm{Z}$ I

$(-\ldots, \ldots \ldots$

or TTG. $0 \varepsilon T$

$866 \cdot 2 \varepsilon \mathrm{I} \cdot \cdots$
$850 \cdot \varepsilon \varepsilon \mathrm{I}$
$\angle \angle 0 \cdot 5 \varepsilon \mathrm{T}$

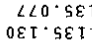

$\underset{-}{\stackrel{9}{7}}$

$\underset{0}{0}$

$\begin{array}{r}502 \cdot 99 \mathrm{~T} \\ \quad 992.04 \mathrm{~T} \\ \hline\end{array}$

$\angle T E \cdot 0 \angle T$

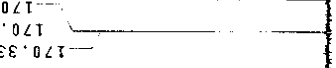

$\underset{-1}{\infty}$

$\therefore \nabla 5 \varepsilon^{\circ}+02+\ldots$ 


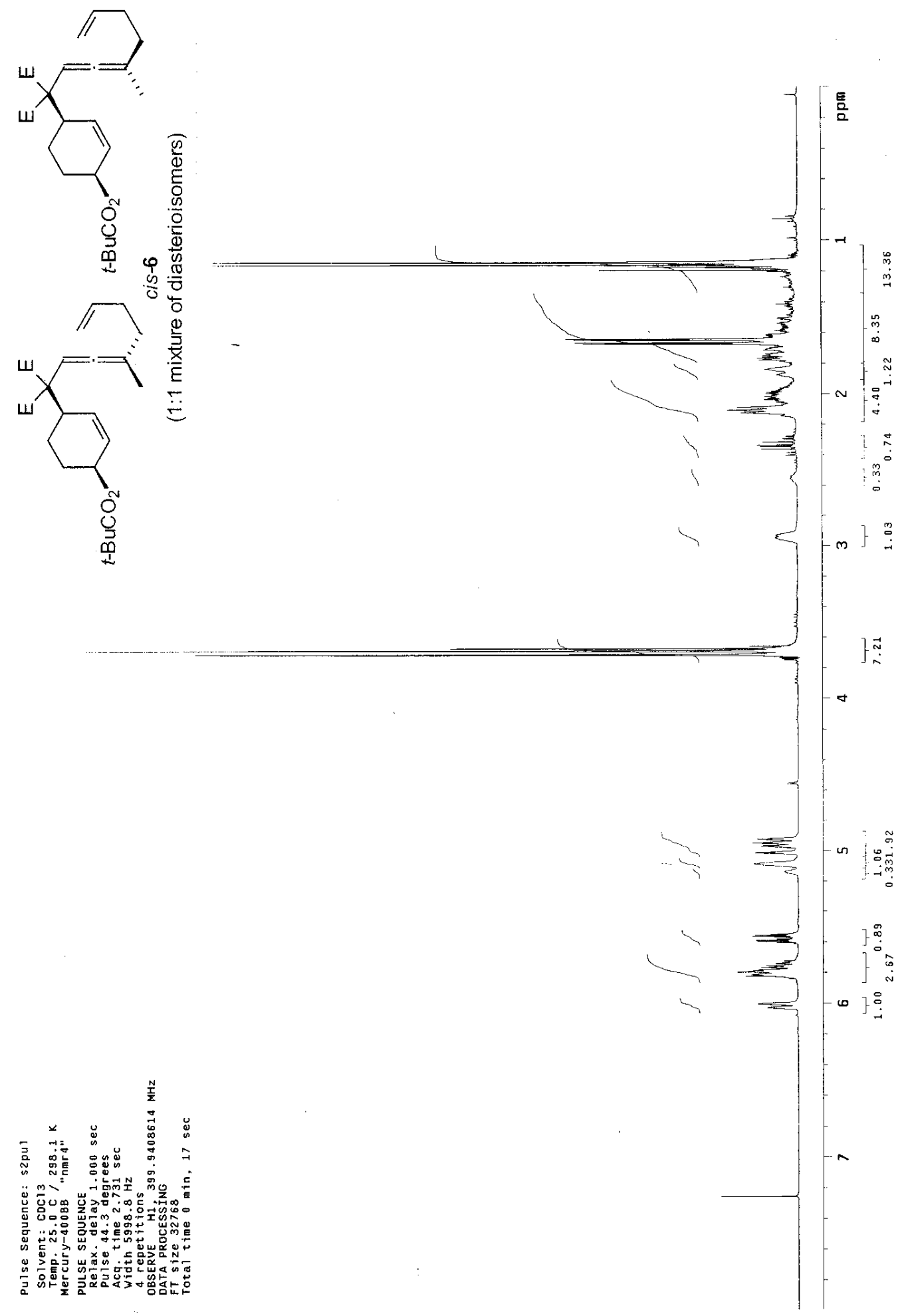



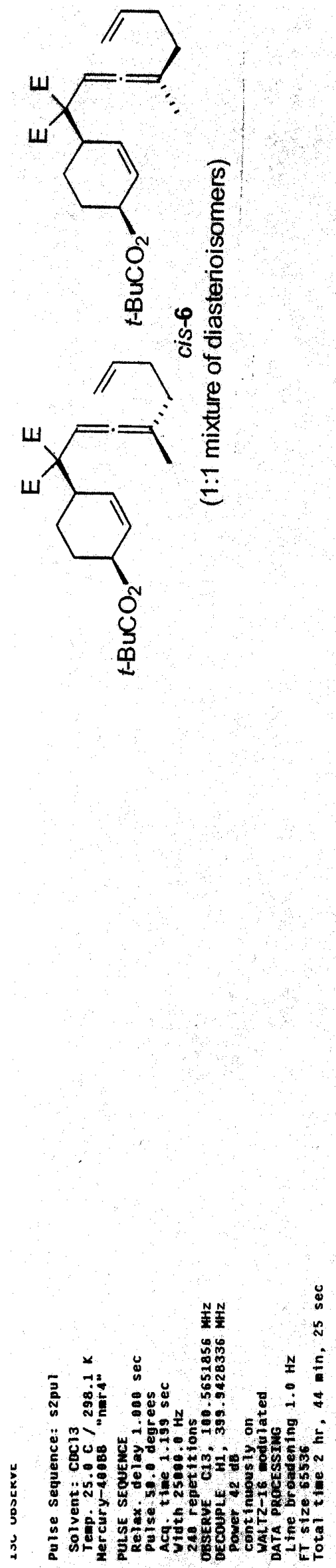

$9 \varepsilon \varepsilon \cdot \angle 2-$

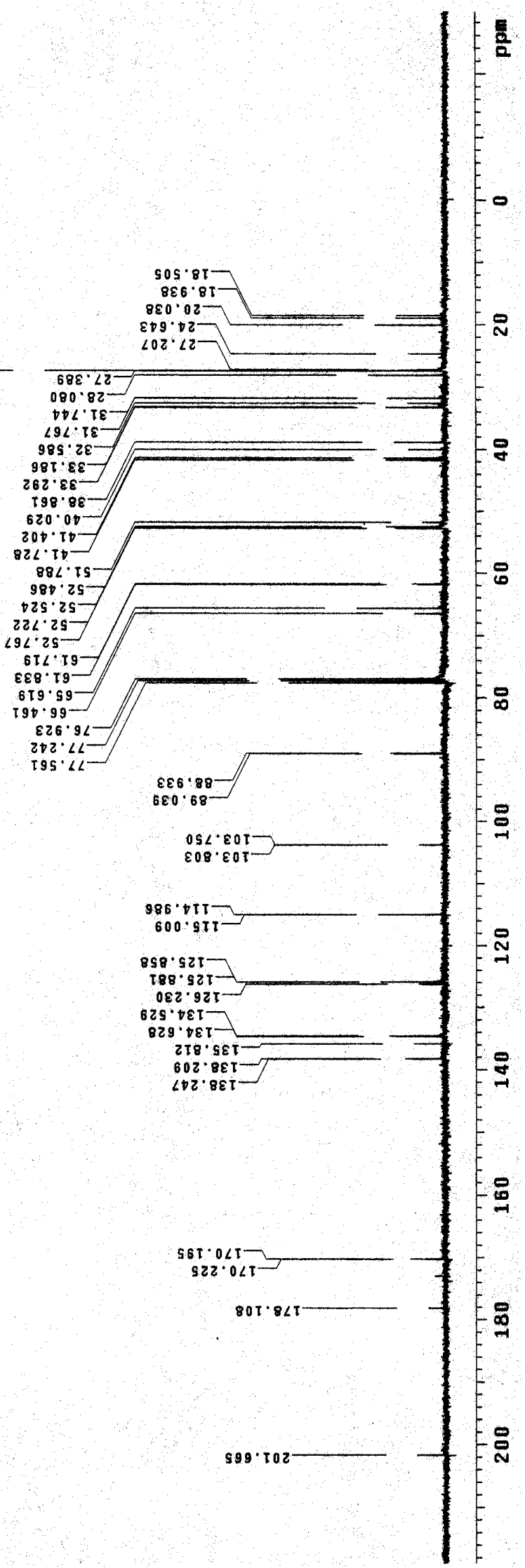




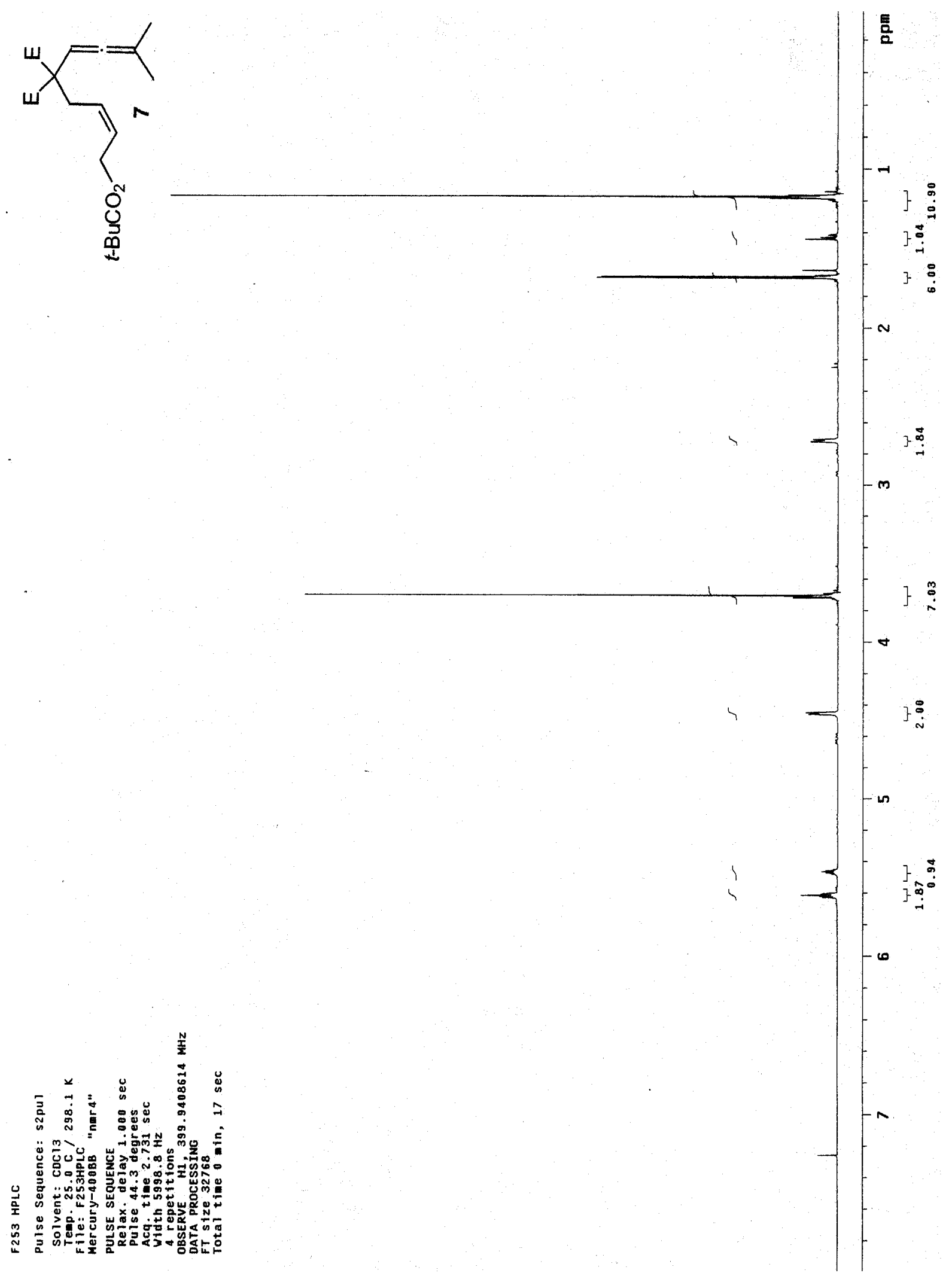



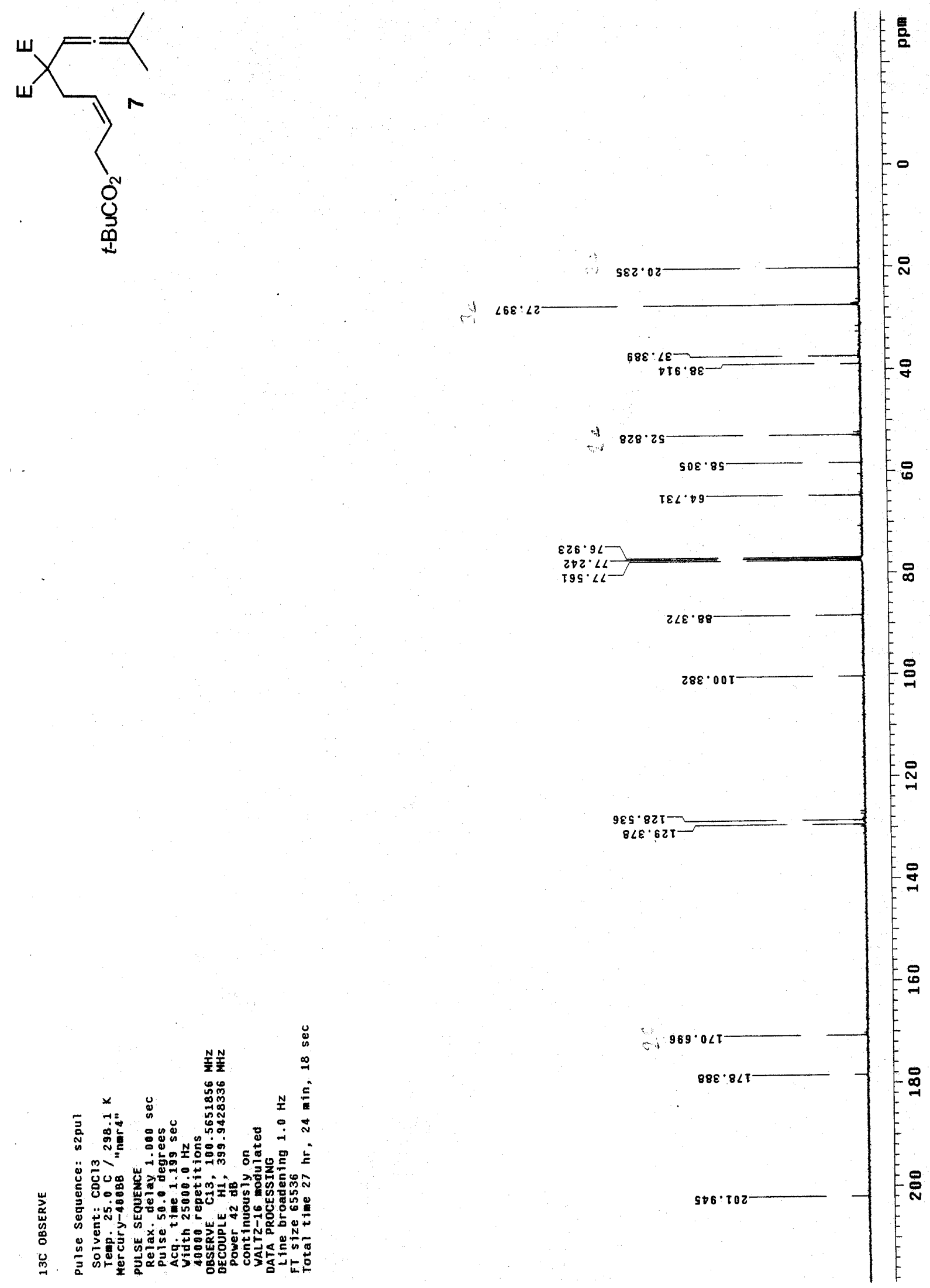


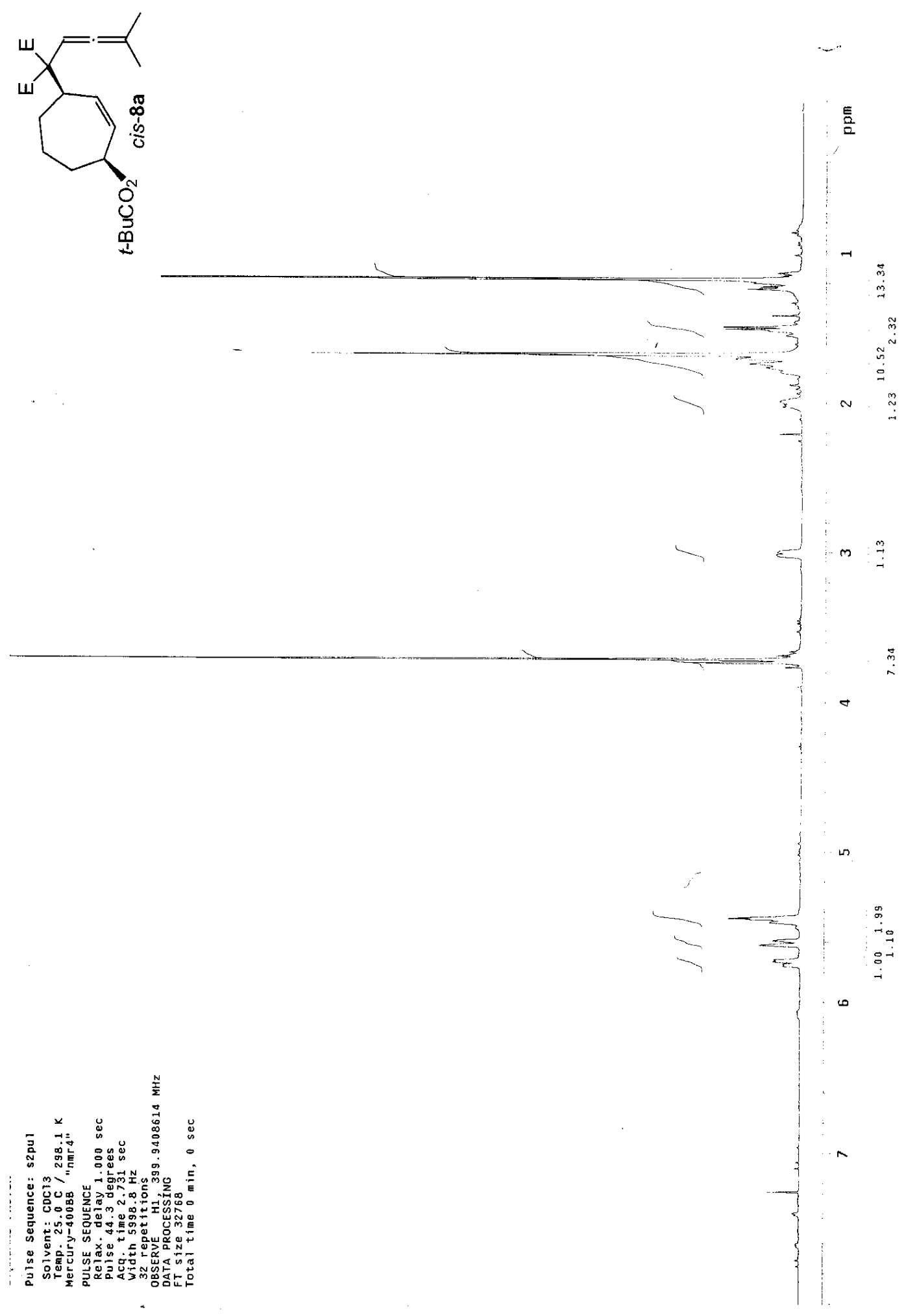



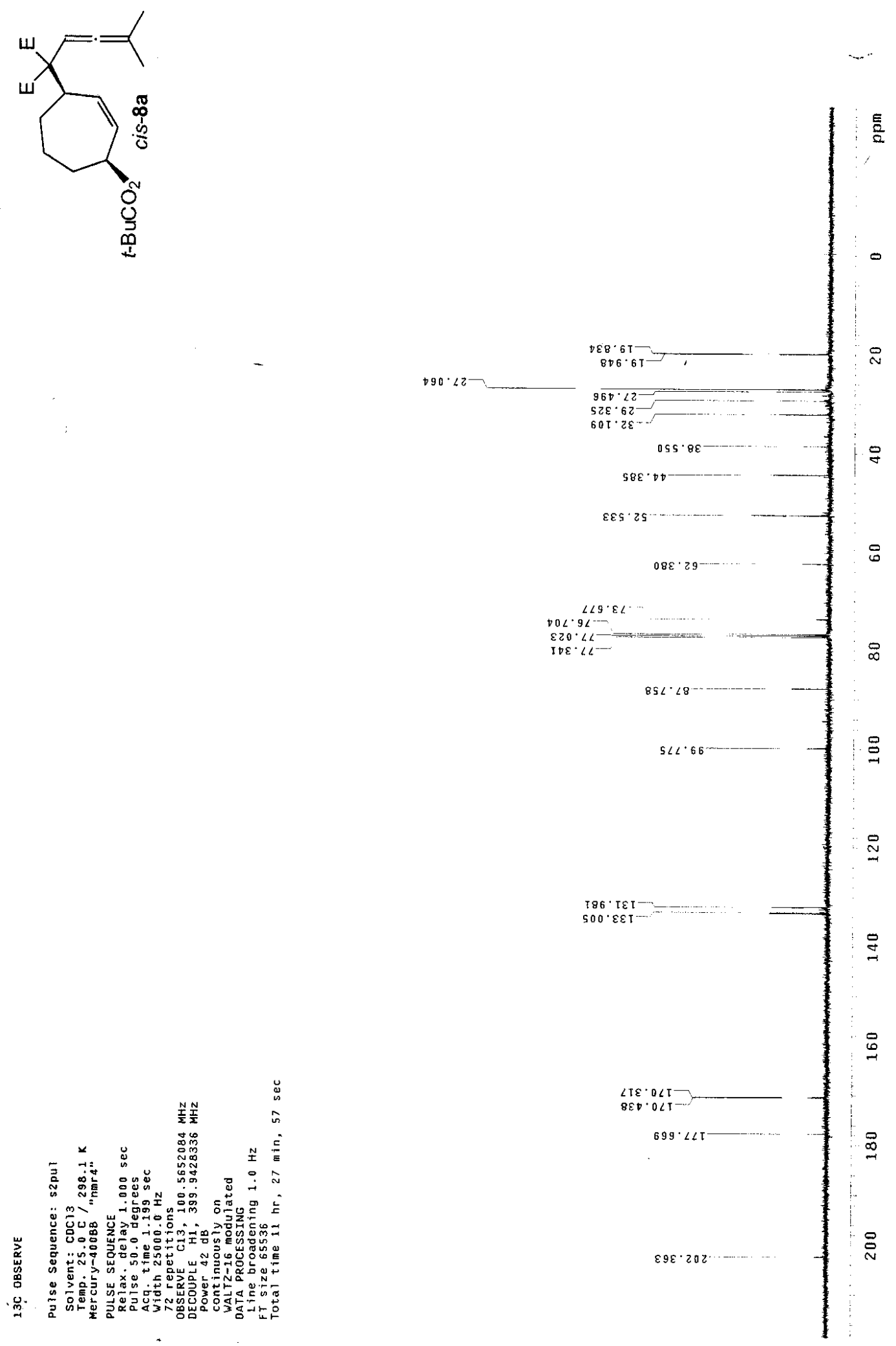


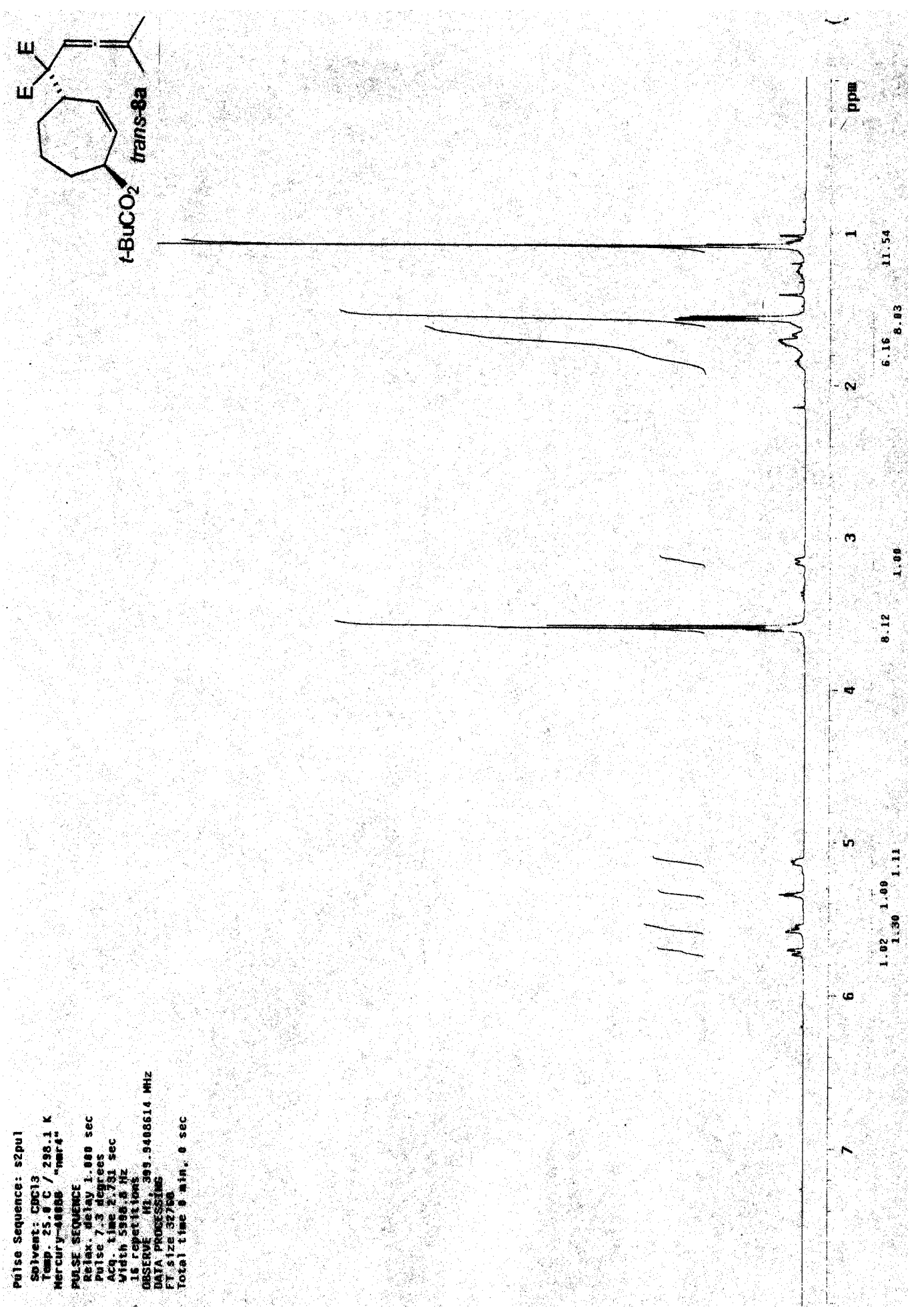




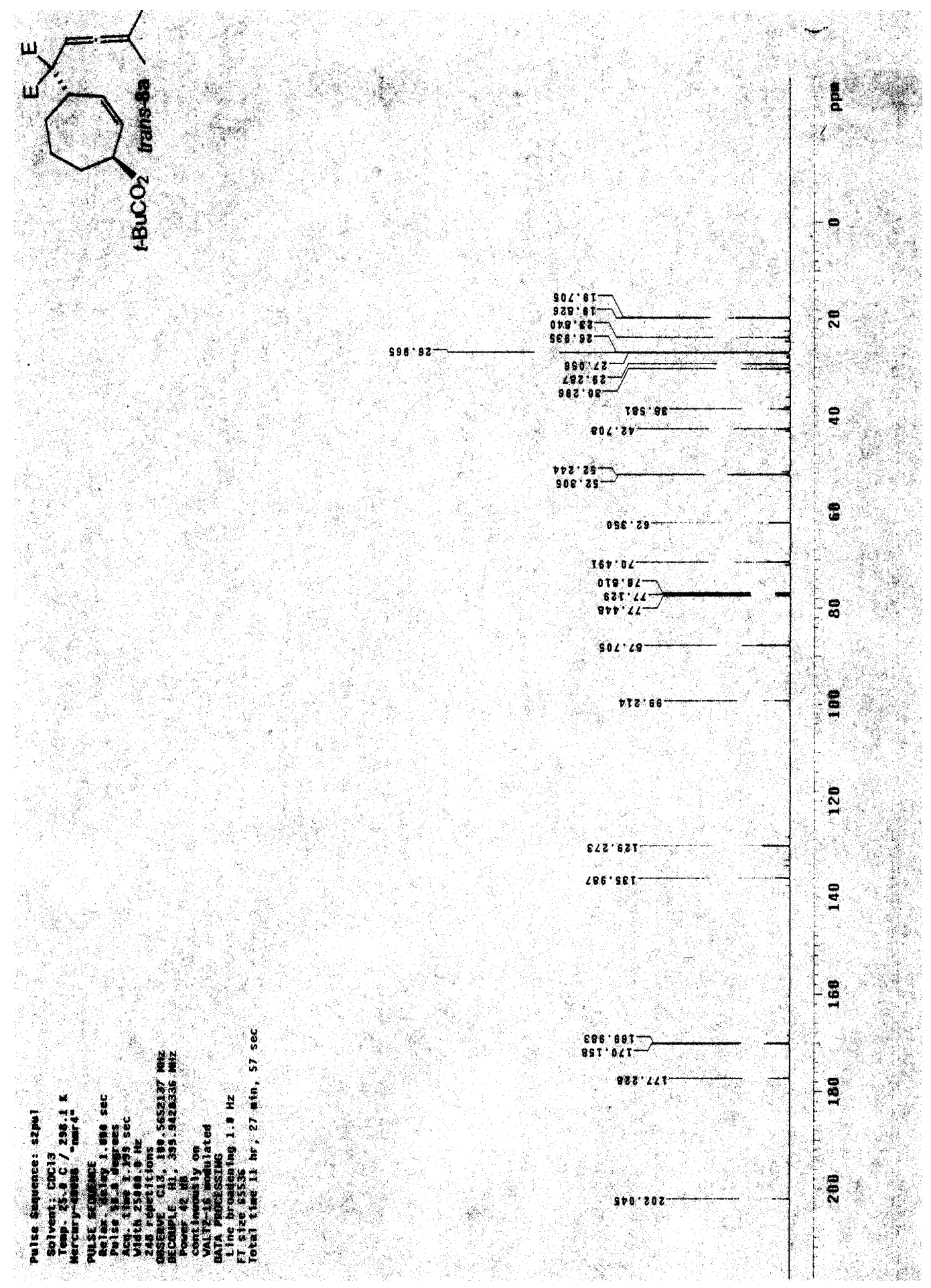




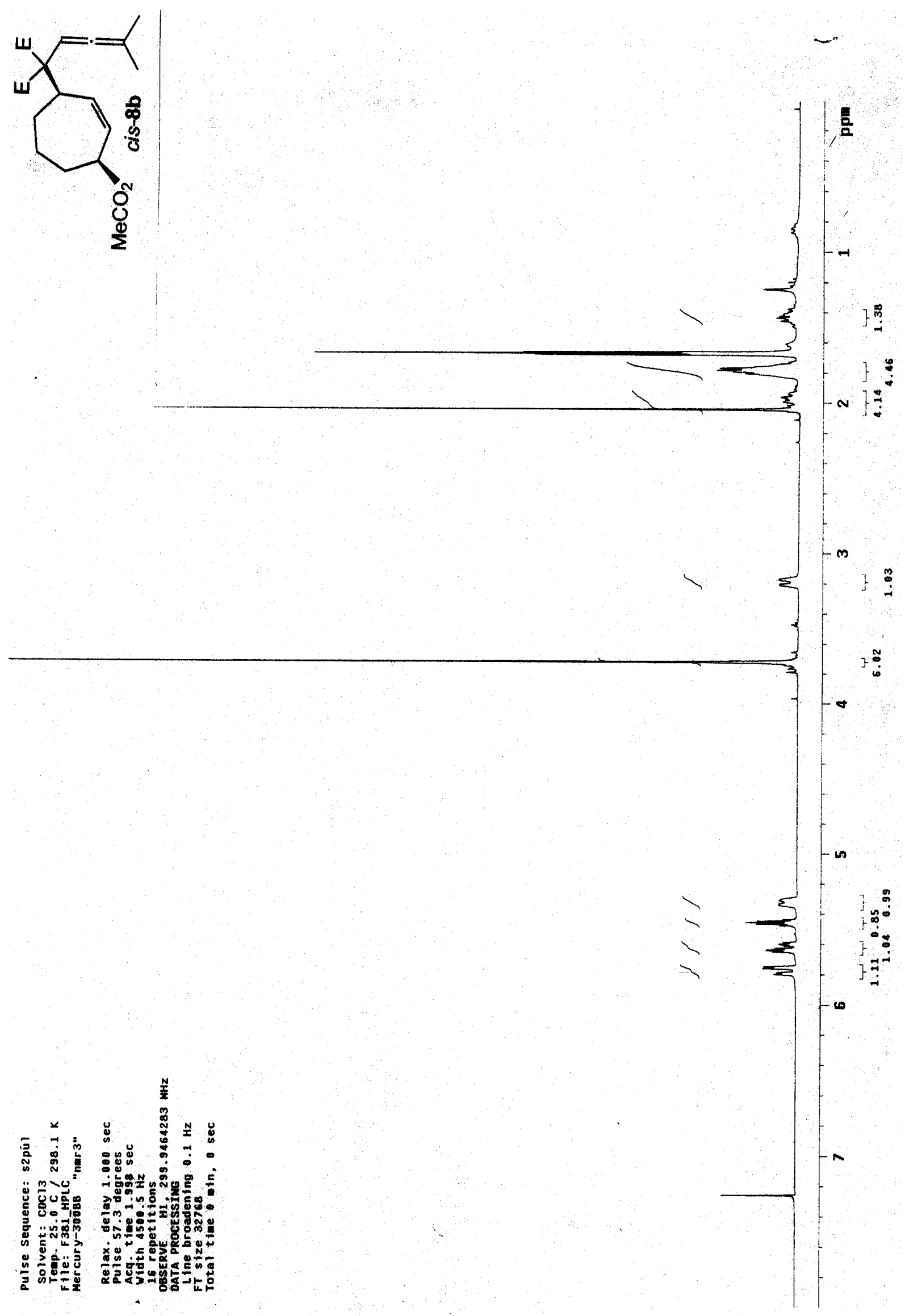



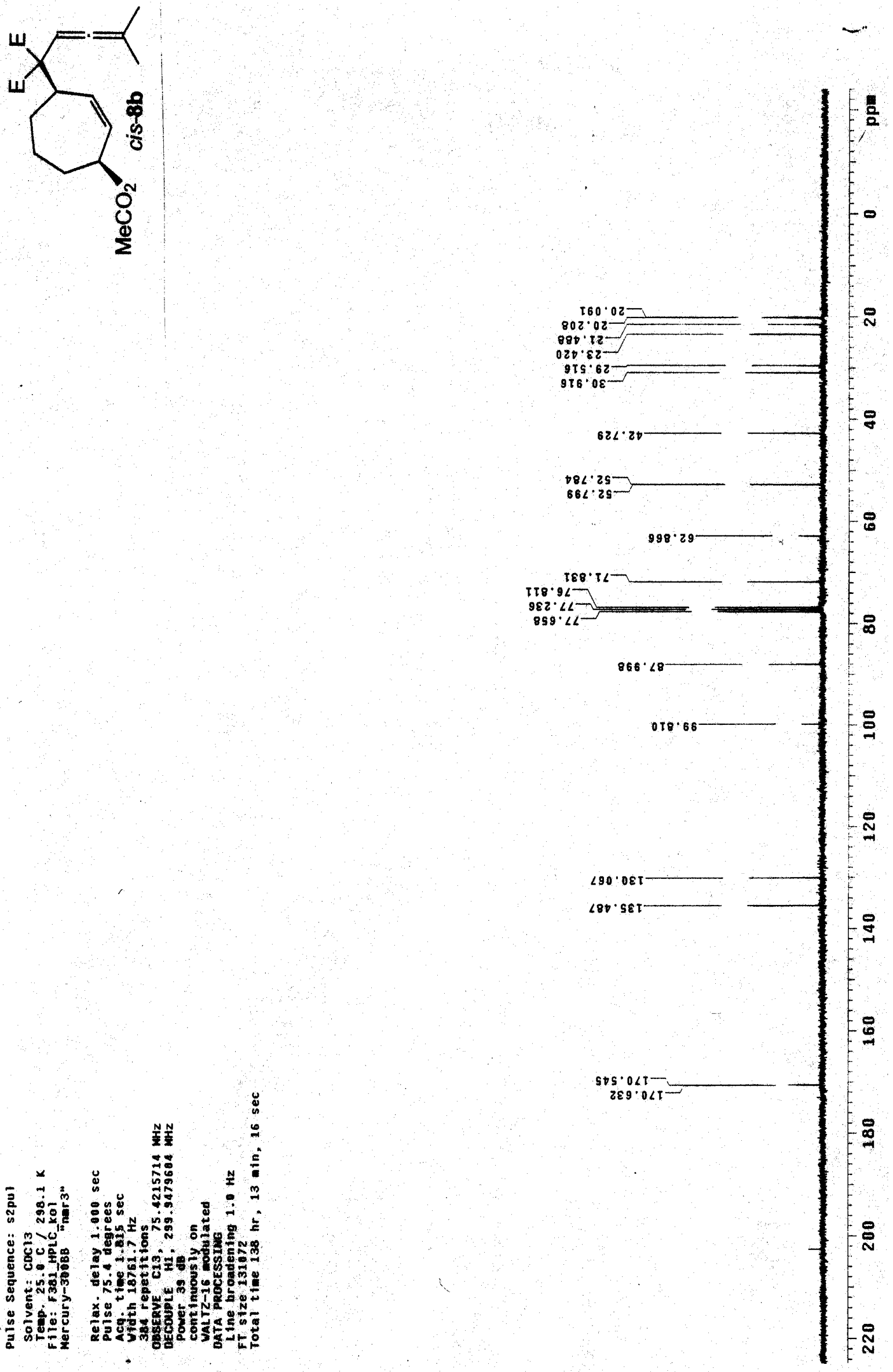

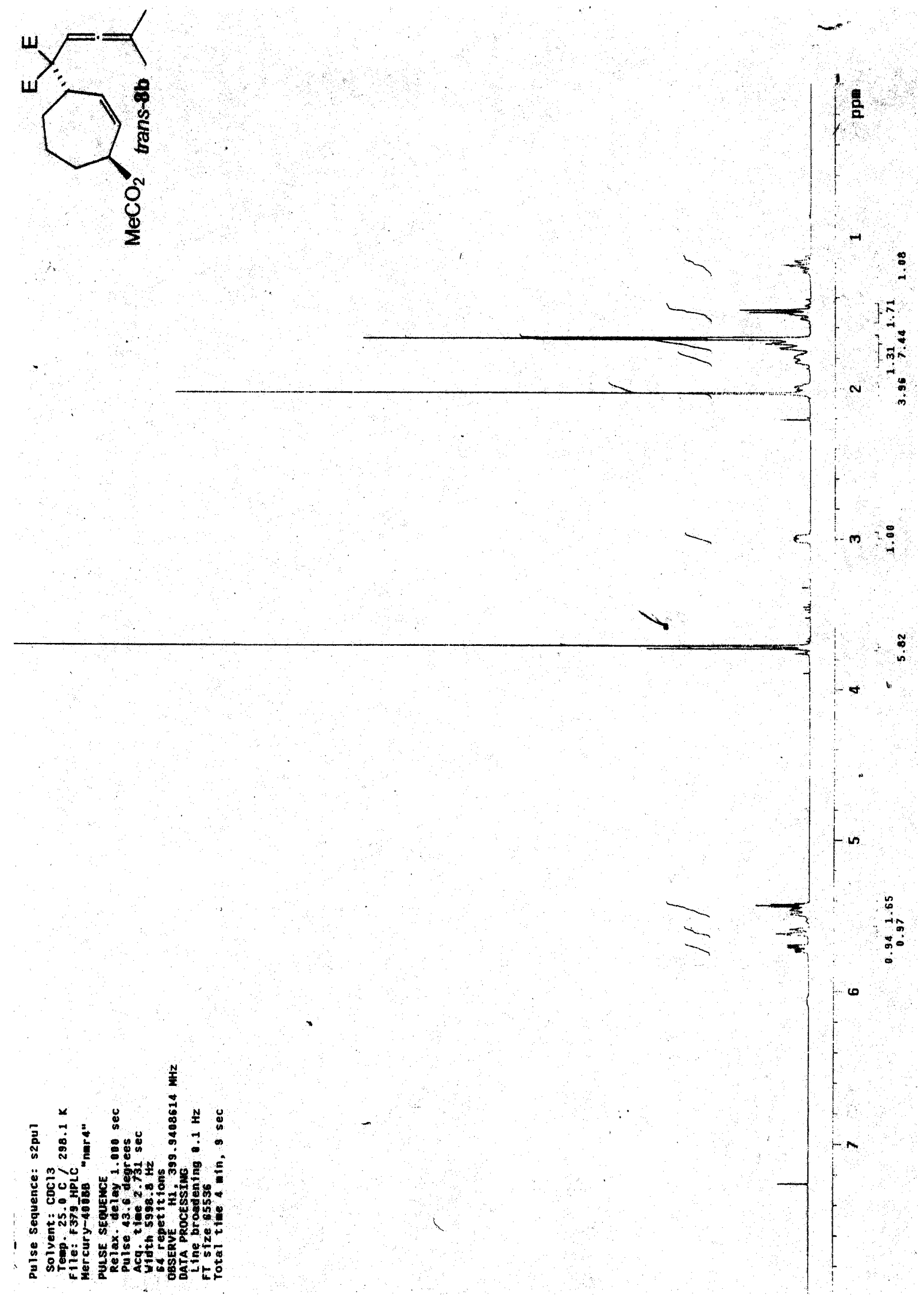

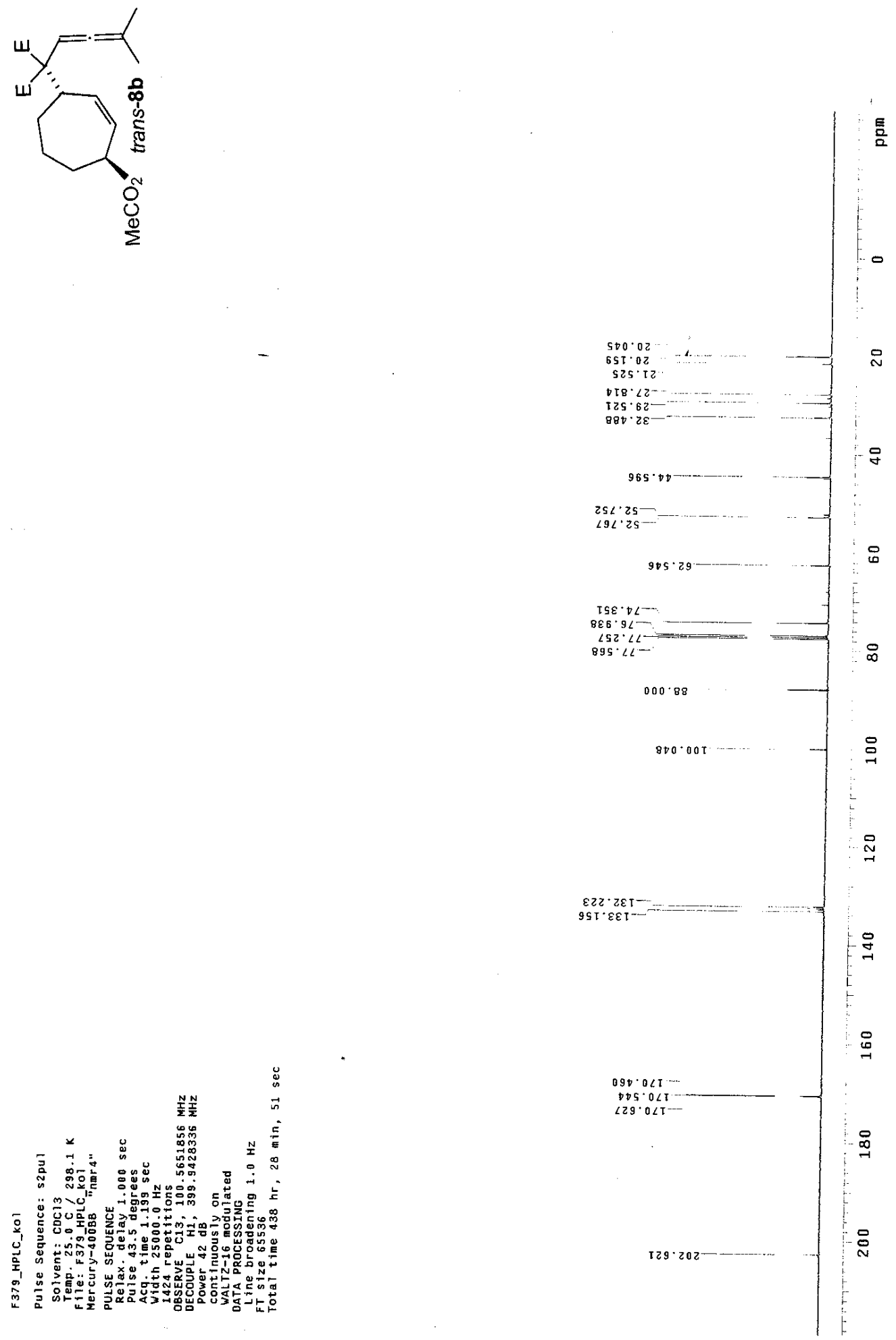

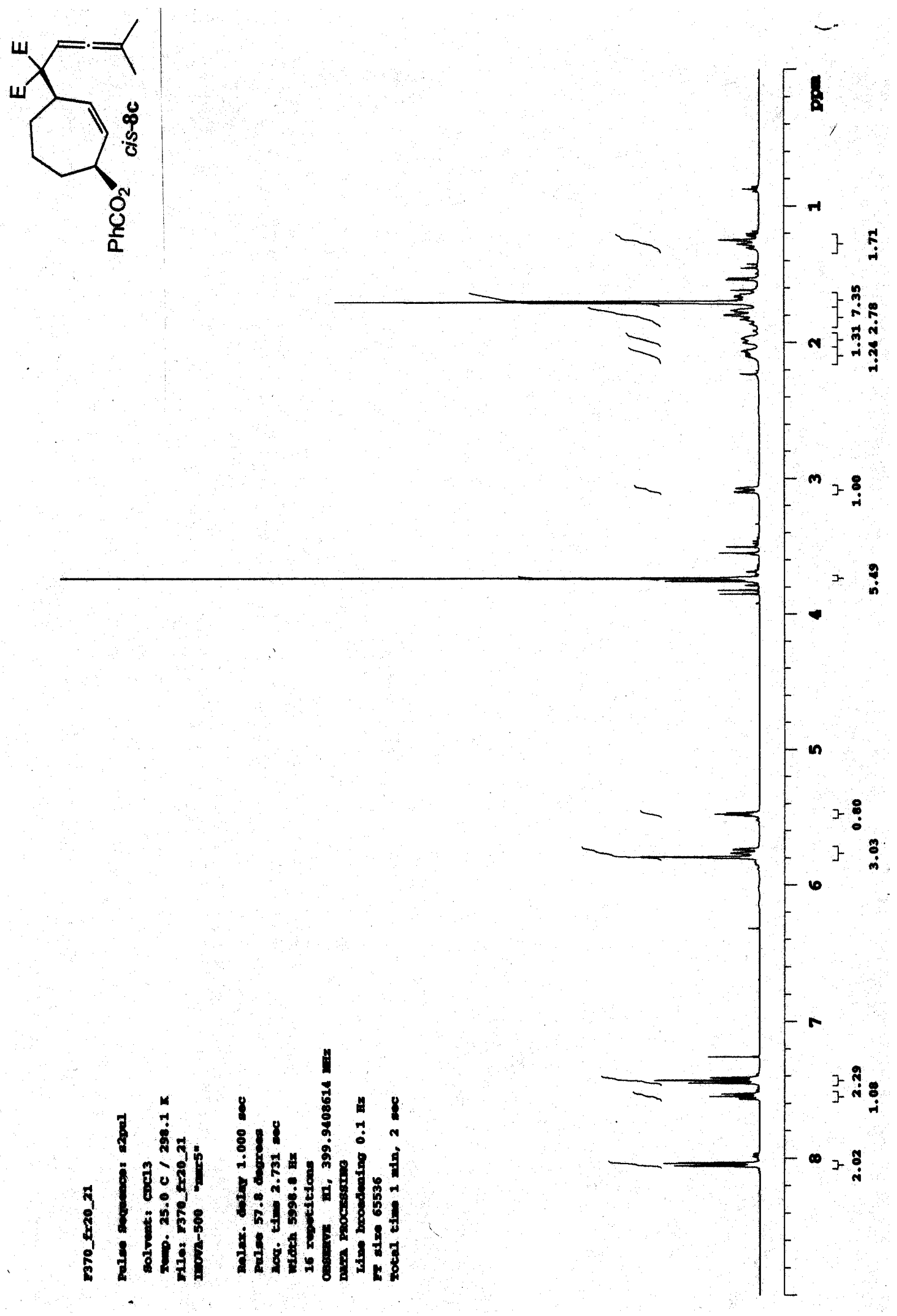

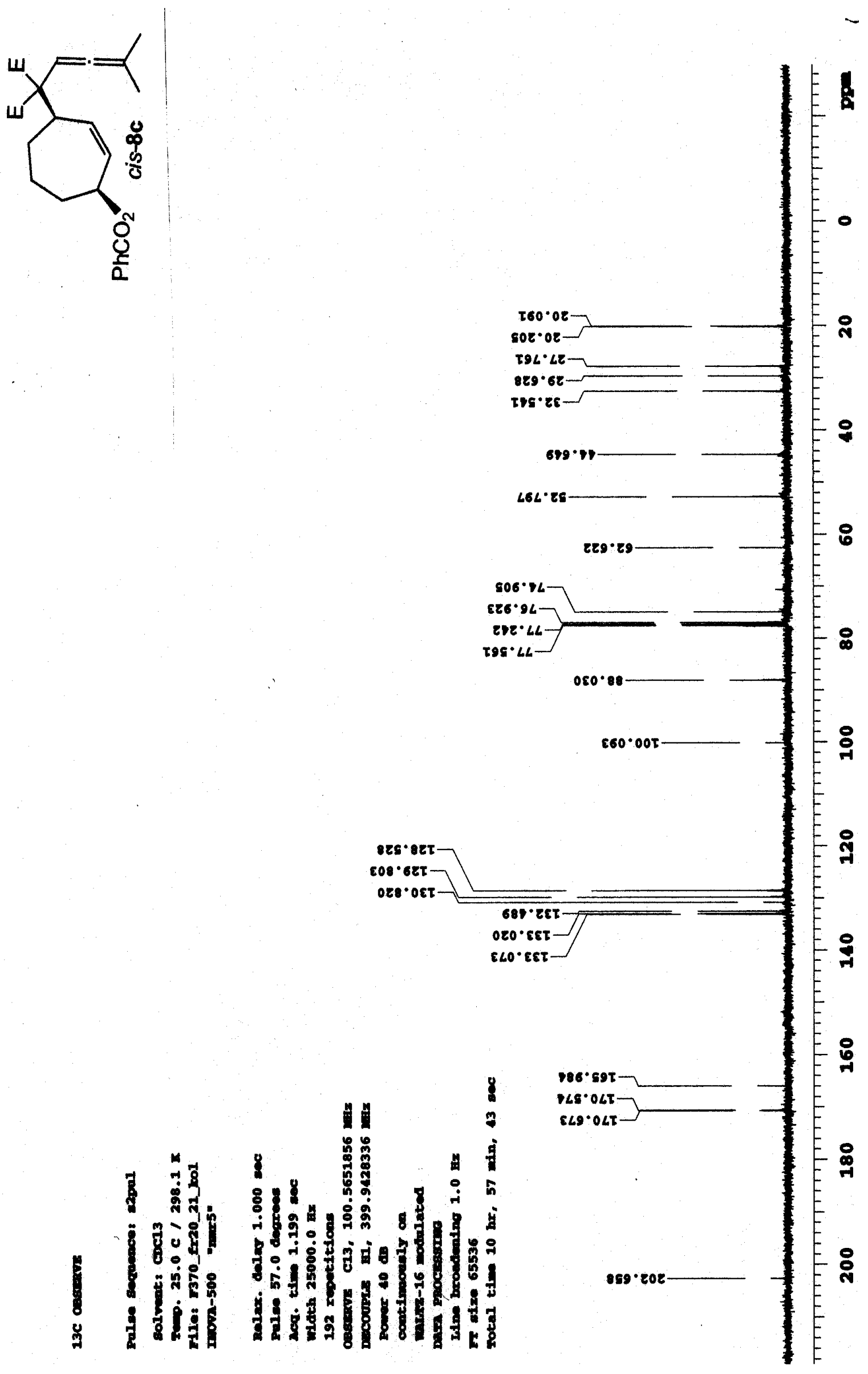
S56
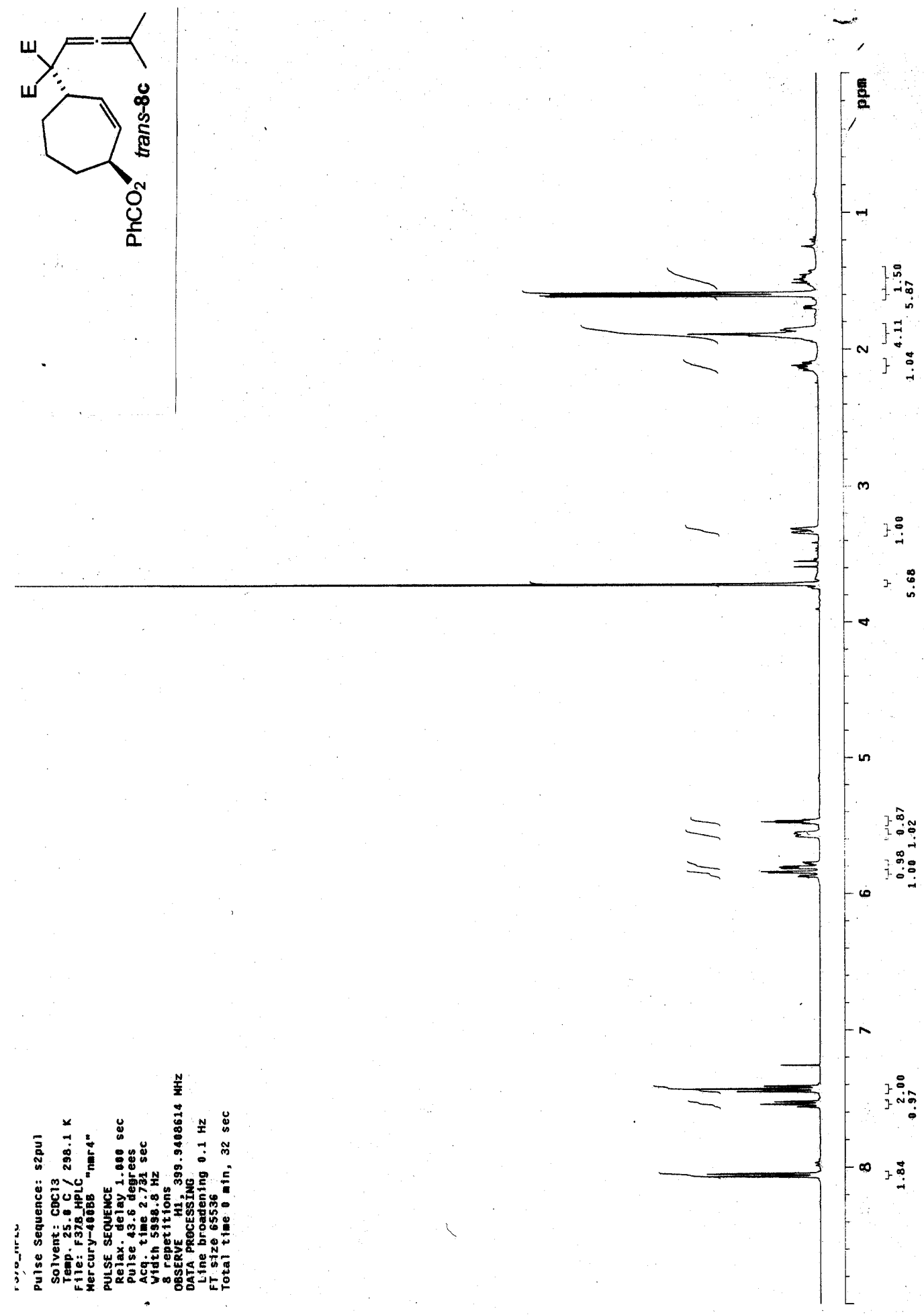

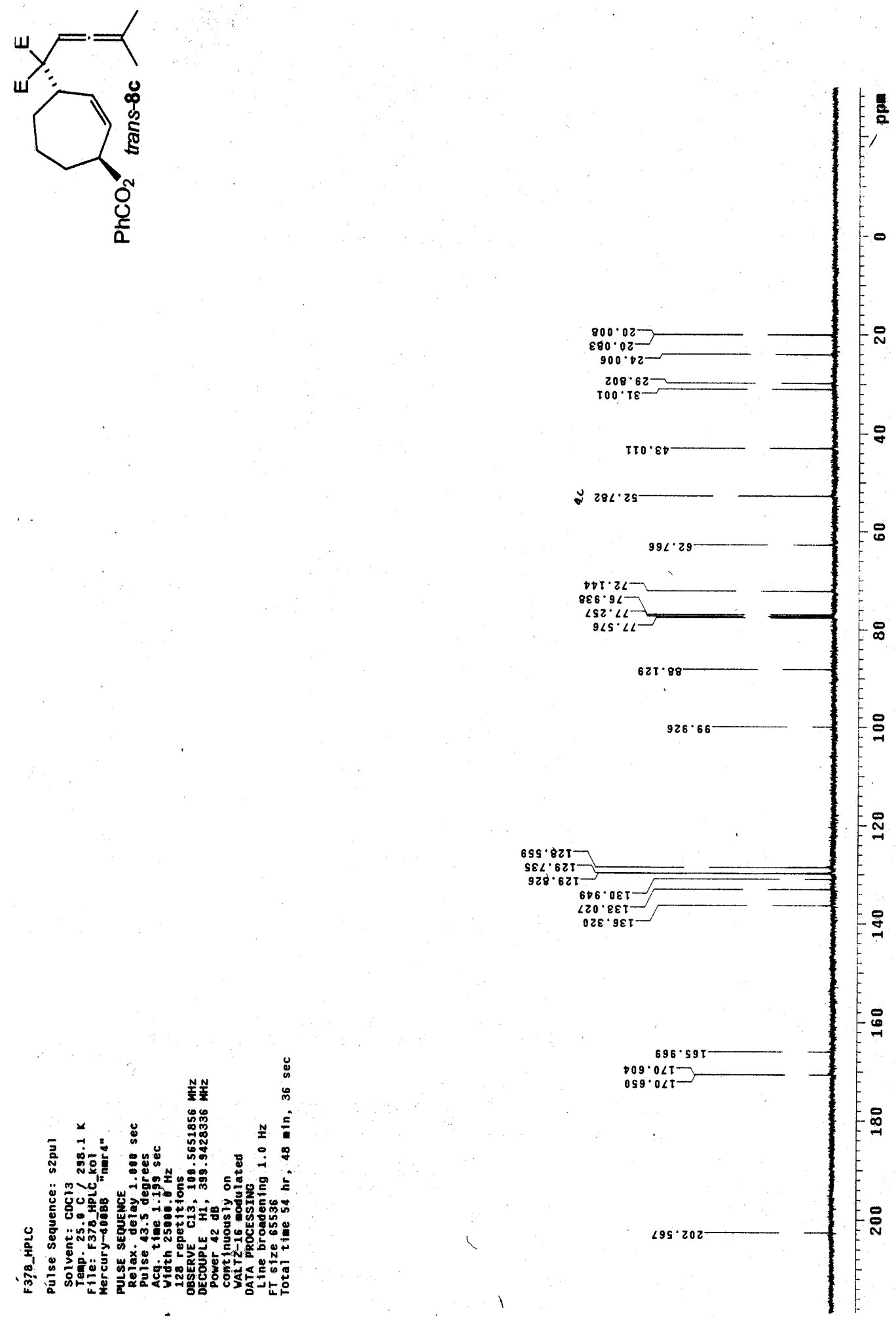


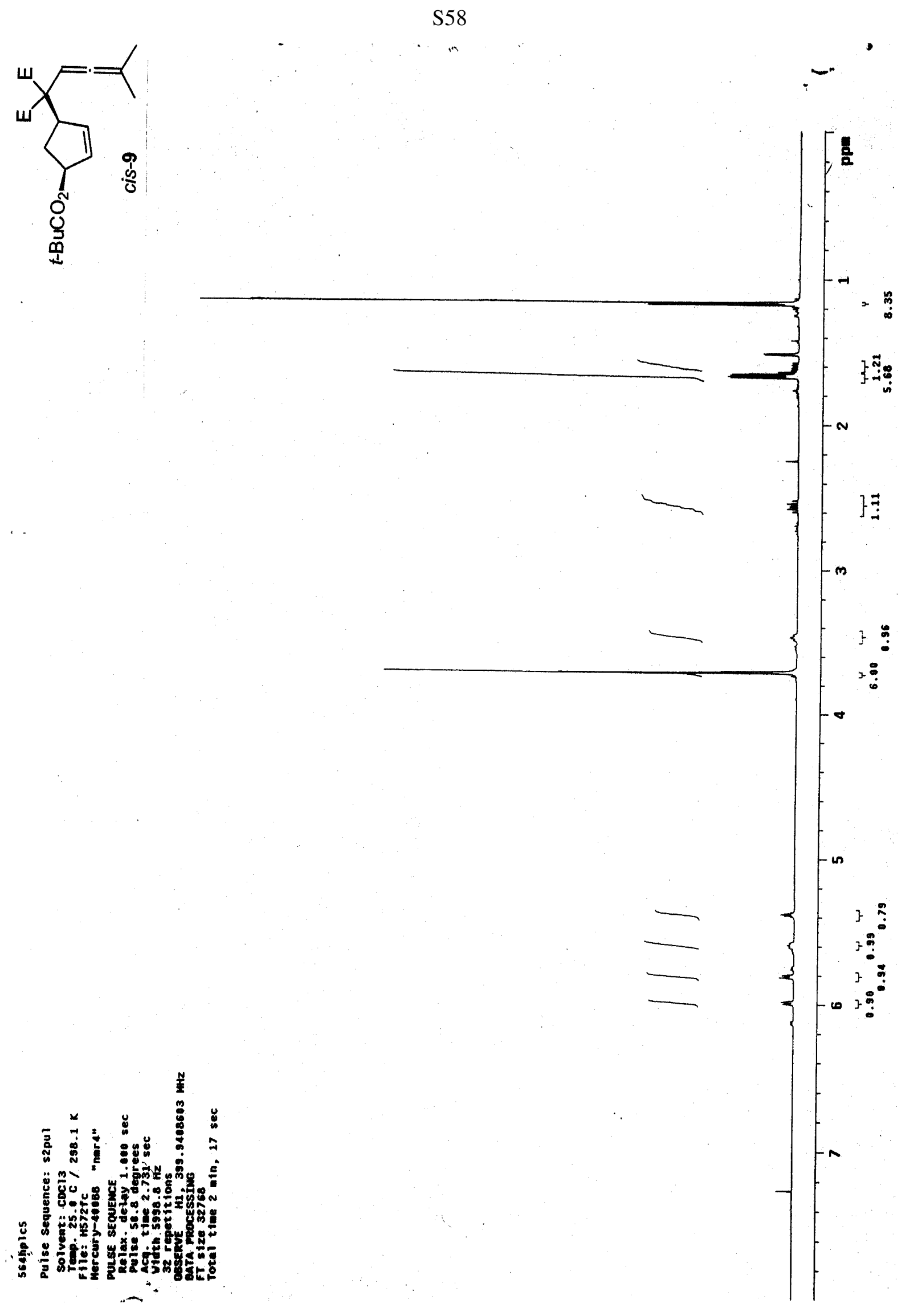



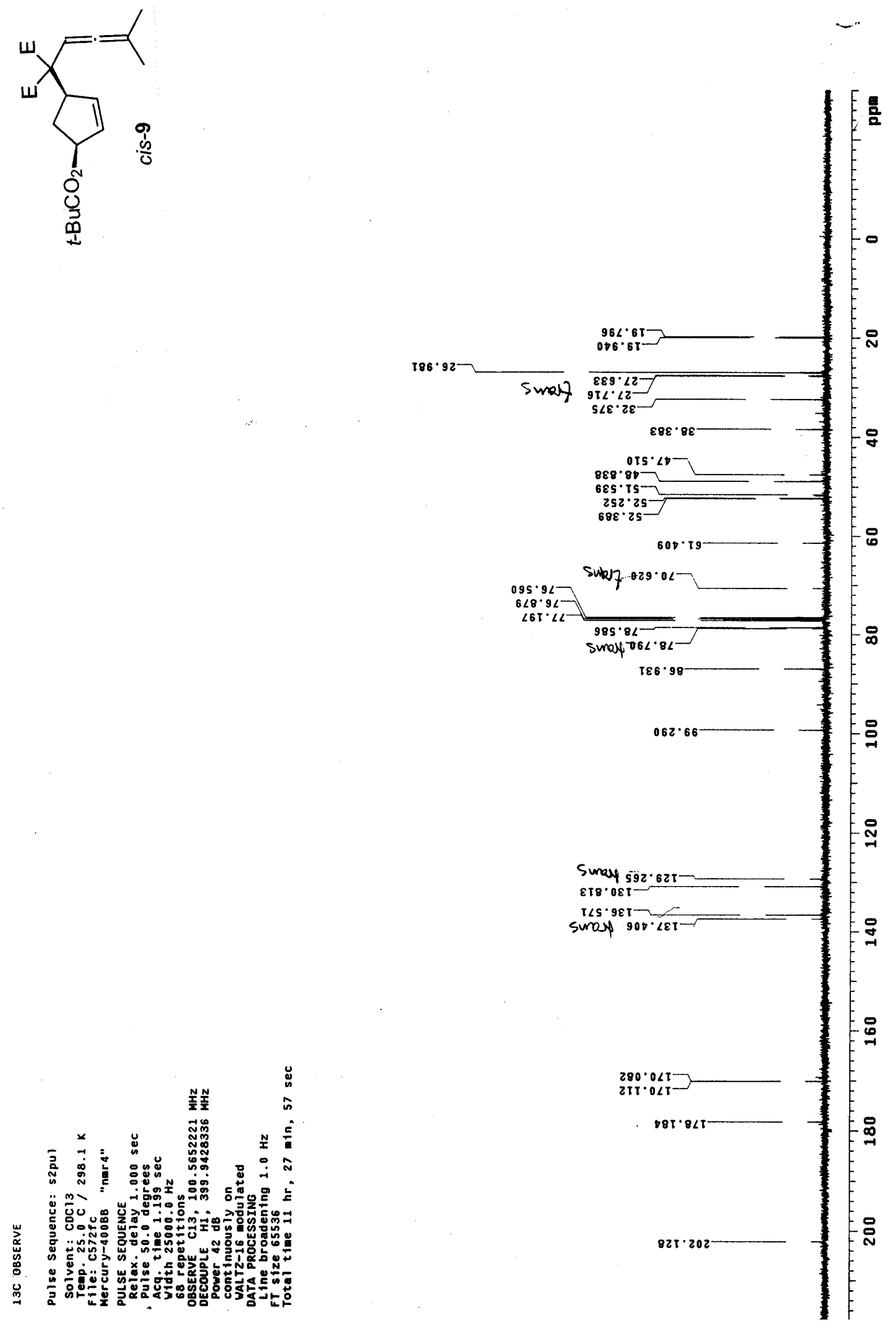


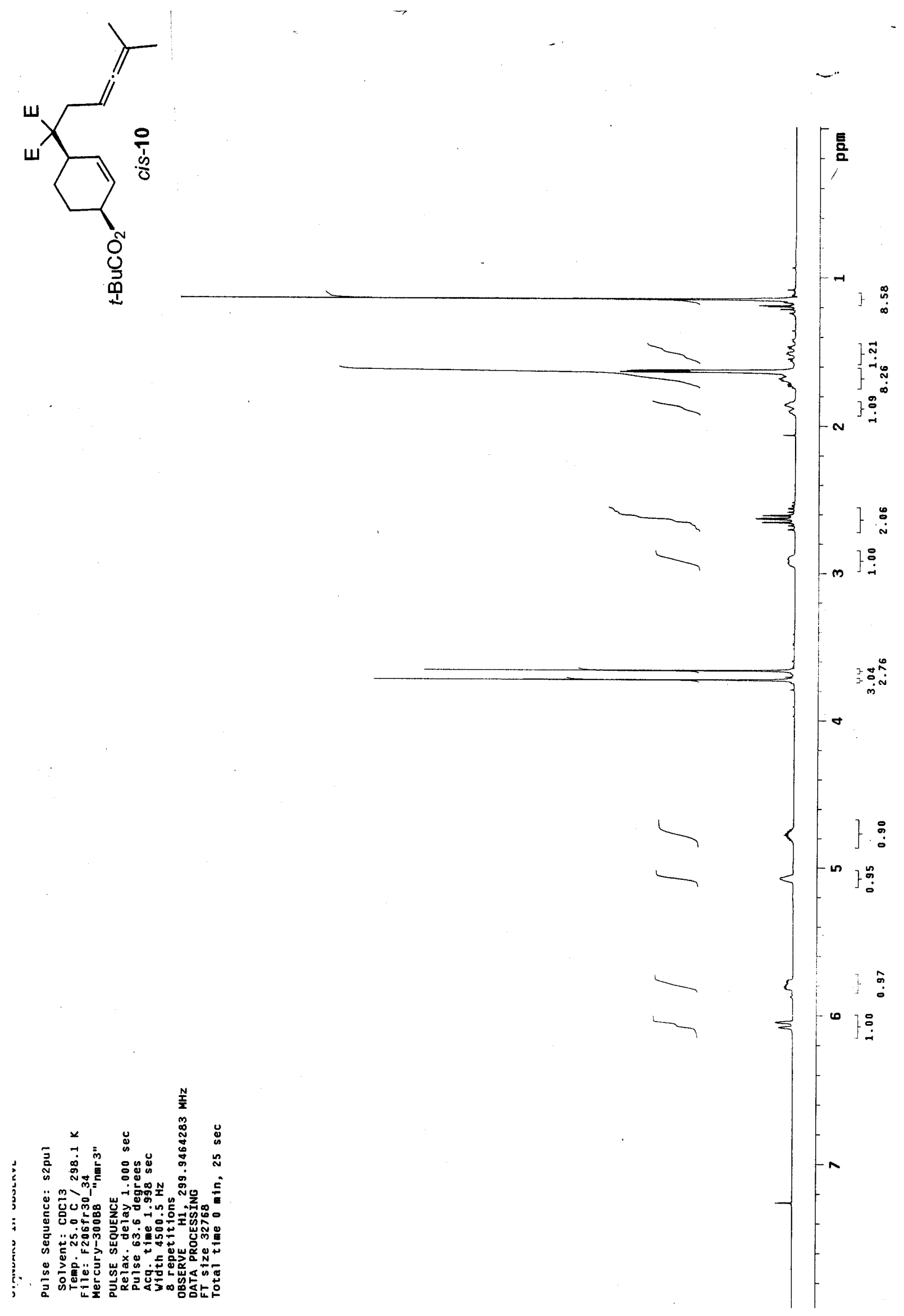



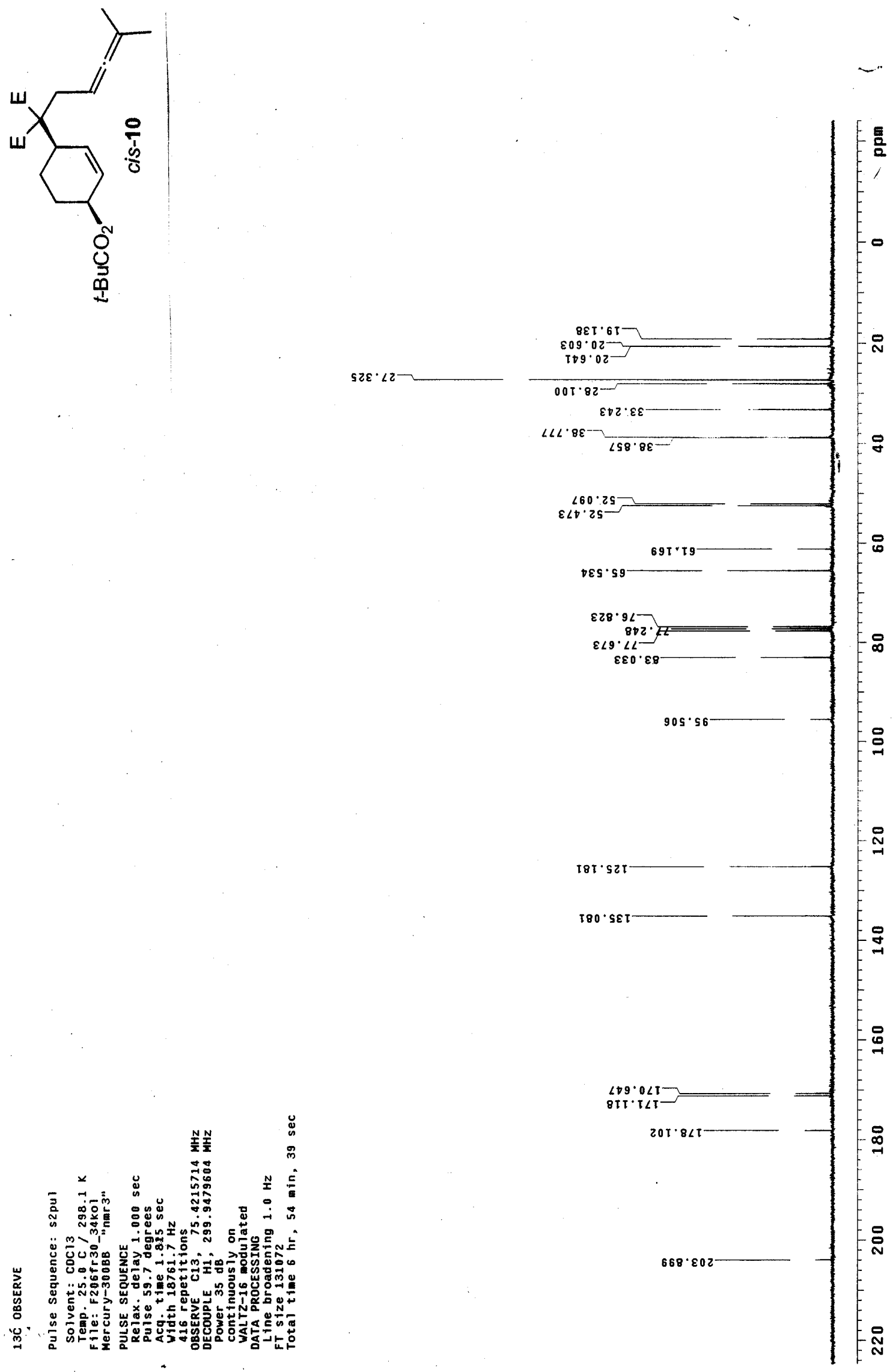


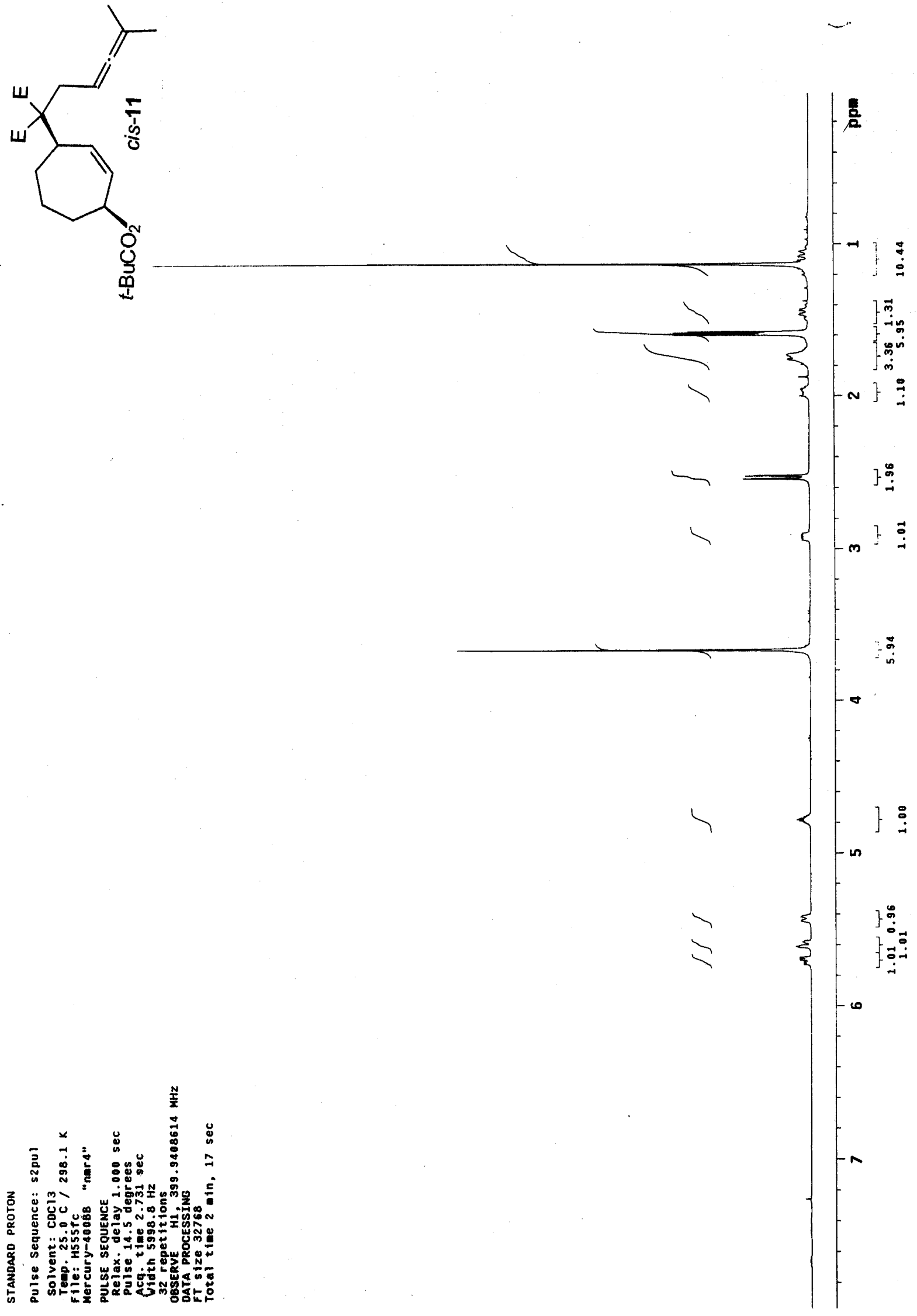



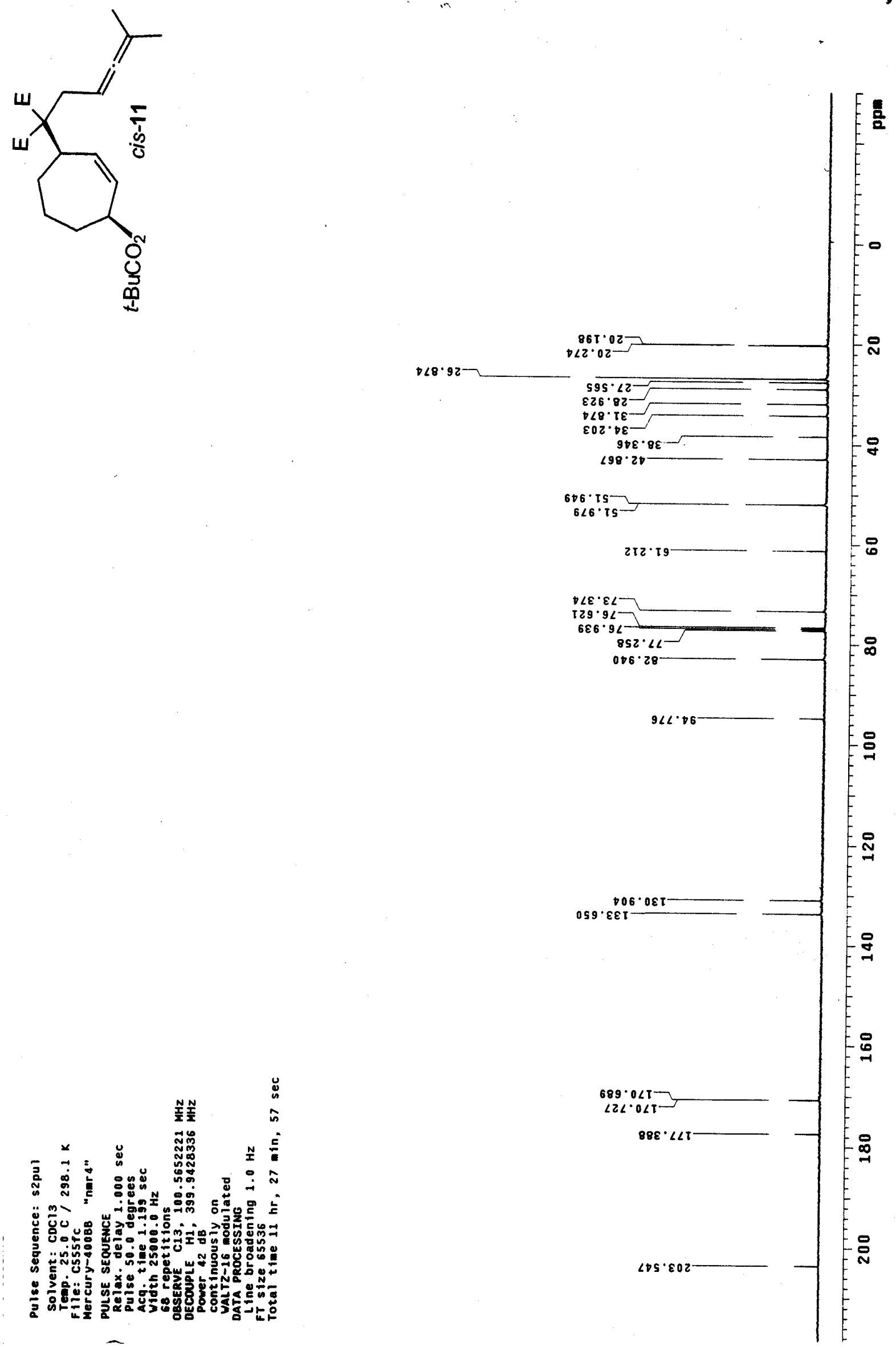
S64

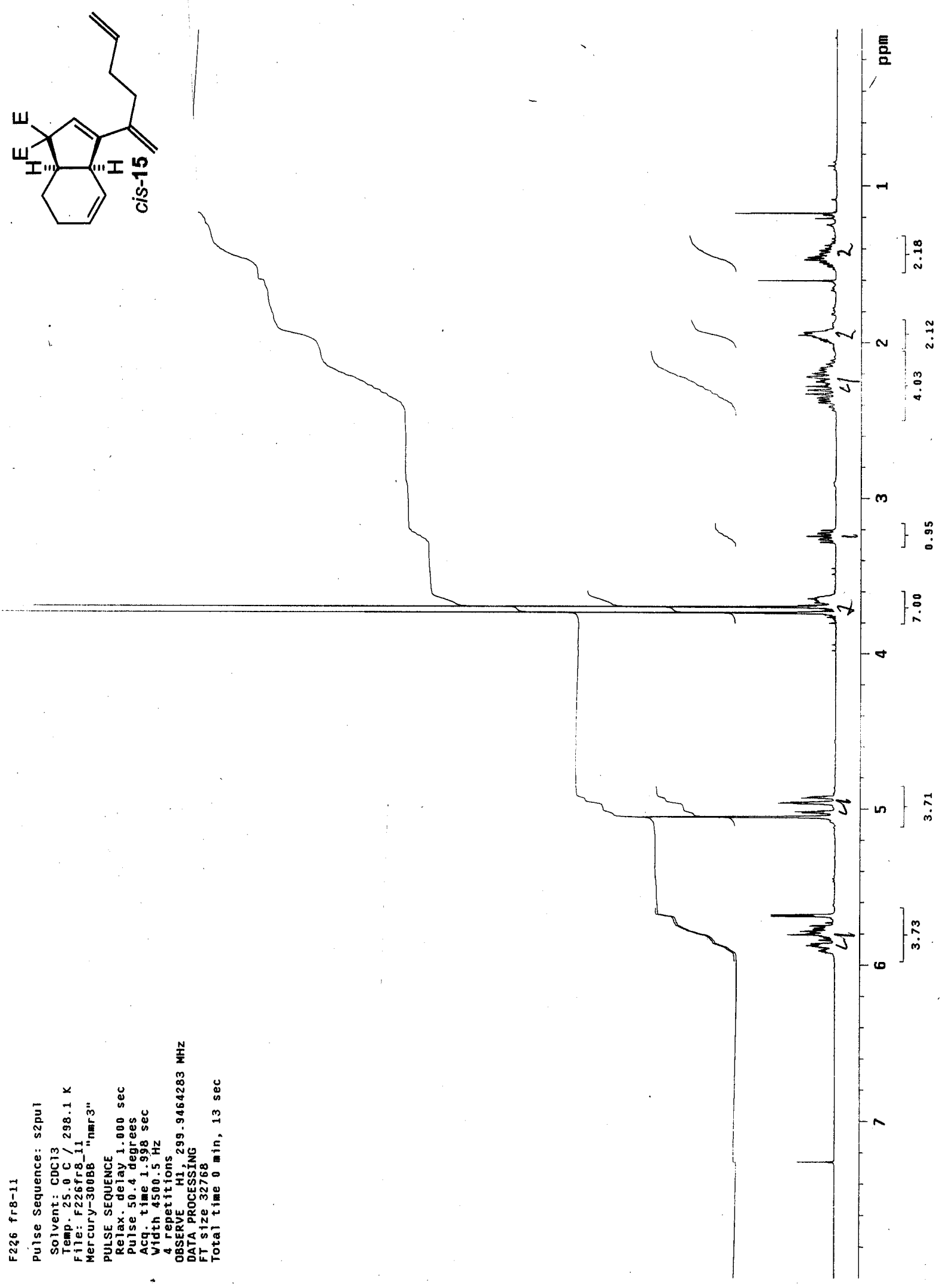



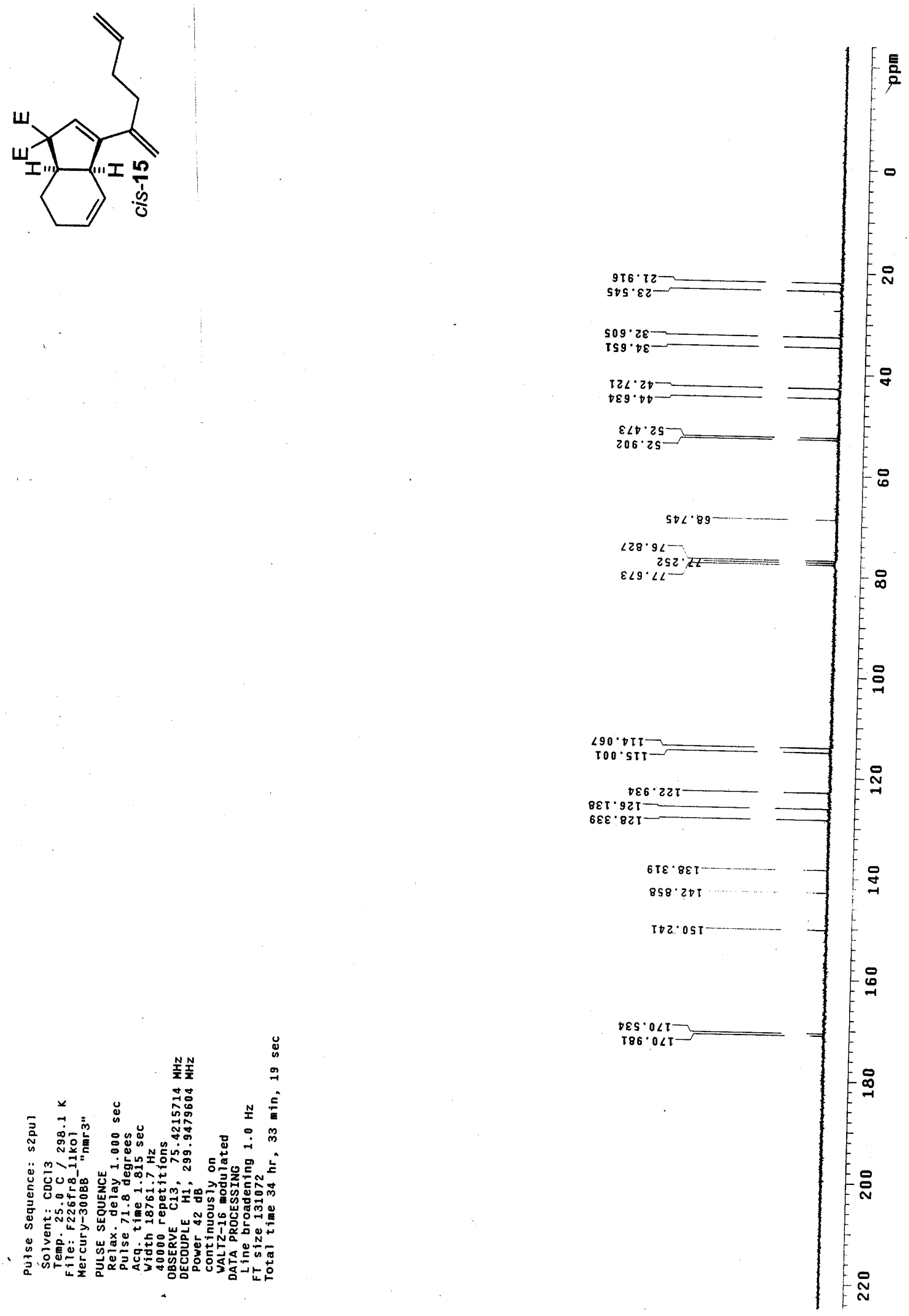

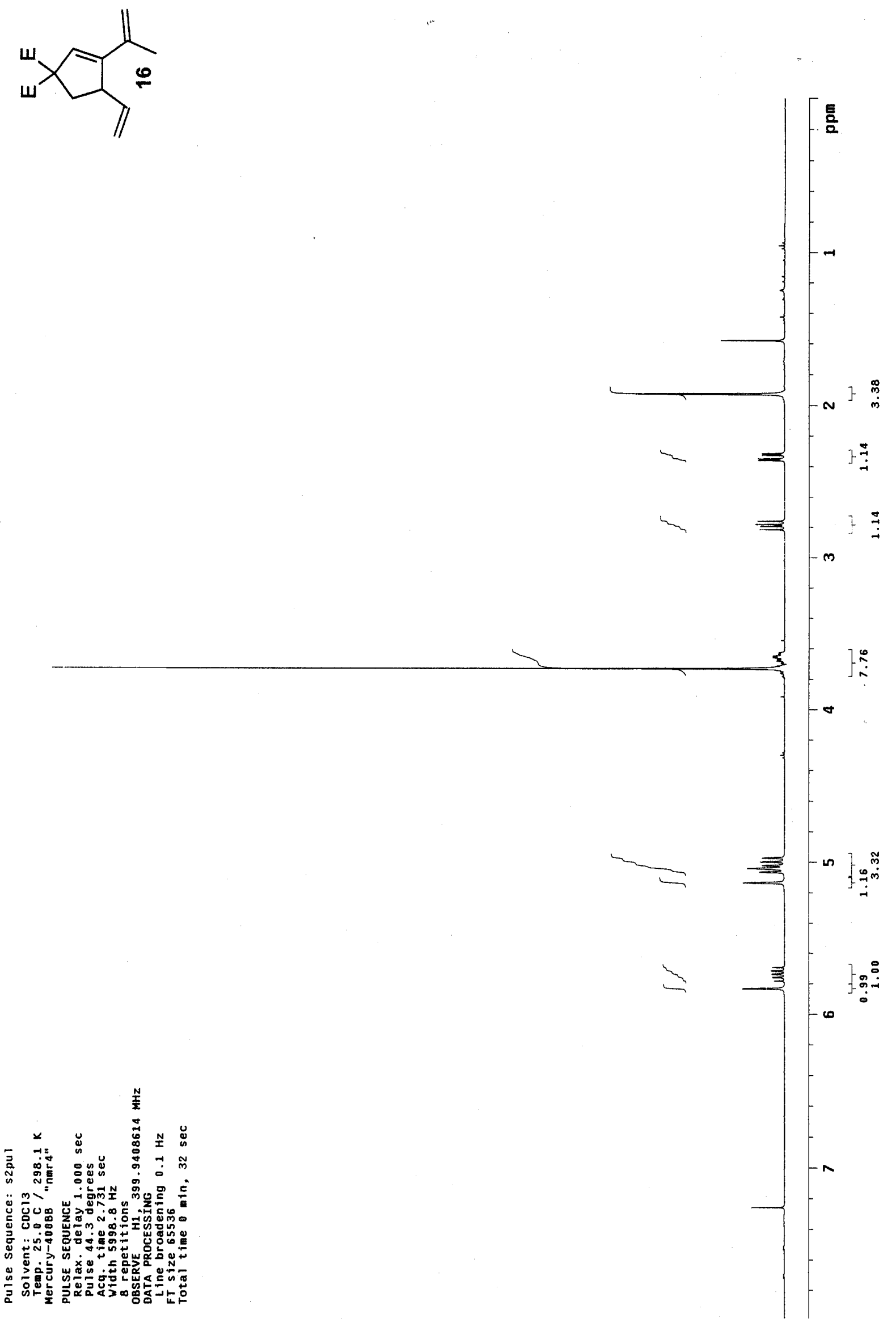

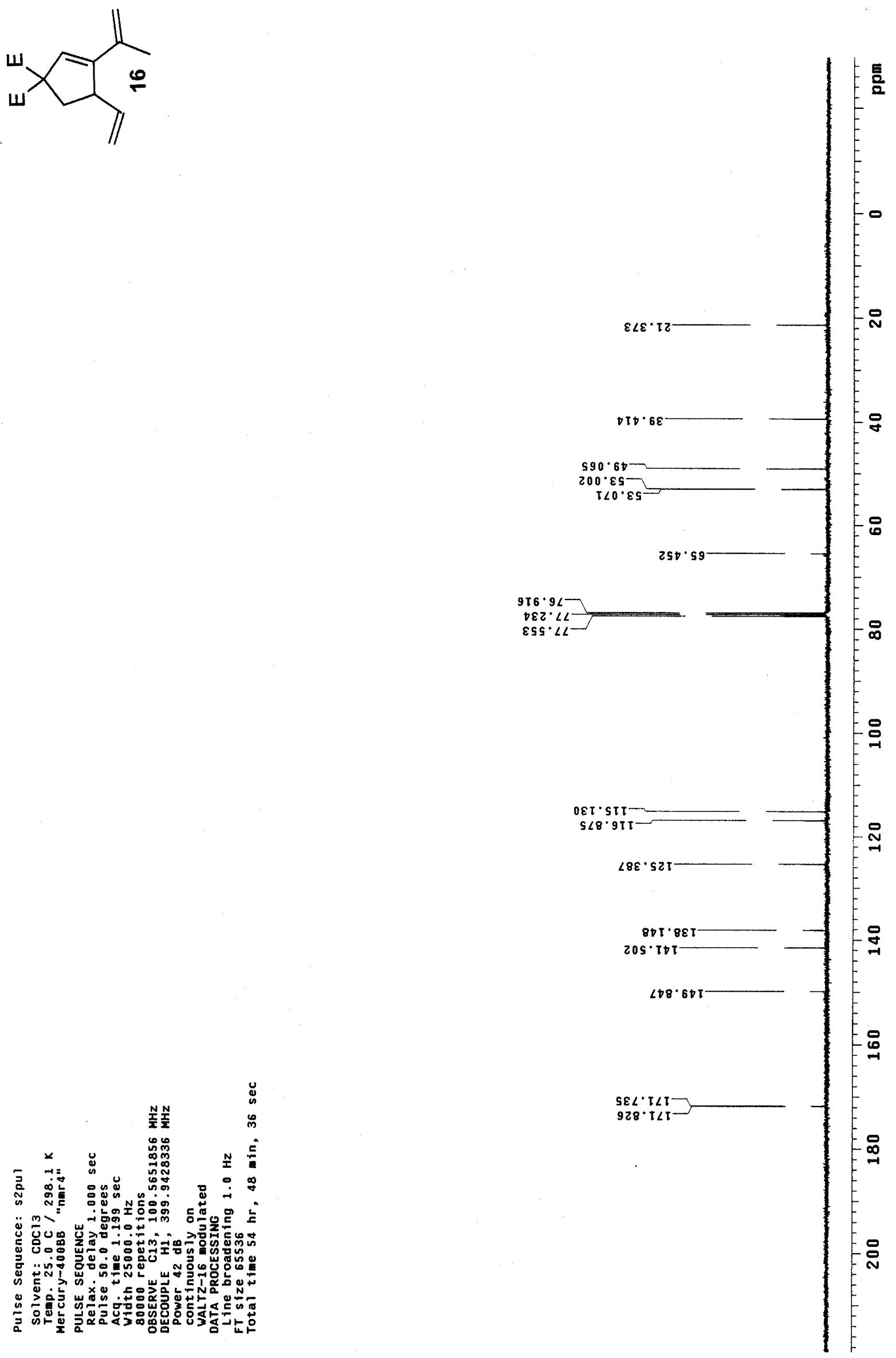

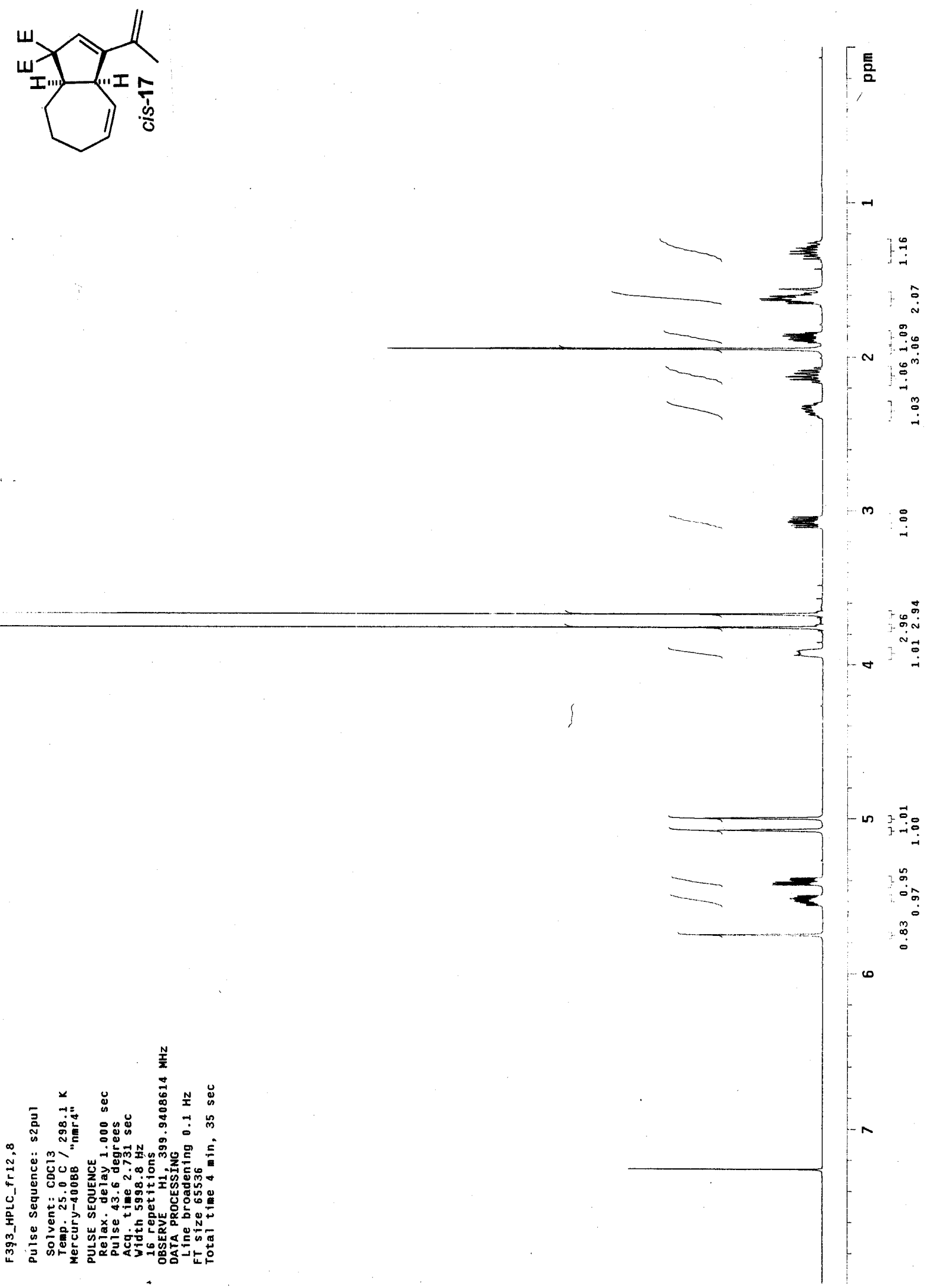

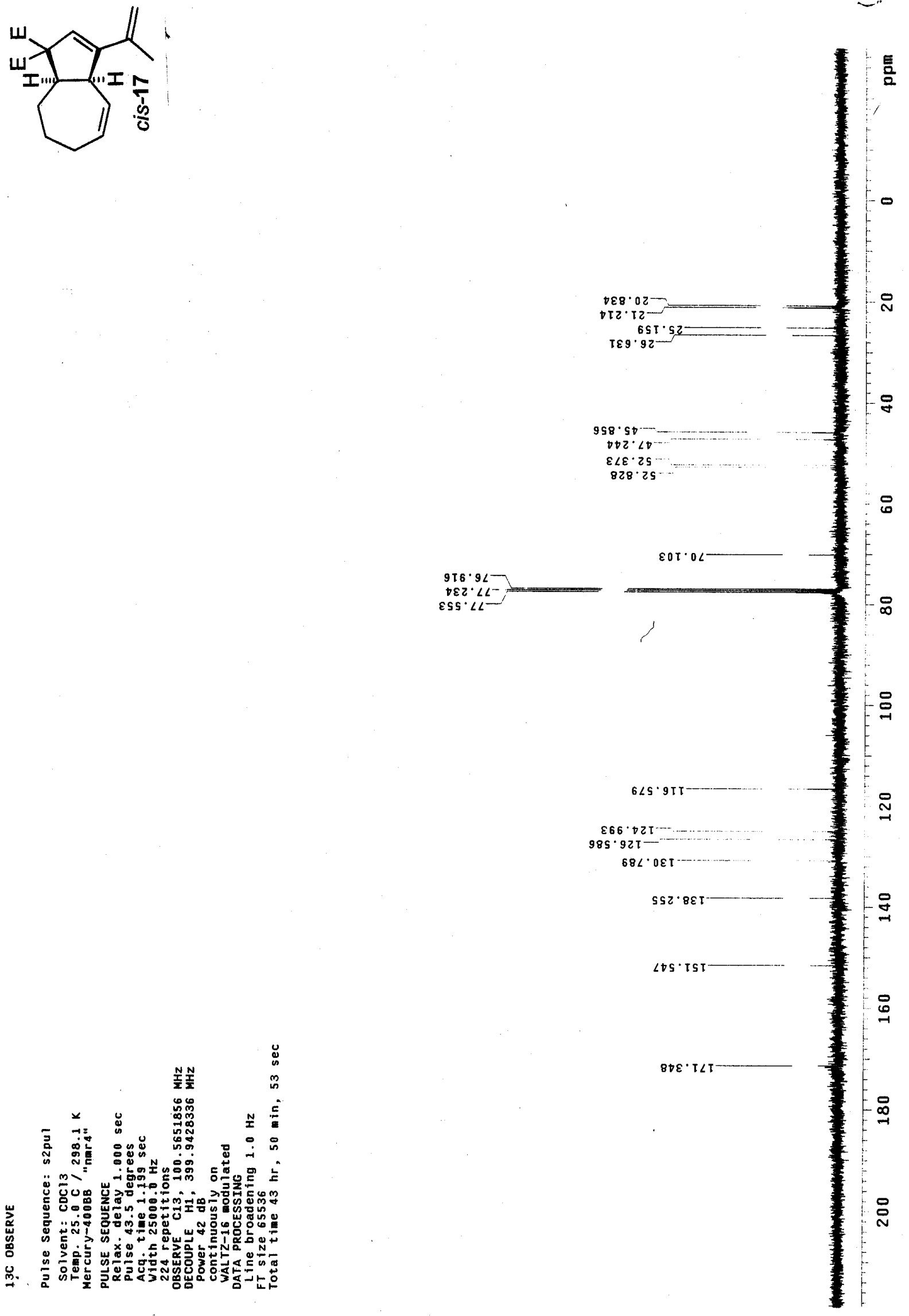
S70

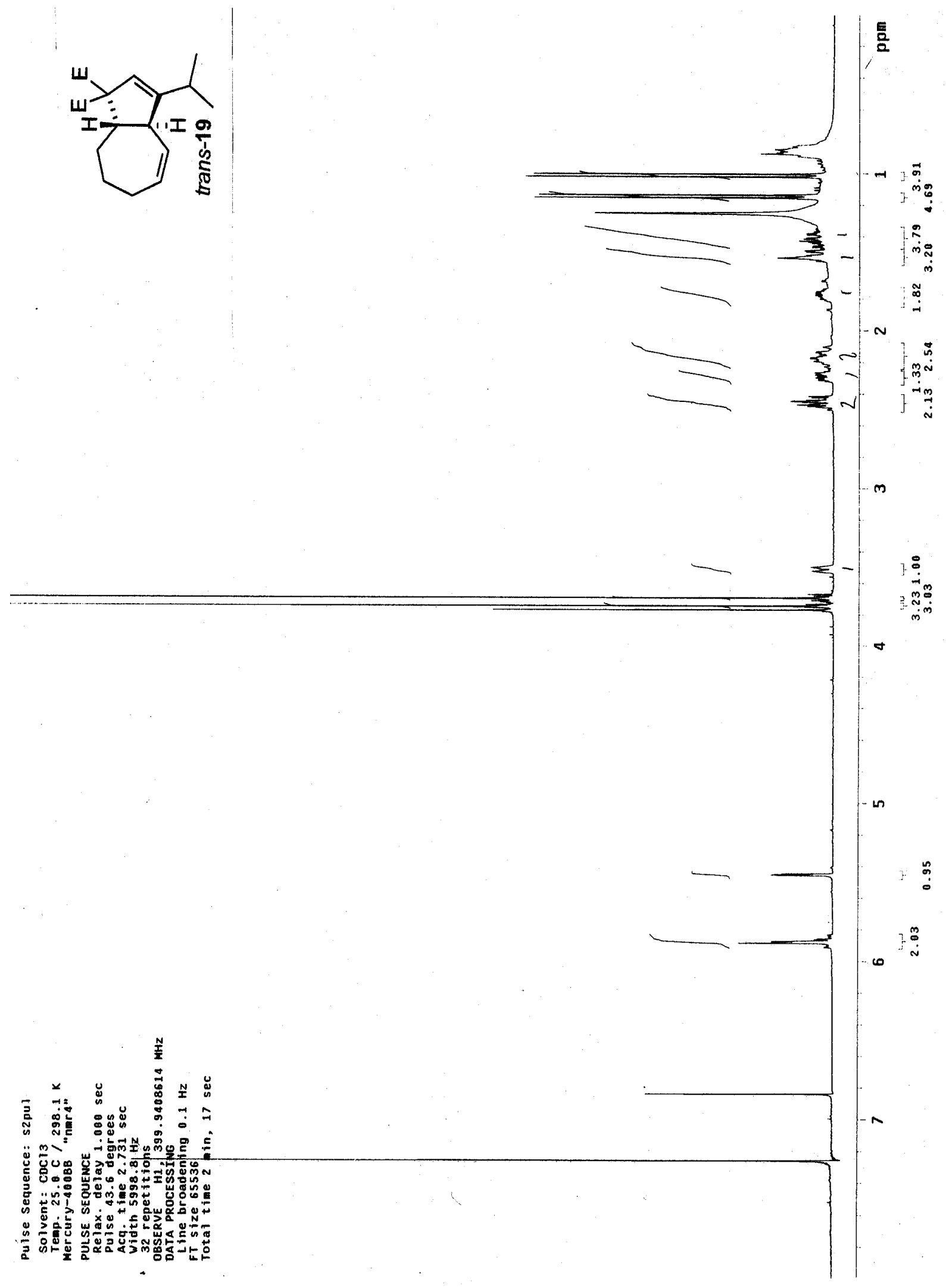



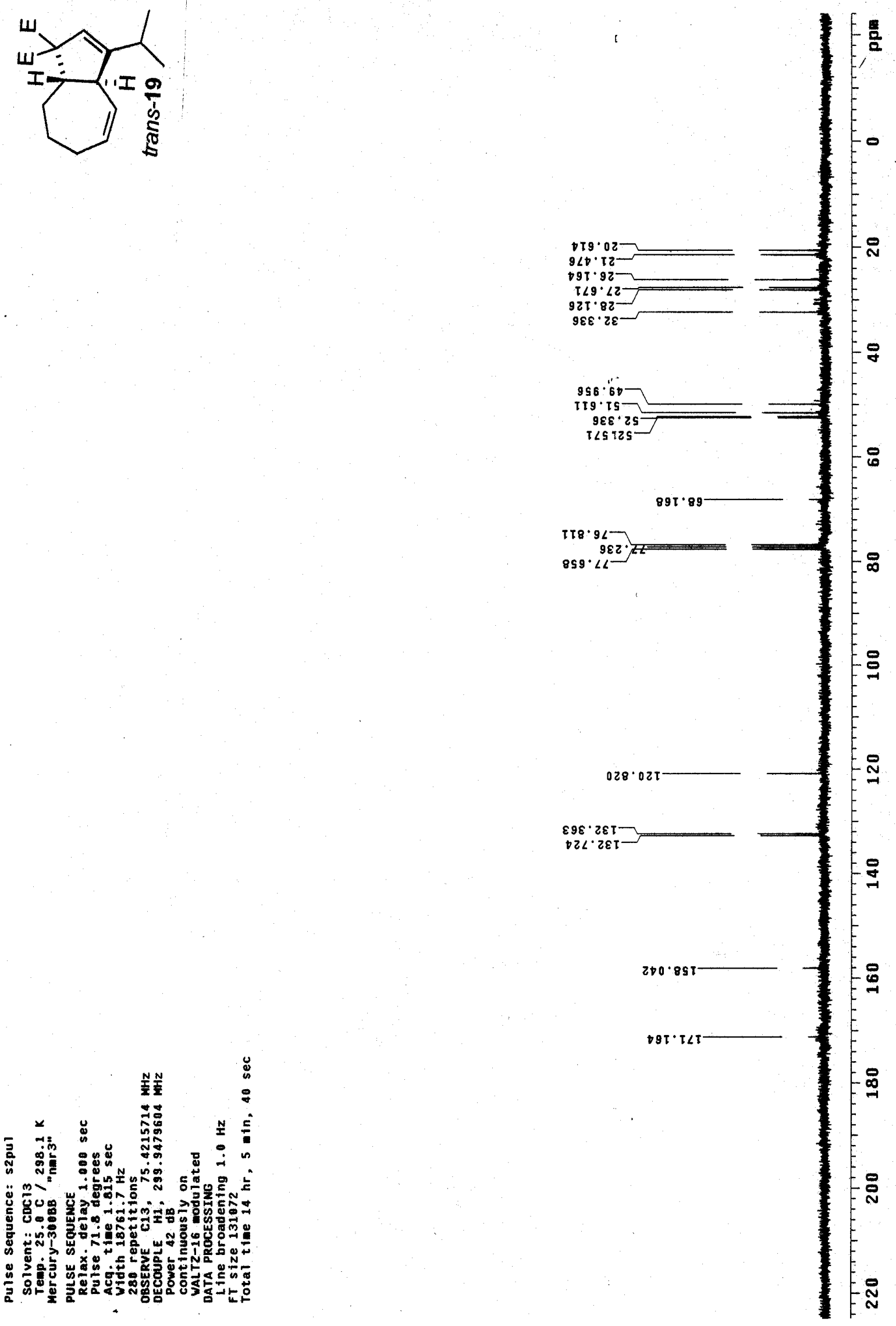


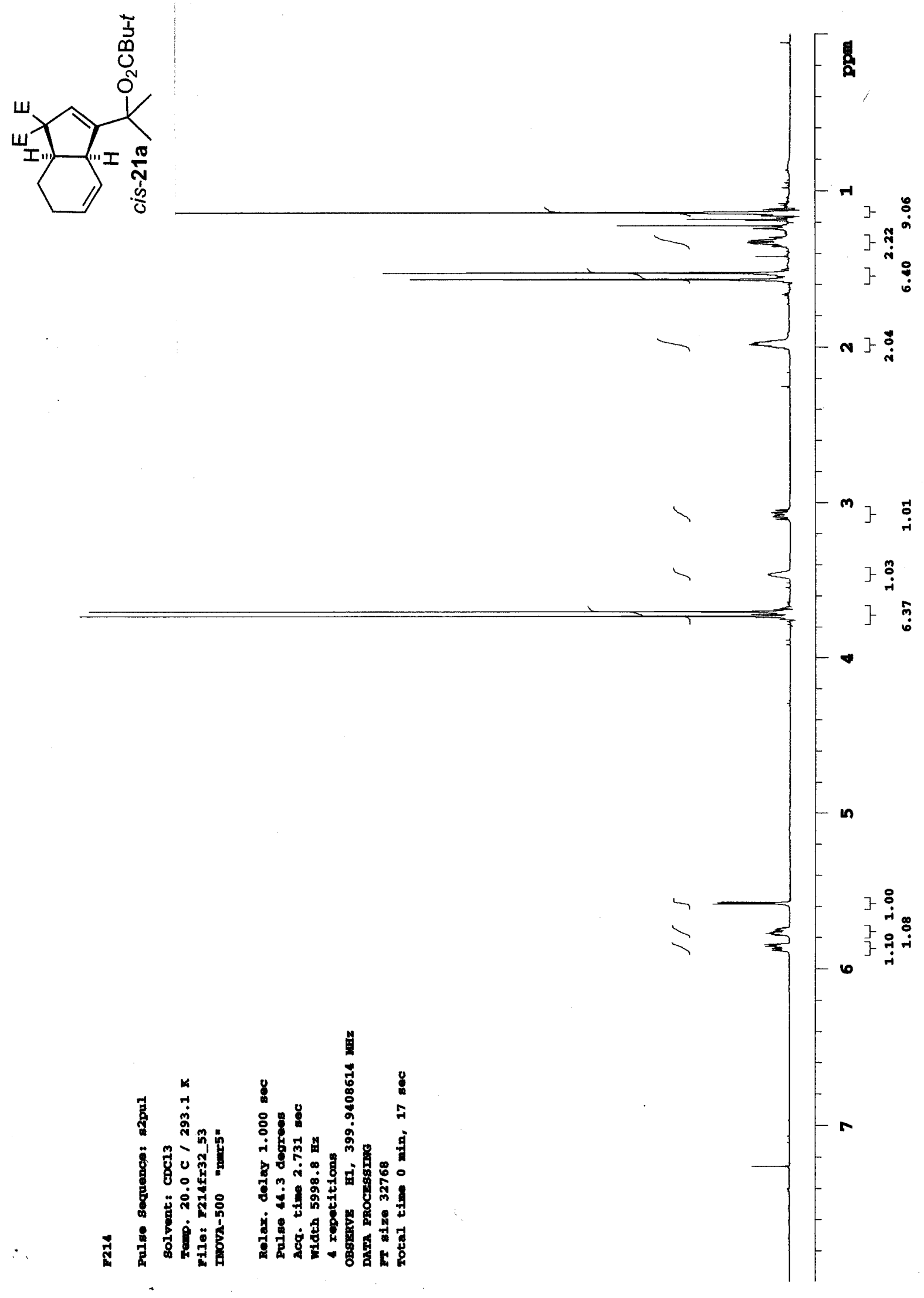



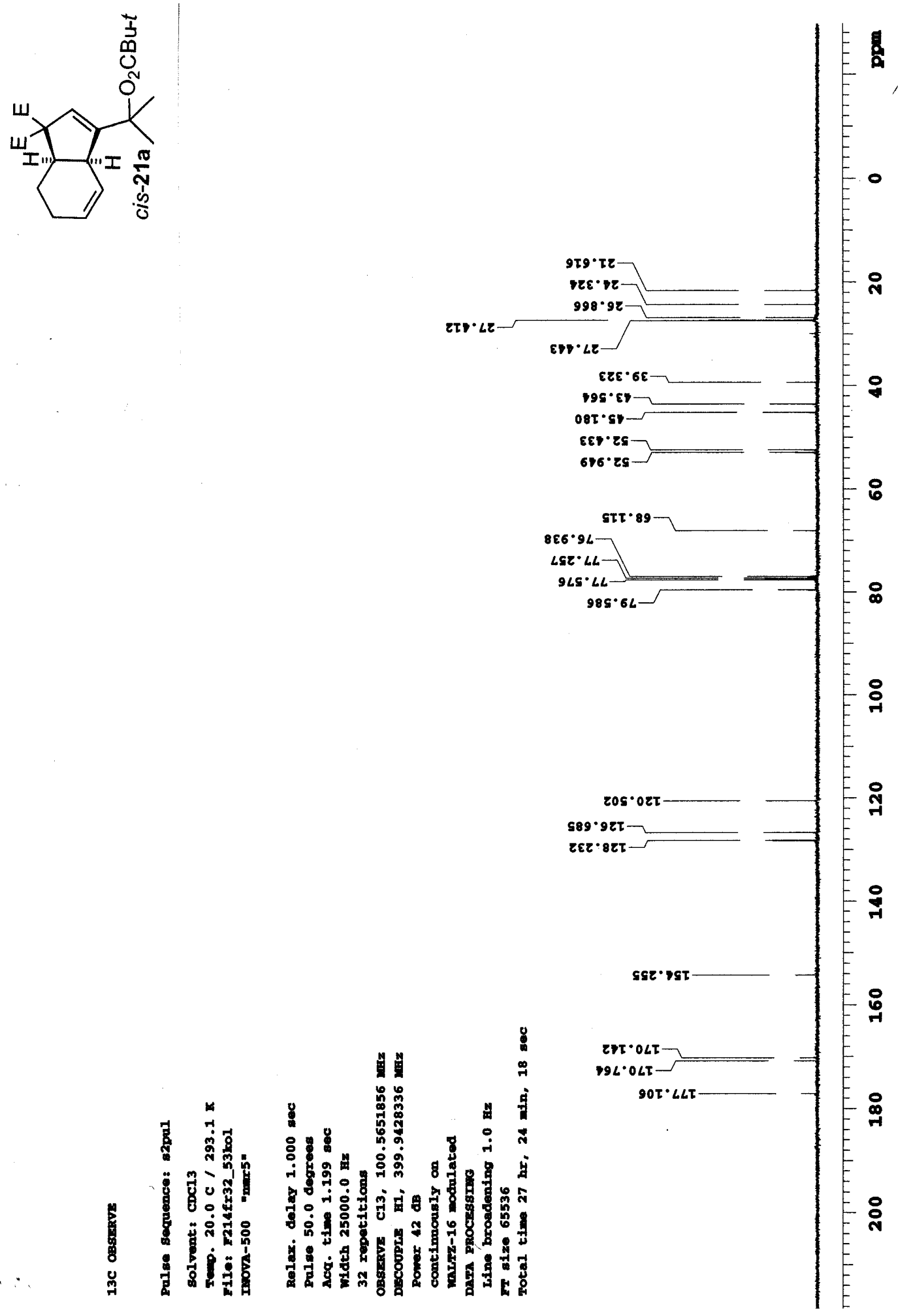

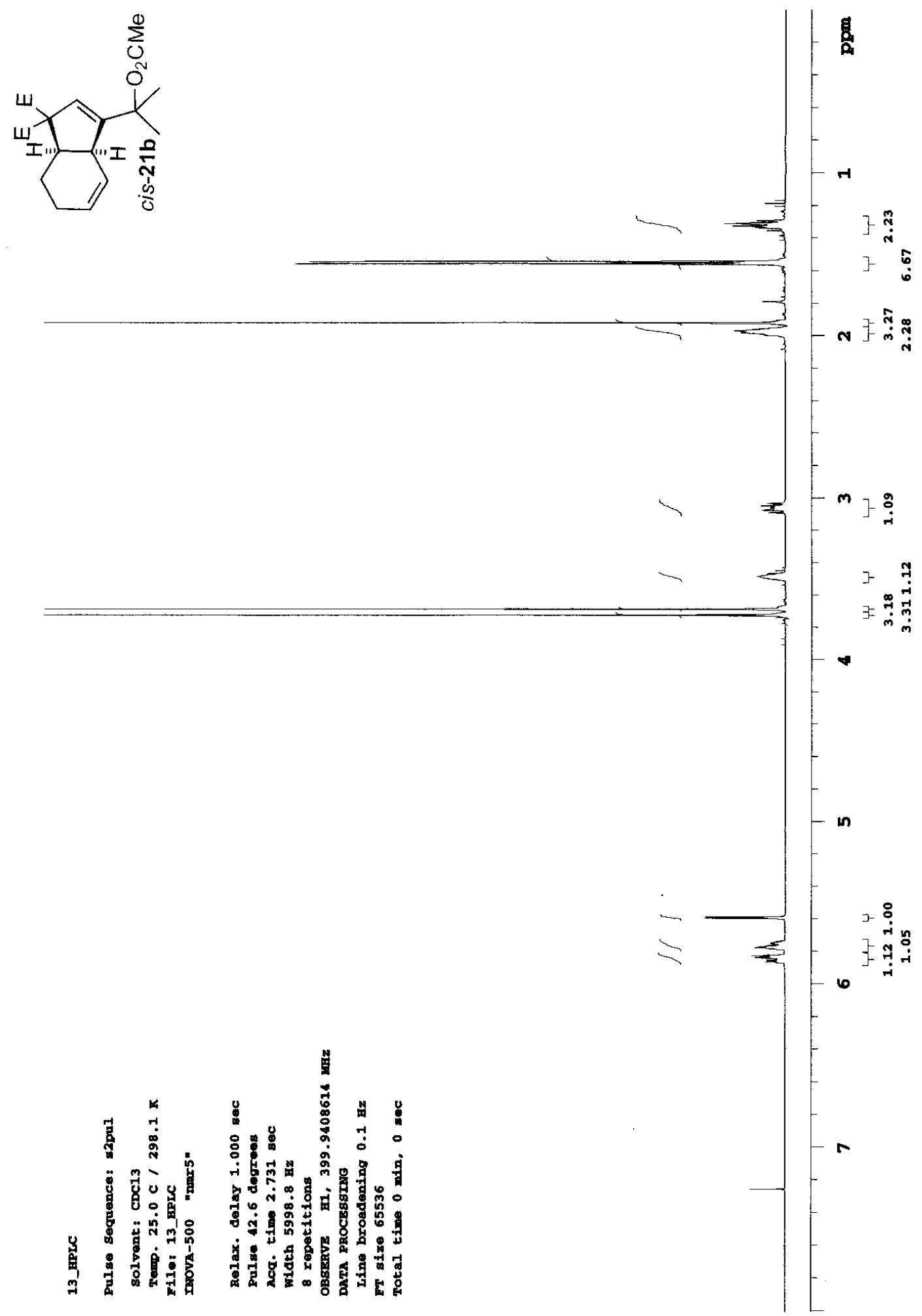

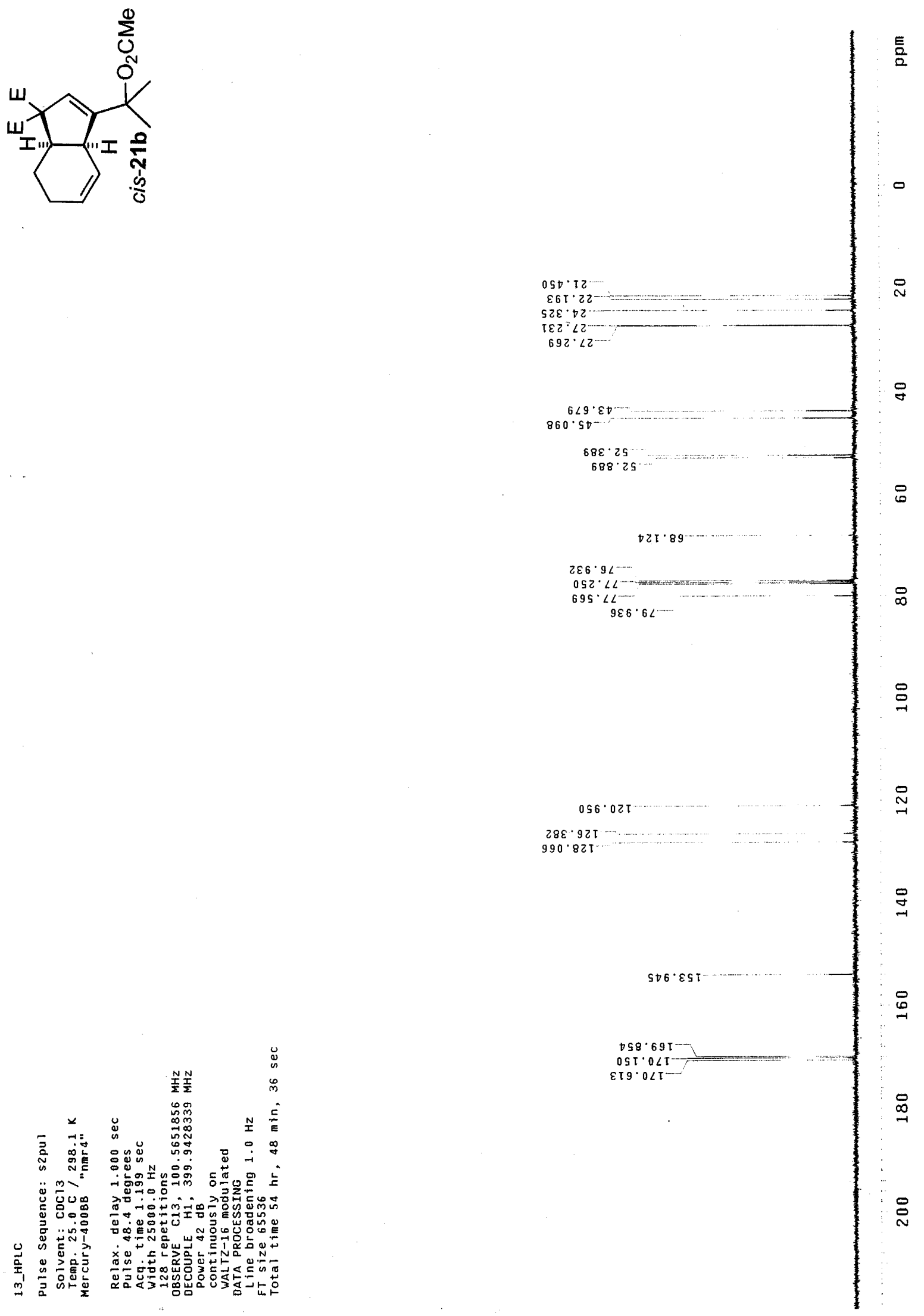

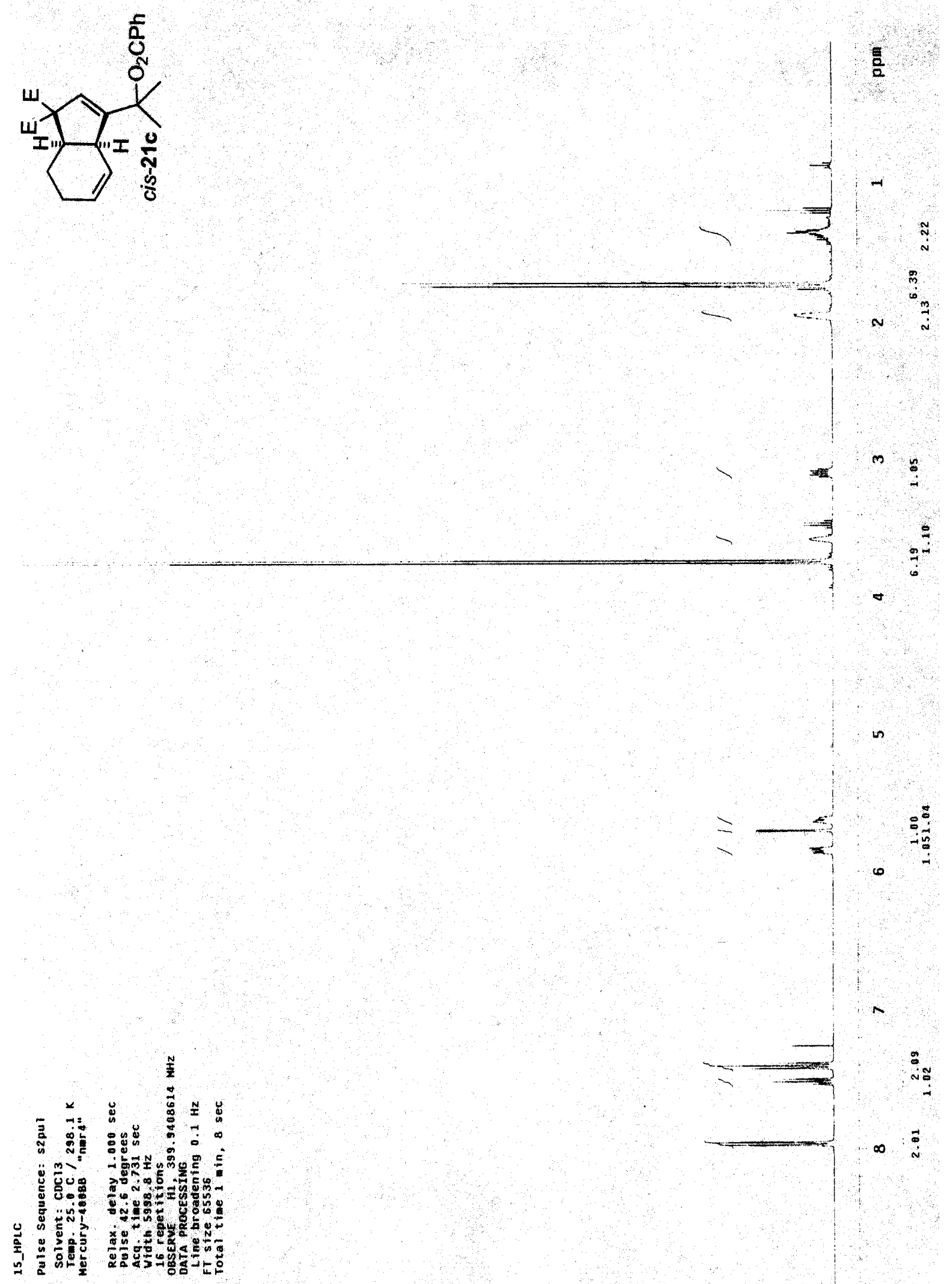

$\infty \stackrel{\overrightarrow{0}}{\sim}$ 

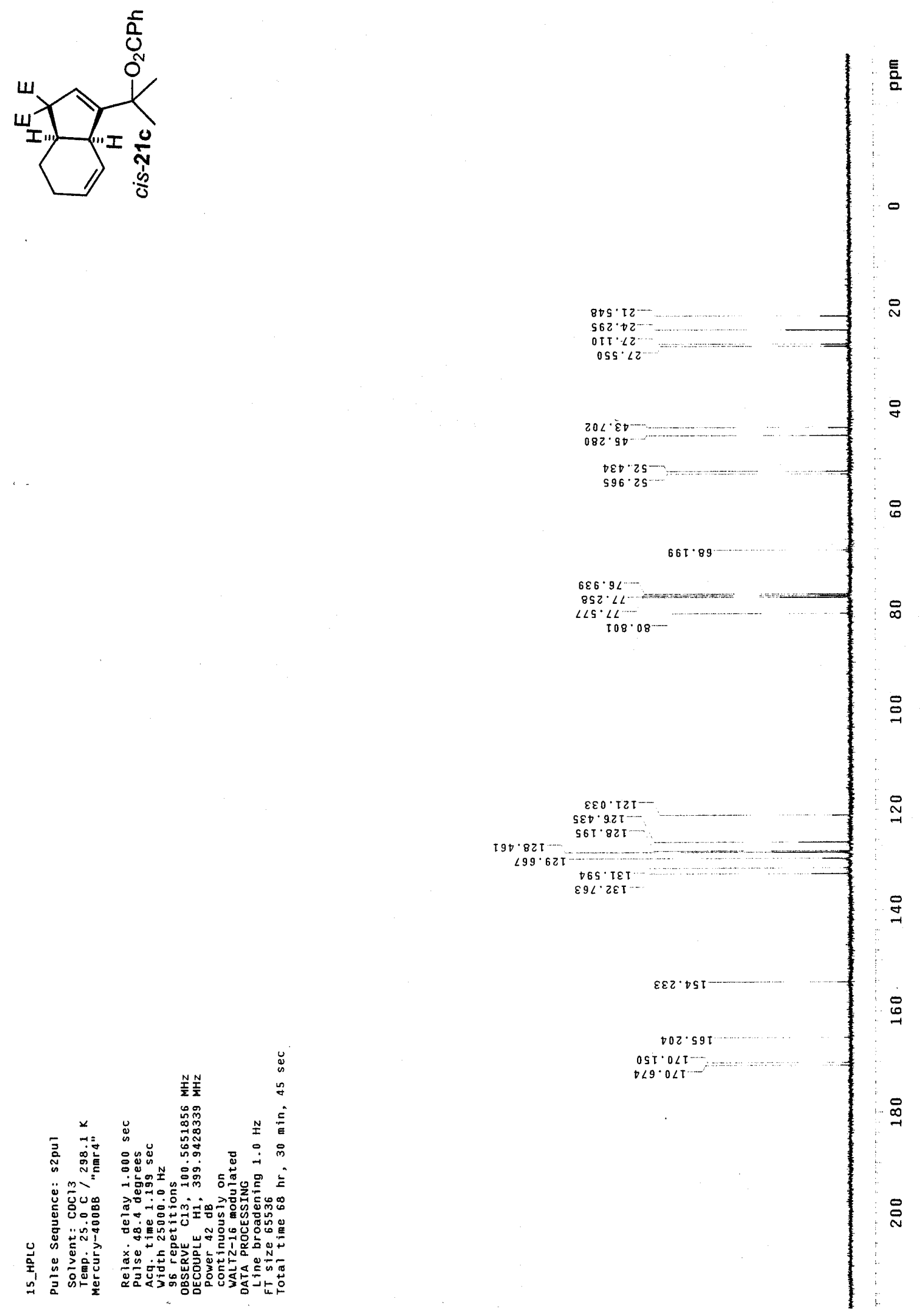


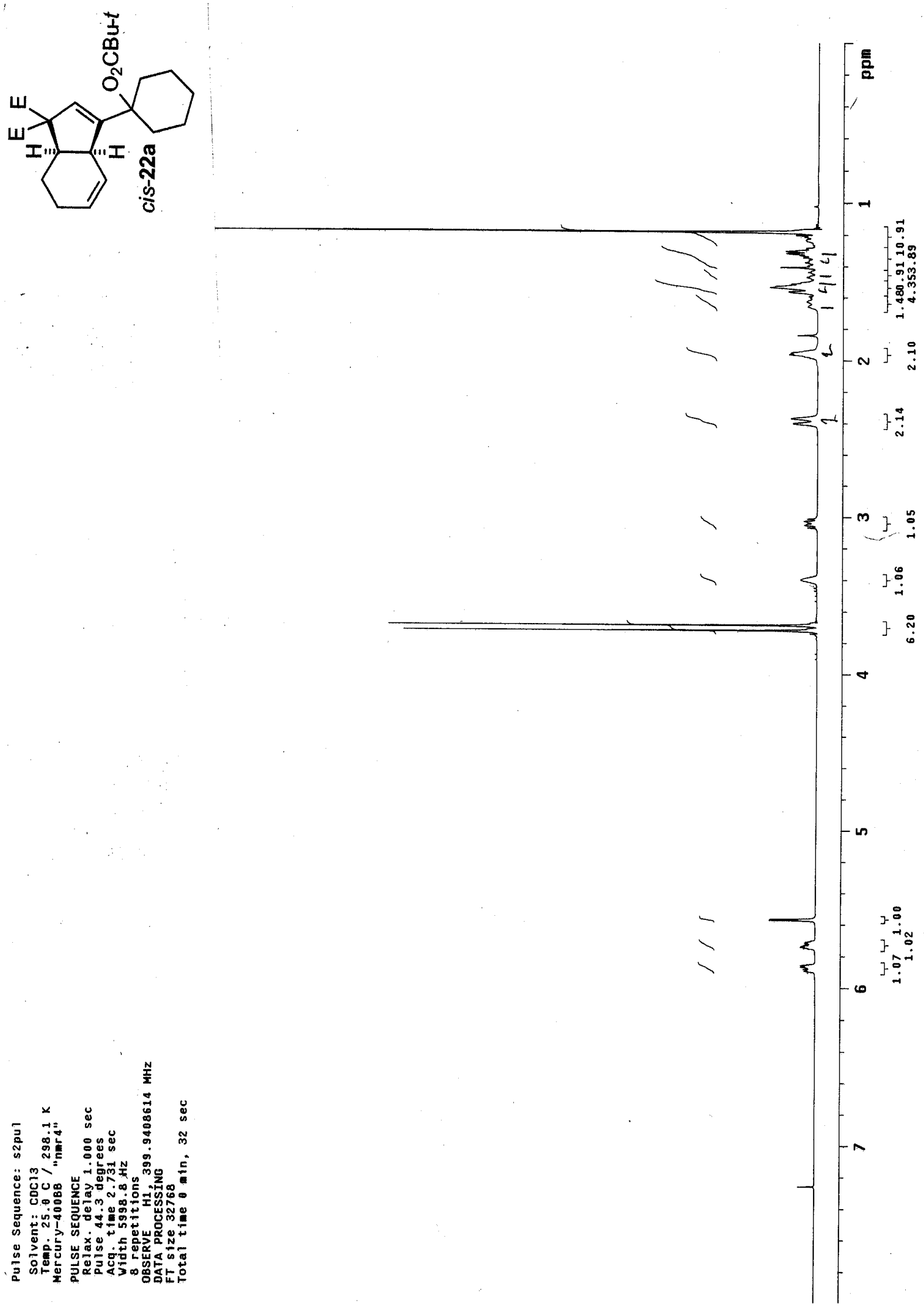



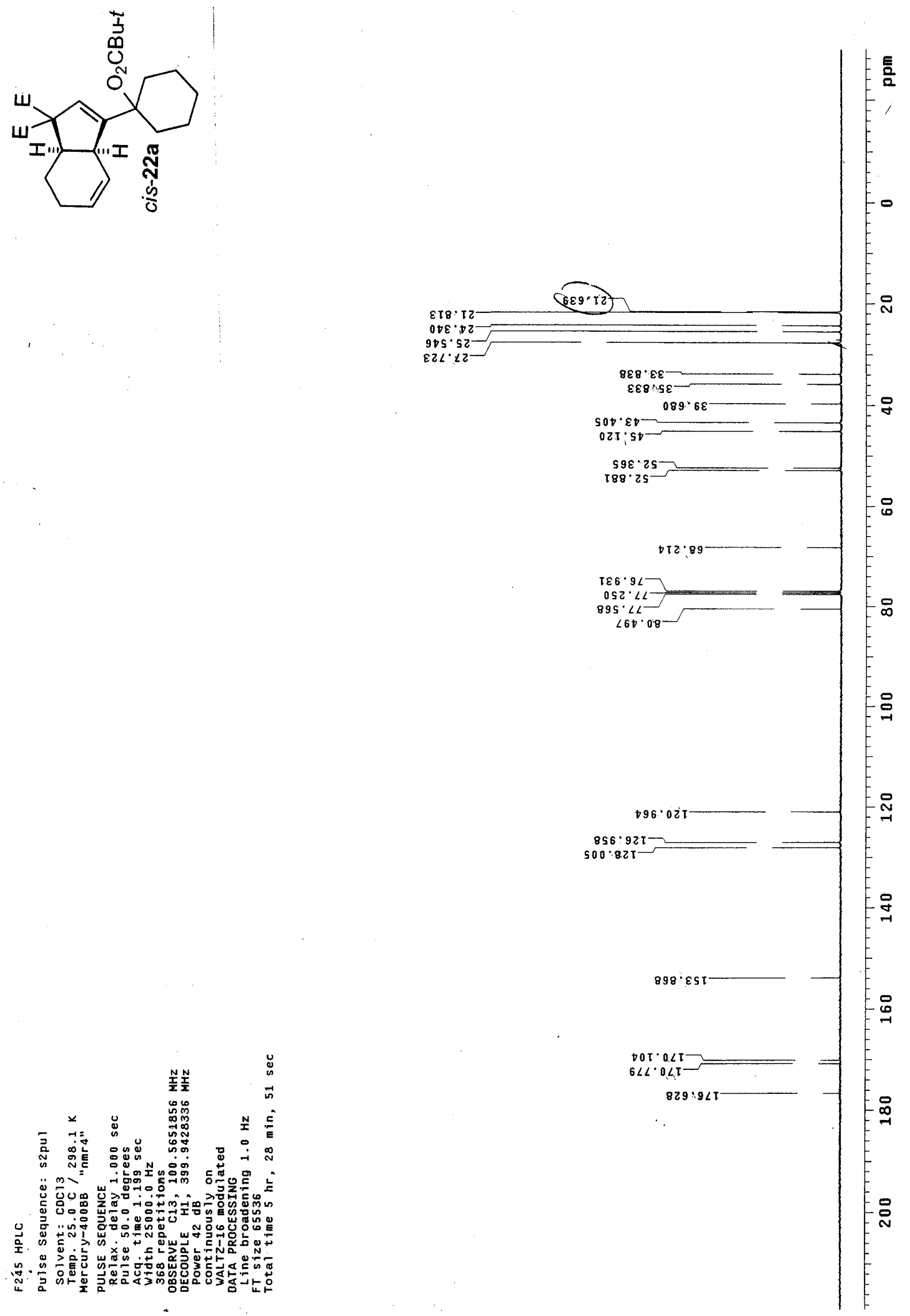
S80
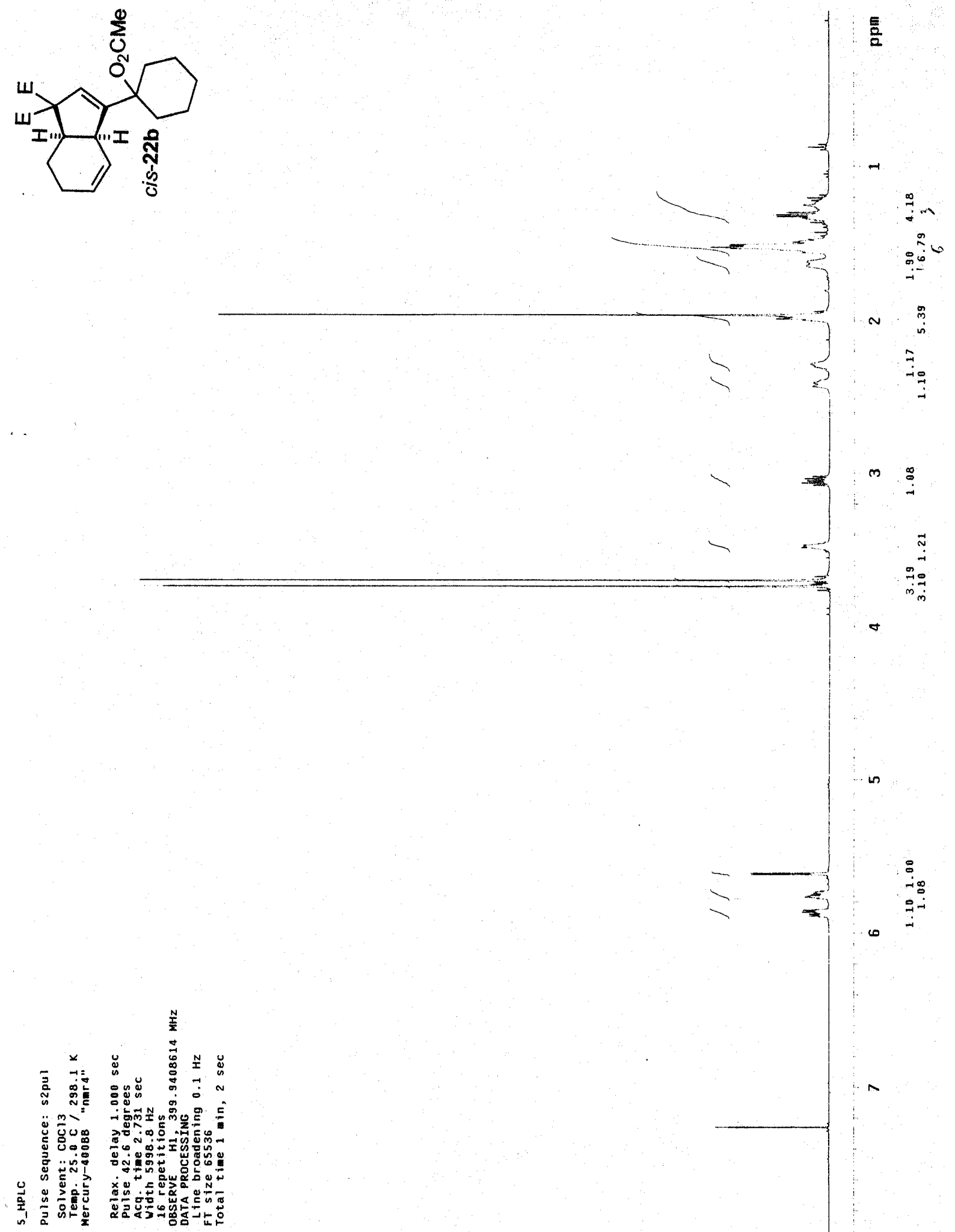

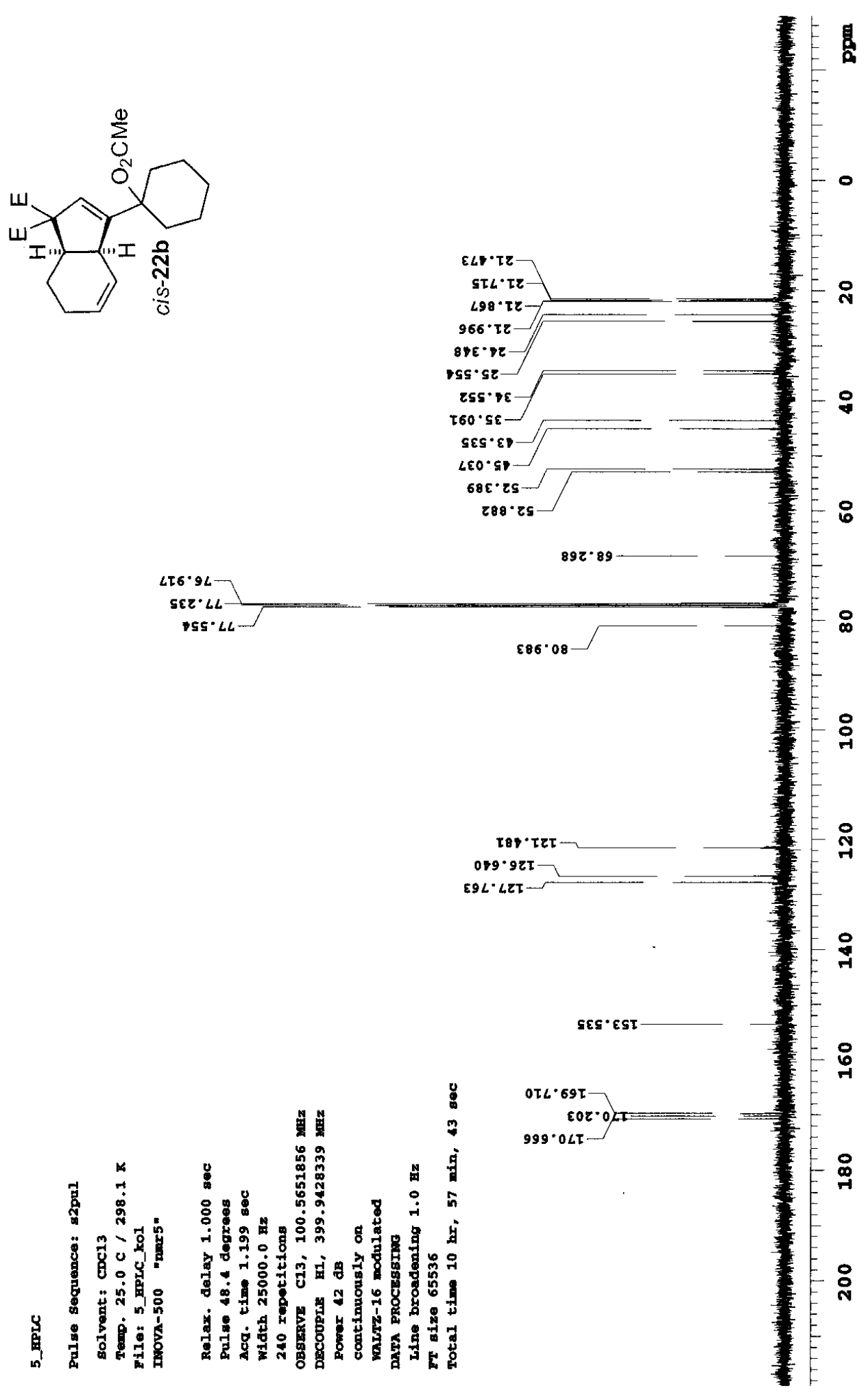

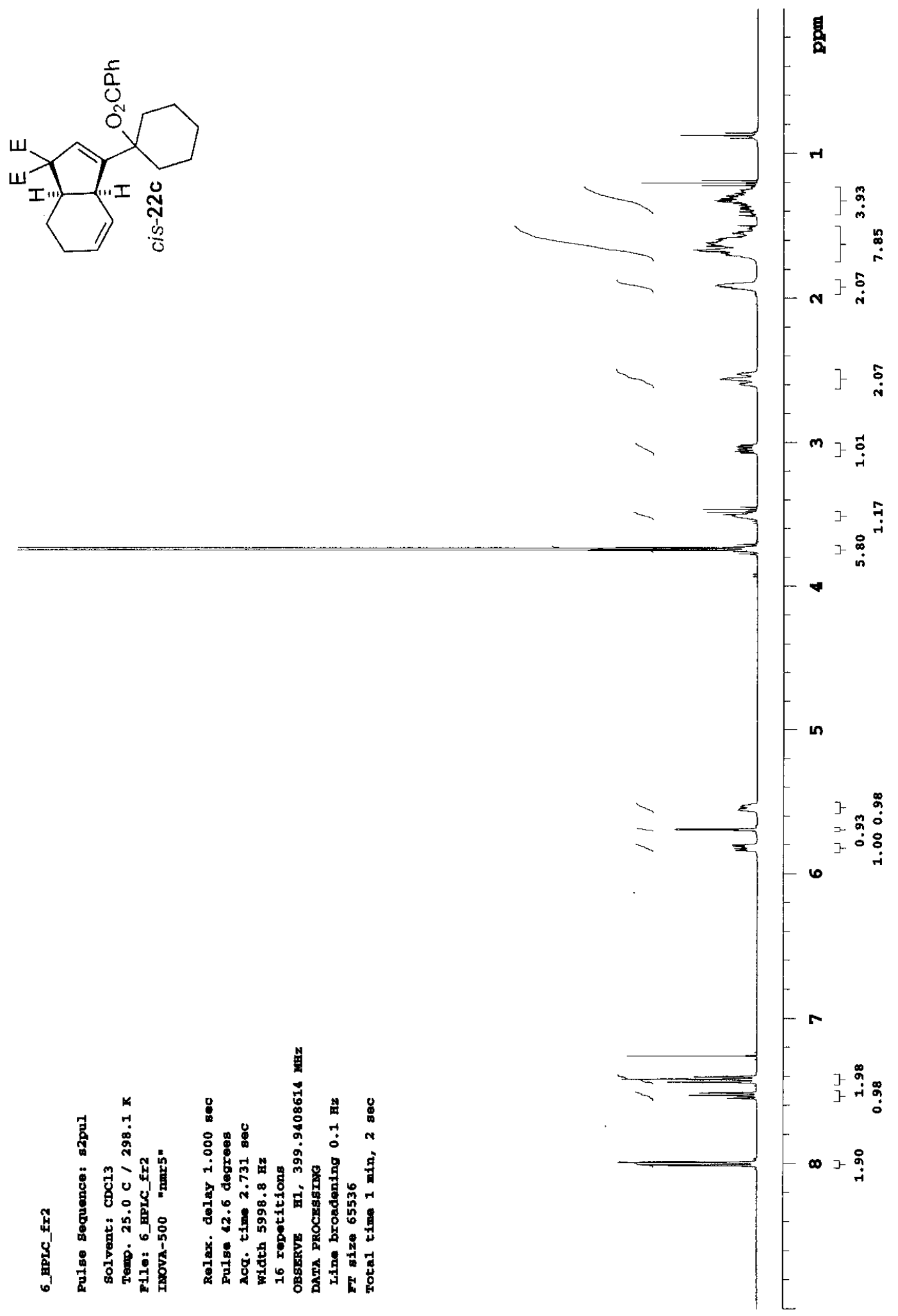

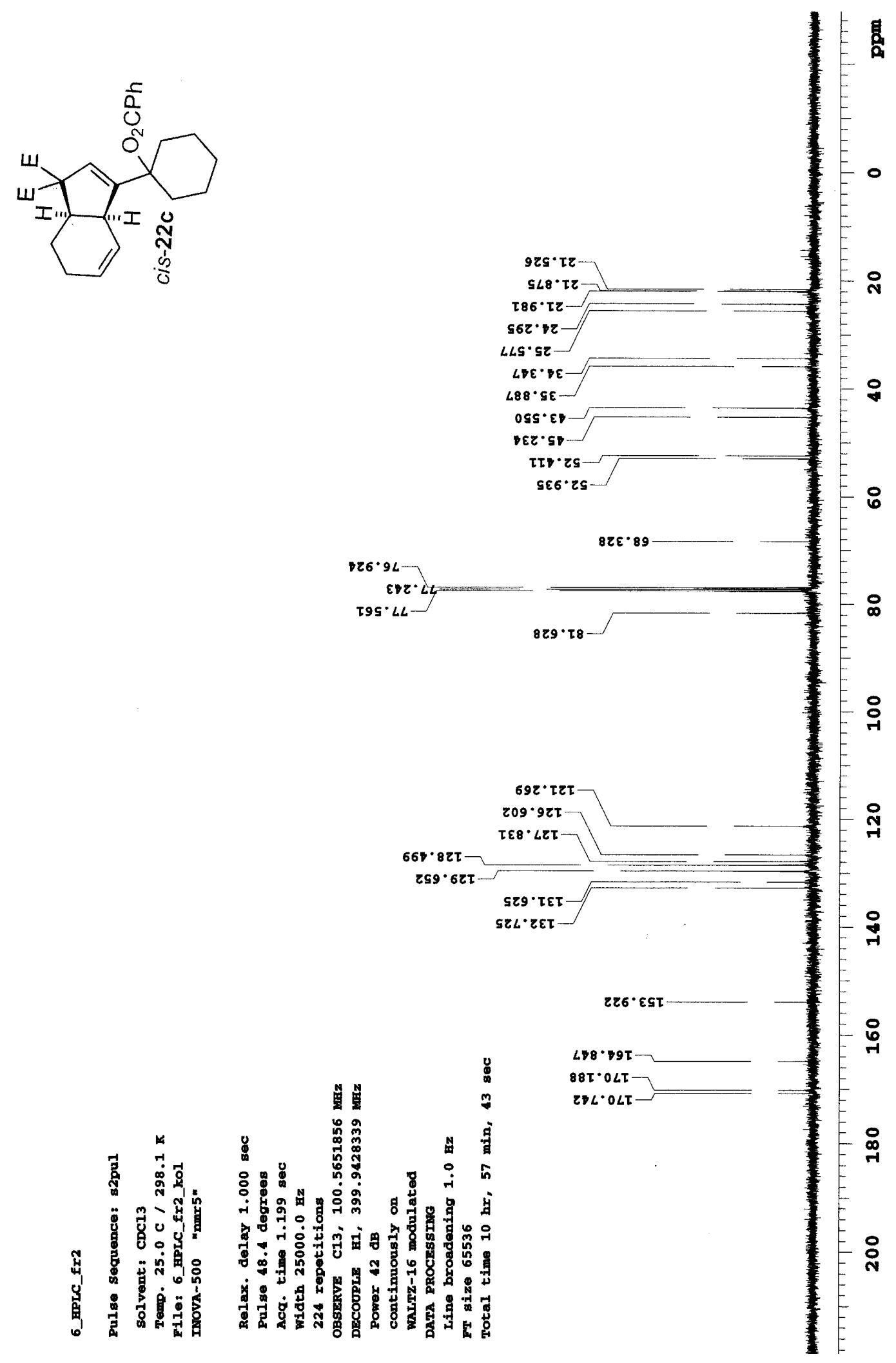

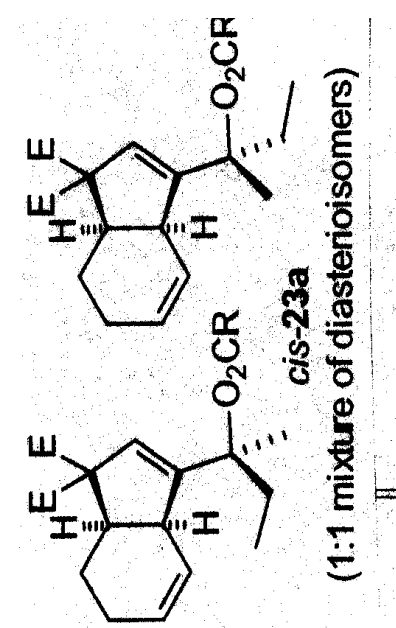

ह
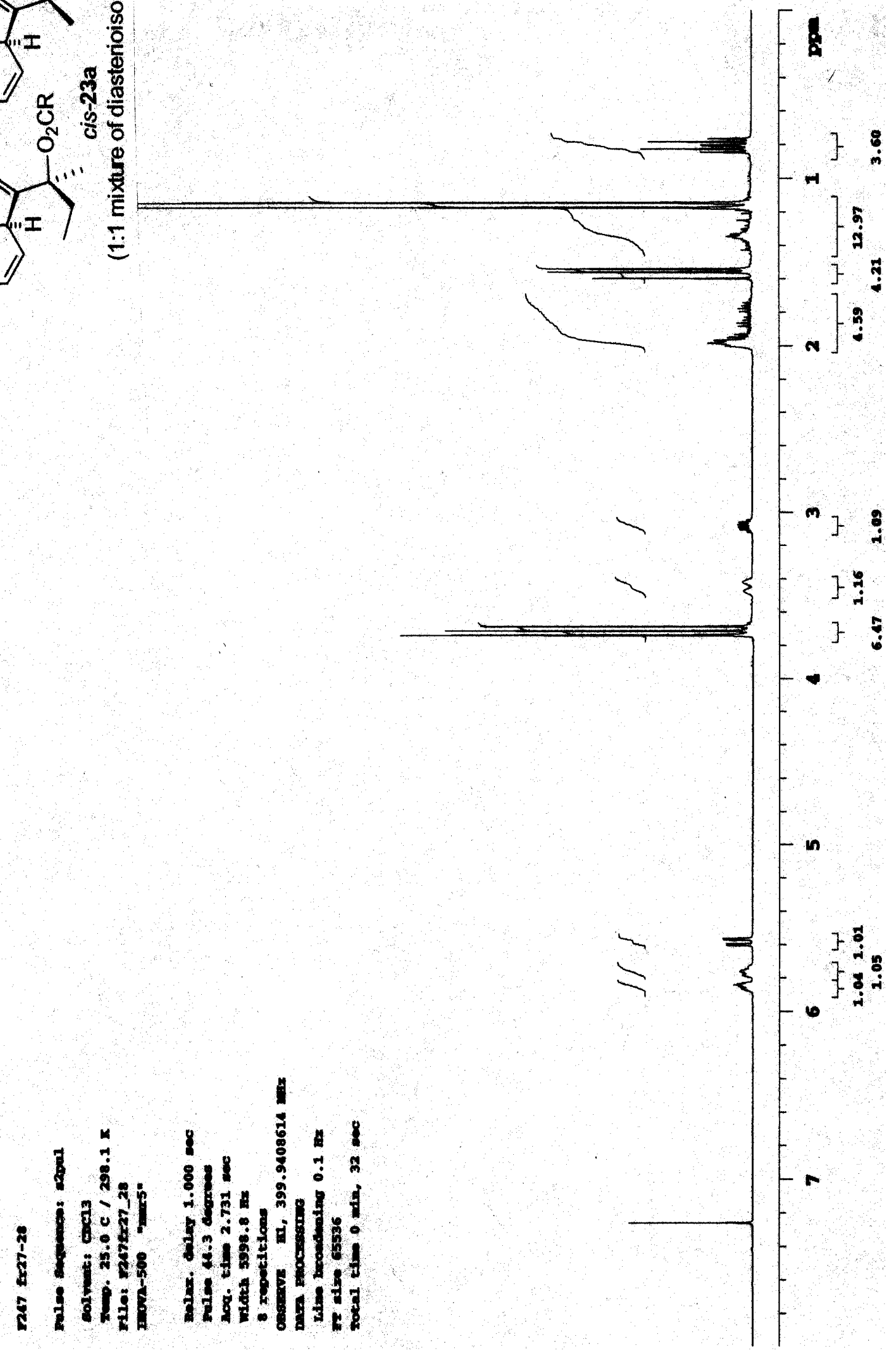

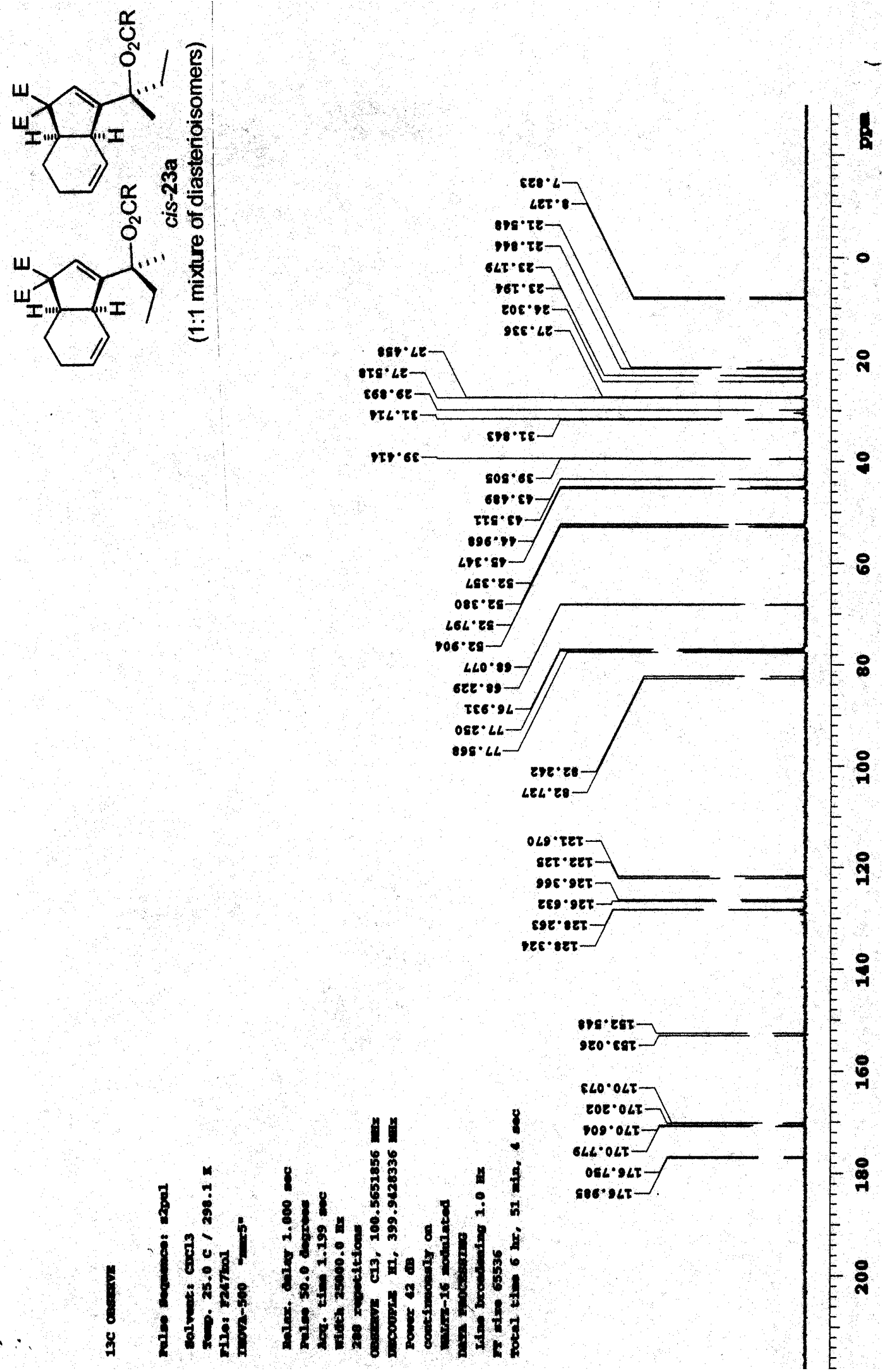

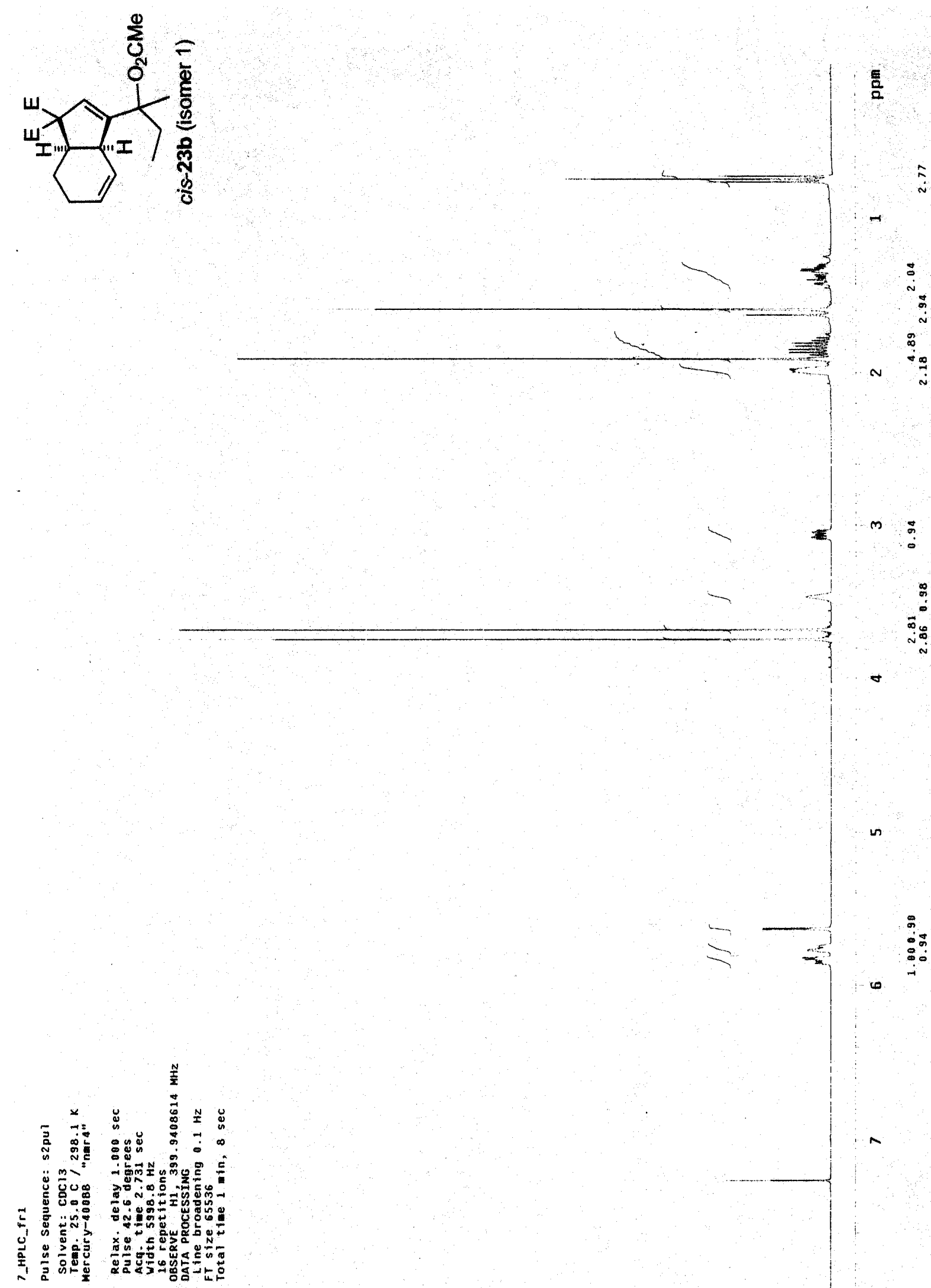

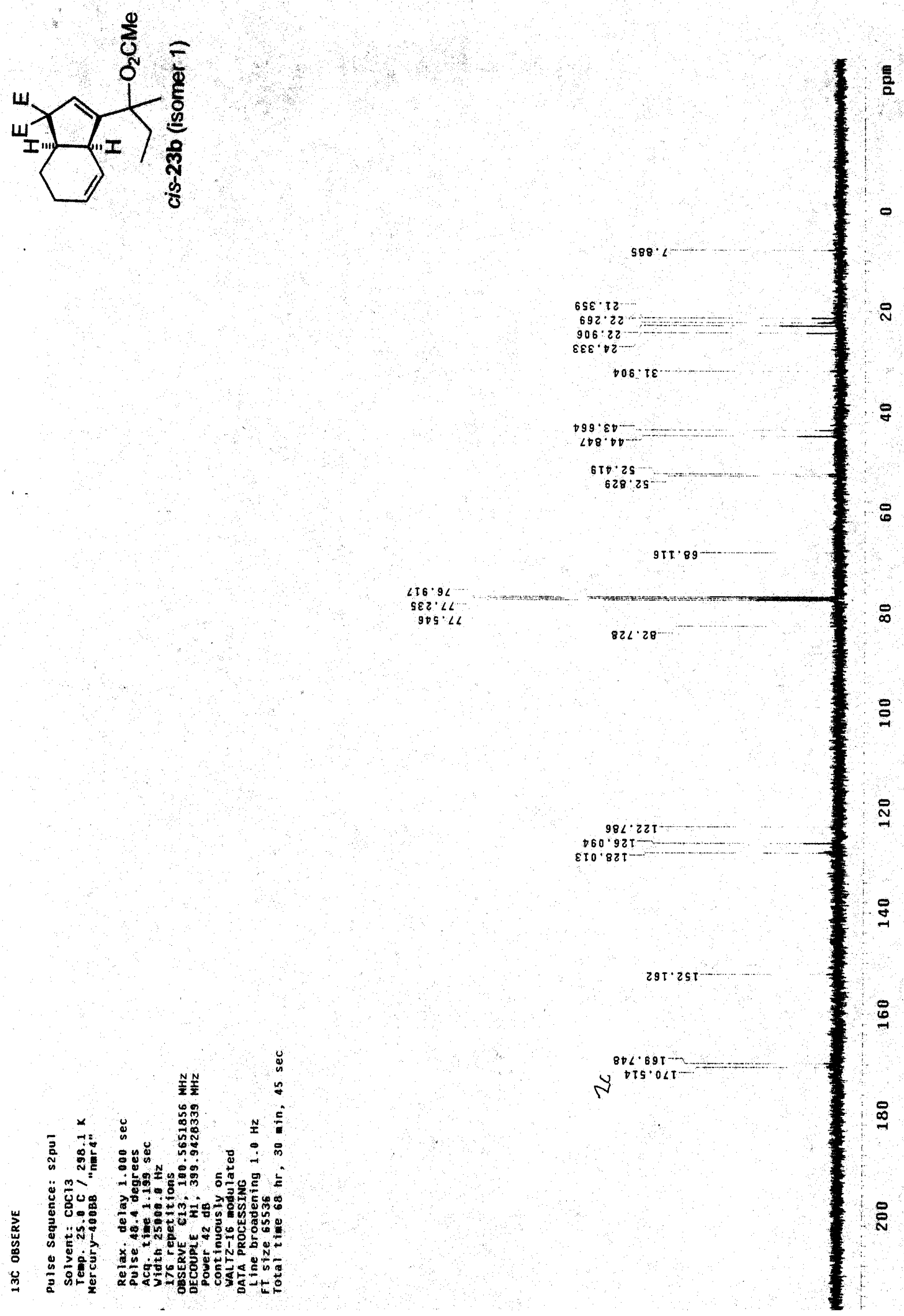

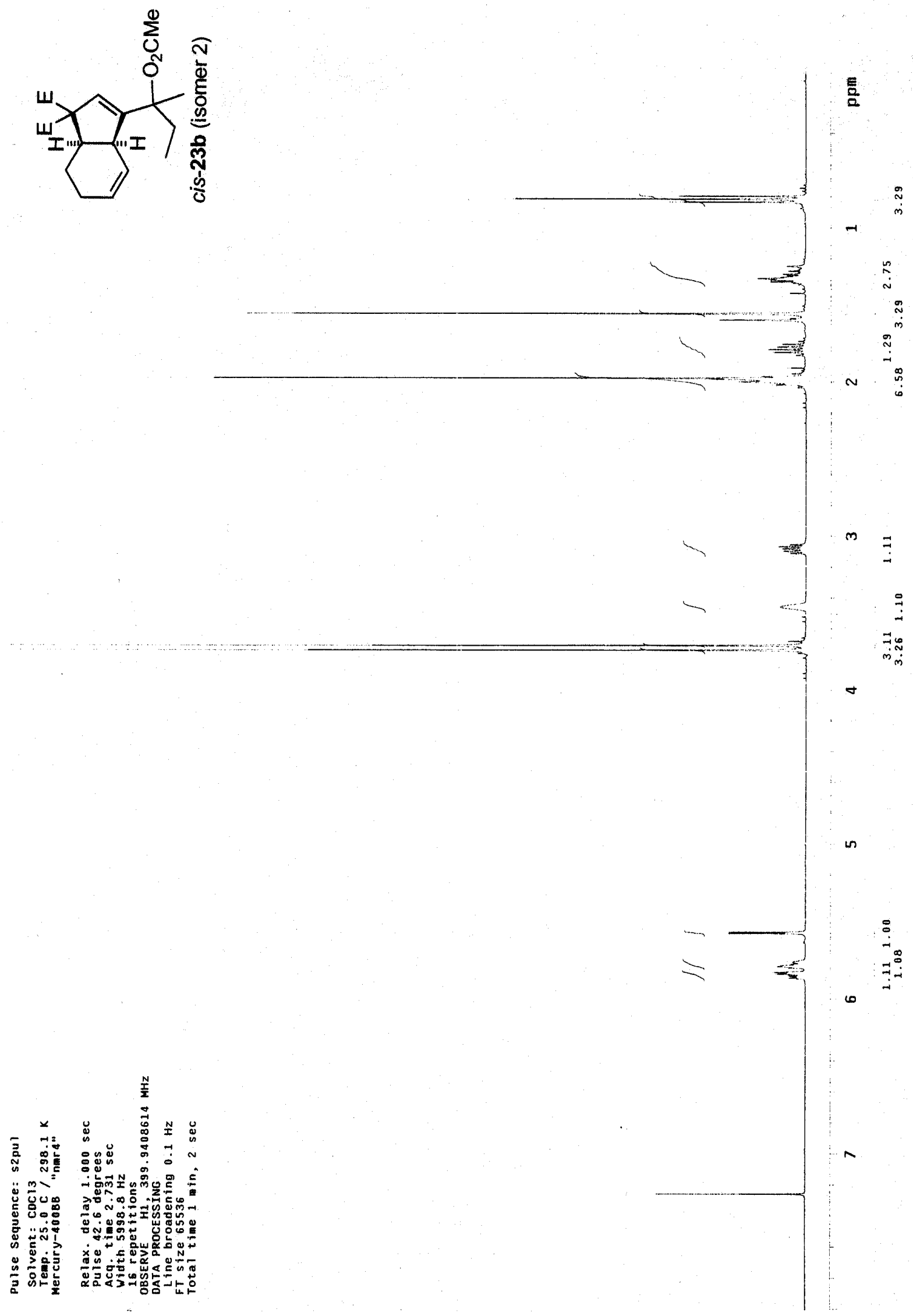

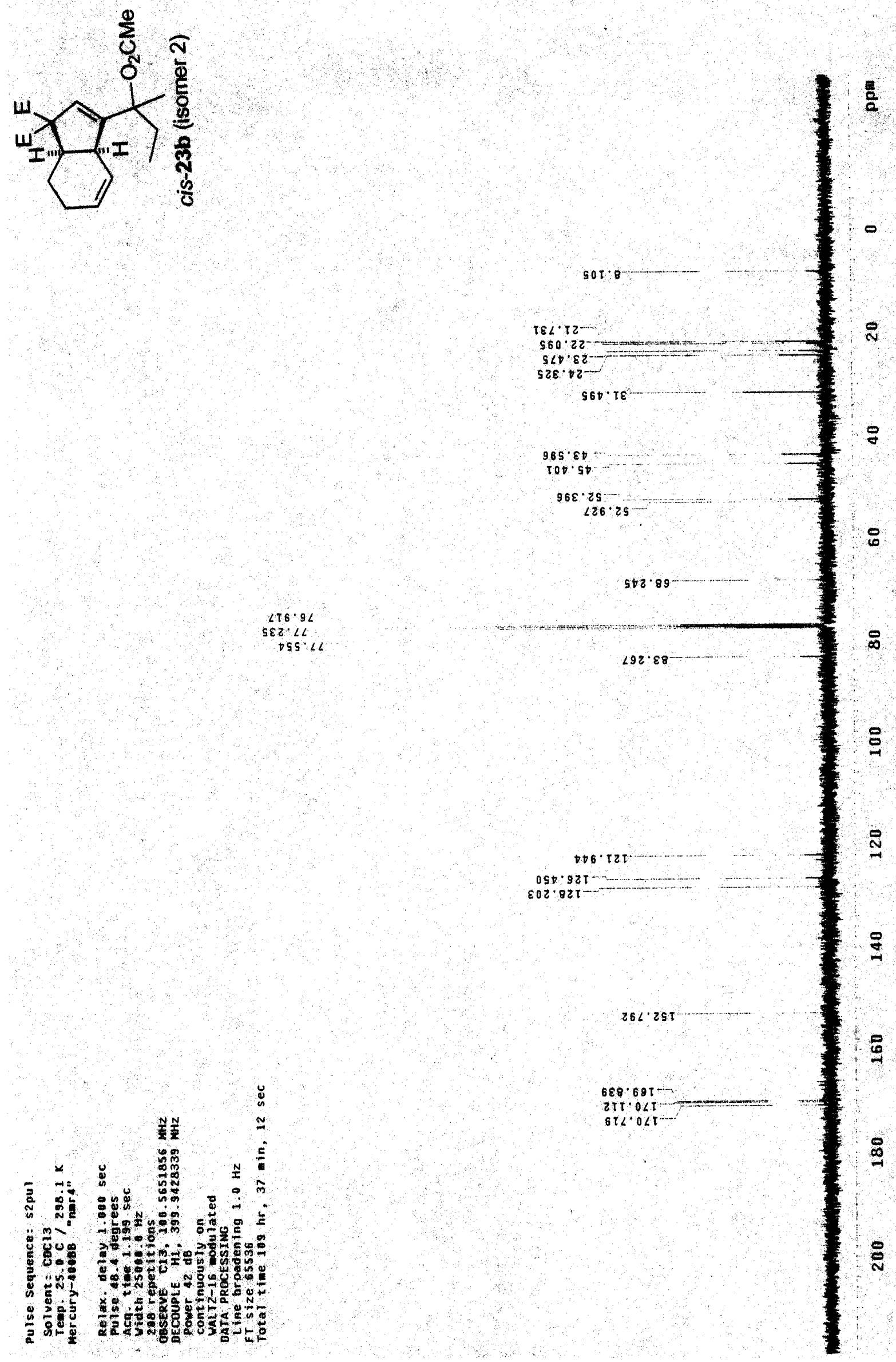

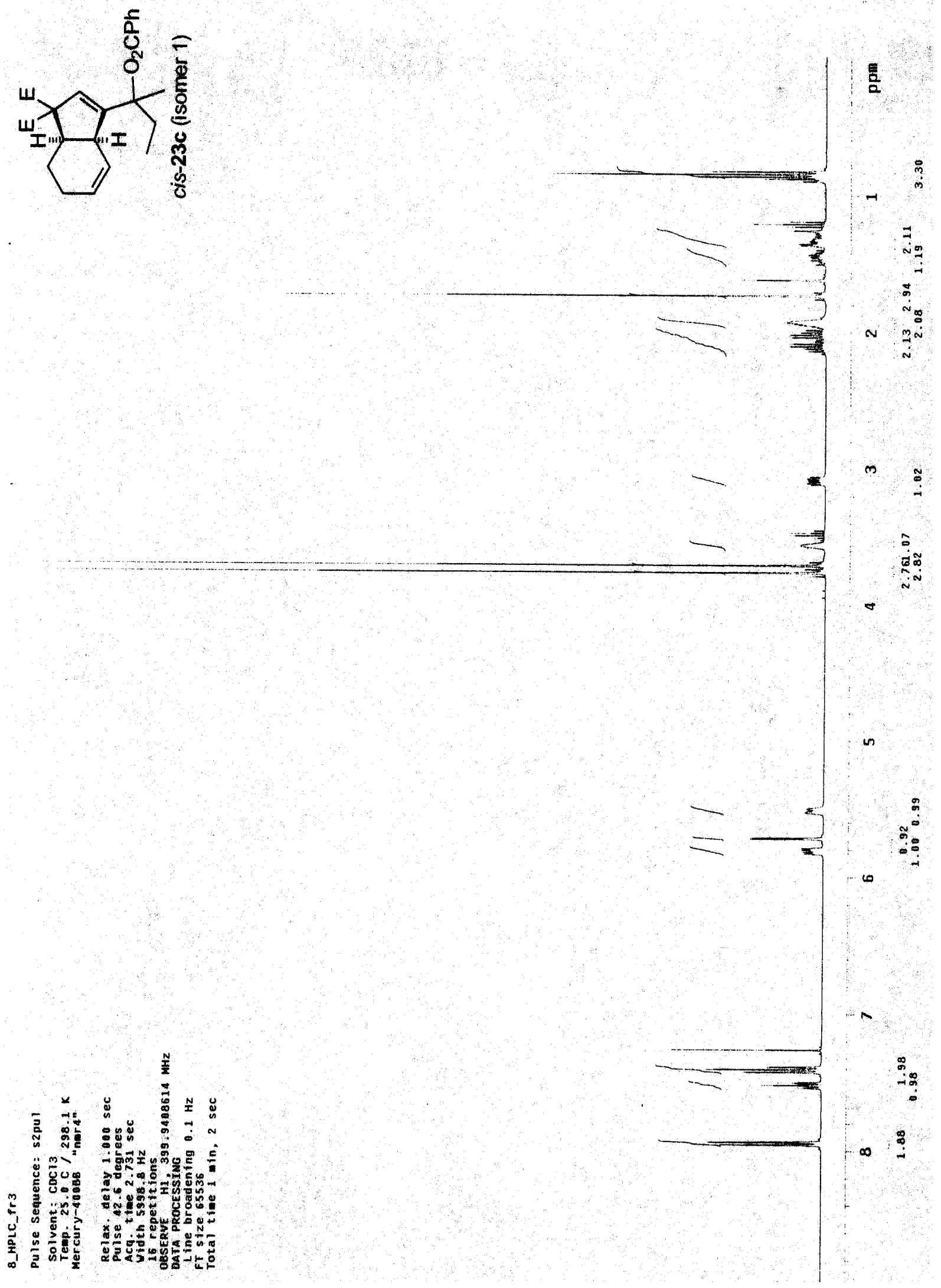

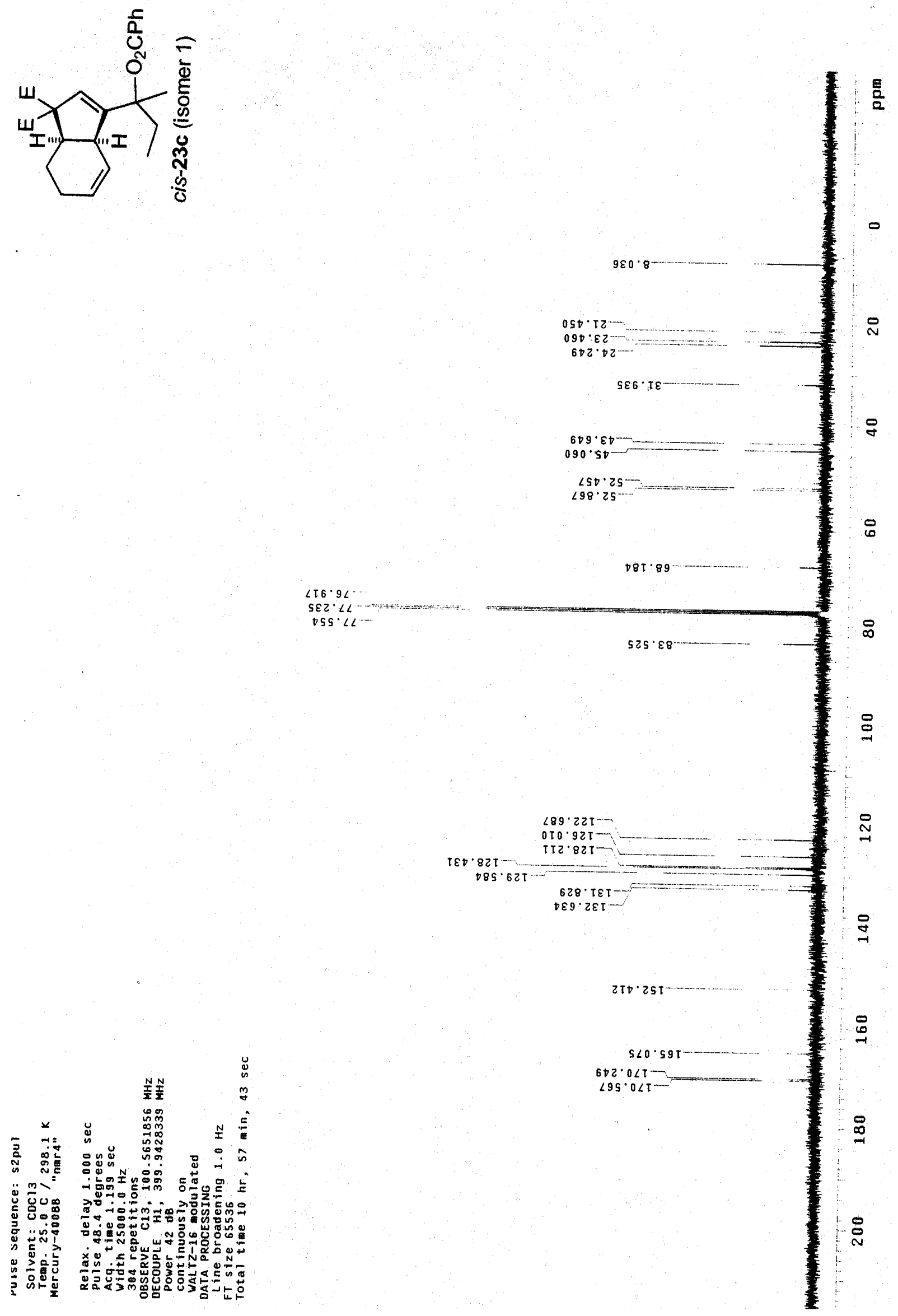

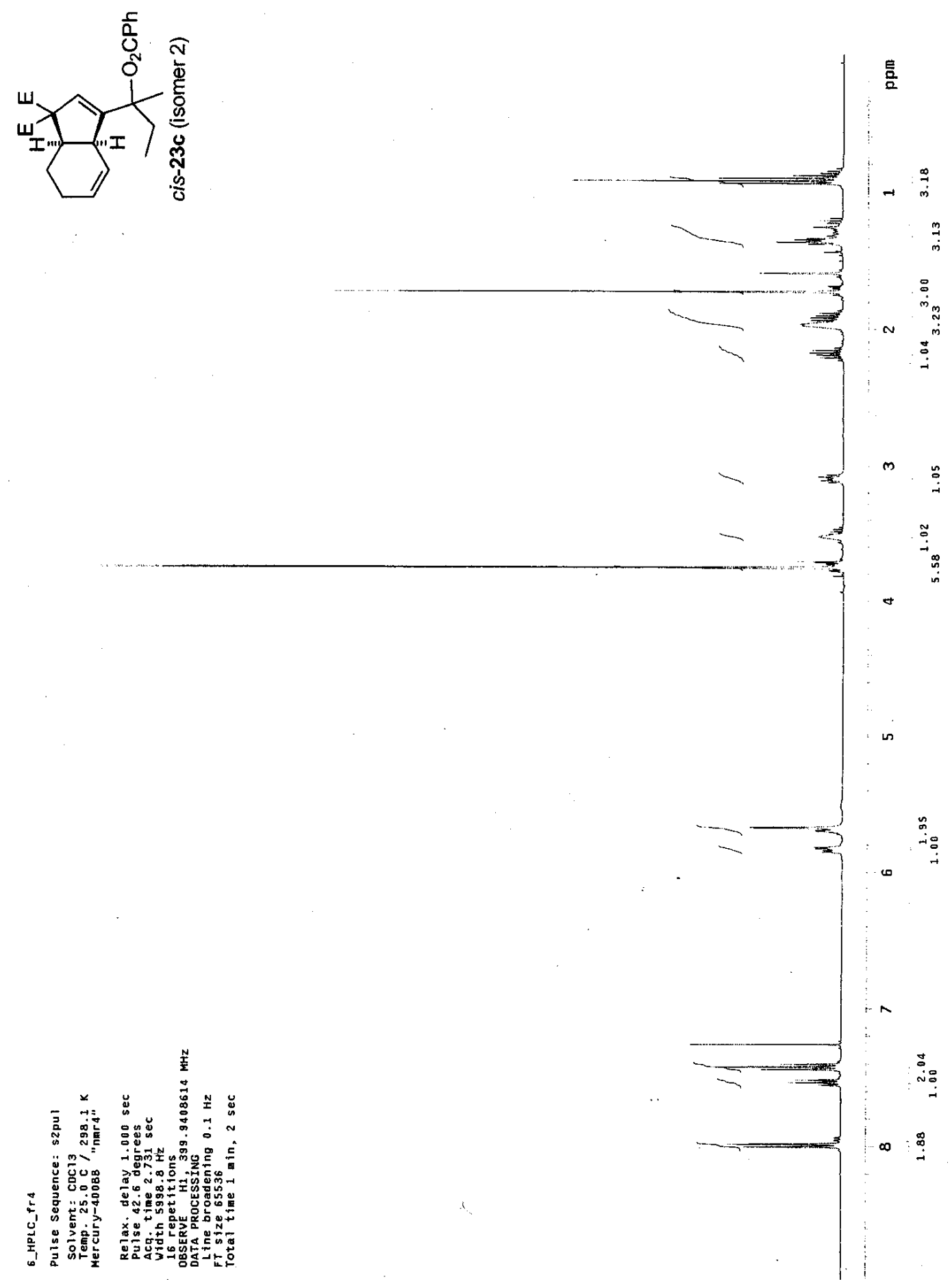

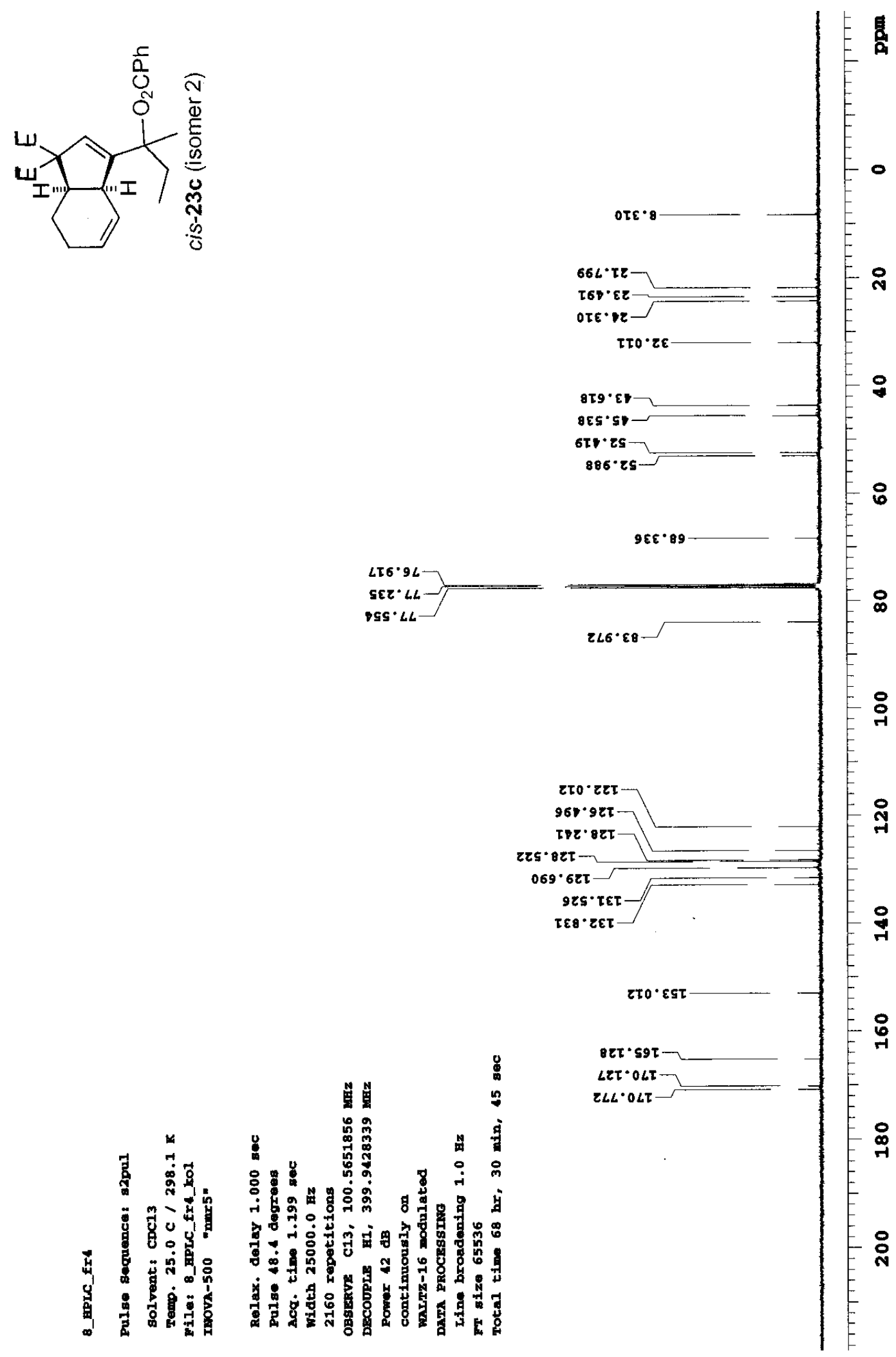

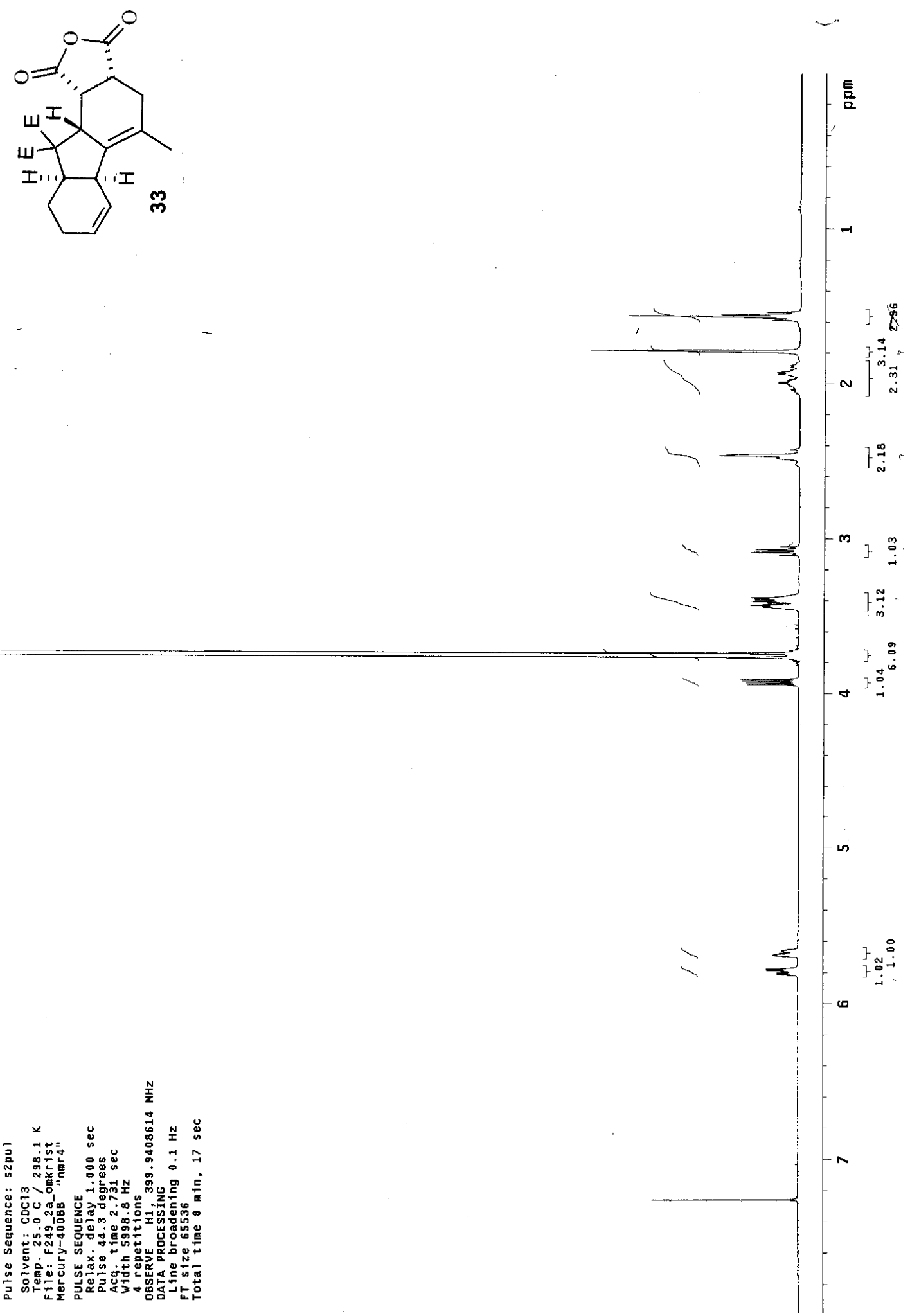

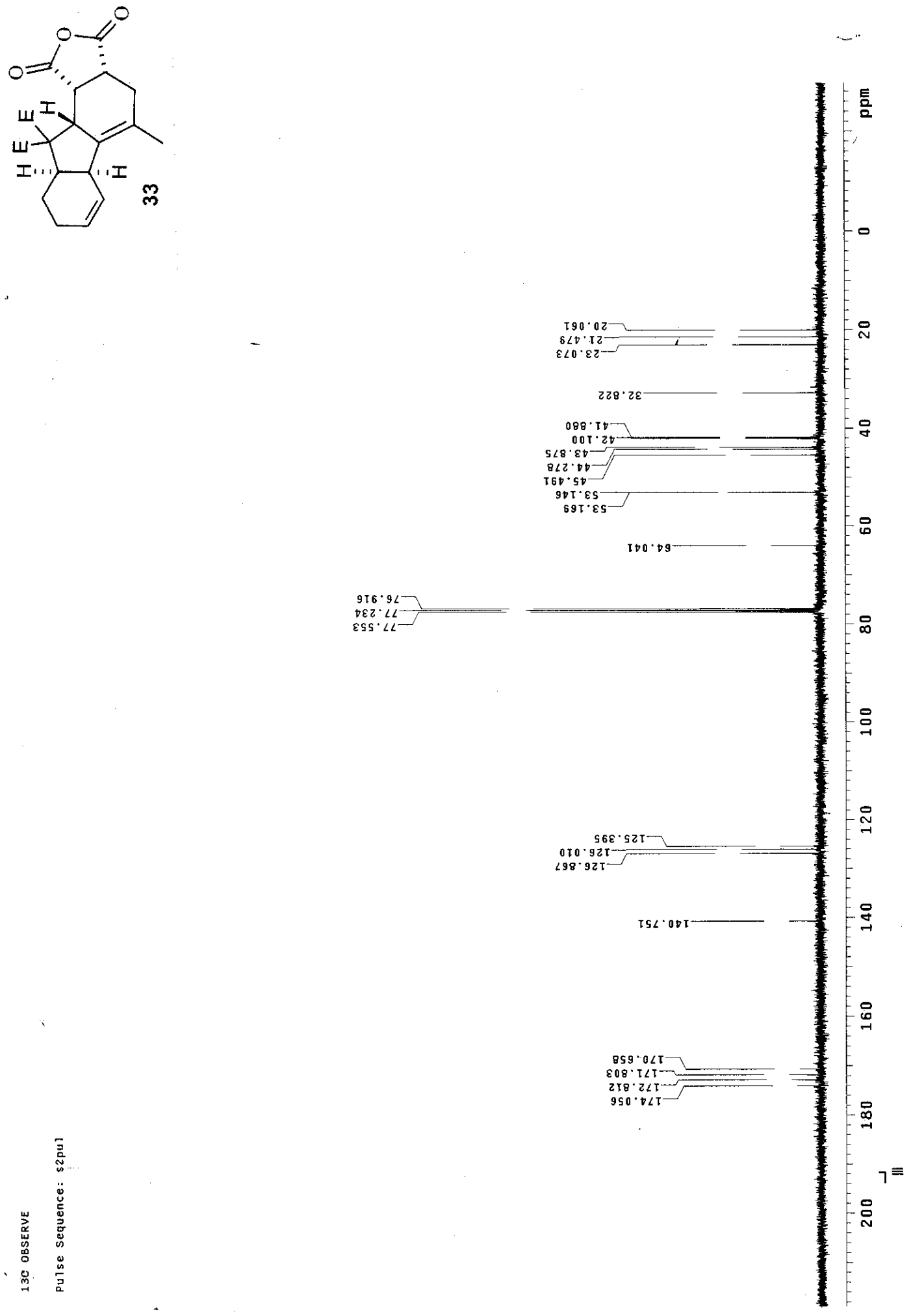

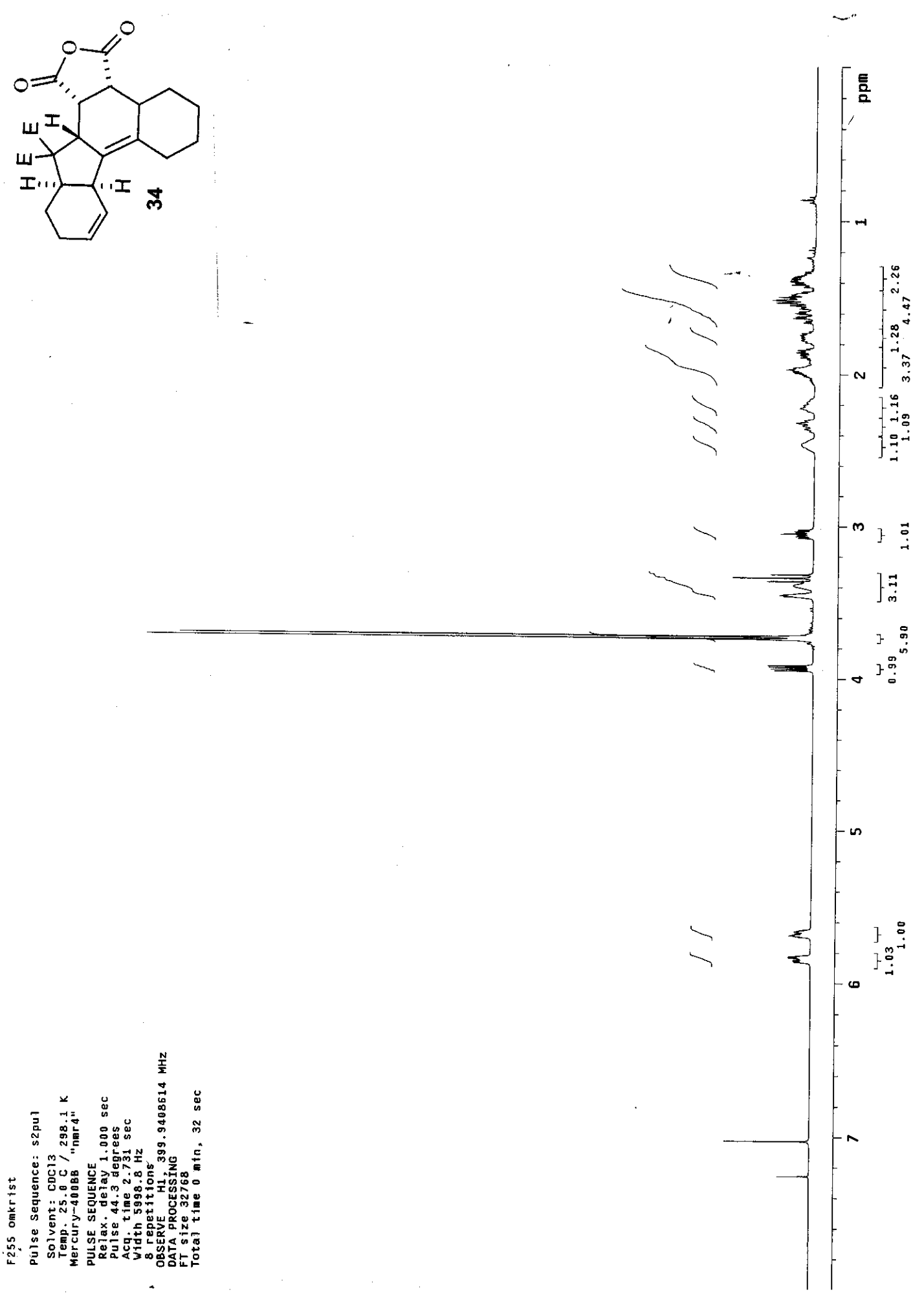

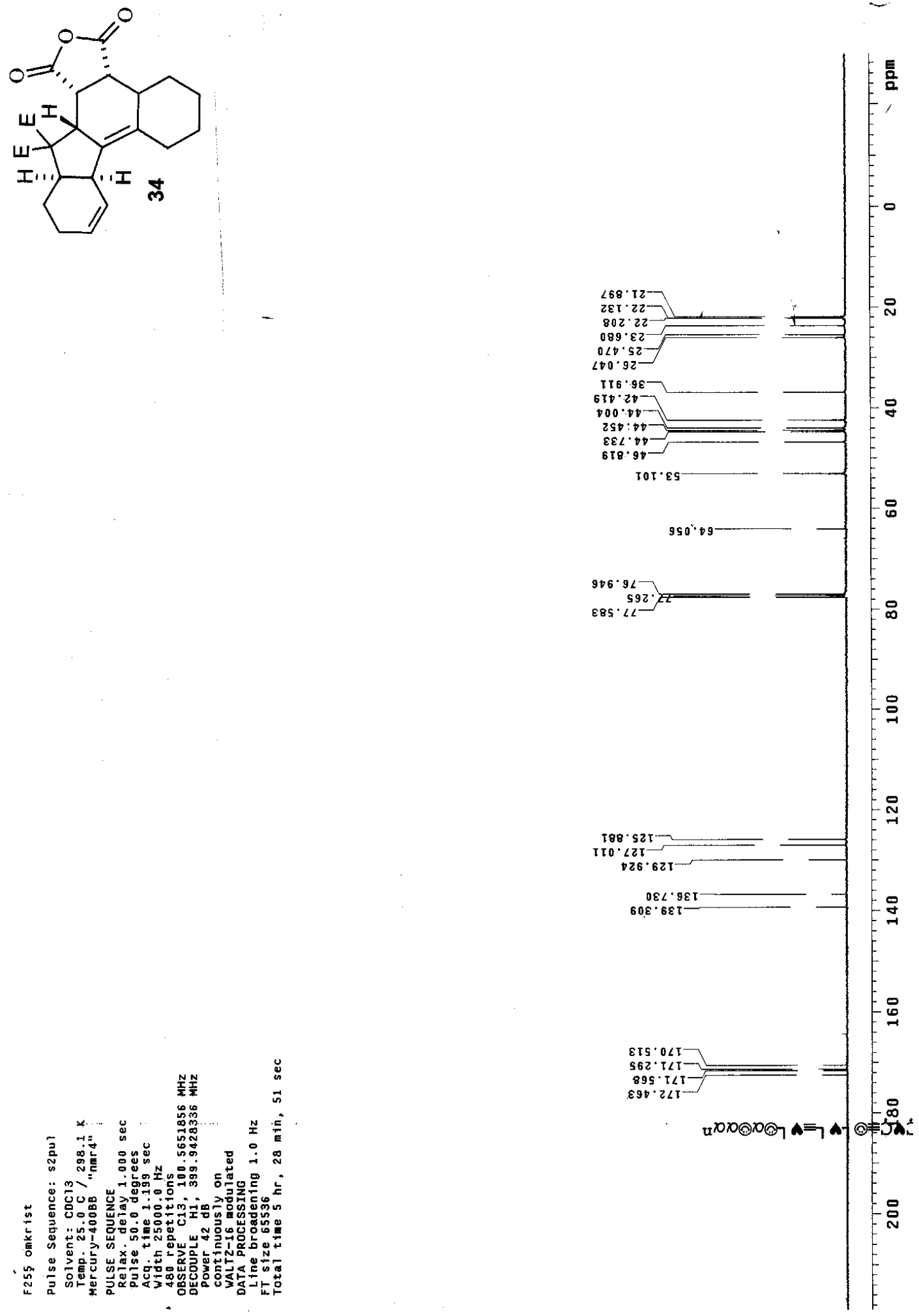

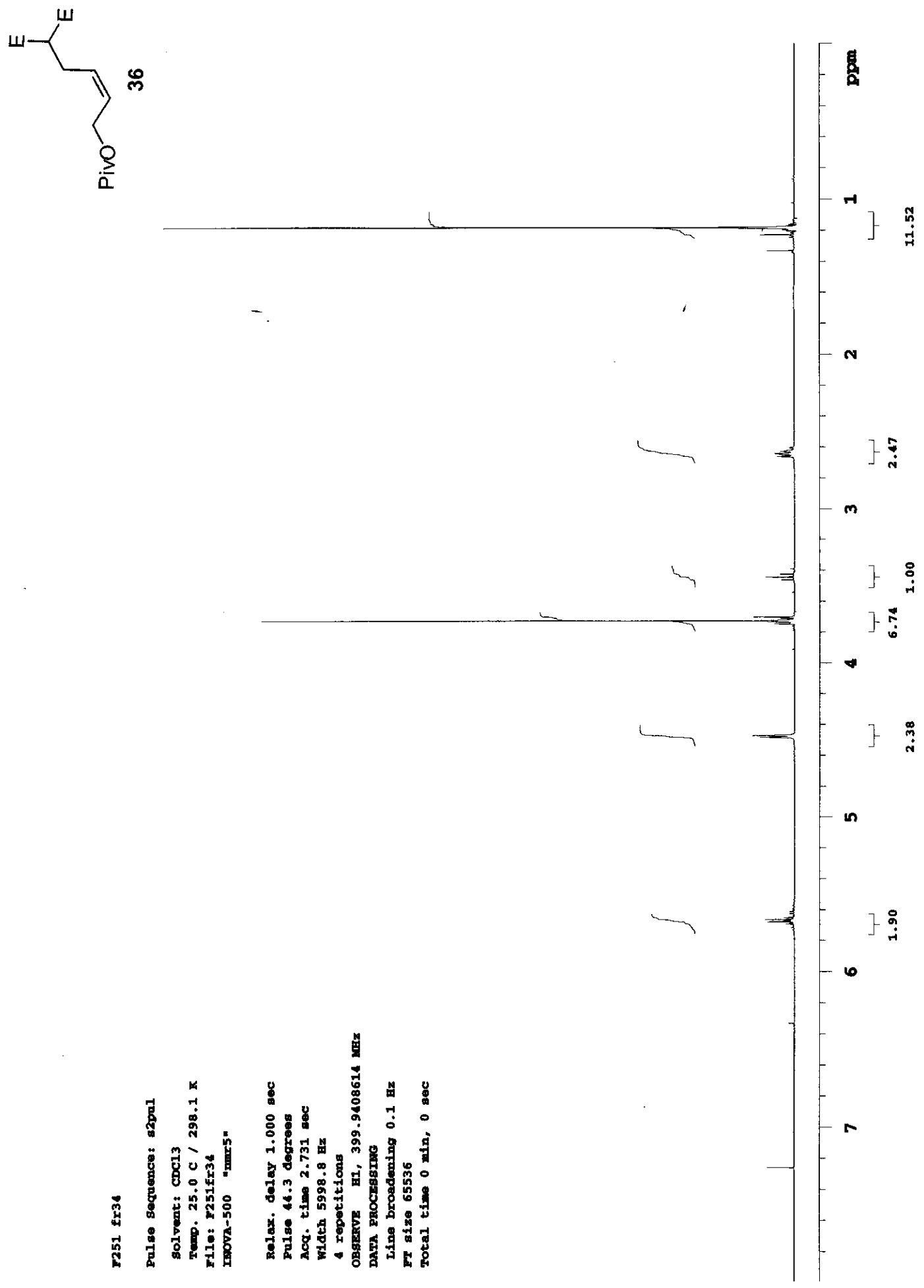

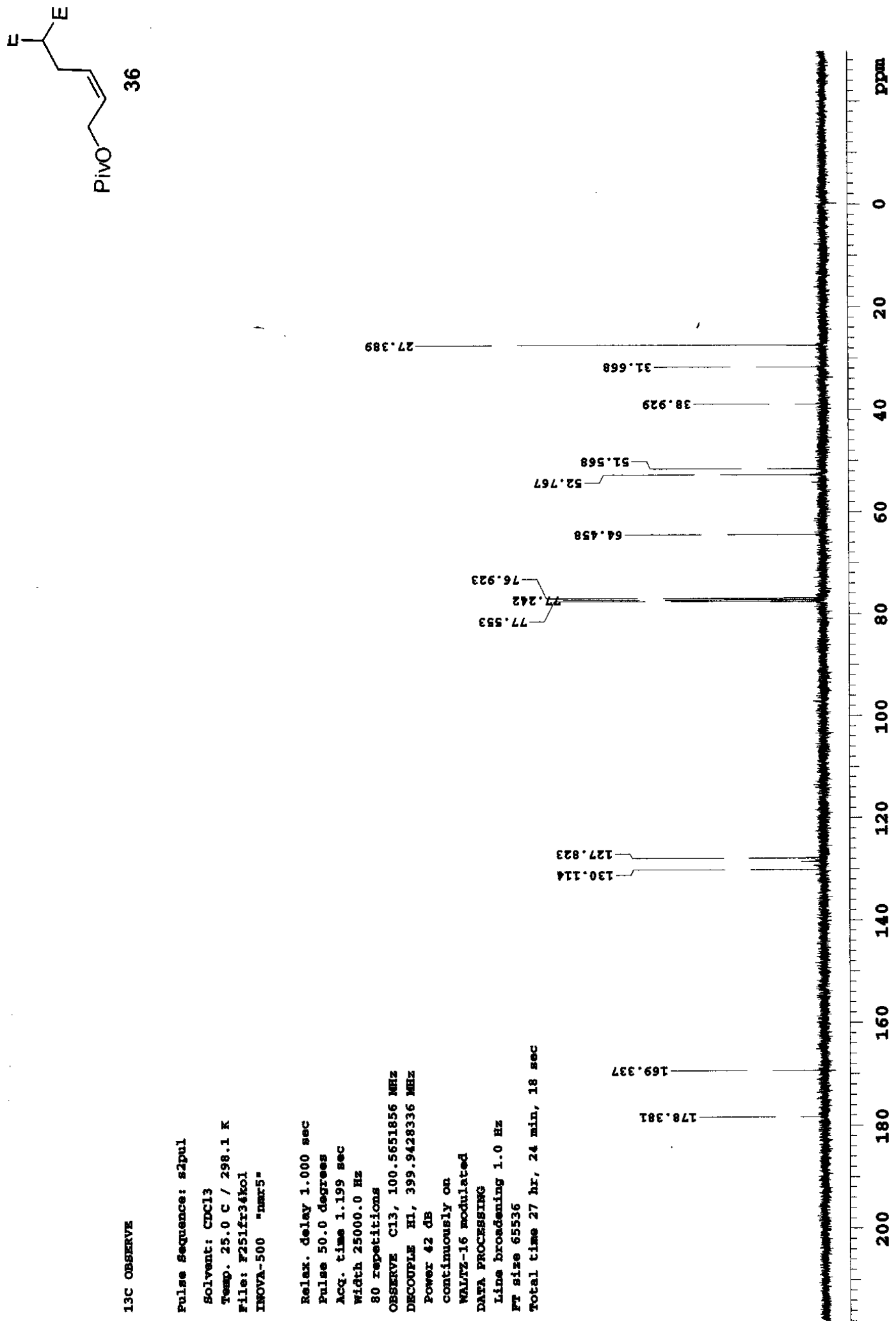


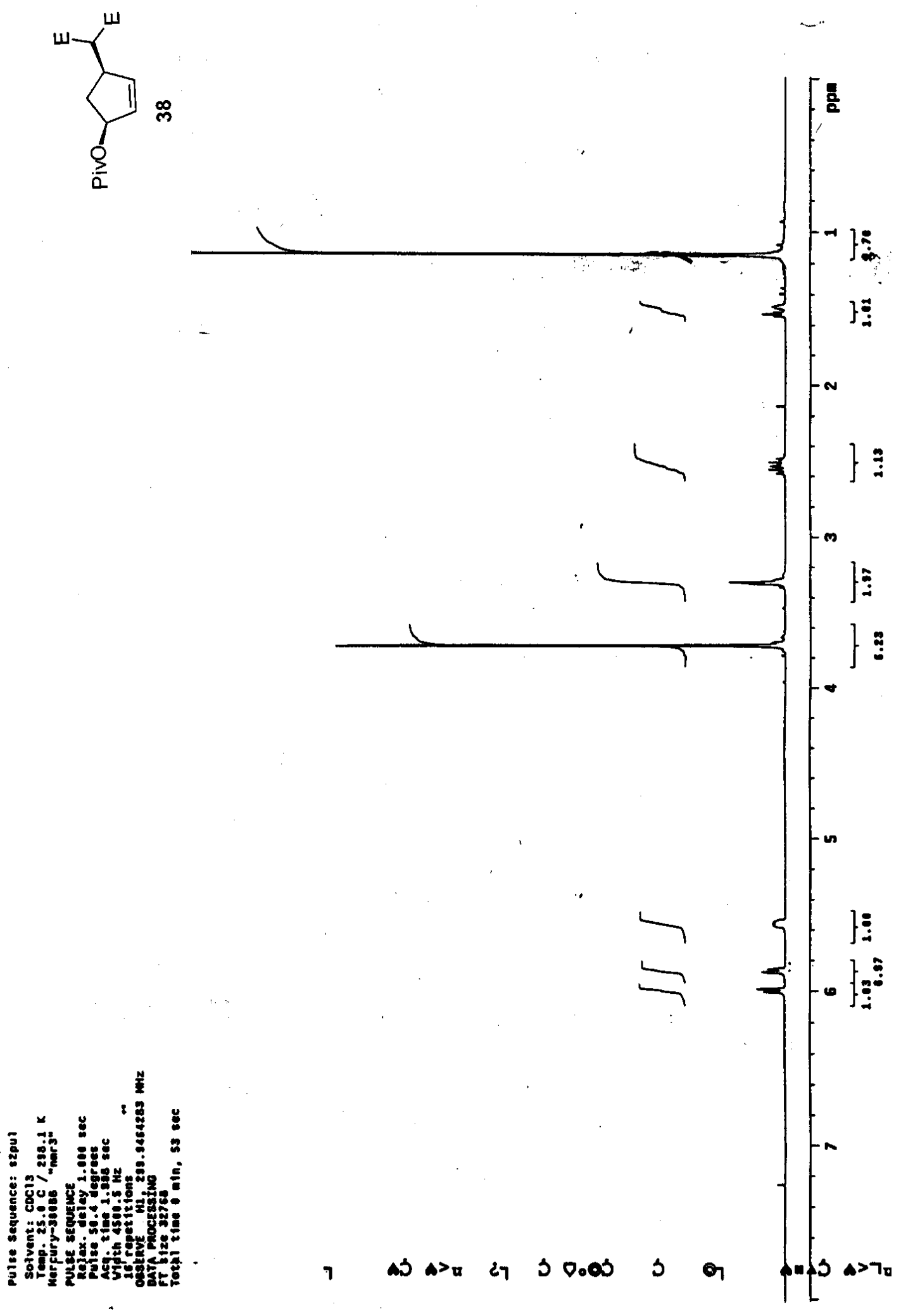




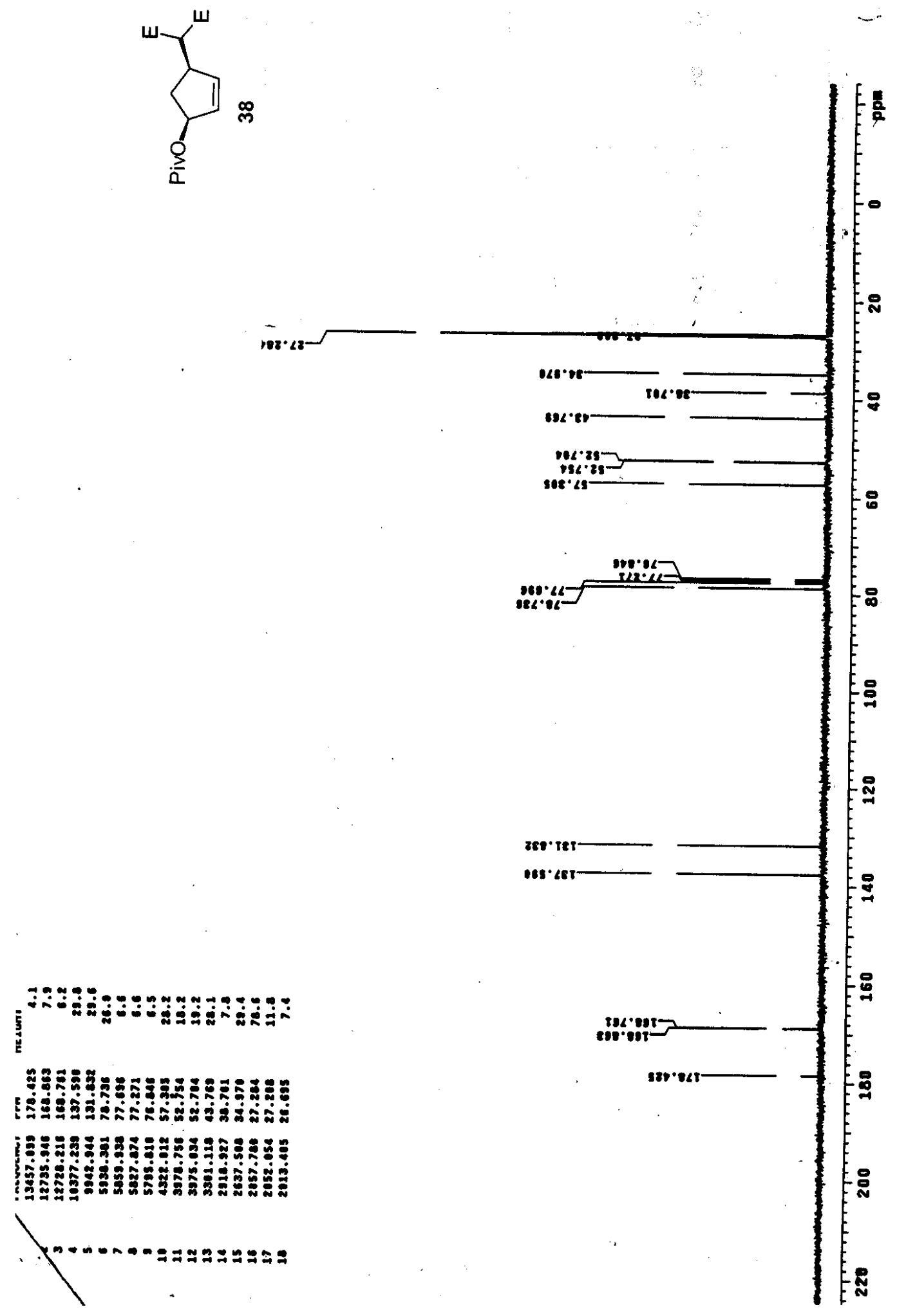



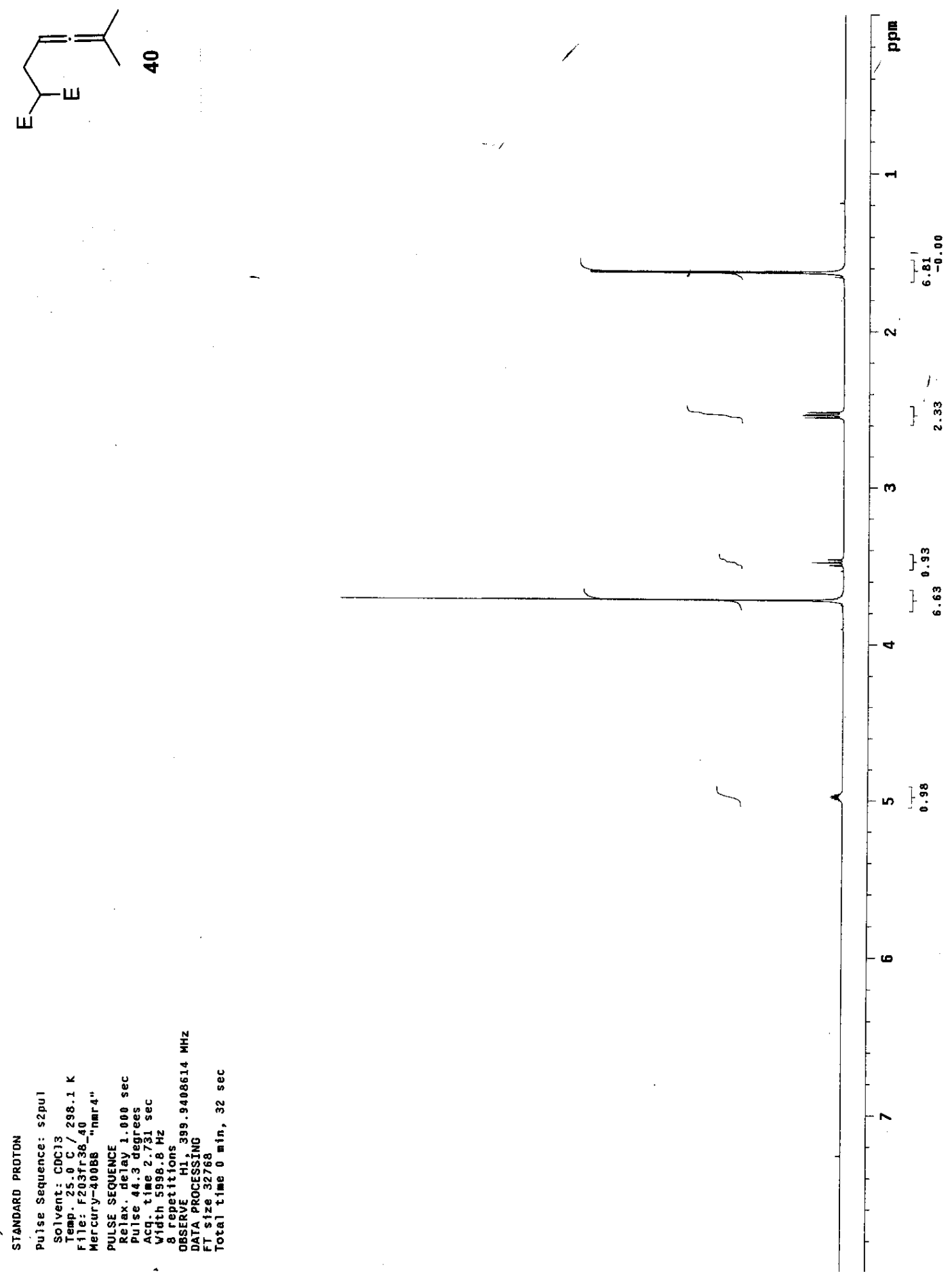

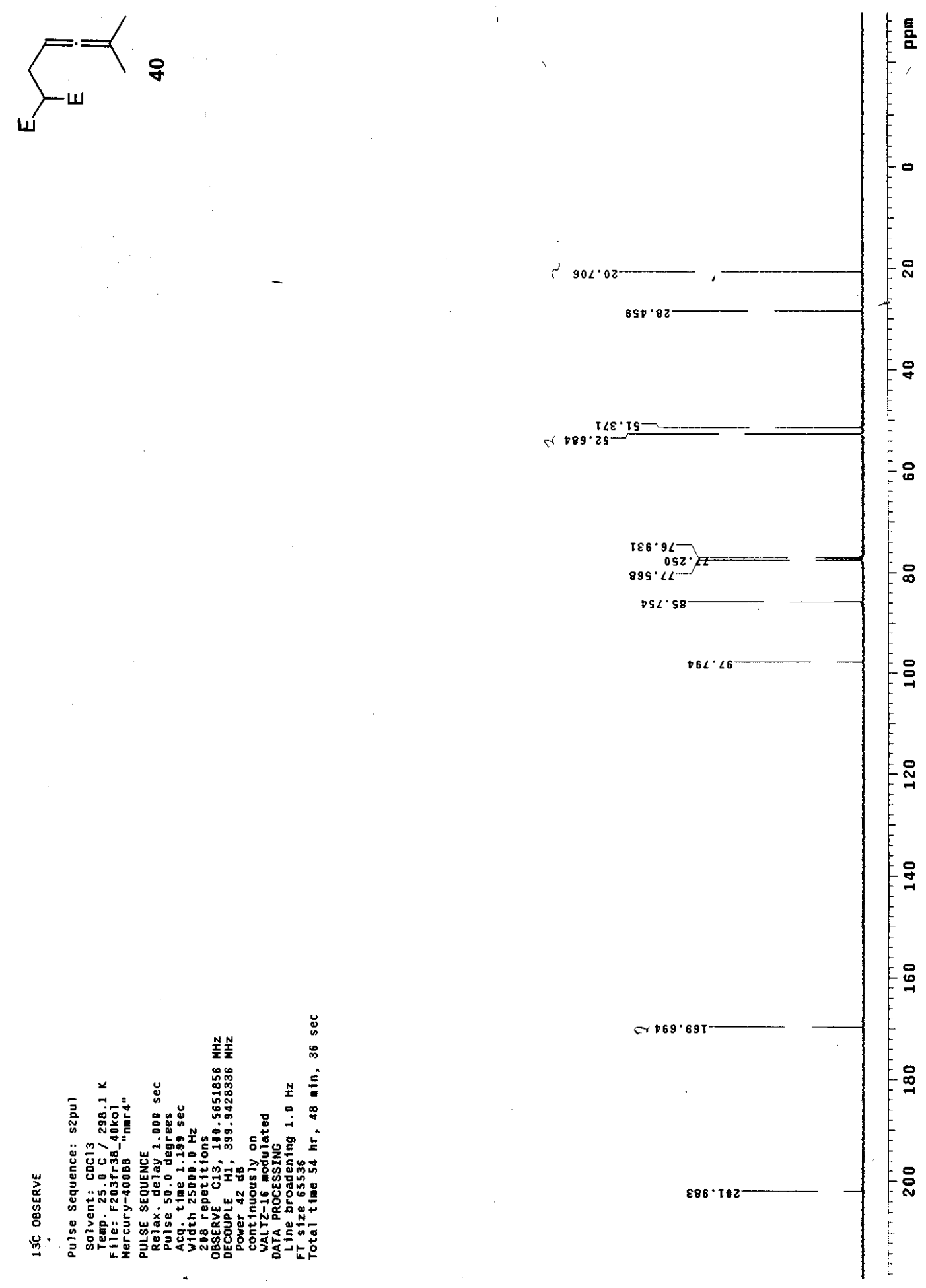